\title{
Stationary Properties of Driven Granular Gases
}

\author{
Dissertation \\ zur Erlangung des Doktorgrades \\ der Mathematisch-Naturwissenschaftlichen Fakultäten \\ der Georg-August-Universität zu Göttingen
}

vorgelegt von

Olaf Herbst

aus Hamburg

Göttingen, 2005 
D 7

Referentin: Prof. Dr. Annette Zippelius

Korreferent: Prof. Dr. Stephan Herminghaus

Tag der mündlichen Prüfung: 24. Februar 2005 


\section{Abstract}

We study driven granular gases in two dimensions. First we present systems of smooth particles between two vibrating walls. For these intrinsically inhomogeneous systems we measure all hydrodynamic fields for a wide range of inelasticities and system sizes using Event-Driven Molecular Dynamics simulations. Relating the locally measured pressure to the local density and temperature, we construct a constitutive relation. This relation can be considered as a local equation of state up to moderate inelasticities while it depends on the global system parameters for higher inelasticities. We also test the generalization of Fourier's law of thermal conductance, which relates the local heat flux to the local density and temperature gradients. We find reasonable agreement for moderately inelastic systems that are not too inhomogeneous. Furthermore, we present local velocity distributions of these systems and find that they do not scale. In particular, the distribution of the velocity component perpendicular to the walls differs from the one parallel to the walls. The Maxwell-Boltzmann distribution is found to be only a rough estimate as we find overpopulated high velocity tails that also depend on the position in the sample. Even a signature of the driving mechanism can be found in those tails. Finally we turn to homogeneously driven systems of rough particles with Coulomb friction. For these systems we analytically calculate the full dynamic evolution and stationary state values of the translational and rotational granular temperatures in mean field theory and find good agreement with Event-Driven Molecular Dynamics simulations. 



\section{Zusammenfassung}

In der vorliegenden Dissertation geht es um getriebene granulare Gase in zwei Dimensionen. Zunächst werden Systeme glatter Kugeln zwischen zwei vibrierenden Wänden betrachtet. Solche Systeme sind immer inhomogen. Mit Hilfe ereignisgesteuerter MolekulardynamikSimulationen messen wir alle hydrodynamischen Felder für einen großen Bereich von Systemparametern. Durch Verknüpfen des lokal gemessenen Drucks mit der lokalen Dichte und Temperatur erhalten wir eine konstitutive Gleichung. Diese kann für Systeme, die nicht allzu inelastisch sind, als Zustandgleichung aufgefasst werden, während sie für stärker inelastische Systeme von den globalen Systemparametern abhängt. Außerdem überprüfen wir eine Verallgemeinerung des Fourier'schen Wärmeleitungsgesetzes, das den lokalen Wärmestrom als Funktion der lokalen Dichte- und Temperaturgradienten ausdrückt. Dies funktioniert gut für mittlere Inelastizitäten, solange die Systeme nicht allzu inhomogen sind. Außerdem zeigen wir, dass die räumlich aufgelöste Geschwindigkeitsverteilung der Teilchen nicht durch ein Skalengesetz beschrieben werden kann, sondern dass das Verhalten nicht-universell ist. Insbesondere unterscheidet sich die Verteilungsfunktion für die Geschwindigkeitskomponente senkrecht zur Wand von der für die Komponente parallel zur Wand, und hohe Geschwindigkeiten werden viel häufiger beobachtet, als es eine MaxwellBoltzmann-Verteilung voraussagen würde. Zudem finden sich charakteristische Merkmale des Treibens in der Wahrscheinlichkeitsverteilung für hohe Geschwindigkeiten in der Mitte des Systems wieder. Zum Schluss werden homogen getriebene Systeme rauer Kugeln mit Coulomb'scher Reibung präsentiert. Für diese Systeme führen wir eine analytische Berechnung der zeitlichen Entwicklung der granularen Translations- und Rotationstemperaturen in Molekularfeldnäherung durch und beobachten eine gute Übereinstimmung mit Simulationen. 


\section{Contents}

Abstract iii

Zusammenfassung v v v v

$\begin{array}{ll}\text { 1. Introduction } & 1\end{array}$

2. Modelling Granular Gases $\quad 7$

2.1. Binary Collisions . . . . . . . . . . . . . . . . . . . . 9

2.1.1. Collisions with Incomplete Normal and Tangential Restitution _ . . . . 9

2.1.2. The Walton Model for Rough Particles with Coulomb Friction . . . . . 10

2.1.3. Smooth Particles . . . . . . . . . . . . . . . . . . . . . . 11

2.2. Homogeneous Bulk Driving . . . . . . . . . . . . . . . . 12

2.3. Boundary Induced Driving $\ldots \ldots \ldots$

3. Dimensional Analysis and Energy Balance $\mathbf{1 5}$

3.1. Vibrated Systems of Smooth Particles _ . . . . . . . . . . . . . . . 16

3.2. Homogeneously Driven Systems of Rough Particles with Coulomb Friction . 19

4. Definition of Microscopic and Coarse-Grained Observables 21

4.1. Summary: Granular Hydrodynamics ． . . . . . . . . . . . . . . . . . . 21

4.2. Microscopic Fields . . . . . . . . . . . . . . . . 21

4.3. Coarse-Graining the Fields . . . . . . . . . . . . . . . . . . 23

4.3.1. Densities of Mass, Momentum, and Energy, Velocity, and Temperature 23

4.3.2. Stress Tensor . . . . . . . . . . . . . . . . . . . . 24

4.3.3. Heat Flux and Energy Sink . . . . . . . . . . . . . . . 26

5. Measuring Observables in Simulations $\quad 29$

5.1. Event Driven Simulations of Vibrated Systems . . . . . . . . . . . . . . . . 29

5.2. Details of the Measurement of the Kinetic and Collisional Contributions . . . . 30

5.2.1. In the Bulk . . . . . . . . . . . . . . . . . . . 30

5.2.2. At the Wall . . . . . . . . . . . . . . . . . . . 33 
6. Hydrodynamic Fields in Simulations of Vibrated Systems 37

6.1. How to Scale the Driving as a Function of the Coefficient of Restitution . . . . 37

6.2. Density and Temperature Profiles . . . . . . . . . . . . . . . . . 39

6.3. Stress Tensor and Pressure . . . . . . . . . . . . . . . . . . . 41

6.4. Heat Flux . . . . . . . . . . . . . . . . . . . . . . . . 43

6.5. Local Energy Loss . . . . . . . . . . . . . . . . . . . . . . . 44

6.6. Gradients of the Density and Temperature . . . . . . . . . . . . . . . 44

6.7. Variation of the Coefficient of Restitution $\alpha$ at Fixed System Sizes . . . . . . . 45

6.8. Variation of the Density for Fixed Coefficients of Restitution . . . . . . . . 52

6.9. Summary of Scaling Behavior _. . . . . . . . . . . . . . . 57

6.9.1. Quasi-Elastic Limit . . . . . . . . . . . . . . . . . . . . 57

6.9.2. Low Density Limit . . . . . . . . . . . . . . . . . . . . . . . . . 59

7. Local Equation of State in Vibrated Systems 61

7.1. Deriving a Constitutive Equation . . . . . . . . . . . . . . . 62

7.2. Universality of the Constitutive Equation for Low to Moderate Inelasticities . 63

7.3. Non-Universality of the Constitutive Equation for Higher Inelasticities . . . . . 64

8. Fourier's Law and its Generalization for Vibrated Systems 67

8.1. Comparison of Heat Flux and Energy Loss to the Literature . . . . . . . . . . 67

8.2. Transport Coefficients From Simulations . . . . . . . . . . . . . . . . 70

8.2.1. The Fitting Procedure . . . . . . . . . . . . . . . 70

8.2.2. Discussion of Transport Coefficients . . . . . . . . . . . . . 71

9. Absence of Scaling for Velocity Distributions in Vibrated Systems $\quad \mathbf{7 5}$

9.1. Effects of the Discontinuity at a Driving Wall . . . . . . . . . . . . 76

9.2. Non-Universal High-Velocity Tails . . . . . . . . . . . . . . . . . . . 78

10. Homogeneously Driven Systems of Spheres with Coulomb Friction $\quad 83$

10.1. Impact-Angle Probability Distribution . . . . . . . . . . . . . . . . 84

10.2. Differential Equations for the Time Evolution of Temperatures . . . . . . . . 85

10.2.1. Model A: Constant Coefficient of Tangential Restitution $\beta=\beta_{0} \ldots$. . 86

10.2.2. Model B: Averaged Simplified Coulomb Friction $\rightarrow \beta=\left\langle\beta\left(\gamma_{12}\right)\right\rangle_{\gamma_{12}} .87$

10.2.3. Model C: Averaged Real Coulomb Friction $\rightarrow \beta=\langle\beta(\gamma)\rangle_{\gamma}(R) \ldots$. . 87

10.2.4. Model D: Simplified Coulomb Friction $\rightarrow \beta=\beta\left(\gamma_{12}\right) \ldots \ldots$. . . . 89

10.2.5. Model E: Real Coulomb Friction $\rightarrow \beta=\beta(\gamma) \ldots \ldots$. . . . . . . 91

10.3. Steady State . . . . . . . . . . . . . . . . . . . . 92

10.3.1. Analytical Results . . . . . . . . . . . . . . . . . . . 92

10.3.2. Comparison with Simulations _ . . . . . . . . . . . . 96

10.4. Approach to the Steady State ～. . . . . . . . . . . . . . . . 100

10.4.1. Close to the Steady State . . . . . . . . . . . . . . . . . . 100

10.4.2. Full Dynamic Evolution . . . . . . . . . . . . . . . . . . . 101 
10.5. Discussion . . . . . . . . . . . . . . . . . 101

$\begin{array}{ll}\text { 11.Summary and Outlook } & 105\end{array}$

$\begin{array}{lr}\text { A. Heat Flux for Non-Zero Velocity Fields } & 107\end{array}$

B. Simple Energy-Balance Argument for Two Distinctively Driven Walls 111

C. Time Evolution of Energies Using a Pseudo-Liouville Operator Approach113

C.1. Time Evolution in Terms of the Pseudo-Liouville Operator . . . . . . . . . 113

C.2. Particle-Particle Contribution $\left\langle i \mathcal{L}_{+}^{\mathrm{pp}} E_{\mathrm{x} / \mathrm{y} / \mathrm{tr} / \mathrm{rot}}\right\rangle_{t}$ to $\frac{d}{d t} T_{\mathrm{x} / \mathrm{y} / \mathrm{tr} / \mathrm{rot}}(t) \ldots \ldots 116$

C.2.1. PP Contribution $\left\langle i \mathcal{L}_{+}^{\mathrm{pp}} E_{\mathrm{x} / \mathrm{y}}\right\rangle_{t}$ to the Change of Translational Energy . . 118

C.2.2. PP Contribution $\left\langle i \mathcal{L}_{+}^{\mathrm{pp}} E_{\mathrm{rot}}\right\rangle_{t}$ to the Change of Rotational Energy . . . 127

C.2.3. For Comparison: PP Contributions for 3D Systems . . . . . . . . . 130

C.3. Particle-Wall Contribution $\left\langle i \mathcal{L}_{+}^{\mathrm{pw}} E_{\mathrm{x} / \mathrm{y} / \mathrm{tr} / \mathrm{rot}}\right\rangle_{t}$ to $\frac{d}{d t} T_{\mathrm{x} / \mathrm{y} / \mathrm{tr} / \mathrm{rot}}(t) \ldots \ldots 130$

C.3.1. PW Contribution $\left\langle i \mathcal{L}_{+}^{\mathrm{pw}} E_{\mathrm{x} / \mathrm{y}}\right\rangle_{t}$ for Smooth Particle-Wall Interactions . . 132

C.4. Total Time Derivative of Partial Temperatures $\frac{d}{d t} T_{\mathrm{x} / \mathrm{y}}(t)$ for Smooth Particles . 133

C.5. Energy Balance for Smooth Particles in the Stationary State . . . . . . . . . 134

C.6. Why and How Do the Simple and the Refined Energy Balance Differ? . . . . . 135

C.7. Artifact of the Walton Model: Surviving Rotational Energy without Driving . . 136

$\begin{array}{lr}\text { Bibliography } & 139\end{array}$

$\begin{array}{ll}\text { Acknowledgements } & 151\end{array}$

$\begin{array}{ll}\text { List of Publications and Meetings } & 153\end{array}$

$\begin{array}{lr}\text { Curriculum Vitae } & 155\end{array}$ 



\section{Introduction}

Granular materials have attracted much scientific attention in the last two decades. This is not surprising since granular materials are ubiquitous. For example, more than half the world's raw materials are found in a granular state leading to a large industrial interest. Industrial aims are, to mention a few, improving granular flow through pipes, better compacting ground coffee or building longer lasting roads, whose beds consist of gravel, i.e. granular materials. Granular matter is being intensively studied in two major phases ${ }^{1}$ : the "gas" phase, in which the grains are fluidized and interact by near-instantaneous collisions, and the static and quasi-static phases, of which sand-piles constitute a typical example. Intermediate (liquid-like) phases are considered as well. None of these regimes is close to being fully understood. In this thesis we will focus on the gas phase of granular materials.

The standard paradigm of a granular gas is a collection of hard spheres whose inelastic collisions are characterized by a constant coefficient of normal restitution, i.e. in a collision the particles lose a fraction of their relative velocity. This model can be extended to rough particles with Coulomb friction [WB86]. Essentially all difficulties and many of the interesting features pertaining to granular gases can be traced to the fact that macroscopic grains experience dissipative interactions on the grain scale. This distinguishes them from molecular gases. Most states of granular matter are meta-stable (e.g., the ground state of a sand-pile is one in which all grains rest on the floor), and energy is not conserved (i.e., it "disappears" into the internal degrees of freedom). Therefore a continuous energy injection is vital in order to maintain non-static steady states. Without forcing, a gas of inelastic spheres would collapse, even in the absence of gravity. Thus granular gases are inherently of non-equilibrium nature, and many well internalized notions have to be revisited. In particular, the applicability of hydrodynamics [GS95, GZBN97, BDKS98, SG98, Gol03] is still an object of debate [DLK95, TG98, Kad99, Gol00, Tos04] - despite the similarities between hydrodynamics of elastic hard-sphere systems and granular hydrodynamics, concerning e.g. the appearance of instabilities [JNB96, LMS02a, LMS02b, MPSS04].

The non-trivial nature of these stationary states has been elucidated in experiments of vibrating grains. In this context the field witnessed the use of increasingly sophisticated measurement methods ranging from ultra fast photography, to MRI and gamma-ray tracking methods [Gol03]. These methods also allow the study of dynamic properties, e.g., the internal structure

\footnotetext{
${ }^{1}$ the term "phase" is not meant in the thermodynamic sense here
} 
of a shock [RBSS02, BMSS02], since typical mean free paths in granular media can be of the order of centimeters or larger. Some of the most striking features found in experiments of the stationary state are the oscillon excitation [UMS96, UMS98, US00], non-Gaussian velocity distributions [OU98, UO01, LCG99, RM00] and cluster formation [KWG97, FFL99, FWE ${ }^{+}$99]. Furthermore, numerous known hydrodynamic instabilities have been shown to have granular counterparts [Gol03, Sun03, God03].

Among the major achievements on the theoretical side are the development of hydrodynamic descriptions [SG98, BDKS98, MGSB99] and ring kinetic theory [vNEB98] of frictionless (i.e. smooth) granular gases, the discovery of the mechanism of clustering in granular gases [GZ93, GTZ93], and some of its interesting consequences (such as the Maxwell demon effect [vdWvdMVL01]), and the discovery of the source of the normal stress differences as a Burnett effect [SG98, BDKS98, SGN96, GS96]. Other systems, such as needles [AGZ98, HAZ99, VT04], have been studied as well. Although not of direct experimental relevance, the homogeneous cooling state has been studied in great detail, see e.g. Refs. [GTZ93, LHMZ98, HOB00, HHZ00] and references therein.

Kinetic theory is one very successful theoretical approach to studying granular systems at fluid densities, see e.g. Refs. [vNEB98, BDS99, Duf01, Gol03] and references therein. Particular applications to driven granular gases can be found in Refs. [BC98, vNE98, CLH00, KBN01, BTF01]. Much of this work is based on the Boltzmann or Boltzmann-Enskog equation, modified for inelastic collisions. One method to solve this nonlinear equation is based on the local equilibrium distribution [JS83], which is only known for the elastic case. For systems with strongly inelastic collisions, the stationary state is unknown so that a systematic discussion of transport properties within kinetic theory is severely hampered.

Granular hydrodynamics is the other major approach. Hydrodynamic studies [GZBN97, BRMM00, LMS02b, MPSS04] have been motivated partly by the search for an understanding of temperature and density profiles [GZBN97], but also by experiments on hydrodynamic-like instabilities [JNB96]. The standard way of solving the hydrodynamic field equations requires expressing the pressure in terms of the density and temperature, usually by an equation of state. Using the global quantities several proposals for an equation of state have been made, either interpolating between the high and low density limit [GZBN97, Lud01] or invoking the Boltzmann-Enskog equation [BT02b] for inelastically colliding particles. It is not clear whether an equation of state also holds for the local hydrodynamic fields in a strongly driven, non-equilibrium system, where the fields are very inhomogeneous. There have also been several experimental investigations [WHJ95, EFL00] attempting to extract either an equation of state or more generally a scaling relation for the corresponding variables. To close the set of hydrodynamic equations a second constitutive relation is needed. In classical hydrodynamics this relation is usually given by Fourier's law of thermal conductance. For granular media it is known that, in general, the heat flux does not obey this law [JR85, SG98]. In addition to the usual coefficient of thermal conductivity, relating the heat flux to the temperature gradient, 
a new transport coefficient is necessary that expresses a contribution of the density gradient. Measuring all these hydrodynamic fields, testing several proposed equations of state as well as the generalized Fourier law and extracting transport coefficients for the heat flux from computer simulations for a wide range of parameters is a major focus of this thesis.

Computer simulations are a key tool in studying granular systems. Their importance lies in their ability to track the trajectory of every single particle, a task that would be beyond available experimental techniques, especially in three dimensions. Most simulations of vibrated granular gases have been based either on the very efficient event-driven (ED) molecular dynamics algorithm, cf. Refs. [Rap88, Lub91, LHB94, MB97, LM98, BT02b] and references therein, or the direct-simulation Monte Carlo method (DSMC) [Bir94, BC98, BRMM00, PLMV99, BMPV01] and its generalization including collisional transfer called Enskog-Simulation Monte Carlo (ESMC) [MS96, BDS97]. While DSMC (or ESMC) is a powerful tool for solving the Boltzmann (or Boltzmann-Enskog) equation numerically, one of the disadvantages of DSMC compared to ED is that DSMC does not allow for clustering on the grain scale. ED simulations, as used in this thesis, very efficiently numerically integrate Newton's equations of motion while only assuming the time of collisional contact to be small compared to the free flight time.

The driving mechanism is a crucial point of any model describing driven granular fluids. Easy to describe theoretically are homogeneous driving mechanisms. One approach utilizes stochastic bulk heating by uncorrelated random forces, which act on every particle at every instant of time [WM96, vNE98, vNETP99, $\mathrm{BBR}^{+}$02, $\mathrm{HCZ}^{+}$05]. In Ref. [BTF01] random coefficients of restitution were considered with a probability distribution allowing for values both smaller and greater than one. However, this yields non-universal properties depending on the specific form of this distribution. The multiplicative bulk driving defined in terms of stochastic collision rules in Ref. [CLH00] is similar in spirit. Compared to homogeneous bulk driving, a driving mechanism which acts only at the boundary of the system is much closer to experiments. In Ref. [GZBN97] the energy influx at the boundary has been modeled by a heuristically motivated ansatz for the heat current, while Refs. [BC98, RC02] assume heating at the boundary through thermal walls. Closest to experiments but hardest to analyze are vibrating walls [MB97, BRMM00, BT02b, HMOZ04]. While homogeneously driven rough spheres will be considered as well, most of this thesis deals with systems of smooth particles driven by vibrating walls in an idealization that has been used before in, e.g., Refs. [BRMM00, BT02b], and with slight modifications in Refs. [MB97, DP98, Kum98, Kum99b].

If the system is driven through the boundaries, inhomogeneous density and temperature profiles are measured [BT02b, HMOZ04]. For low densities the computed temperature profiles agree well with hydrodynamic theory [GZBN97, BRMM00]. For moderate or high densities the profiles are not well understood with the exception of almost elastically colliding particles. To our knowledge the full stress tensor, including potential contributions, has only been computed for freely cooling systems [LS00]. In simulations of driven granular gases [BC98] the collisional part of the stress tensor has not been measured. In Ref. [BT02b] the stress tensor 
is not measured directly but instead computed from an assumed local equation of state. Similarly, there is a collisional contribution to the heat flux (in addition to the kinetic part) that - to our knowledge - has been neglected in all previous simulations found in the literature. In the simulations presented here both components of the stress tensor and the heat flux are measured directly.

Inspired by experiment [RM00], a lot of emphasis has been put on the tails of the velocity distribution functions, which were found to be overpopulated as compared to a Maxwellian. In fact, all intermediate types of decay between a Gaussian and an exponential have been observed [PLMV99, BT02b, vZM04, BRM03]. In addition, mixtures [BT02b, BT02a, PCMP03] and rough spheres [Lud95] have been investigated as well as hydrodynamic instabilities such as convection [ $\mathrm{LCB}^{+} 94, \mathrm{RRC00}$ ] and pattern formation [LCRD96]. The velocity distributions shown here are of very high accuracy. In particular, the tails have been measured for a range of up to forty standard deviations in the velocity which is about ten times of what can be found in the literature. This reveals surprising new details of the velocity distributions not seen before, e.g. the signature of the particular driving at the boundary can be discovered in the tails of the velocity distribution in the middle of the system.

In this thesis we present event-driven simulations of inelastic smooth spheres in two dimensions confined between two vibrating walls without gravity. Our focus is on the stationary state, which is reached when dissipation by particle collision equals energy injection due to the vibrating walls. We show stationary state profiles of the density, the granular temperatures, the full stress tensor (including collisional contributions), and the full heat flux (again, including collisional contributions). These hydrodynamic fields are shown to be strongly inhomogeneous due to the driving walls - even in the range of parameters, where clustering is only a minor effect. This has led us to:

(a) derive a constitutive equation by relating the measured hydrodynamic fields, granular temperature $T(x)$, area fraction $\phi(x)$ and pressure $p(x)$, at each point $x$. We then check whether this constitutive equation is universal or whether it depends on global system parameters of the model, like the aspect ratio of the cell, the overall area fraction or the coefficient of restitution of the disks. For moderately inelastic systems $(\alpha=0.9)$ the constitutive equation is (almost) independent of the remaining global system parameters so that the constitutive equation can be interpreted as a local equation of state for a driven granular gas in the stationary state, even though the latter is highly inhomogeneous with heterogeneous temperature and density profiles. In contrast, for strongly inelastic systems $(\alpha=0.5)$ the constitutive equation depends significantly on the global system parameters so that the concept of a local equation of state cannot be sustained in this case.

(b) check Fourier's law of thermal conductance and its generalization for granular materials which connects the heat flux to the gradients of the temperature and density profiles. Additionally, we extract the transport coefficients connected to the heat flux, i.e. the heat conductivity 
$\kappa$ and the "new" transport coefficient $\mu$. This requires extremely precise measurements of the hydrodynamic fields, especially of the density and temperature profiles in order to limit the fluctuations of their gradients. The generalized Fourier law is a very good approximation as long as the systems are not too inelastic and as long as the gradients do not become too large as expected for a gradient expansion. Consequently the best agreement is found for moderately dense and quasi-elastic to moderately inelastic systems. We also compare the measured heat flux to the theoretical prediction of Jenkins and Richman [JR85] and find that the theory is very good for quasi-elastic systems (coefficient of restitution $\alpha \geq 0.99$ ). Finally, we quantify the improvement obtained when fitting the measured heat flux to the generalized Fourier law instead of to the plain Fourier law.

We furthermore discuss the one-particle distribution function in the stationary state and show that:

(c) the local distribution $f_{x}\left(x, v_{x}\right)$ of $v_{x}$, the velocity in the direction of driving, is not a function of the rescaled variable $v_{x} / \sqrt{T_{x}(x)}$ alone. Similarly, curves of $f_{y}\left(x, v_{y}\right)$ cannot be mapped onto a master curve for different $x$, when plotted against $v_{y} / \sqrt{T_{y}(x)}$. Here $T_{i}(x)$ denotes the local granular temperature associated with the translational motion in the $i$-direction. We find deviations from scaling at small and large arguments.

(d) the local velocity distributions $f_{x}$ and $f_{y}$ have high-velocity tails whose decay ranges from stretched exponential to almost Gaussian. For this purpose we have measured the velocity distribution of up to forty standard deviations on the velocity axis. The particular type of decay depends on the position in the sample, the overall particle density and the coefficient of restitution. Furthermore, the decay of $f_{x}$ for large velocities is generally different from that of $f_{y}$.

All these results have been obtained for vibrated systems of smooth particles, i.e. we have ignored sliding friction and tangential restitution. While theories for smooth particles capture a vast amount of phenomena of granular materials, at least qualitatively, it is known that friction does play an important role [ $\left.\mathrm{vZKG}^{+} 04\right]$. Unfortunately, it is severely more complicated to analytically analyze systems of frictional particles, because the collision rules for Coulomb friction are far more complex. In this thesis we also investigate systems of frictional particles. To keep the analysis simple, we restrict ourselves to the homogeneously driven systems and a parameter range where clustering does not play an important role. In this regime all hydrodynamic fields are very homogeneous. We will therefore focus on the dynamic evolution and the stationary state values of the mean translational and rotational temperatures. Results of an analytic mean field theory using a Pseudo-Liouville-Operator ansatz agree well with event-driven simulations. Because the calculation is quite elaborate when the full Walton model [WB86] is used, even for this rather simple question of stationary state temperatures, we calculate effective coefficients of tangential restitution in various levels of approximation. When comparing the analytical results using these effective coefficients to simulations of particles with Coulomb 
friction, we find that the simplest approximation already yields qualitative agreement. Only the full Walton model, though, is able to predict the correct behavior for the full range of parameters. We conclude that the simplest approximation introduced in this thesis may be a good starting point for future investigations of frictional particles but we must admit that realistic Coulomb friction is a subtle problem.

The outline of this thesis is as follows: In chapter 2 we introduce the model and specify the driving mechanisms. In chapter 3 we non-dimensionalize the system and briefly discuss the balance of injected and dissipated energy. In chapter 4 we define the hydrodynamic fields used for vibrated systems. In chapter 5 we briefly introduce the reader to the Event Driven simulation algorithm and discuss some details of how to measure hydrodynamic fields in an ED simulation. In chapter 6 we present data from our simulations for the profiles of the density, the temperature, the components of the stress tensor, the heat flux, and the local energy loss. In chapter 7 we relate the local density, pressure and temperature to "experimentally" derive an equation of state and check its universality. In chapter 8 we relate the local heat flux to the local gradients of the temperature and density, test the validity of the generalized Fourier law and evaluate the corresponding transport coefficients. In chapter 9 we discuss velocity distribution functions and their scaling behavior. Finally we turn to homogeneously driven systems of rough particles with Coulomb friction in chapter 10, where we focus on the granular temperatures and discuss various levels of approximations to the full collision rules and their shortcomings. In chapter 11 we will give a short summary and an outlook. 


\section{Modelling Granular Gases}

Granular materials are defined as materials whose elementary constituents are macroscopic solid particles. The particles being macroscopic implies that they interact dissipatively. These are the two defining properties of a granular medium. The energy dissipated in the collisions will be transformed to internal energy, i.e. it will eventually be transformed to heat. This leads to an increase of the thermodynamic temperature of the particles. For a single collision this increase can often be neglected. It is therefore legitimate to assume the additional internal energy, that is produced in collisions, to be radiated off (in vacuum) or absorbed and carried away quickly by a surrounding background medium, e.g. air. Because granular materials are of importance mainly around room temperature (or below), thermodynamic fluctuations ( $k_{B} T \approx$ $4 \times 10^{-21} \mathrm{~J}$ ) are negligible compared to the kinetic energy of a granular particle which is typically much greater than $10^{-16} \mathrm{~J}^{2}$. Here, $k_{B}$ denotes Boltzmann's constant. For the rings of Saturn, which are another important granular gas, this separation of scales is even larger. These arguments have led to a description of granular materials by models that do not conserve energy but only momentum. From now on thermodynamic temperatures will no longer be considered and all further references to temperatures will refer to granular temperatures, i.e. the square of the fluctuations around the mean velocity of the particles. Furthermore we will ignore interactions of the particles with a surrounding medium, such as air, and thus assume the particles to be in a vacuum environment.

We investigate driven granular gases in two dimensions consisting of $N$ identical inelastic hard particles of diameter $a$, mass $m$, and moment of inertia $I$. These are confined to a rectangular box with edges of length $L_{x}$ and $L_{y}$ in a vacuum zero-gravity environment. We consider both disks and spheres. Two-dimensional particles have three degrees of freedom, two translational ones and one rotational. For smooth particles this reduces to two (translational) degrees of freedom. The inelastic nature of the collisions is often modelled by a constant coefficient of normal restitution $\alpha$, i.e. in a collision the particles lose a fraction of their relative velocity. When realistic particles collide, they also experience friction reducing their relative rotational

\footnotetext{
${ }^{1}$ At room temperature the specific heat of most (condensed) materials is of the order $10^{3} \mathrm{~J} /[\mathrm{kg} \mathrm{K}]$ (e.g. steel (iron) $\sim 0.5 \times 10^{3} \mathrm{~J} /[\mathrm{kg} \mathrm{K}]$, common minerals $\left.\sim 1.3 \times 10^{3} \mathrm{~J} /[\mathrm{kg} \mathrm{K}]\right)$. The energy dissipated in a collision is usually less than $20 \%$ of the kinetic energy of the particle and thus leads to an increase of the particle's (thermodynamic) temperature $\Delta T<10^{-4} v^{2} \mathrm{~K}$ (where $v$ is the dimensionless velocity in units of $\mathrm{m} / \mathrm{s}$ ). For typical collisions of relative velocity $\lesssim 10 \mathrm{~m} / \mathrm{s}$ this is negligible $\left(\Delta T=10^{-2} \mathrm{~K} \ll T_{\text {room }} \approx 300 \mathrm{~K}\right)$.

${ }^{2}$ Kinetic energy of steel (iron) beads of $1 \mathrm{~mm}$ diameter (density $7.87 \mathrm{~g} / \mathrm{cm}^{3}$ ) and velocity $1 \mathrm{~m} / \mathrm{s} \mathrm{is} \sim 10^{-6} \mathrm{~J}$. Equivalently, $k_{B} T$ corresponds to a velocity (change) of $\sim 10^{-7} \mathrm{~m} / \mathrm{s}$.
} 
motion. As will be discussed below, this can be described by two more dimensionless material constants, the coefficient of Coulomb friction $\mu$ (for sliding) and the coefficient of tangential restitution $\beta_{0}$ for sticking contacts.

The canonical 2-dimensional spherical particle is a disk. If a system of spheres is considered we simply assume that three-dimensional particles are confined to motion in 2 dimensions. This can be accomplished by perfectly smooth walls that do not interact with the spheres (except for confining their motion to two dimensions by elastically and smoothly repelling the spheres), even though the particles themselves may not be perfectly smooth. If the particles are considered to be smooth to begin with, there is no difference between disks and spheres. For rough particles the only difference between disks and spheres lies in their moment-of-inertia-to-mass ratio $q:=4 I /\left[m a^{2}\right]$ being $1 / 2$ instead of $2 / 5$.

To maintain a non-trivial stationary state energy must be injected continuously. Driving a granular material can be realized through the boundaries, i.e. a local heating [HHL98, LSM99, SMR99, SM01] or homogeneously, e.g. by a random energy source in different variations [PLMV98, BSS99, vNE98, vNETP99, CLH00, CLH02]. We will use boundary induced as well as homogeneous bulk driving.

Driving through the boundaries is accomplished by vibrating the walls perpendicular to the $x$ direction in an idealized saw-tooth manner (see below), while periodic boundary conditions are imposed in the $y$-direction. Fig. 2.3 on page 13 shows a typical snapshot of a vibrated system. Clearly, the system is not homogeneous but shows an increased density in the middle of the system.

Homogeneous driving has no close experimental equivalents but is easier to analyze. At a fixed frequency every particle gets a kick of random strength and direction drawn from a Gaussian distribution of fixed driving temperature. Details will be given below. Experimental setups that may be approximated by this model are an air table or a mono-layer of particles on a vibrating plane under gravity [OU98]. In both cases the particles stay very close to the underlying plane and can only move parallel to it. When we use a two-dimensional description of this system the driving can be considered random, indeed. Even homogeneously driven systems experience spatial variations of the density, for example, if the system is large enough and/or sufficiently inelastic compared to the driving. In that case clustering can occur like in the freely cooling case [GZ93] and/or the formation of patterns can be observed [OU98, OU99].

In the simple model used here the system evolves in time solely through ballistic center-ofmass motion, binary inelastic collisions and, depending on the driving mechanism, particlewall collisions or homogeneous driving. 


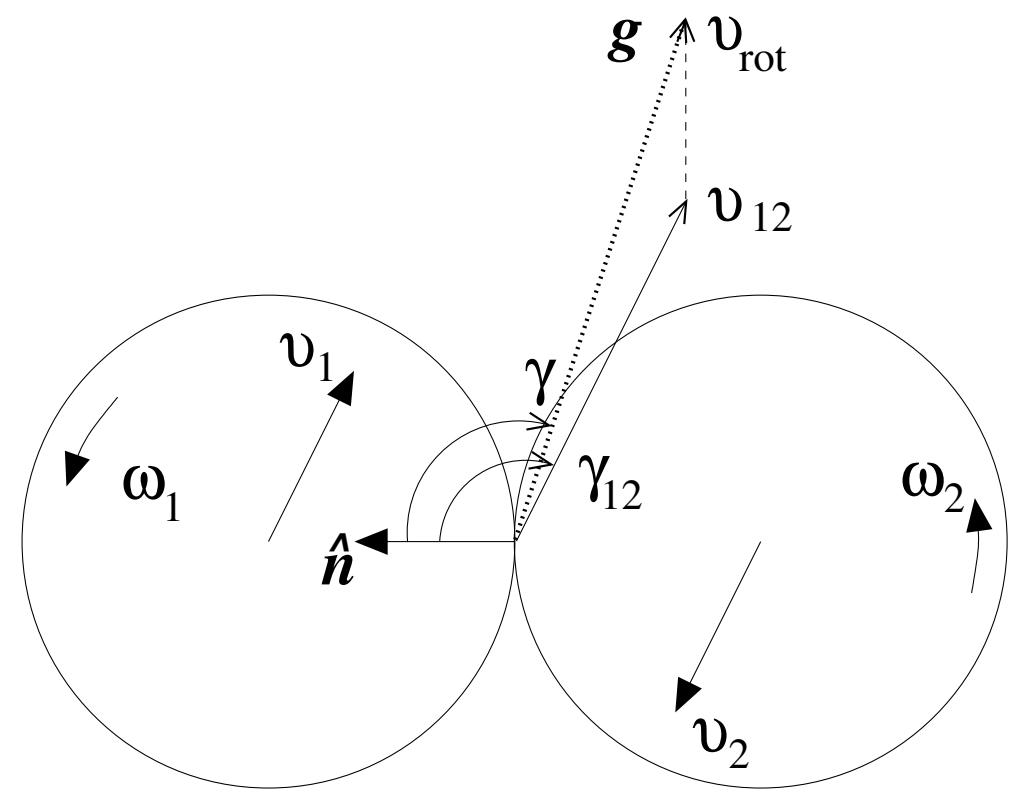

Figure 2.1.: Sketch of 2 colliding particles in the center-of-mass reference frame. Shown are the relative velocity of the contact points $\boldsymbol{g}$, the impact angle $\gamma$, and the angle $\gamma_{12}$ between the relative translational velocity of the particles and their contact normal.

\subsection{Binary Collisions}

The inelastic nature of inter-particle collisions is the most important characteristic of granular media. We will use the Walton Model [WB86] - introduced below - to describe collisions of inelastic frictional particles. The Walton model can be formulated in terms of coefficients of incomplete normal and tangential restitution [FLCA94, HHZ00] and is thus a generalization of the collsion rules for rough particles with constant coefficients of restitution [HZ97]. The special case of smooth particles is contained in this description.

\subsubsection{Collisions with Incomplete Normal and Tangential Restitution}

Before we come to the details of the Walton model, we briefly recall the binary collision rules for collisons with incomplete normal and tangential restitution [HZ97, WB86, FLCA94, HHZ00].

Consider two colliding particles. Their center-of-mass velocities and angular velocities before a collision shall be denoted by $\boldsymbol{v}_{1}, \boldsymbol{v}_{2}, \boldsymbol{\omega}_{1}$ and $\boldsymbol{\omega}_{2}$. Post-collisional quantities are primed. The relative velocity of the contact point is given by

$$
\boldsymbol{g}=\boldsymbol{v}_{1}-\boldsymbol{v}_{2}+\frac{a}{2} \hat{\boldsymbol{n}} \times\left(\boldsymbol{\omega}_{1}+\boldsymbol{\omega}_{2}\right)
$$


The relative velocity after collision is given by

$$
\begin{aligned}
\hat{\boldsymbol{n}} \cdot \boldsymbol{g}^{\prime} & =-\alpha(\boldsymbol{g}, \hat{\boldsymbol{n}})(\hat{\boldsymbol{n}} \cdot \boldsymbol{g}) \quad \text { with } \quad \alpha(\boldsymbol{g}, \hat{\boldsymbol{n}}) \in[0,1], \\
\hat{\boldsymbol{n}} \times \boldsymbol{g}^{\prime} & =-\beta(\boldsymbol{g}, \hat{\boldsymbol{n}})(\hat{\boldsymbol{n}} \times \boldsymbol{g}) \quad \text { with } \quad \beta(\boldsymbol{g}, \hat{\boldsymbol{n}}) \in[-1,1]
\end{aligned}
$$

where $\alpha(\boldsymbol{g}, \hat{\boldsymbol{n}})$ and $\beta(\boldsymbol{g}, \hat{\boldsymbol{n}})$ are the coefficients of restitution, which in general depend on $\boldsymbol{g}$ and $\hat{\boldsymbol{n}}$. We will later assume $\alpha$ to be constant and allow $\beta$ to depend on the angle $\gamma$ between $\boldsymbol{g}$ and $\hat{\boldsymbol{n}}$ in order to account for the different energy loss mechanisms of sliding and sticking contacts in the Walton model (cf. next section). The impact angle satisfies $\gamma \in\left[\frac{\pi}{2}, \pi\right]$, such that $\cos \gamma=\hat{\boldsymbol{n}} \cdot \boldsymbol{g} /|\boldsymbol{g}|<0$. The values $\alpha=1$ and $\beta=-1$ describe perfectly smooth and perfectly elastic particles, while $\alpha=1$ and $\beta=+1$ describe perfectly rough and perfectly elastic particles. These two cases conserve energy. If $\alpha<1$ and/or $|\beta|<1$ energy will not be conserved but decreased in each collision. Very often these coefficients $\alpha$ and $\beta$ are assumed to be constants.

The two constitutive equations (2.2) and (2.3) plus the conservation laws for linear and angular momenta determine the post-collisional velocities

$$
\begin{array}{cc}
\boldsymbol{v}_{1}^{\prime}=\boldsymbol{v}_{1}+\Delta \boldsymbol{v}_{\mathrm{pp}}, & \boldsymbol{v}_{2}^{\prime}=\boldsymbol{v}_{2}-\boldsymbol{\Delta} \boldsymbol{v}_{\mathrm{pp}} \\
\boldsymbol{\omega}_{1}^{\prime}=\boldsymbol{\omega}_{1}+\Delta \omega_{\mathrm{pp}}, & \boldsymbol{\omega}_{2}^{\prime}=\omega_{2}+\Delta \omega_{\mathrm{pp}}
\end{array}
$$

where

$$
\begin{aligned}
& \boldsymbol{\Delta} \boldsymbol{v}_{\mathrm{pp}}=-\frac{(1+\alpha)}{2}\left(\hat{\boldsymbol{n}} \cdot \boldsymbol{v}_{12}\right) \hat{\boldsymbol{n}}-\eta \hat{\boldsymbol{n}} \times\left(\boldsymbol{v}_{12} \times \hat{\boldsymbol{n}}+\frac{a}{2} \boldsymbol{\omega}_{12}\right) \\
& \boldsymbol{\Delta} \boldsymbol{\omega}_{\mathrm{pp}}=\frac{2 \eta}{q a}\left(\hat{\boldsymbol{n}} \times \boldsymbol{v}_{12}+\frac{a}{2} \hat{\boldsymbol{n}} \times\left(\hat{\boldsymbol{n}} \times \boldsymbol{\omega}_{12}\right)\right)
\end{aligned}
$$

with $\boldsymbol{v}_{12}=\boldsymbol{v}_{1}-\boldsymbol{v}_{2}, \boldsymbol{\omega}_{12}=\boldsymbol{\omega}_{1}+\boldsymbol{\omega}_{2}$,

$$
\eta \equiv \eta(\gamma) \equiv \eta(\beta(\gamma)):=\frac{q}{1+q} \frac{1+\beta(\gamma)}{2},
$$

such that $0 \leq \eta(\beta) \leq \frac{q}{q+1}<1$, and

$$
q:=\frac{4 I}{m a^{2}}
$$

( $q=0.4$ for homogeneous spheres, $q=0.5$ for homogeneous disks).

\subsubsection{The Walton Model for Rough Particles with Coulomb Friction}

Although the widely used collision rules based on constant coefficients of restitution faithfully reproduce many of the experimental findings [TV02, WHP01, HZ97, WP02], constant coefficients of restitution are known to correspond only approximately to reality [GZ99, RPBS99, BP01]. In particular, they cannot describe frictional dry sliding. A now commonly accepted 
model for frictional contacts is based on work by Walton [WB86]. For every collision it chooses the more appropriate of two types of dissipative interactions, either Coulomb friction (for sliding contacts) or incomplete tangential restitution (with a constant coefficient of tangential restitution - for sticking contacts). The criterion is based on the size of the angle between the relative velocity of the contact point and the line between the two centers of masses. Even though this model is very simple, it describes the experimental data surprisingly well [FLCA94]. Since the Walton model for Coulomb friction can be expressed in terms of an impact-dependent coefficient of tangential restitution [FLCA94, HHZ00], its implementation in simulations is straightforward, while theoretical analyses are significantly more complicated [HHZ00, Her00, HMOZ04].

The Walton model [WB86] contains three (constant) material parameters: the coefficient of normal restitution $\alpha$, the coefficient of Coulomb friction $\mu$ for sliding contacts, and the coefficient of tangential restitution $\beta_{0}$ for sticking contacts. As mentioned above this three-parameter model can be translated to a velocity dependent coefficient of tangential restitution

$$
\beta(\gamma)=\min \left[-1-\frac{1+q}{q}(1+\alpha) \mu \cot \gamma, \beta_{0}\right],
$$

that depends on all three material constants as well as the impact angle $\gamma$ in a collisions. For details see, e.g., Refs. [FLCA94, HHZ00, Her00]. Inserting Eq. (2.8) into Eq. (2.6) leads to $\eta \equiv \eta(\gamma)=\min \left[\frac{1+\alpha}{2} \mu, \eta_{0}\right]$. The critical angle $\gamma_{0}$ which marks the transition between sliding and sticking contacts is given by

$$
c:=-\cot \gamma_{0}=\frac{q}{1+q} \frac{1+\beta_{0}}{1+\alpha} \frac{1}{\mu}>0 .
$$

These equations are valid for 2 dimensions just as well as for 3 dimensions.

Fig. 2.2 shows how the coefficient of tangential restitution $\beta(\gamma)$ varies as a function of the impact angle $\gamma$ for different values of the coefficient of Coulomb friction $\mu$.

\subsubsection{Smooth Particles}

Even though the simulation is capable of dealing with rough particles, for the sake of analytical simplicity all simulations of boundary driven systems presented will be for smooth particles. In that case, $\boldsymbol{g}=\boldsymbol{v}_{12}, \mu=0$, and $\beta_{0}=-1$ such that Eqs. (2.5) simplify to [BSHP96]

$$
\begin{aligned}
& \boldsymbol{\Delta} \boldsymbol{v}_{\mathrm{pp}}=-\frac{(1+\alpha)}{2}\left(\hat{\boldsymbol{n}} \cdot \boldsymbol{v}_{12}\right) \hat{\boldsymbol{n}} \\
& \boldsymbol{\Delta} \boldsymbol{\omega}_{\mathrm{pp}}=0
\end{aligned}
$$

(and each particle conserves its rotation for all times). Therefore we only have to consider translational motion when dealing with smooth particles, i.e. the number of degrees of freedom reduces to 2 . 


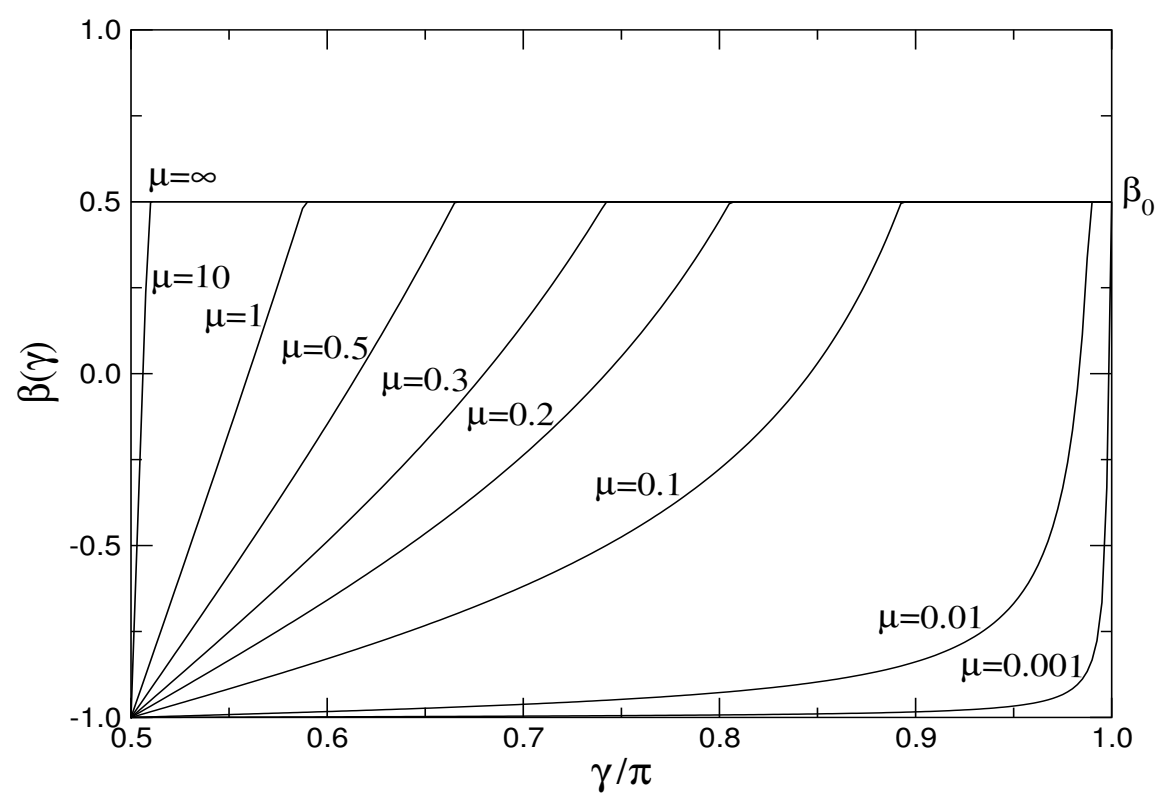

Figure 2.2.: Velocity dependent coefficient of tangential restitution as a function of the impact angle $\gamma$ for various values of the coefficient of Coulomb friction $\mu$.

\subsection{Homogeneous Bulk Driving}

We drive the system homogeneously by modifying the velocity of every particle at each time of agitation $t$ such that the velocity of the $i$-th particle changes according to

$$
\boldsymbol{v}_{i}^{\prime}(t)=\boldsymbol{v}_{i}(t)+v_{\mathrm{dr}} \boldsymbol{\xi}_{i}(t)
$$

where, once again, post-collisional quantities are primed. The driving velocity $v_{\mathrm{dr}}$ sets the time (velocity) scale and defines the driving temperature $T_{\mathrm{dr}}:=m v_{\mathrm{dr}}^{2}$. The components of the vector $\boldsymbol{\xi}_{i}(t)$, which are denoted by $\xi_{i, x}(t)$ and $\xi_{i, y}(\mathrm{t})$, are uncorrelated Gaussian random numbers with zero mean and variance

$$
\left\langle\xi_{i, k}(t) \xi_{j, l}\left(t^{\prime}\right)\right\rangle_{\{\xi\}}=\delta_{i j} \delta_{k l} \delta\left(t-t^{\prime}\right)
$$

Here $\delta_{i j}$ and $\delta_{k l}$ are Kronecker deltas and $\delta\left(t-t^{\prime}\right)$ is the Dirac delta function. The stochastic driving rule in Eq. (2.11) leads to an average rate of change of temperature

$$
\Delta T / \Delta t=H_{\mathrm{dr}}, \text { with } H_{\mathrm{dr}}=f_{\mathrm{dr}} T_{\mathrm{dr}}
$$

after every driving time step $\Delta t=f_{\mathrm{dr}}^{-1}$. 


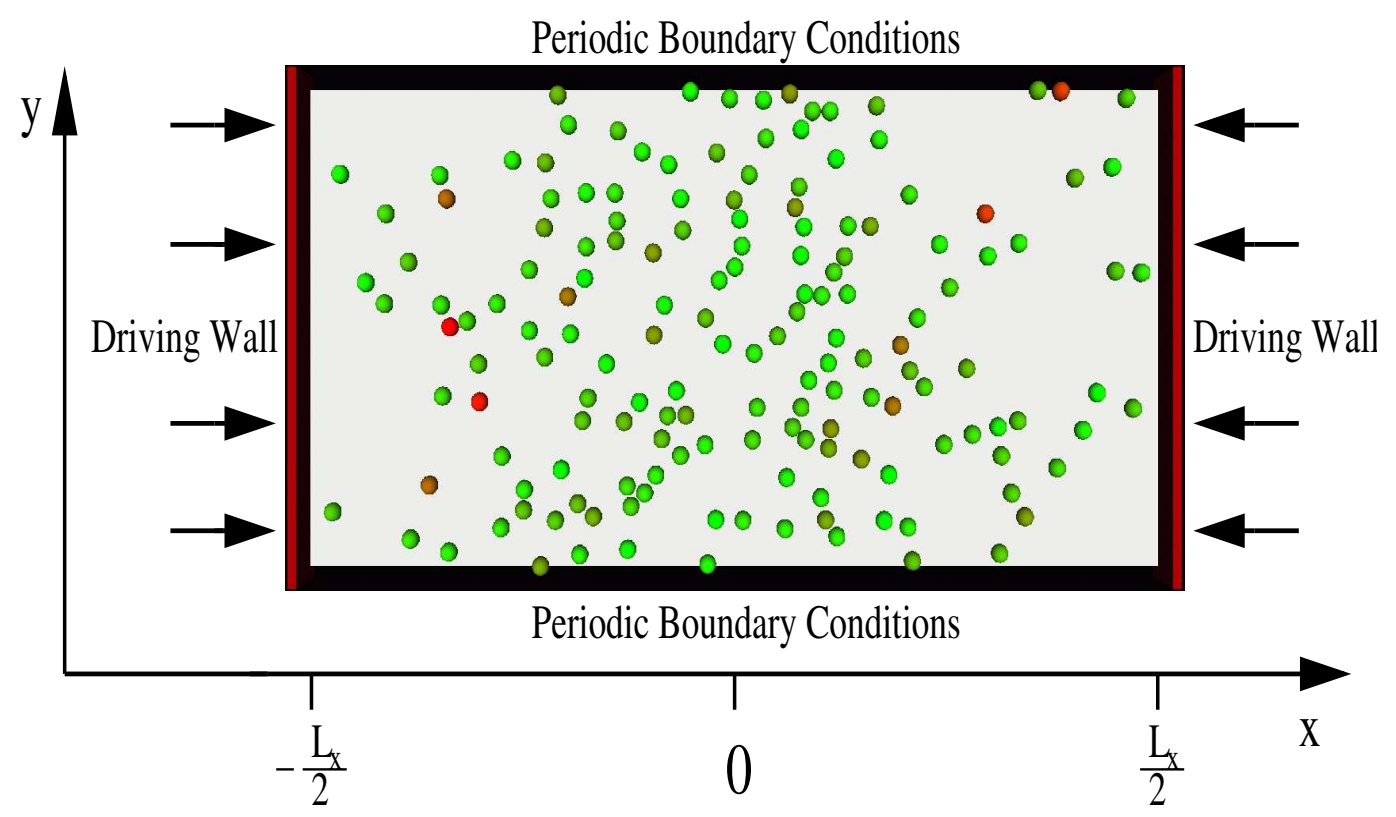

Figure 2.3.: Model of $\mathrm{N}$ disks, driven in the $x$-direction with periodic boundary conditions in the $y$ direction.

\subsection{Boundary Induced Driving}

We drive the system through the walls perpendicular to the $x$-direction while periodic boundary conditions are imposed in the $y$-direction. When a particle collides with a driving wall, energy is injected into the system. This can be modeled in different ways, for example by drawing a new velocity from a Maxwellian distribution of a given wall temperature [GZBN97] or by assuming that the wall has a coefficient of normal restitution that is greater than one. Both of these mechanisms have no close experimental equivalents, though. A more realistic model is to assume a vibrating wall moving either in a symmetric (e.g. sinusoidal) or in an asymmetric (e.g. saw-tooth) way [MB97, Kum98, Kum99a]. In addition, the walls can be inelastic and/or rough modeled by a normal and/or tangential coefficient of restitution for particle-wall collisions. Here we restrict ourselves to saw-tooth driving of smooth elastic walls in the limit of vanishing amplitudes $A$ and diverging frequency $\nu$ such that $A \nu=: v_{\mathrm{dr}} / 2$ is a constant. This ensures that the driving walls are always located at the same positions. Fig. 2.3 shows a typical snapshot. We will now write down the collision rules for collisions between a particle and an ideally vibrating wall for arbitrary inelasticity and roughness of the walls. To be even more general we will consider the 3D case first before we simplify it to two dimensions. These 3D collision rules are, in fact, the same as for binary collisions, Eqs. (2.4) and (2.5), where the particle representing the wall is considered to have infinite mass. This leads to the following simple 
expressions for the real particle's change of translational and rotational velocity, $\boldsymbol{v}$ and $\boldsymbol{\omega}$, respectively, due to a collision with wall $l$ (with normal vector $\hat{\boldsymbol{n}}_{l}$ pointing into the system)

$$
\boldsymbol{v}^{\prime}=\boldsymbol{v}+2 \Delta \boldsymbol{v}_{\mathrm{pw}}, \quad \omega^{\prime}=\omega+2 \Delta \omega_{\mathrm{pw}}
$$

where post-collisional quantities are primed and

$$
\begin{aligned}
& \boldsymbol{\Delta} \boldsymbol{v}_{\mathrm{pw}}=-\frac{\left(1+\alpha_{\mathrm{w}}\right)}{2}\left(\hat{\boldsymbol{n}}_{l} \cdot \boldsymbol{v}+v_{\mathrm{dr}}\right) \hat{\boldsymbol{n}}_{l}-\eta_{\mathrm{w}} \hat{\boldsymbol{n}}_{l} \times\left(\boldsymbol{v} \times \hat{\boldsymbol{n}}_{l}+\frac{a}{2} \boldsymbol{\omega}\right) \\
& \boldsymbol{\Delta} \boldsymbol{\omega}_{\mathrm{pw}}=\frac{2 \eta_{\mathrm{w}}}{q a}\left(\hat{\boldsymbol{n}}_{l} \times \boldsymbol{v}+\frac{a}{2} \hat{\boldsymbol{n}}_{l} \times\left(\hat{\boldsymbol{n}}_{l} \times \boldsymbol{\omega}\right)\right) .
\end{aligned}
$$

Here $\eta_{\mathrm{w}}=\eta_{\mathrm{w}}(\gamma):=q\left(1+\beta_{\mathrm{w}}(\gamma)\right) /(2(1+q)), q=(4 I) /\left(m a^{2}\right)$, and $v_{\mathrm{dr}} \geq 0$ is the velocity of the vibrating wall as described above. The three parameters used in the Walton model, $\alpha_{\mathrm{w}}$, $\mu_{\mathrm{w}}$, and $\beta_{0, \mathrm{w}}$ have the same meanings as the corresponding ones for binary collisions but may have different values. $\beta_{\mathrm{w}}(\gamma)$ is defined correspondingly, cf. Eq. (2.8).

Considering only walls in the $x$-direction $\Delta v_{\mathrm{pw}}$ and $\Delta \boldsymbol{\omega}_{\mathrm{pw}}$ can be written more explicitly for the left/right wall as

$$
\begin{aligned}
& 2 \boldsymbol{\Delta} \boldsymbol{v}_{\mathrm{pw}}=\left[-\left(1+\alpha_{\mathrm{w}}\right) v_{x} \pm v_{\mathrm{dr}}\right] \hat{\boldsymbol{e}}_{x}-2 \eta_{\mathrm{w}}\left(\left[v_{y}-\frac{a}{2} \omega_{z}\right] \hat{\boldsymbol{e}}_{y}+\left[v_{z}+\frac{a}{2} \omega_{y}\right] \hat{\boldsymbol{e}}_{z}\right) \\
& 2 \boldsymbol{\Delta} \boldsymbol{\omega}_{\mathrm{pw}}=\frac{4 \eta_{\mathrm{w}}}{q a}\left(\left[-v_{z}-\frac{a}{2} \omega_{y}\right] \hat{\boldsymbol{e}}_{y}+\left[v_{y}-\frac{a}{2} \omega_{z}\right] \hat{\boldsymbol{e}}_{z}\right) .
\end{aligned}
$$

In 2 dimensions all changes of the translational velocity that are proportional to $\hat{\boldsymbol{e}}_{z}$ vanish, i.e. the last term in the expression for $\Delta v_{\mathrm{pw}}$. Rotational changes, in contrast, must be proportional to $\hat{e}_{z}$ such that the first term in $\Delta \omega_{\mathrm{pw}}$ vanishes.

\section{Smooth Elastic Walls}

Even though the simulation developed is capable of running in 2 or 3 dimensions with or without gravity, with arbitrary inelasticity and roughness of the particles and the walls (or without walls) - and has been tested extensively in all regimes - all boundary driven simulations presented here will be for two dimensional zero-gravity systems of smooth particles with smooth elastic walls performing the saw-tooth driving described above. In that case the collision rule for a particle colliding with the left/right wall (in $x$-direction) simplifies further to

$$
v_{x}^{\prime}=-v_{x} \pm v_{\mathrm{dr}}, \quad v_{y}^{\prime}=v_{y},
$$

where, again, post-collisional quantities are primed. As will be discussed in detail in chapter 3 we will measure velocities in units of $v_{\mathrm{dr}}$ and masses in units of $m$. 


\section{Dimensional Analysis and Energy Balance}

The model system contains three independent length scales: the diameter $a$ of a disk and the sizes of the box edges $L_{x}$ and $L_{y}$. In addition, there is one independent velocity scale, the driving velocity $v_{\mathrm{dr}}$, and one independent mass scale, the mass of a disk $m$. Except for the initial positions and velocities of the particles there are no further dimensional quantities in this system that can be chosen and set independently. The stationary state is expected to be independent of the initial conditions. We will therefore measure all lengths in units of the particle diameter $a$, all times in units of $a / v_{\mathrm{dr}}$ and all energies in units of $m v_{\mathrm{dr}}^{2}$ in order to nondimensionalize the system. Note that there are no other time and energy scales. We thus introduce the following dimensionless variables: box sizes $\widetilde{L}_{x}=L_{x} / a$ and $\widetilde{L}_{y}=L_{y} / a$, granular temperatures $\widetilde{T}_{x}=T_{x} /\left(m v_{\mathrm{dr}}^{2}\right)$ and $\widetilde{T}_{y}=T_{y} /\left(m v_{\mathrm{dr}}^{2}\right)$, stress tensor (momentum flux density) $\widetilde{\boldsymbol{\sigma}}=\boldsymbol{\sigma} a^{2} /\left(m v_{\mathrm{dr}}^{2}\right)$, heat flux (energy flux density) $\widetilde{\boldsymbol{q}}=\boldsymbol{q} a^{2} /\left(m v_{\mathrm{dr}}^{3}\right)$, and local energy loss (dissipation density) $\widetilde{\zeta}=\zeta a^{3} /\left(m v_{\mathrm{dr}}^{3}\right)$. For (microscopic) definitions of these hydrodynamic fields the reader is referred to chapter 4 . In the stationary state all dimensionless observables like $\widetilde{T}, \widetilde{\boldsymbol{\sigma}}, \widetilde{\boldsymbol{q}}$, and $\widetilde{\zeta}$ are independent of the driving velocity and, in general, can only depend on the position $r$ and the remaining 6 independent dimensionless system parameters, which characterize the system completely: the number of disks $N$, the two edges of the system $\widetilde{L}_{x}$ and $\widetilde{L}_{y}$ in units of $a$, and the three dimensionless material constants: the coefficients of normal restitution $\alpha$, Coulomb friction $\mu$ (sliding), and tangential restitution $\beta_{0}$ (sticking).

Simulation results, starting in chapter 6 , will be presented using dimensionless units as described above. For simplicity of the notation we will refrain from indicating dimensionless quantities by a tilde. This should cause no confusion, because quantities having a physical dimension will no longer occur starting in chapter 6 (except for appendix $\mathrm{C}$ ).

In the following we distinguish between the two cases considered in this thesis: vibrated systems of smooth particles and homogeneously driven systems of rough particles with Coulomb friction. 


\subsection{Vibrated Systems of Smooth Particles}

Vibrated systems are investigated for smooth disks only, reducing the number of independent dimensionless system parameters to 4 . In addition, we are interested in the macroscopic limit (only), which is taken such that $N \rightarrow \infty$ and $L_{y} \rightarrow \infty$ with a fixed line density $\lambda:=N / L_{y}$ of particles. Thus, in the macroscopic limit the number of system parameters is further reduced to $L_{x}, \lambda$, and $\alpha$. It is important to keep $L_{x}$ finite, otherwise the driving would become a negligible marginal effect and energy balance would not work: energy input occurs only at the boundaries, while energy dissipation is a bulk phenomenon.

\section{Energy Balance}

It is instructive to estimate the average or global granular temperature $T:=\frac{m}{2 N} \sum_{i=1}^{N}\left|\boldsymbol{v}_{i}\right|^{2}$ by balancing the energy input at the walls and the energy loss due to particle collisions in the bulk. We do this in a slightly more general setting than needed for the simulations presented later and allow in addition for inelastic collisions with the perfectly smooth wall, characterized by a coefficient of restitution $\alpha_{\mathrm{w}}$. The appropriate generalization of the collision rule (2.17) includes both the driving velocity $v_{\mathrm{dr}}$ and the coefficient of restitution $\alpha_{\mathrm{w}}$ for particle-wall collisions. It can be deduced from Eq. (2.16) by setting $\eta_{\mathrm{w}}=0$ and reads

$$
v_{x}^{\prime}=-\alpha_{\mathrm{w}} v_{x} \pm v_{\mathrm{dr}}, \quad v_{y}^{\prime}=v_{y} .
$$

The special case $v_{\mathrm{dr}}=0$ and $\alpha_{\mathrm{w}}>1$ provides an alternative driving mechanism, which, however, does not give rise to a stationary state, as will be shown below.

The average energy gain $\Delta E_{\mathrm{pw}}$ due to a particle-wall collision is estimated from Eq. (3.1) by averaging the kinetic energy before and after the collision with a Maxwellian velocity distribution with (global) temperature $T$. This gives

$$
\Delta E_{\mathrm{pw}}=\frac{m}{2}\left(v_{\mathrm{dr}}^{2}+4 \alpha_{\mathrm{w}} \sqrt{\frac{T}{2 \pi m}} v_{\mathrm{dr}}-\left(1-\alpha_{\mathrm{w}}^{2}\right) \frac{T}{m}\right) .
$$

The collision frequency of particles with the left (right) wall is estimated by

$$
f_{\mathrm{pw}}=\frac{N}{L_{x}} \sqrt{\frac{T}{2 \pi m}},
$$

where we have assumed the density to be spatially homogeneous throughout the system.

When two disks collide in the bulk, the average change in total energy is computed similarly to (3.2) from (2.4) and (2.5) by averaging pre- and post-collisional kinetic energy, which yields

$$
\Delta E_{\mathrm{pp}}=-\frac{1-\alpha^{2}}{2} T .
$$


Finally, the number of particle-particle collisions per time is given approximately by Enskog's collision frequency [vNE98]

$$
f_{\mathrm{pp}}=\frac{N-1}{L_{x}} \lambda a \chi \sqrt{\frac{T \pi}{m}},
$$

where $\chi$ is the pair correlation function at contact of a corresponding elastic gas in thermal equilibrium. Since $\chi$ is not exactly known, we resort to the widely used Henderson approximation [Hen75]

$$
\chi \approx \frac{1-7 \phi / 16}{(1-\phi)^{2}}
$$

for numerical purposes. It may be viewed as a heuristic rational approximation to the virial expansion of $\chi$ and is the two-dimensional equivalent to the Carnahan-Starling approximation [CS69, HM86] for a three-dimensional hard-sphere gas. Additional higher-order terms to the Henderson approximation, which are proportional to $\phi^{3} /(1-\phi)^{4}$, have turned out to be irrelevant for our purposes and will therefore not be taken into account. Eq. (3.6) has been calculated for elastic systems. It has been found, though, the it is also a good approximation for inelastic systems [SM01].

Summing over energy loss in the bulk and energy gain at the right and left wall, and assuming $N-1 \approx N$, we obtain for the total change in granular temperature

$$
\frac{d T}{d t} \approx \frac{2 f_{\mathrm{pw}} E_{\mathrm{pw}}+f_{\mathrm{pp}} E_{\mathrm{pp}}}{N}=\frac{m}{L_{x}} \sqrt{\frac{T}{2 \pi m}}\left(v_{\mathrm{dr}}^{2}+4 \alpha_{\mathrm{w}} \sqrt{\frac{T}{2 \pi m}} v_{\mathrm{dr}}-\varepsilon_{\mathrm{eff}} \psi \frac{\pi}{2} \frac{T}{2 m}\right) .
$$

Here the effective dissipation coefficient

$$
\varepsilon_{\mathrm{eff}}:=\varepsilon+\frac{2}{\pi} \frac{1-\alpha_{\mathrm{w}}^{2}}{\psi}
$$

is given in terms of the coefficient of restitution for particle-wall collisions $\alpha_{\mathrm{w}}$ as well as

$$
\varepsilon:=1-\alpha^{2}, \quad \psi:=\sqrt{2} \chi \lambda .
$$

We briefly discuss two special cases:

a) For $v_{\mathrm{dr}}=0$ no stationary state is reached in general. Both energy gain (due to driving with $\alpha_{\mathrm{w}}>1$ ) and energy loss increase or decrease like $T^{3 / 2}$ depending on the sign of $\varepsilon_{\text {eff }}$, resulting in Haff's law

$$
\frac{d T}{d t}=-\frac{\varepsilon_{\text {eff }} \psi}{2 L_{x}} \sqrt{\frac{\pi}{2 m}} T^{3 / 2},
$$

which has been discussed extensively in the different context of freely cooling granular gases. Here the temperature continues to decrease $\left(\varepsilon_{\text {eff }}>0\right)$ or increase $\left(\varepsilon_{\text {eff }}<0\right)$ depending on whether dissipation or driving wins. This was also confirmed by simulations (not presented).

b) For $v_{\mathrm{dr}}>0$ and $\varepsilon_{\mathrm{eff}}>0$ the granular temperature adjusts to the driving so that the stationary state with $d T / d t=0$ is characterized by the quadratic equation

$$
m v_{\mathrm{dr}}^{2}+2 \alpha_{\mathrm{w}} v_{\mathrm{dr}} \sqrt{\frac{2 m T}{\pi}}-\varepsilon_{\mathrm{eff}} \psi \frac{\pi}{2} T=0
$$




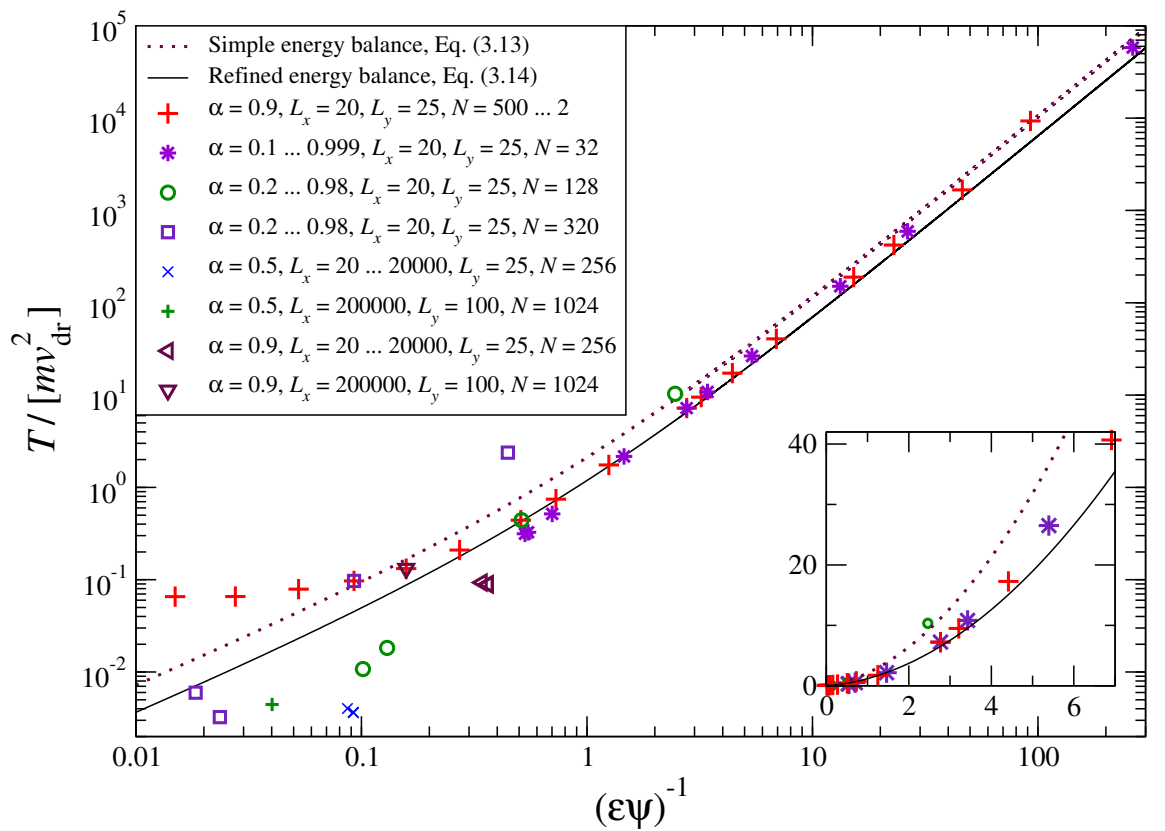

Figure 3.1.: Dimensionless global granular temperature $\frac{T}{m v_{\mathrm{dr}}^{2}}$ as a function of $(\varepsilon \psi)^{-1}$ defined in Eq. (3.9). Comparison between simulations and the simple energy-balance argument, Eq. (3.13), as well as with the refined version, Eq. (3.14). The inset shows the same graph but on on a non-logarithmic scale.

for the global temperature $T$. The solution of (3.11) is

$$
\frac{T}{m v_{\mathrm{dr}}^{2}}=\left(\frac{2}{\pi}\right)^{3}\left(\frac{\alpha_{\mathrm{w}}}{\varepsilon_{\mathrm{eff}} \psi}\right)^{2}\left(1+\sqrt{1+(\pi / 2)^{2} \varepsilon_{\mathrm{eff}} \psi / \alpha_{\mathrm{w}}^{2}}\right)^{2},
$$

which is independent of the initial data as expected. For $\alpha_{\mathrm{w}}=1$ this simplifies further to

$$
\frac{T}{m v_{\mathrm{dr}}^{2}}=\left(\frac{2}{\pi}\right)^{3}(\varepsilon \psi)^{-2}\left(1+\sqrt{1+(\pi / 2)^{2} \varepsilon \psi}\right)^{2},
$$

where $\varepsilon, \psi$, and $\chi$ are given in Eqs. (3.9) and (3.6).

In Fig. 3.1 we plot the prediction for the (dimensionless) global granular temperature $T /\left(m v_{\mathrm{dr}}^{2}\right)$ from Eq. (3.13) for systems driven by elastic walls as a function of $(\varepsilon \psi)^{-1}$ and compare it with simulations. For very small values of $(\varepsilon \psi)^{-1}$, corresponding to high density and/or high inelasticity, the simulations deviate significantly from this simple theory's prediction. For larger values, $(\varepsilon \psi)^{-1} \gtrsim 0.1$ the agreement is reasonable. In addition, we show the result

$$
\frac{T}{m v_{\mathrm{dr}}^{2}}=\frac{1}{2 \pi(\varepsilon \psi)^{2}}(1+\sqrt{1+2 \varepsilon \psi})^{2}
$$

of a more refined calculation, which uses a pseudo-Liouville-operator approach to kinetic theory which is shown in appendix C. Eq. (3.14) yields a better agreement with the data for intermediate values of $(\varepsilon \psi)^{-1}$. Please note the very simple argument in appendix C.6 (page 135) showing how to easily get from Eq. (3.13) to Eq. (3.14). 


\subsection{Homogeneously Driven Systems of Rough Particles with Coulomb Friction}

Homogeneously driven systems are investigated with periodic boundary conditions. Since we are interested in the macroscopic limit $N \rightarrow \infty, L_{x} \rightarrow \infty$, and $L_{y} \rightarrow \infty$ with a fixed number density $\rho_{0}=N /\left(L_{x} L_{y}\right)$ of particles, the number of system parameters further reduces to $\rho_{0}$, $\alpha, \mu$, and $\beta_{0}$ in the macroscopic limit.

Estimating the average global granular temperature is more difficult here because it is known [HZ97, LHMZ98] that equipartition of energy between translational and rotational degrees of freedom is violated for rough particles interacting dissipatively. This makes a simple energy balance argument similar to the one for smooth particles, as presented in the last section, almost as elaborate as the more refined version. For a discussion see page page 94 in chapter 10, Sec. 10.3.1. Details of the analytic calculation can be found in appendix $\mathrm{C}$. 


\section{Definition of Microscopic and Coarse-Grained Observables}

\subsection{Summary: Granular Hydrodynamics}

In this chapter we will show in detail how to microscopically calculate (measure) the hydrodynamic fields in an event-driven molecular dynamics simulation. The outcome will be the four hydrodynamic or balance equations (in 2 dimensions) plus exact expressions for the hydrodynamic fields (definitions will be given later in this chapter)

$$
\begin{aligned}
& 0=D_{t} \rho(\boldsymbol{r}, t)+\rho(\boldsymbol{r}, t) \nabla_{\boldsymbol{r}} \cdot \boldsymbol{V}(\boldsymbol{r}, t) \\
& 0=m \rho(\boldsymbol{r}, t) D_{t} V_{l}(\boldsymbol{r}, t)-\partial_{k} \sigma_{k l}(\boldsymbol{r}, t) \\
& 0=\rho(\boldsymbol{r}, t) D_{t} T(\boldsymbol{r}, t)+\nabla_{\boldsymbol{r}} \cdot \boldsymbol{q}(\boldsymbol{r}, t)-\zeta(\boldsymbol{r}, t)+\sigma_{k l}(\boldsymbol{r}, t) \partial_{k} V_{l}(\boldsymbol{r}, t)
\end{aligned}
$$

where Eq. (4.1) is the continuity or mass balance equation, (4.2) are the momentum balance equations, and (4.3) is the energy balance equation. $D_{t}:=\partial / \partial t+\boldsymbol{V}(\boldsymbol{r}, t) \cdot \nabla_{\boldsymbol{r}}$ denotes the material derivative. Eq. (4.1) is straight forward to see, the other ones will be derived in this chapter, including expressions for the stress tensor $\sigma$, the heat flux $\boldsymbol{q}$ and the energy sink $\zeta$.

\subsection{Microscopic Fields}

Let us define the microscopic fields similar to Ref. [GG01]. They can be expressed in terms of the microscopic distribution function

$$
f^{\text {mic }}(\boldsymbol{r}, \boldsymbol{v}, t):=\frac{1}{m} \sum_{i=1}^{N} m_{i} \delta\left(\boldsymbol{r}-\boldsymbol{r}_{i}(t)\right) \delta\left(\boldsymbol{v}-\boldsymbol{v}_{i}(t)\right)
$$

where $m:=\frac{1}{N} \sum_{i=1}^{N} m_{i}$, and $m_{i}, \boldsymbol{r}_{i}(t)$, and $\boldsymbol{v}_{i}(t)$ are the mass, position and velocity of particle $i$ at time $t$, respectively. In our simulations, all particles have the same mass, $m_{i}=m$, but it does not hurt to write down the more general case here. 
The microscopic mass density $\rho_{m}^{\text {mic }}(\boldsymbol{r}, t)$, momentum density $\boldsymbol{p}^{\text {mic }}(\boldsymbol{r}, t)$ and energy density $\mathcal{E}^{\text {mic }}(\boldsymbol{r}, t)$ are given as usual proportional to the zeroth, first, and second moment of the velocity, i.e. in 2 dimensions

$$
\begin{aligned}
\rho_{m}^{\mathrm{mic}}(\boldsymbol{r}, t) & :=m \int_{\mathbb{R}^{2}} d^{2} v f^{\mathrm{mic}}(\boldsymbol{r}, \boldsymbol{v}, t)=\sum_{i=1}^{N} m_{i} \delta\left(\boldsymbol{r}-\boldsymbol{r}_{i}(t)\right), \\
\boldsymbol{p}^{\mathrm{mic}}(\boldsymbol{r}, t) & :=m \int_{\mathbb{R}^{2}} d^{2} v f^{\mathrm{mic}}(\boldsymbol{r}, \boldsymbol{v}, t) \boldsymbol{v}=\sum_{i=1}^{N} m_{i} \boldsymbol{v}_{i}(t) \delta\left(\boldsymbol{r}-\boldsymbol{r}_{i}(t)\right) \\
\mathcal{E}^{\mathrm{mic}}(\boldsymbol{r}, t) & :=\frac{m}{2} \int_{\mathbb{R}^{2}} d^{2} v f^{\mathrm{mic}}(\boldsymbol{r}, \boldsymbol{v}, t)|\boldsymbol{v}|^{2}=\sum_{i=1}^{N} \frac{m_{i}}{2}\left|\boldsymbol{v}_{i}(t)\right|^{2} \delta\left(\boldsymbol{r}-\boldsymbol{r}_{i}(t)\right) \\
& =\sum_{i=1}^{N} E_{i}(t) \delta\left(\boldsymbol{r}-\boldsymbol{r}_{i}(t)\right) .
\end{aligned}
$$

Here $E_{i}(t)$ is the energy of particle $i$ at time $t$. Accordingly, we define the partial energy densities $\mathcal{E}_{x / y}^{\text {mic }}(\boldsymbol{r}, t):=\frac{m}{2} \int_{\mathbb{R}^{2}} d^{2} v f^{\text {mic }}(\boldsymbol{r}, \boldsymbol{v}, t) v_{x / y}^{2}$ and the number density

$$
\rho^{\mathrm{mic}}(\boldsymbol{r}, t):=\int_{\mathbb{R}^{2}} d^{2} v f^{\mathrm{mic}}(\boldsymbol{r}, \boldsymbol{v}, t)=\frac{1}{m} \rho_{m}^{\mathrm{mic}}(\boldsymbol{r}, t)
$$

If the particles are not perfectly smooth, they do not conserve their rotational velocity. This leads to one more degree of freedom in 2 dimensions. Thus, the distribution function $f$ mic becomes $f^{\text {mic }}(\boldsymbol{r}, \boldsymbol{v}, \boldsymbol{\omega}, t)$. Of course, in that case we would have to integrate over $d \omega$ in Eqs. (4.5) - (4.7), too. All those additional integrations yield 1. Furthermore, the energy density $\mathcal{E}^{\text {mic }}$ defined above changes its name to translational energy density $\mathcal{E}_{\mathrm{tr}}^{\text {mic }}$ and we define the angular momentum density $\boldsymbol{p}_{\text {rot }}^{\text {mic }}$ and the rotational energy density $\mathcal{E}_{\text {rot }}^{\text {mic }}$

$$
\begin{aligned}
\boldsymbol{p}_{\mathrm{rot}}^{\mathrm{mic}}(\boldsymbol{r}, t) & =I \int_{\mathbb{R}} d \omega f^{\mathrm{mic}}(\boldsymbol{r}, \boldsymbol{v}, \boldsymbol{\omega}, t) \boldsymbol{\omega}=\sum_{i=1}^{N} I_{i} \boldsymbol{\omega}_{i}(t) \delta\left(\boldsymbol{r}-\boldsymbol{r}_{i}(t)\right) \\
\mathcal{E}_{\mathrm{rot}}^{\mathrm{mic}}(\boldsymbol{r}, t) & =\frac{I}{2} \int_{\mathbb{R}} d \omega f^{\mathrm{mic}}(\boldsymbol{r}, \boldsymbol{v}, \boldsymbol{\omega}, t)|\boldsymbol{\omega}|^{2}=\sum_{i=1}^{N} \frac{I_{i}}{2}\left|\boldsymbol{\omega}_{i}(t)\right|^{2} \delta\left(\boldsymbol{r}-\boldsymbol{r}_{i}(t)\right) \\
& =\sum_{i=1}^{N} E_{\mathrm{rot}, i}(t) \delta\left(\boldsymbol{r}-\boldsymbol{r}_{i}(t)\right) .
\end{aligned}
$$

where $I:=\frac{1}{N} \sum_{i=1}^{N} I_{i}$. Here, $I_{i}$ is the moment of inertia of particle $i$ and $\boldsymbol{\omega}_{i}(t)$ is its rotational velocity at time $t$. The total energy density will thus be $\mathcal{E}^{\text {mic }}=\mathcal{E}_{\mathrm{tr}}^{\text {mic }}+\mathcal{E}_{\text {rot }}^{\text {mic }}$. Since hydrodynamic fields will be presented for smooth particles only, we will not consider $\boldsymbol{p}_{\text {rot }}^{\text {mic }}$ and $\mathcal{E}_{\text {rot }}^{\text {mic }}$ any further. Additionally, we no longer write down the explicit time dependence of the particles' positions and velocities to improve the readability. 


\subsection{Coarse-Graining the Fields}

\subsubsection{Densities of Mass, Momentum, and Energy, Velocity, and Temperature}

Like in Ref. [GG01] we introduce a local coarse-graining function $\Phi(\boldsymbol{r})$ such that $\int \Phi(\boldsymbol{r}) d^{2} r=1$ and define the coarse-grained distribution function as well as the local time-dependent mass density $\rho_{m}(\boldsymbol{r}, t)$, momentum density $\boldsymbol{p}(\boldsymbol{r}, t)$ and energy density $\mathcal{E}(\boldsymbol{r}, t)$

$$
\begin{aligned}
f(\boldsymbol{r}, \boldsymbol{v}, t) & :=\int \Phi\left(\boldsymbol{r}-\boldsymbol{r}^{\prime}\right) f^{\mathrm{mic}}\left(\boldsymbol{r}^{\prime}, \boldsymbol{v}, t\right) d^{2} r^{\prime}=\frac{1}{m} \sum_{i=1}^{N} m_{i} \Phi\left(\boldsymbol{r}-\boldsymbol{r}_{\boldsymbol{i}}\right) \delta\left(\boldsymbol{v}-\boldsymbol{v}_{\boldsymbol{i}}\right), \\
\rho_{m}(\boldsymbol{r}, t) & :=\int \Phi\left(\boldsymbol{r}-\boldsymbol{r}^{\prime}\right) \rho_{m}^{\mathrm{mic}}\left(\boldsymbol{r}^{\prime}, t\right) d^{2} r^{\prime}=\sum_{i=1}^{N} m_{i} \Phi\left(\boldsymbol{r}-\boldsymbol{r}_{\boldsymbol{i}}\right) \\
& =m \int_{\mathbb{R}^{2}} d^{2} v f(\boldsymbol{r}, \boldsymbol{v}, t), \\
\boldsymbol{p}(\boldsymbol{r}, t) & :=\int \Phi\left(\boldsymbol{r}-\boldsymbol{r}^{\prime}\right) \boldsymbol{p}^{\mathrm{mic}}\left(\boldsymbol{r}^{\prime}, t\right) d^{2} r^{\prime}=\sum_{i=1}^{N} m_{i} \boldsymbol{v}_{\boldsymbol{i}} \Phi\left(\boldsymbol{r}-\boldsymbol{r}_{\boldsymbol{i}}\right) \\
& =m \int_{\mathbb{R}^{2}} d^{2} v f(\boldsymbol{r}, \boldsymbol{v}, t) \boldsymbol{v}, \\
\mathcal{E}(\boldsymbol{r}, t) & :=\int \Phi\left(\boldsymbol{r}-\boldsymbol{r}^{\prime}\right) \mathcal{E}^{\mathrm{mic}}\left(\boldsymbol{r}^{\prime}, t\right) d^{2} r^{\prime}=\sum_{i=1}^{N} E_{i} \Phi\left(\boldsymbol{r}-\boldsymbol{r}_{\boldsymbol{i}}\right) \\
& =\frac{m}{2} \int_{\mathbb{R}^{2}} d^{2} v f(\boldsymbol{r}, \boldsymbol{v}, t)|\boldsymbol{v}|^{2} .
\end{aligned}
$$

The coarse-grained partial energy densities $\mathcal{E}_{x}(\boldsymbol{r}, t)$ and $\mathcal{E}_{y}(\boldsymbol{r}, t)$, and the coarse-grained number density $\rho(\boldsymbol{r}, t)$ are given correspondingly. The (coarse-grained) local mean velocity or velocity field is defined as

$$
\boldsymbol{V}(\boldsymbol{r}, t):=\boldsymbol{p}(\boldsymbol{r}, t) / \rho_{m}(\boldsymbol{r}, t)
$$

and the (coarse-grained) local temperature in two dimensions is defined as

$$
\begin{aligned}
\rho(\boldsymbol{r}, t) T(\boldsymbol{r}, t) & :=\frac{m}{2} \int_{\mathbb{R}^{2}} d^{2} v f(\boldsymbol{r}, \boldsymbol{v}, t)|\boldsymbol{v}-\boldsymbol{V}(\boldsymbol{r}, t)|^{2} \\
& =\mathcal{E}(\boldsymbol{r}, t)-\frac{1}{2} \rho_{m}(\boldsymbol{r}, t)|\boldsymbol{V}(\boldsymbol{r}, t)|^{2} .
\end{aligned}
$$

Again, the total temperature $T(\boldsymbol{r}, t)$ is the sum of the partial temperatures $T_{x}(\boldsymbol{r}, t)$ and $T_{y}(\boldsymbol{r}, t)$ given accordingly, $\rho(\boldsymbol{r}, t) T_{x / y}(\boldsymbol{r}, t)=\mathcal{E}_{x / y}(\boldsymbol{r}, t)-\frac{1}{2} \rho_{m}(\boldsymbol{r}, t) V_{x / y}^{2}(\boldsymbol{r}, t)$.

Note that the non-coarse-grained versions of the velocity field and the temperature are meaningless. The same is true for the hydrodynamic fields of the stress tensor, the heat flux and the energy sink that will be defined in the next section. 


\subsubsection{Stress Tensor}

The stress tensor $\sigma_{k l}(\boldsymbol{r}, t)$ consists of two parts, a kinetic or free streaming part $\sigma_{k l}^{\mathrm{kin}}(\boldsymbol{r}, t)$ and a collisional or potential one, that is due to interactions between the particles, $\sigma_{k l}^{\text {int }}(\boldsymbol{r}, t)$. In the following we will derive expressions for both parts.

Consider the change of the momentum density $\boldsymbol{p}(\boldsymbol{r}, t)$ in a time interval $\Delta t$ at fixed position $r$ where $\Delta t$ is thought to be infinitissimal but we keep the $\Delta$ to indicate that changes due to collisions are non-differentiable.

$$
\frac{\partial}{\partial t} \boldsymbol{p}(\boldsymbol{r}, t)=\sum_{i=1}^{N} m_{i} \boldsymbol{v}_{\boldsymbol{i}} \frac{\partial}{\partial t} \Phi\left(\boldsymbol{r}-\boldsymbol{r}_{\boldsymbol{i}}\right)+\frac{1}{\Delta t} \sum_{i=1}^{N}\left(\Delta\left[m_{i} \boldsymbol{v}_{\boldsymbol{i}}\right]\right) \Phi\left(\boldsymbol{r}-\boldsymbol{r}_{\boldsymbol{i}}\right)
$$

Using the summation convention, the $l$-th vector component of the first term can be re-written as

$$
\sum_{i=1}^{N} m_{i} v_{i, l} \frac{\partial}{\partial t} \Phi\left(\boldsymbol{r}-\boldsymbol{r}_{\boldsymbol{i}}\right)=-\frac{\partial}{\partial r_{k}}\left(\sum_{i=1}^{N} m_{i} v_{i, k} v_{i, l} \Phi\left(\boldsymbol{r}-\boldsymbol{r}_{\boldsymbol{i}}\right)\right)
$$

because the partial time derivative of $\Phi\left(\boldsymbol{r}-\boldsymbol{r}_{\boldsymbol{i}}\right)$ only acts on the $\boldsymbol{r}_{\boldsymbol{i}} \equiv \boldsymbol{r}_{i}(t)$ while the divergence acts on $\boldsymbol{r}$ but leaves the $\boldsymbol{r}_{\boldsymbol{i}}$ and $\boldsymbol{v}_{\boldsymbol{i}}$ untouched since the quantities of the $i$-th particle do not depend on the coarse-grained position $\boldsymbol{r}$. We now write $\boldsymbol{v}_{\boldsymbol{i}} \equiv \boldsymbol{v}_{i}(t)=\boldsymbol{V}(\boldsymbol{r}, t)+\tilde{\boldsymbol{v}}_{\boldsymbol{i}}(\boldsymbol{r}, t)$ where $\boldsymbol{V}(\boldsymbol{r}, t)$ is the local mean velocity as defined in Eq. (4.15) and $\tilde{\boldsymbol{v}}_{\boldsymbol{i}}(\boldsymbol{r}, t):=\boldsymbol{v}_{\boldsymbol{i}}-\boldsymbol{V}(\boldsymbol{r}, t)$ is the fluctuation velocity of particle $i$ compared to the (local) velocity field. In this notation we get $v_{i, k} v_{i, l}=\left(V_{k}(\boldsymbol{r}, t)+\tilde{v}_{i, k}(\boldsymbol{r}, t)\right)\left(V_{l}(\boldsymbol{r}, t)+\tilde{v}_{i, l}(\boldsymbol{r}, t)\right)=V_{k}(\boldsymbol{r}, t) V_{l}(\boldsymbol{r}, t)+\tilde{v}_{i, k}(\boldsymbol{r}, t) \tilde{v}_{i, l}(\boldsymbol{r}, t)+$ $V_{k}(\boldsymbol{r}, t) \tilde{v}_{i, l}(\boldsymbol{r}, t)+V_{l}(\boldsymbol{r}, t) \tilde{v}_{i, k}(\boldsymbol{r}, t)$. Since $\sum_{i=1}^{N} m_{i} \tilde{\boldsymbol{v}}_{\boldsymbol{i}}(\boldsymbol{r}, t) \Phi\left(\boldsymbol{r}-\boldsymbol{r}_{\boldsymbol{i}}\right)=0$ we get $\sum_{i=1}^{N} m_{i} v_{i, k} v_{i, l} \Phi\left(\boldsymbol{r}-\boldsymbol{r}_{\boldsymbol{i}}\right)=\rho_{m}(\boldsymbol{r}, t) V_{k}(\boldsymbol{r}, t) V_{l}(\boldsymbol{r}, t)+\sum_{i=1}^{N} m_{i} \tilde{v}_{i, k}(\boldsymbol{r}, t) \tilde{v}_{i, l}(\boldsymbol{r}, t) \Phi\left(\boldsymbol{r}-\boldsymbol{r}_{\boldsymbol{i}}\right)$.

Defining

$$
\sigma_{k l}^{\mathrm{kin}}(\boldsymbol{r}, t):=-\sum_{i=1}^{N} m_{i} \tilde{v}_{i, k}(\boldsymbol{r}, t) \tilde{v}_{i, l}(\boldsymbol{r}, t) \Phi\left(\boldsymbol{r}-\boldsymbol{r}_{\boldsymbol{i}}\right),
$$

and using the mass balance equation (4.1) and the material derivative $D_{t}:=\partial / \partial t+\boldsymbol{V}(\boldsymbol{r}, t)$. $\nabla_{\boldsymbol{r}}$, Eq. (4.17) can be written as

$$
\rho_{m}(\boldsymbol{r}, t) D_{t} \boldsymbol{V}(\boldsymbol{r}, t)=\frac{1}{\Delta t} \sum_{i=1}^{N}\left(\Delta \boldsymbol{p}_{\boldsymbol{i}}\right) \Phi\left(\boldsymbol{r}-\boldsymbol{r}_{\boldsymbol{i}}\right)+\frac{\partial}{\partial r_{k}} \sigma_{k l}^{\mathrm{kin}}(\boldsymbol{r}, t) \hat{\boldsymbol{e}}_{\boldsymbol{l}}
$$

(summation over $k$ and $l$ in the last term). Note that the kinetic part of the stress tensor $\sigma_{k l}^{\text {kin }}(\boldsymbol{r}, t)$ is symmetric. 
We will show now that $\frac{1}{\Delta t} \sum_{i=1}^{N}\left(\Delta \boldsymbol{p}_{\boldsymbol{i}}\right) \Phi\left(\boldsymbol{r}-\boldsymbol{r}_{\boldsymbol{i}}\right)$ can be written as the divergence of a tensor which we will identify as the collisional part of the stress tensor. Because we only have binary collisions we can write

$$
\frac{1}{\Delta t} \sum_{i=1}^{N}\left(\Delta \boldsymbol{p}_{\boldsymbol{i}}\right) \Phi\left(\boldsymbol{r}-\boldsymbol{r}_{\boldsymbol{i}}\right)=\frac{1}{\Delta t} \sum_{i=1}^{N} \sum_{\substack{j=1 \\ j \neq i}}^{N}\left(\Delta \boldsymbol{p}_{\boldsymbol{i} \mid \boldsymbol{j}}\right) \Phi\left(\boldsymbol{r}-\boldsymbol{r}_{\boldsymbol{i}}\right)
$$

where $\Delta \boldsymbol{p}_{\boldsymbol{i} \mid \boldsymbol{j}}$ is the change of momentum of particle $i$ due to a collision with particle $j$. Binary collisions due to the collision rule given in Eq. $(2.4,2.5)$ conserve momentum, $\Delta \boldsymbol{p}_{\boldsymbol{i} \mid \boldsymbol{j}}=$ $-\Delta \boldsymbol{p}_{j \mid \boldsymbol{i}}$. Therefore, the r.h.s. of Eq. (4.21) can be written as

$$
-\frac{1}{2 \Delta t} \sum_{i=1}^{N} \sum_{\substack{j=1 \\ j \neq i}}^{N}\left(\Delta \boldsymbol{p}_{\boldsymbol{i} \mid \boldsymbol{j}}\right)\left(\Phi\left(\boldsymbol{r}-\boldsymbol{r}_{\boldsymbol{j}}\right)-\Phi\left(\boldsymbol{r}-\boldsymbol{r}_{\boldsymbol{i}}\right)\right)
$$

Using the identity

$\nabla_{\boldsymbol{r}} \cdot\left(\boldsymbol{r}_{\boldsymbol{i j}} \int_{0}^{1} \Phi\left(\boldsymbol{r}-\boldsymbol{r}_{\boldsymbol{i}}+s \boldsymbol{r}_{\boldsymbol{i j}}\right) d s\right)=\int_{0}^{1} \frac{d}{d s} \Phi\left(\boldsymbol{r}-\boldsymbol{r}_{\boldsymbol{i}}+s \boldsymbol{r}_{\boldsymbol{i j}}\right) d s=\Phi\left(\boldsymbol{r}-\boldsymbol{r}_{\boldsymbol{j}}\right)-\Phi\left(\boldsymbol{r}-\boldsymbol{r}_{\boldsymbol{i}}\right)$

where $\boldsymbol{r}_{\boldsymbol{i} \boldsymbol{j}}:=\boldsymbol{r}_{\boldsymbol{i}}-\boldsymbol{r}_{\boldsymbol{j}}$, the term (4.22) can be written as $\frac{\partial}{\partial r_{l}} \sigma_{k l}^{\mathrm{int}}(\boldsymbol{r}, t) \hat{\boldsymbol{e}}_{\boldsymbol{k}}$ with

$$
\sigma_{k l}^{\mathrm{int}}(\boldsymbol{r}, t):=-\frac{1}{2 \Delta t} \sum_{i=1}^{N} \sum_{\substack{j=1 \\ j \neq i}}^{N}\left(\Delta p_{i \mid j, k}\right) r_{i j, l} \int_{0}^{1} \Phi\left(\boldsymbol{r}-\boldsymbol{r}_{\boldsymbol{i}}+s \boldsymbol{r}_{\boldsymbol{i}}\right) d s
$$

Note that - for smooth particles - the collisional part of the stress tensor is symmetric because the momentum transfer occurs along the connecting line between the two particles for all collisions. This means that every collision adds the same amount to $\sigma_{k l}$ as to $\sigma_{l k}$, leading to $\sigma_{k l} \equiv \sigma_{l k}$ (because the kinetic contribution is always symmetric).

To summarize, with the total stress tensor defined as $\boldsymbol{\sigma}(\boldsymbol{r}, t):=\boldsymbol{\sigma}^{\mathrm{kin}}(\boldsymbol{r}, t)+\boldsymbol{\sigma}^{\text {int }}(\boldsymbol{r}, t)$ the $l$-th vector component of the momentum density balance equation reads

$$
\rho_{m}(\boldsymbol{r}, t) D_{t} V_{l}(\boldsymbol{r}, t)=\partial_{k} \sigma_{k l}(\boldsymbol{r}, t)
$$

where $\partial_{k}:=\frac{\partial}{\partial r_{k}}$ and $k$ is assumed to be summed over all spatial dimensions. Using $\rho_{m}(\boldsymbol{r}, t)=$ $m \rho(\boldsymbol{r}, t)$ yields Eq. (4.2).

Practically measuring the stress tensor in the simulations, we face the problem that we do not know the final value (i.e. the time average of the stationary state) of the velocity field when we start measuring, i.e. we do not have the quantity $\tilde{\boldsymbol{v}}_{\boldsymbol{i}}(\boldsymbol{r}, t)$ but only $\boldsymbol{v}_{\boldsymbol{i}}(t)$ - and thus cannot measure $\boldsymbol{\sigma}^{\mathrm{kin}}(\boldsymbol{r}, t)$ as given in Eq. (4.19). Through direct measurement in the simulations only 
moments of the velocity distribution are accessible. Remembering that $\tilde{\boldsymbol{v}}_{\boldsymbol{i}}(\boldsymbol{r}, t)=\boldsymbol{v}_{\boldsymbol{i}}(t)-$ $\boldsymbol{V}(\boldsymbol{r}, t)$ we rewrite Eq. (4.19) as

$$
\sigma_{k l}^{\mathrm{kin}}(\boldsymbol{r}, t):=-\left(\sum_{i=1}^{N} m_{i} v_{i, k}(\boldsymbol{r}, t) v_{i, l}(\boldsymbol{r}, t) \Phi\left(\boldsymbol{r}-\boldsymbol{r}_{\boldsymbol{i}}\right)\right)-\rho(\boldsymbol{r}, t) V_{k}(\boldsymbol{r}, t) V_{l}(\boldsymbol{r}, t) .
$$

Here the kinetic part of the stress tensor is expressed solely in terms of moments of the velocity distribution, as it has been done for the temperature in the second line of Eq. (4.16).

\subsubsection{Heat Flux and Energy Sink}

Similar to the stress tensor the heat flux also has a kinetic and a collisional contribution. In addition we derive an expression for the local energy loss.

Consider the change of the energy density $\mathcal{E}(\boldsymbol{r}, t)$ in an (infinitissimal) time interval $\Delta t$ at fixed position $r$,

$$
\frac{\partial}{\partial t} \mathcal{E}(\boldsymbol{r}, t)=\sum_{i=1}^{N} E_{i} \frac{\partial}{\partial t} \Phi\left(\boldsymbol{r}-\boldsymbol{r}_{\boldsymbol{i}}\right)+\frac{1}{\Delta t} \sum_{i=1}^{N}\left(\Delta E_{i}\right) \Phi\left(\boldsymbol{r}-\boldsymbol{r}_{\boldsymbol{i}}\right) .
$$

The first term equals

$$
\sum_{i=1}^{N} E_{i} \frac{\partial}{\partial t} \Phi\left(\boldsymbol{r}-\boldsymbol{r}_{\boldsymbol{i}}\right)=-\nabla_{\boldsymbol{r}} \cdot\left(\sum_{i=1}^{N} E_{i} \boldsymbol{v}_{\boldsymbol{i}} \Phi\left(\boldsymbol{r}-\boldsymbol{r}_{\boldsymbol{i}}\right)\right)
$$

for the same reasons as has been discussed in the last section for Eq. (4.18). Here we write $E_{i}=\frac{m_{i}}{2}\left|\boldsymbol{V}(\boldsymbol{r}, t)+\tilde{\boldsymbol{v}}_{\boldsymbol{i}}\right|^{2}=\frac{m_{i}}{2}|\boldsymbol{V}(\boldsymbol{r}, t)|^{2}+\frac{m_{i}}{2}\left|\tilde{\boldsymbol{v}}_{\boldsymbol{i}}(\boldsymbol{r}, t)\right|^{2}+m_{i} \tilde{\boldsymbol{v}}_{\boldsymbol{i}}(\boldsymbol{r}, t) \cdot \boldsymbol{V}(\boldsymbol{r}, t)$. If, for the sake of simplicity, we assume $\boldsymbol{V}(\boldsymbol{r}, t)=0$ (the general case is treated in appendix A) the term within the parentheses at the right hand side of Eq. (4.28) can be written as

$$
\boldsymbol{q}^{\mathrm{kin}}(\boldsymbol{r}, t):=\sum_{i=1}^{N} \frac{m_{i}}{2}\left|\tilde{\boldsymbol{v}}_{\boldsymbol{i}}(\boldsymbol{r}, t)\right|^{2} \tilde{\boldsymbol{v}}_{\boldsymbol{i}}(\boldsymbol{r}, t) \Phi\left(\boldsymbol{r}-\boldsymbol{r}_{\boldsymbol{i}}\right)
$$

which we identify as the kinetic part of the heat flux. For non-zero velocity fields this definition is still valid while there will be additional terms within the parentheses at the right hand side of Eq. (4.28), cf. appendix A.

Using the definition of the temperature, Eq. (4.16), and the mass balance equation (4.1), both for $\boldsymbol{V}(\boldsymbol{r}, t)=0$, we get

$$
\rho(\boldsymbol{r}, t) \frac{\partial}{\partial t} T(\boldsymbol{r}, t)=\frac{1}{\Delta t} \sum_{i=1}^{N}\left(\Delta E_{i}\right) \Phi\left(\boldsymbol{r}-\boldsymbol{r}_{\boldsymbol{i}}\right)-\nabla_{\boldsymbol{r}} \cdot \boldsymbol{q}^{\mathrm{kin}}(\boldsymbol{r}, t) .
$$


Let us now take a look at $\frac{1}{\Delta t} \sum_{i=1}^{N}\left(\Delta E_{i}\right) \Phi\left(\boldsymbol{r}-\boldsymbol{r}_{\boldsymbol{i}}\right)$. It can be transformed into the divergence of the collisional part of the heat flux and the local energy loss term when we remember that we deal with instantaneous binary inelastic collisions. It is

$$
\frac{1}{\Delta t} \sum_{i=1}^{N}\left(\Delta E_{i}\right) \Phi\left(\boldsymbol{r}-\boldsymbol{r}_{\boldsymbol{i}}\right)=\frac{1}{\Delta t} \sum_{i=1}^{N} \sum_{\substack{j=1 \\ j \neq i}}^{N}\left(\Delta E_{i \mid j}\right) \Phi\left(\boldsymbol{r}-\boldsymbol{r}_{\boldsymbol{i}}\right)
$$

where $\Delta E_{i \mid j}$ is the change of energy of particle $i$ due to a collision with particle $j$. Writing $\Delta E_{i \mid j}=\frac{1}{2}\left(\Delta E_{i \mid j}+\Delta E_{j \mid i}\right)+\frac{1}{2}\left(\Delta E_{i \mid j}-\Delta E_{j \mid i}\right)$ we find that

$$
\begin{aligned}
& \frac{1}{\Delta t} \sum_{i=1}^{N}\left(\Delta E_{i}\right) \Phi\left(\boldsymbol{r}-\boldsymbol{r}_{\boldsymbol{i}}\right)=\frac{1}{2 \Delta t} \sum_{i=1}^{N} \sum_{\substack{j=1 \\
j \neq i}}^{N}\left(\Delta E_{i \mid j}+\Delta E_{j \mid i}\right) \Phi\left(\boldsymbol{r}-\boldsymbol{r}_{\boldsymbol{i}}\right) \\
& +\frac{1}{2 \Delta t} \sum_{i=1}^{N} \sum_{\substack{j=1 \\
j \neq i}}^{N}\left(\Delta E_{i \mid j}-\Delta E_{j \mid i}\right) \Phi\left(\boldsymbol{r}-\boldsymbol{r}_{\boldsymbol{i}}\right)
\end{aligned}
$$

The first term is the total local loss of energy $\zeta(\boldsymbol{r}, t)$ due to the sum of all collisions between particles $i$ and $j$,

$$
\zeta(\boldsymbol{r}, t):=\frac{1}{2 \Delta t} \sum_{i=1}^{N} \sum_{\substack{j=1 \\ j \neq i}}^{N}\left(\Delta E_{i \mid j}+\Delta E_{j \mid i}\right) \Phi\left(\boldsymbol{r}-\boldsymbol{r}_{\boldsymbol{i}}\right) .
$$

This term vanishes for elastic collisions, $\zeta(\boldsymbol{r}, t) \equiv 0$, because in the elastic case $\Delta E_{i \mid j}=$ $-\Delta E_{j \mid i}$. In Sec. 6.5 we show simulation results of $\zeta(\boldsymbol{r}, t)$ for the stationary state.

To show that the second term of the r.h.s. of Eq. (4.31) can be interpreted as the collisional part of the heat flux we note that it can be written as

$$
-\frac{1}{4 \Delta t} \sum_{i=1}^{N} \sum_{\substack{j=1 \\ j \neq i}}^{N}\left(\Delta E_{i \mid j}-\Delta E_{j \mid i}\right)\left(\Phi\left(\boldsymbol{r}-\boldsymbol{r}_{\boldsymbol{j}}\right)-\Phi\left(\boldsymbol{r}-\boldsymbol{r}_{\boldsymbol{i}}\right)\right) .
$$

Using again the identity (4.23) and $\boldsymbol{r}_{\boldsymbol{i}}:=\boldsymbol{r}_{\boldsymbol{i}}-\boldsymbol{r}_{\boldsymbol{j}}$, the term (4.33) can be written as $-\nabla_{\boldsymbol{r}}$. $\boldsymbol{q}^{\text {into }}(\boldsymbol{r}, t)$ with

$$
\boldsymbol{q}^{\text {into }_{\mathrm{o}}}(\boldsymbol{r}, t):=\frac{1}{4 \Delta t} \sum_{i=1}^{N} \sum_{\substack{j=1 \\ j \neq i}}^{N}\left(\Delta E_{i \mid j}-\Delta E_{j \mid i}\right) \boldsymbol{r}_{\boldsymbol{i}} \int_{0}^{1} \Phi\left(\boldsymbol{r}-\boldsymbol{r}_{\boldsymbol{i}}+s \boldsymbol{r}_{\boldsymbol{i}}\right) d s .
$$

Therefore, with $\boldsymbol{q}(\boldsymbol{r}, t):=\boldsymbol{q}^{\mathrm{kin}}(\boldsymbol{r}, t)+\boldsymbol{q}^{\text {into }}(\boldsymbol{r}, t)$ we can write for the case with a zero velocity field

$$
\rho(\boldsymbol{r}, t) \frac{\partial}{\partial t} T(\boldsymbol{r}, t)=-\nabla_{\boldsymbol{r}} \cdot \boldsymbol{q}(\boldsymbol{r}, t)+\zeta(\boldsymbol{r}, t)
$$


The general case of non-zero velocity fields is treated in appendix A. In agreement with the literature [BDS97, Gol03] the energy balance equation reads

$$
\rho(\boldsymbol{r}, t) D_{t} T(\boldsymbol{r}, t)=-\nabla_{\boldsymbol{r}} \cdot \boldsymbol{q}(\boldsymbol{r}, t)+\zeta(\boldsymbol{r}, t)-\sigma_{k l}(\boldsymbol{r}, t) \partial_{k} V_{l}(\boldsymbol{r}, t) .
$$

It is shown in appendix A that there is an additional term to the collisional part of the heat flux, cf. Eq. (A.7), which stems from the term in Eq. (4.28), $\boldsymbol{q}^{\text {intv }}(\boldsymbol{r}, t)=-\boldsymbol{\sigma}^{\text {int }}(\boldsymbol{r}, t) \cdot \boldsymbol{V}(\boldsymbol{r}, t)$. Thus the collisional part of the heat flux for non-zero velocity fields reads

$$
\boldsymbol{q}^{\mathrm{int}}(\boldsymbol{r}, t)=\boldsymbol{q}^{\mathrm{int}_{\mathrm{o}}}(\boldsymbol{r}, t)+\boldsymbol{q}^{\mathrm{int}_{\mathrm{v}}}(\boldsymbol{r}, t) .
$$

For practical measurements it is necessary again to write the kinetic contribution of the heat flux in terms of moments of the velocity distribution. When we put everything together we get for the total heat flux

$$
\begin{aligned}
\boldsymbol{q}(\boldsymbol{r}, t)= & \boldsymbol{q}^{\mathrm{kin}}(\boldsymbol{r}, t)+\boldsymbol{q}^{\mathrm{into}_{\mathrm{o}}}(\boldsymbol{r}, t)+\boldsymbol{q}^{\mathrm{int}_{\mathrm{v}}}(\boldsymbol{r}, t) \\
= & \sum_{i=1}^{N} \frac{m_{i}}{2}\left|\boldsymbol{v}_{\boldsymbol{i}}\right|^{2} \boldsymbol{v}_{i} \Phi\left(\boldsymbol{r}-\boldsymbol{r}_{i}\right)+\boldsymbol{q}^{\mathrm{int}_{\mathrm{o}}}(\boldsymbol{r}, t) \\
& -\mathcal{E}(\boldsymbol{r}, t) \boldsymbol{V}(\boldsymbol{r}, t)+m \rho(\boldsymbol{r}, t)|\boldsymbol{V}(\boldsymbol{r}, t)|^{2} \boldsymbol{V}(\boldsymbol{r}, t)-\boldsymbol{\sigma}(\boldsymbol{r}, t) \cdot \boldsymbol{V}(\boldsymbol{r}, t) .
\end{aligned}
$$

Here the heat flux $\boldsymbol{q}(\boldsymbol{r}, t)$ has been expressed solely in terms of moments of the velocity distribution, collisional terms ( $q^{\text {into }}$ is given in Eq. (4.34)) and $\boldsymbol{\sigma}(\boldsymbol{r}, t)$. Note that $\boldsymbol{\sigma}(\boldsymbol{r}, t)$ includes kinetic and collisional contributions. Details of the calculation can be found in appendix A. 


\section{Measuring Observables in Simulations}

\subsection{Event Driven Simulations of Vibrated Systems}

Following, e.g., Refs. [Rap88, Lub91, LHB94, LM98, HHZ00] we performed Event Driven (ED) simulations in two dimensions with periodic boundary conditions in the $y$-direction. In the $x$-direction we used fixed walls. An ED simulation is a specialized and very efficient Molecular Dynamics (MD) simulation, i.e. we numerically integrate Newton's equations of motion. In a standard MD simulation this is done by simultaneously advancing all particles by a sufficiently small time step. In an ED simulation we check in an efficient way [Lub91] which collision event will take place next and advance the involved particles exactly up to the instant of the collision. We then update those particles' velocities according to the collision rules (2.4) or (2.14) and proceed to the next collision event. This means that we analytically integrate the time between collision events and numerically find the next collision event. Here the average time step is of the order of the time between two collisions, and it varies from collision to collision. That allows for much larger (average) time steps than in standard MD simulations, where the time step chosen must be small enough to avoid particles overlapping significantly at any instant of time. In particular, the time steps must be much smaller than the average time between two collisions. In an ED simulation, by construction, the particles can never overlap. That means that the ED algorithm is suitable for binary collisions of hard core particles only - it cannot simulate multi-particle contacts and it does not work if there are forces between the particles. Of course, forces that do not depend on the position of the other particles, e.g. gravity, can be included if it is possible to integrate them analytically.

While a naive way of finding the next collision consumes CPU time proportional to $N^{2}$ (where $N$ is the number of particles) using a heap tree requires CPU time proportional to $N \ln N$ only, cf. [Lub91]. Subdividing the total box into smaller sub-boxes that contain only a few particles each further accelerates the algorithm. Collisions with walls and crossing the boundary between sub-boxes count as (collision) events, too. Details of the algorithm used can be found in Refs. [Lub91, HHZ00] and references therein. The problem of inelastic collapse characteristic of the ED algorithm [MY92, MY94, MBD94, CGM95, GZ96, EP97, GSB ${ }^{+} 98$, Gol03], is handled by using normal restitution coefficients that depend on the time elapsed since the last collision event (TC model) [LCRD96, LM98, LG03]. If a particle collides twice within a very short time interval the second collision is assumed to be elastic. 
We initialize the system by placing the particles on a triangular lattice with a Gaussian velocity distribution. To let the correlations of the initial state relax, the system is evolved elastically with periodic boundary conditions in the $y$-direction and elastic non-vibrating walls in the $x$ direction for an average of 100 collisions per particle. Then we switch on the driving of the left and right wall and the dissipation for particle-particle-collisions. Before we start measuring the observables we let the system relax further until, at time $t_{0}$, it has reached a stationary state, as indicated by the total kinetic energy, which fluctuates around a time-independent mean value.

\subsection{Details of the Measurement of the Kinetic and Collisional Contributions}

\subsubsection{In the Bulk}

Hydrodynamic fields in the stationary state are measured by subdividing the box into cells $\mathcal{V}_{\boldsymbol{r}}$ of area $\left|\mathcal{V}_{\boldsymbol{r}}\right|$, centered at position $\boldsymbol{r}=(x, y)$. In our simulations we choose the cells $\mathcal{V}_{\boldsymbol{r}}$ as narrow stripes along the $y$-direction (for a discussion see below). To measure the distribution function, for example, we count the number of particles $f\left(\boldsymbol{r}, v_{x}, v_{y}, t\right)\left|\mathcal{V}_{\boldsymbol{r}}\right| d v_{x} d v_{y}$ at time $t$ in cell $\mathcal{V}_{\boldsymbol{r}}$ with an $x$-component of the velocity between $v_{x}$ and $v_{x}+d v_{x}$ and $y$-component of the velocity between $v_{y}$ and $v_{y}+d v_{y}$.

Local observables fluctuate as a function of time. To eliminate these fluctuations of an observable $A(\boldsymbol{r}, t)$ we average over a (long) time interval of length $\tau$ and compute

$$
A^{\text {stat }}(\boldsymbol{r}):=\frac{1}{\tau} \int_{t_{0}}^{t_{0}+\tau} d t A(\boldsymbol{r}, t)
$$

as a Riemann sum by repeating single measurements at fixed time intervals, summing them up and dividing by the number of measurements.

Speaking in terms of Sec. 4.3 .3 we choose

$$
\Phi(\boldsymbol{r})=\frac{\Theta\left(|x|-\mathcal{L}_{x} / 2\right) \Theta\left(|y|-\mathcal{L}_{y} / 2\right)}{\left|\mathcal{V}_{\boldsymbol{r}}\right|}
$$

which means that the size of the cell is given by $\left|\mathcal{V}_{\boldsymbol{r}}\right|=\mathcal{L}_{x} \mathcal{L}_{y}$. We choose narrow $\left(\mathcal{L}_{x} \ll\right.$ 1) stripes along the $y$-axis $\left(\mathcal{L}_{y}=L_{y}\right)$. The coarse-grained positions are denoted by $\boldsymbol{r}=$ $(x, y)$. When measuring the collisional contributions, we do not calculate the integral $I:=$ $\int_{0}^{1} \Phi\left(\boldsymbol{r}-\boldsymbol{r}_{\boldsymbol{i}}+s \boldsymbol{r}_{\boldsymbol{i} \boldsymbol{j}}\right) d s$ for each collision. Instead we use $I=1 /\left(2\left|\mathcal{V}_{\boldsymbol{r}}\right|\right)$ for those cells in which the center of mass of exactly one particle is located at the instant of the collision. Should both particles' centers of mass be located in the same cell then this cell is assigned weight $I=1 /\left|\mathcal{V}_{\boldsymbol{r}}\right|$, for all other cells $I=0$. Because we are interested in long-time averages only, the error we make in a single measurement averages out for long times. 
Coarse-grained measurements in space and time of certain observables may depend on the coarse-graining resolution. For example, this was demonstrated for the stress tensor in shearflow driven granular systems in Ref. [Gol98, GG01]. Our measurements of observables in the stationary state, however, should not suffer from such effects for two reasons: First, we could not detect any significant non-zero local velocity field in the simulated systems and, second, because of the long-time average needed to obtain stationary-state quantities.

Subdividing the box not only in the $x$-direction but also in the $y$-direction, we have never found any significant dependence of the (long-time-averaged) hydrodynamic fields on $y$, the coordinate parallel to the driving walls. Yet, stripe states, which are homogeneous in $y$, but have an enhanced density in the middle of the sample, are known to exhibit instabilities with respect to density fluctuations in $y$ [LMS02a, LMS02b]. A marginal stability analysis [LMS02b] of granular hydrodynamics (for $\alpha$ close to one) determines the conditions under which such phenomena occur. As far as a comparison can be made, our systems fall into the stable region. Hence, we choose the cells $\mathcal{V}_{\boldsymbol{r}}$ as stripes along the $y$-direction, for which we write $\mathcal{V}_{x}$, and compute the hydrodynamic fields with spatial resolution in $x$-direction only. Thus, we also write $\rho(x)$ instead of $\rho(\boldsymbol{r})$ and change the notation accordingly for all other quantities. In the simulations the stripes $\mathcal{V}_{x}$ were all chosen to have equal width $L_{x} / 201$, the temporal resolution of our measurements was set to $\Delta t=1$, and the long-time average typically involves $10^{7}$ $10^{9}$ collision events.

\section{Kinetic Contributions}

As already mentioned in chapter 4 all kinetic contributions can be expressed in terms of moments of the velocity distribution. To measure the $k$-th moment $\mathcal{M}_{k, i}(\boldsymbol{r}, t)$ of the local distribution of the $i$-th component ( $i=x$ or $i=y$ ) of the velocity $v_{i}$ at time $t$ we sum $v_{i}^{k}$ over all particles in $\mathcal{V}_{\boldsymbol{r}}$ and divide it by the size of the cell $\left|\mathcal{V}_{\boldsymbol{r}}\right|, \mathcal{M}_{k, i}(\boldsymbol{r}, t):=\frac{1}{\left|\mathcal{V}_{\boldsymbol{r}}\right|} \sum_{n} v_{n, i}^{k}(t)$. Here, $v_{n, i}^{k}$ is the $i$-th component of the velocity of particle $n$ taken to the power of $k$. The sum over $n$ runs over all particles in cell $\mathcal{V}_{\boldsymbol{r}}$. Similarly we define the $k$-th moment $\mathcal{M}_{k}(\boldsymbol{r}, t)$ of the local distribution of the velocity $\boldsymbol{v}$ at time $t, \mathcal{M}_{k}(\boldsymbol{r}, t):=\frac{1}{\left|\mathcal{V}_{\boldsymbol{r}}\right|} \sum_{n}\left|\boldsymbol{v}_{n}(t)\right|^{k}$.

Of particular interest are the particle density $\rho(\boldsymbol{r})=\mathcal{M}_{0}(\boldsymbol{r})$ or the area fraction $\phi(\boldsymbol{r}):=$ $\rho(\boldsymbol{r}) \pi a^{2} / 4$ and the two components $i=x, y$ of the granular temperatures $T_{i}(\boldsymbol{r})=$ $\frac{m}{\mathcal{M}_{0}}(\boldsymbol{r})\left(\mathcal{M}_{2, i}(\boldsymbol{r})-\mathcal{M}_{1, i}^{2}(\boldsymbol{r})\right)$. The total granular temperature in 2 dimensions is, as defined above, $T(\boldsymbol{r}):=\left(T_{x}(\boldsymbol{r})+T_{y}(\boldsymbol{r})\right) / 2$.

The kinetic parts of the stress tensor and heat flux can be measured accordingly, see Eqs. (4.26) and (4.38). 


\section{Collisional Contributions}

In addition to the kinetic contributions both the stress tensor and the heat flux have contributions due to interactions of the particles, see Sec. 4.3.3. Here we show how to implement the measurements of the collisional contributions in our simulations.

Let us first consider the stress tensor. Suppose there is a collision at time $t$ of particle $k$ with another particle, then the momentum change of particle $k$ will contribute to the stress tensor an amount proportional to $l_{i}^{k}(t) \Delta p_{j}^{k}(t)$. Here $l_{i}^{k}(t)$ is the $i$-th component of the vector of length $a / 2$ pointing from the center of disk $k$ to its collision contact point, and $\Delta p_{j}^{k}(t)$ is the $j$-th component of the momentum change of particle $k$ during this collision. To compute the collisional part of the stress tensor we need the total change of momentum in the time interval $[t-\Delta t, t]$ in the cell $\mathcal{V}_{\boldsymbol{r}}$ so that we have to keep track of all collisions $n$ occurring at times $t_{n} \in[t-\Delta t, t]$, for which at least one collision partner $k_{n}$ (i.e. its center of mass) is located in cell $\mathcal{V}_{\boldsymbol{r}}$ at time $t_{n}$ [LM98]

$$
\sigma_{i j}^{\mathrm{int}}(\boldsymbol{r}, t)=\frac{1}{\Delta t} \frac{1}{\left|\mathcal{V}_{\boldsymbol{r}}\right|} \sum_{t_{n}} \sum_{k_{n}} l_{i}^{k_{n}}\left(t_{n}\right) \Delta p_{j}^{k_{n}}\left(t_{n}\right)
$$

The particle number $k_{n}$ of each such collision can take on one or two values, depending on whether one or both collision partners are located in cell $\mathcal{V}_{\boldsymbol{r}}$. Similar to the other hydrodynamic fields, in a second step $\boldsymbol{\sigma}^{\text {int }}(\boldsymbol{r}, t)$ is averaged over time in order to get the collisional part of the stress tensor in the stationary state, cf. Eq. (5.1). The corresponding local pressure $p(\boldsymbol{r}):=-\operatorname{tr}(\boldsymbol{\sigma}(\boldsymbol{r})) / 2$ is defined as usual as the negative trace of the stress tensor divided by the space dimension.

Computing the collisional part of the heat flux vector according to Eq. (4.34) in Sec. 4.3.3 is very similar to the collisional part of the stress tensor. We need the change of energy in the time interval $[t-\Delta t, t]$ in the cell $\mathcal{V}_{\boldsymbol{r}}$ so that, again, we have to keep track of all collisions $n$ occurring at times $t_{n} \in[t-\Delta t, t]$, for which at least one collision partner $k_{n}$ (i.e. its center of mass) is located in cell $\mathcal{V}_{\boldsymbol{r}}$ at time $t_{n}$,

$$
q_{i}^{\text {into }_{\mathrm{o}}}(\boldsymbol{r}, t)=-\frac{1}{\Delta t} \frac{1}{\left|\mathcal{V}_{\boldsymbol{r}}\right|} \sum_{t_{n}} \sum_{k_{n}} l_{i}^{k_{n}}\left(t_{n}\right) \frac{1}{2}\left(\Delta E^{k_{n} \mid \widetilde{k}_{n}}\left(t_{n}\right)-\Delta E^{\widetilde{k}_{n} \mid k_{n}}\left(t_{n}\right)\right)
$$

where $\widetilde{k}_{n}$ is the collision partner of $k_{n}, \Delta E^{k_{n} \mid \widetilde{k}_{n}}\left(t_{n}\right)=\frac{m}{2}\left(\boldsymbol{v}_{k_{n}}^{\prime 2}-\boldsymbol{v}_{k_{n}}^{2}\right)$ is the change of energy of particle $k_{n}$ due to the collision with particle $\widetilde{k}_{n}$, and $\frac{1}{2}\left(\Delta E^{k_{n} \mid \widetilde{k}_{n}}\left(t_{n}\right)-\Delta E^{\widetilde{k}_{n} \mid k_{n}}\left(t_{n}\right)\right)=$ $-\frac{m}{2} \frac{1+\alpha}{2}\left(\hat{\boldsymbol{n}} \cdot \boldsymbol{v}_{k_{n} \widetilde{k}_{n}}\right)\left(\hat{\boldsymbol{n}} \cdot\left[\boldsymbol{v}_{k_{n}}+\boldsymbol{v}_{\widetilde{k}_{n}}\right]\right)$. Again, the particle number $k_{n}$ of each such collision can take on one or two values depending on whether one or both collision partners are located in cell $\mathcal{V}_{\boldsymbol{r}}$. Again, $\boldsymbol{q}^{\mathrm{into}_{\mathrm{o}}}(\boldsymbol{r}, t)$ is averaged over time according to Eq. (5.1). 
The local energy loss term has only a collisional part. It is given by Eq. (4.32) which translates to

$$
\zeta(\boldsymbol{r}, t)=\frac{1}{\Delta t} \frac{1}{\left|\mathcal{V}_{\boldsymbol{r}}\right|} \sum_{t_{n}} \sum_{k_{n}} \frac{1}{2}\left(\Delta E^{k_{n} \mid \widetilde{k}_{n}}\left(t_{n}\right)+\Delta E^{\widetilde{k}_{n} \mid k_{n}}\left(t_{n}\right)\right)
$$

where $\frac{1}{2}\left(\Delta E^{k_{n} \mid \widetilde{k}_{n}}\left(t_{n}\right)+\Delta E^{\widetilde{k}_{n} \mid k_{n}}\left(t_{n}\right)\right)=-\frac{m}{2} \frac{1-\alpha^{2}}{4}\left(\hat{\boldsymbol{n}} \cdot \boldsymbol{v}_{k_{n} \widetilde{k}_{n}}\right)^{2}$.

\subsubsection{At the Wall}

\section{Collisional Contributions}

We also want to measure these quantities exactly at the wall. The collisional part can be computed almost as described above, by assuming the wall to be a particle of infinite mass. Then, in Eqs. (5.5), (5.4), and (5.3), we sum over the (real) particles only - and leave out the wall. To account for the fact that the sum no longer runs over each particle twice, each event has to be assigned twice the weight. Thus, the energy loss term, Eq. (5.5), at the left/right wall - which is a gain term for driving walls, of course - reads

$$
\zeta\left(\mp L_{x} / 2\right)=\frac{1}{\tau L_{y}} \sum_{t_{n}} \Delta E^{k_{n} \mid \text { wall }}\left(t_{n}\right) .
$$

Here we have used $\tau$ instead of $\Delta t$ to indicate that we are already talking about the timeaveraged (and $y$-independent) quantities in the stationary state. For the collision rule used for particle-wall collisions in the simulations presented later, Eq. (2.17), this simplifies to

$$
\zeta\left(\mp L_{x} / 2\right)=\frac{1}{\tau L_{y}} \sum_{\text {colls }}\left(v_{x}^{\prime 2}-v_{x}^{2}\right),
$$

where $v_{x}^{\prime}$ is the $x$-component of the particle's post-collisional velocity. The collisional part of the heat flux, Eq. (5.4), at the wall becomes

$$
q_{i}^{\text {into }_{o}}\left(\mp L_{x} / 2\right)=-\frac{1}{\tau L_{y}} \sum_{t_{n}} l_{i}^{k_{n}}\left(t_{n}\right) \Delta E^{k_{n} \mid \text { wall }}\left(t_{n}\right) .
$$

which simplifies to $q_{y}^{\text {into }}\left(\mp L_{x} / 2, t\right)=0$ and

$$
q_{x}^{\text {into }_{\mathrm{o}}}\left(\mp L_{x} / 2\right)= \pm \frac{1}{2} \frac{1}{\tau L_{y}} \frac{m}{2} \sum_{\text {colls }}\left(v_{x}^{\prime 2}-v_{x}^{2}\right) .
$$

for the collision rule used in this paper, Eq. (2.17), since $l_{x}^{k_{n}}\left(t_{n}\right)=\mp 1 / 2$ for the left/right wall while $l_{y}^{k_{n}}\left(t_{n}\right)=0$.

Similarly the collisional part of the stress tensor exactly at the wall can be obtained. 


\section{Kinetic Contributions}

One could be tempted to think that there is only a collisional contribution to any observable exactly at the wall. But there is a second part, which could be called its kinetic contribution directly at the wall. It arises when a distribution density is calculated from the distribution of collisions with the wall and has to be taken into account in a way similar to the measurement of the velocity distribution directly at the wall (see chapter 9). The basic idea is to define a virtual box of size $L_{y} \Delta x$ around the wall and calculate how much time a particle spends within the box before and after hitting the wall. This defines the weights assigned to the particle's preand post-collisional kinetic contributions to the observable.

Consider the contribution of a single particle to the time average of an observable $A$ per unit cell in a cell of size $L_{y} \Delta x$,

$$
\frac{1}{\tau L_{y} \Delta x} \int A\left(v_{x}, v_{y}\right) B(t) d t
$$

where $\tau$ is the averaging time interval and $B(t)$ is the characteristic function, i.e. $B(t)=1$ while the particle is within the cell and zero, otherwise. Let us first consider the cell to be in the bulk, far away from the walls. Choose the total time interval such that the particle passes the cell exactly once and let $\Delta x$ be small enough such that no collision occurs while the particle is within the cell. In this case the integral simply yields $A\left(v_{x}, v_{y}\right) \Delta t$ where $\Delta t$ is the time the particle spends within the cell, i.e. $\Delta t=\Delta x /\left|v_{x}\right|$. That means that this particle's contribution to $A$ is assigned a weight $1 /\left|v_{x}\right|$, where $v_{x}$ is the $x$-component of its own (constant) velocity while in the cell.

Now consider the stripe $\Delta x$ to be confined by a wall on one side and choose the magnitude of $\Delta x$ such that the particle will encounter no collisions within $\Delta x$ (neither before nor after hitting the wall). The wall is considered outside $\Delta x$. The time interval $\tau$ is chosen long enough such that the particle will enter $\Delta x$, hit the wall, and leave $\Delta x$ within this time interval (thus passing $\Delta x$ twice). Here, the contribution of the single particle to the time average of observable $A$ per unit cell will be

$$
\frac{1}{\tau L_{y} \Delta x} \int A\left(v_{x}, v_{y}\right) B(t) d t=\frac{1}{\tau L_{y}}\left(\frac{A\left(v_{x}, v_{y}\right)}{\left|v_{x}\right|}+\frac{A\left(v_{x}^{\prime}, v_{y}^{\prime}\right)}{\left|v_{x}^{\prime}\right|}\right)
$$

where the unprimed quantities $v_{x}$ and $v_{y}$ denote the particle's velocity components prior to the collision with the wall while $v_{x}^{\prime}$ and $v_{y}^{\prime}$ are the post-collisional quantities. To average the observable $A$ over a long time interval $\tau$ we have to sum up the right hand side of Eq. (5.11) for all particles colliding with the wall during the time interval $\tau$.

Finally, consider the wall to be inside the stripe of width $\Delta x$. Since $\Delta x$ will eventually be taken to zero, it must be centered around the wall. This means that, compared to the situation above, the particle will spend only half the time within $\Delta x$ - both before and after hitting the 
wall - because only a stripe of width $\Delta x / 2$ is accessible to the particle. The other half of the stripe is behind the wall. This yields a kinetic contribution that is a factor of 2 smaller than in the case, where the wall is not within the box but immediately outside of it. On the other hand, the collisional contribution to the observable $A$ is non-zero now because in this case it includes the collision with the wall.

This method can be applied to the stress tensor as well as to the heat flux. As an example, consider the kinetic part of the heat flux (energy flux density) $\boldsymbol{q}^{\text {kin }}$ infinitely close to the wall but excluding the wall - this means

$$
\boldsymbol{q}^{\text {kin }}=\frac{m / 2}{\tau L_{y}} \sum_{\text {colls }}\left(|\boldsymbol{v}|^{2}\left(\begin{array}{c}
\operatorname{sgn}\left(v_{x}\right) \\
\frac{v_{y}}{\left|v_{x}\right|}
\end{array}\right)+\left|\boldsymbol{v}^{\prime}\right|^{2}\left(\begin{array}{c}
\operatorname{sgn}\left(v_{x}^{\prime}\right) \\
v_{y}^{\prime} \\
\left|v_{x}^{\prime}\right|
\end{array}\right)\right),
$$

where the sum runs over all collisions between a particle and the wall in the time interval $\tau$. Including the wall in the box yields an additional factor of $1 / 2$ as discussed above.

For smooth walls (as used in the simulations), $v_{y}^{\prime}=v_{y}$. This simplifies the kinetic contribution to the heat flux exactly at the wall (i.e. including the wall) to

$$
\boldsymbol{q}^{\mathrm{kin}}\left(\mp L_{x} / 2\right)=\frac{1}{2} \frac{1}{\tau L_{y}} \frac{m}{2} \sum_{\text {colls }}\left(\begin{array}{c} 
\pm\left(v_{x}^{\prime 2}-v_{x}^{2}\right) \\
\left(\left|v_{x}^{\prime}\right|+\left|v_{x}\right|\right) v_{y}
\end{array}\right) .
$$

The post-collisional velocities (primed) are given by the collision rule, Eq. (2.17). For longtime averages the $y$-component of the heat flux is expected to average to zero which is confirmed by our simulations. The $x$-component of the kinetic part of the heat flux at the wall turns out to be identical to the collisional part, Eq. (5.9). If we take an infinitissimal step inside the cell then the kinetic part will be twice this value and the collisional term will vanish, yielding the same total heat flux at the wall, as expected. 


\section{Hydrodynamic Fields in Simulations of Vibrated Systems}

In this chapter we discuss hydrodynamic fields from simulations of vibrated systems of smooth particles, as computed from Eqs. (4.11) - (4.16) and (4.26), (4.38), (5.3), (5.4), and (5.5). Due to the absence of a local velocity field $\boldsymbol{V}$, these equations simplify accordingly. We have performed simulations for a wide range of system parameters $\alpha, N, L_{x}$, and $L_{y}$, and present examples thereof below. Comparing systems with various inelasticities we face the problem of stationary temperatures that are orders of magnitude apart. To have all these systems relax to the same stationary temperature we will introduce an adjusted driving mechanism before discussing hydrodynamic fields in detail.

The following sections show the profile of each hydrodynamic field for four examplary systems, i.e. two different coefficients of restitution at two different densities. After that, the coeffient of restituion will be varied over the full range for two different densities. Finally, we present variations in the hydrodynamic fields with the box edge $L_{x}$, where $L_{x}$ is varied over the full range for two different coefficients of restitution.

Due to the rich behavior of the systems studied, this chapter is primarily a descriptive collection of (selected) data from our simulations. Some relations between the fields will be touched as well but the very interesting tests of constitutive relations will be deferred to chapters 7 and 8 , where we will relate to each other various hydrodynamic fields that are discussed in this chapter on a stand-alone basis. First we will discuss the question of a local equation of state in chapter 7, relating the pressure $p(x)$, density $\rho(x)$, and temperature $T(x)$. In chapter 8 we will check Fourier's law of thermal conductance and its generalization for granular gases which relates the heat flux $q(x)$ to the gradients of the temperature $\frac{d}{d x} T(x)$ and the density $\frac{d}{d x} \rho(x)$.

\subsection{How to Scale the Driving as a Function of the Coefficient of Restitution}

Before we present hydrodynamic fields we show how to drive the system in order to have a well-defined elastic limit. Energy balance, Eq. (3.14) chapter 3, suggested - and Fig. 3.1 confirmed - that the spatially averaged stationary total temperature is approximately proportional 


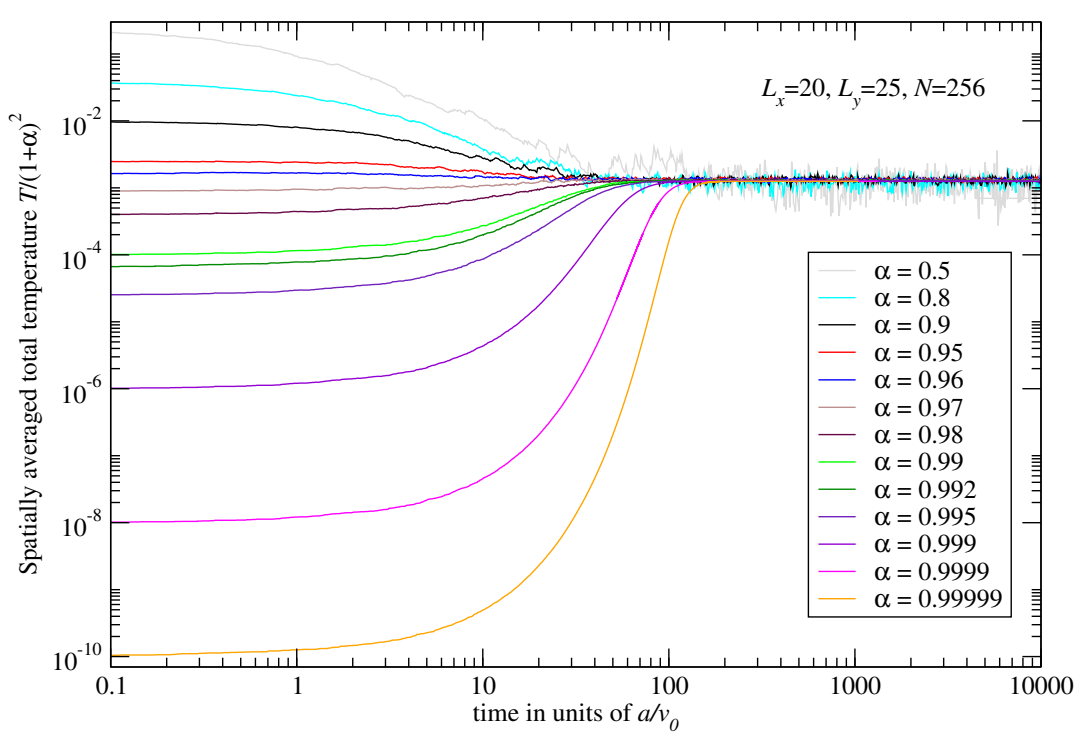

Figure 6.1.: Dynamic time evolution of the spatially averaged total granular temperature $T$ as a function of dimensionless time (i.e. time in units of $\left[a / v_{0}\right]$ ) for systems with the same number of particles $N=$ 256 and the same geometry ( $L_{x}=20, L_{y}=25$ ) but various coefficients of restitution, $0.5 \leq \alpha \leq$ 0.99999. Depending on the coefficient of restitution each system is driven with its peculiar driving velocity $\varepsilon \equiv 1-\alpha^{2}$ and started with an initial temperature $T_{0}=\varepsilon^{2}$. Shown are the temperatures divided by $(1+\alpha)^{2}$ to improve the data collapse for higher inelasticities $(\alpha<0.9)$.

to $\varepsilon^{-2}$ with $\varepsilon \equiv 1-\alpha^{2}$ for $\alpha$ close to unity. Therefore, we must drive a system of particles with a given coefficient of restitution $\alpha$ with a driving velocity

$$
v_{\mathrm{dr}}=\varepsilon=1-\alpha^{2}
$$

in dimensionless units to have systems with different coefficients of restitution relax to approximately the same final temperature. In other words, we drive the system in this chapter with a physical driving velocity $v_{\mathrm{dr}}=\varepsilon v_{0}$ and measure velocities in units of $v_{0}$, i.e. times in units of $a / v_{0}$.

Simulations confirm the suggested driving: Fig. 6.1 shows the relaxation of the granular temperatures as a function of time. For systems with the same number of particles $N=256$ and identical geometry $\left(L_{x}=20\right.$ and $\left.L_{y}=25\right)$ we vary the coefficient of restitution $0.5 \leq \alpha \leq 0.99999$. Each system is driven according to Eq. (6.1) and started with an initial temperature $T_{0}=\varepsilon^{2}$. All these systems relax to approximately the same final temperature $T$. Especially for systems that are not too inelastic the agreement is very good. The relaxation time for reaching the steady state becomes longer the more elastic the systems are. In the perfectly elastic case (with zero driving) the relaxation time is infinite since any initial temperature will prevail forever. To improve the data collapse for moderate to high inelasticities the measured temperature is divided by $(1+\alpha)^{2}$. This makes no difference for $\alpha$ close to one. The 
origin of this additional factor is unclear to us but the same factor is found to also improve the data collapse of the components of the stress tensor and the heat flux.

\section{Deduced Dependencies on the Coefficient of Restitution}

Dimensional analysis shows that the density is proportional to $v_{\mathrm{dr}}^{0}$, temperatures and stresses are proportional to $v_{\mathrm{dr}}^{2}$ while the heat flux is proportional to $v_{\mathrm{dr}}^{3}$. Combined with the energy balance argument, Eq. (3.14), this leads to a mean stationary temperature and mean stationary stresses that are approximately independent of the coefficient of restitution for quasi-elastic systems. The global density is, of course, also independent of the coefficient of restitution. Since a freely evolving system of perfectly elastic particles between two elastic walls (i.e. the limit $\varepsilon \rightarrow 0$ ) has zero heat flux and zero local energy loss, we expect those fields to vanish with $\varepsilon$ for $\varepsilon \rightarrow 0$. The same argument holds for the gradients of the density and temperature. Additionally, the spatial variation ("profiles") of all hydrodynamic fields are expected to depend on $\varepsilon$, of course. This is confirmed by the simulations shown later in this chapter.

\subsection{Density and Temperature Profiles}

We first present data for the density and temperature to demonstrate that the systems considered are strongly inhomogeneous even for collisions with $\alpha=0.9$ and moderately low densities. These and all other hydrodynamic fields will be shown for four exemplary systems, two different densities at two different coefficients of restitution: Systems (a) and (b) use $L_{x}=20$, $L_{y}=25, N=256$ corresponding to $\phi_{0} \equiv a^{2} N /\left(4 L_{x} L_{y}\right)=0.4$ and $\lambda=10.24$, while systems (c) and (d) use $L_{x}=50, L_{y}=25, N=240$ corresponding to $\phi_{0}=0.15$ and $\lambda=9.6$. The coefficient of restitution is $\alpha=0.9$ for systems (a) and (c) while it $\alpha=0.5$ has been used for systems (b) and (d).

In the main graphs of Figs. 6.2 we show the local area fraction $\phi(x)$ (full green lines), the $x$ and $y$-component of the granular temperature, $T_{x}(x)$ and $T_{y}(x)$ (dashed blue and dotted red lines), as well as the isotropic or total temperature $T(x)=\left[T_{x}(x)+T_{y}(x)\right] / 2$ (dashed-dotted indigo lines) for four different sets of parameters on a semi-logarithmic scale. The particle size is indicated by the circles in the corners of the graphs and the inset shows the density profiles on a linear scale. Fig. 6.2 (a) is typical for systems with quite elastic collisions $(\alpha=0.9)$ and moderately high densities $\left(\phi_{0}=0.4\right)$, whereas Fig. 6.2 (b) shows the same system with higher inelasticity $\alpha=0.5$. Figs. 6.2 (c) and (d) show rather dilute systems $\left(\phi_{0}=0.15\right)$, again at two different inelasticities, a moderate one $(\alpha=0.9)$ and a strong one $(\alpha=0.5)$. We note that all quantities are symmetric in $x$ as expected. Except for a small area of approximately one disk diameter next to the walls (indicated by the vertical lines), the area fraction is a monotonic function for either half of the system with the maximum value in the middle of the system. The 

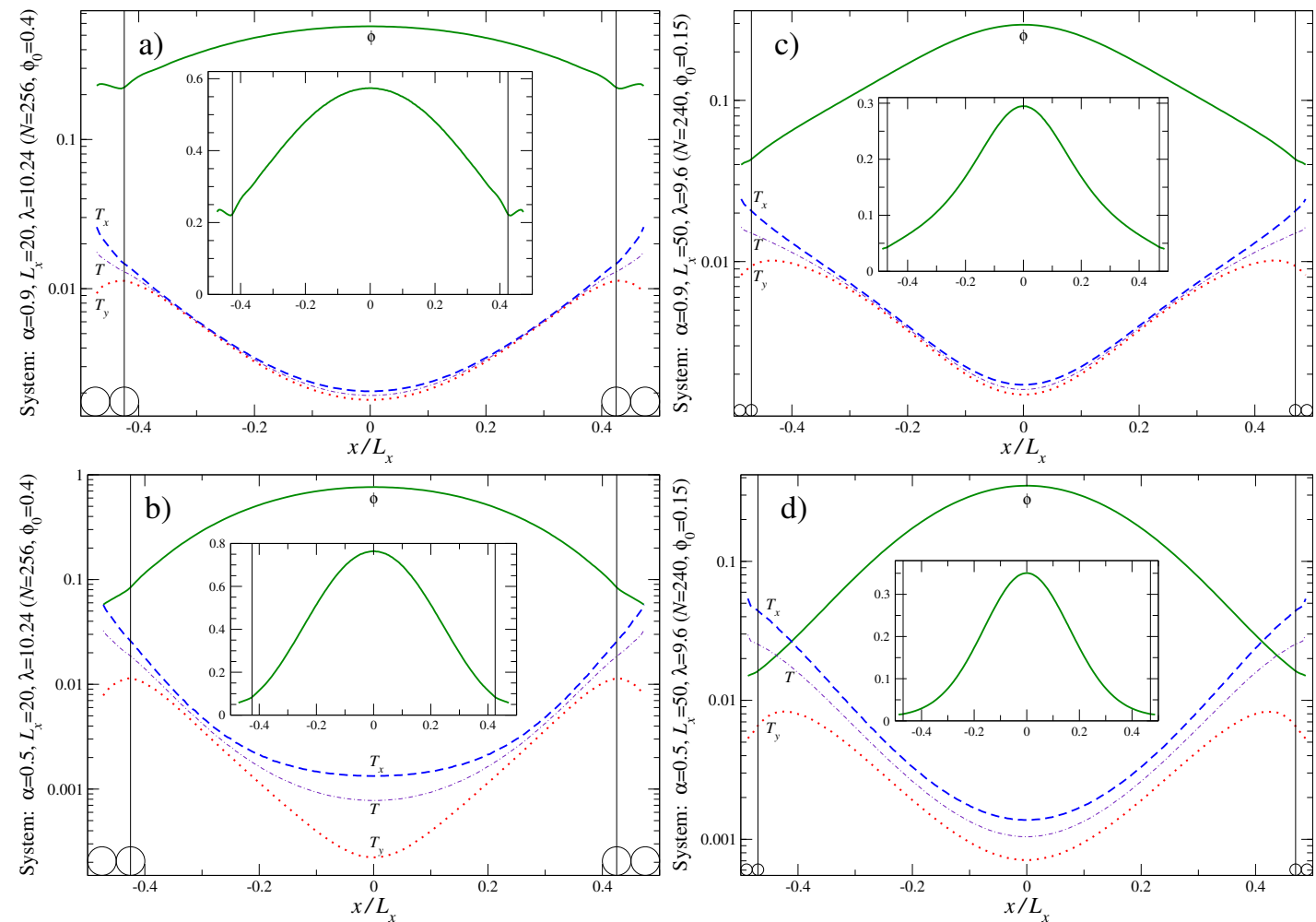

Figure 6.2.: Spatial profiles of the area fraction $\phi$ (full green lines in the main graphs (semi-logarithmic scale) and in the insets on a linear scale) and granular temperatures $T_{x}$ (dashed blue lines), $T_{y}$ (dotted red lines), and $T$ (dash-dotted indigo lines) from simulations for 4 typical systems on a semi-logarithmic scale: (a) $\alpha=0.9, L_{x}=20, \lambda=10.24, N=256, \phi_{0}=0.4$, corresponding to a mean free path $\ell / L_{x} \approx 0.023$, (b) same as (a) but for $\alpha=0.5$, (c) $\alpha=0.9, L_{x}=50, \lambda=9.6, N=240, \phi_{0}=0.15$, corresponding to a mean free path $\ell / L_{x} \approx 0.033$, (d) same as (c) but for $\alpha=0.5$. The insets show the profile of the area fraction on a linear scale. Even for moderately low densities and inelasticities the systems are highly inhomogeneous.

temperatures $T_{x}, T_{y}$, and $T$ are monotonic as well with the lowest temperatures in the middle of the sample.

The reason for the increased density next to the wall in Fig. 6.2 (a) is an effective attractive potential of the wall due to entropic effects: Once a disk gets closer to the wall than one disk diameter, it can only receive hits from within the box but no hits from the direction of the wall. Thus the particle is pushed closer to the wall [RY84, HM86]. This effect is partially compensated by the driving walls, which add momentum to any particle hitting the walls. To support this explanation we have also investigated systems of half the size $-0.5 \leq x / L_{x} \leq 0$, half the number of particles and with an elastic wall at $x=0$. The resulting hydrodynamic fields (not shown) are almost identical to the ones in Figs. 6.2 except for a small region of about one diameter close to $x=0$ where the aforementioned effect is particularly visible. 

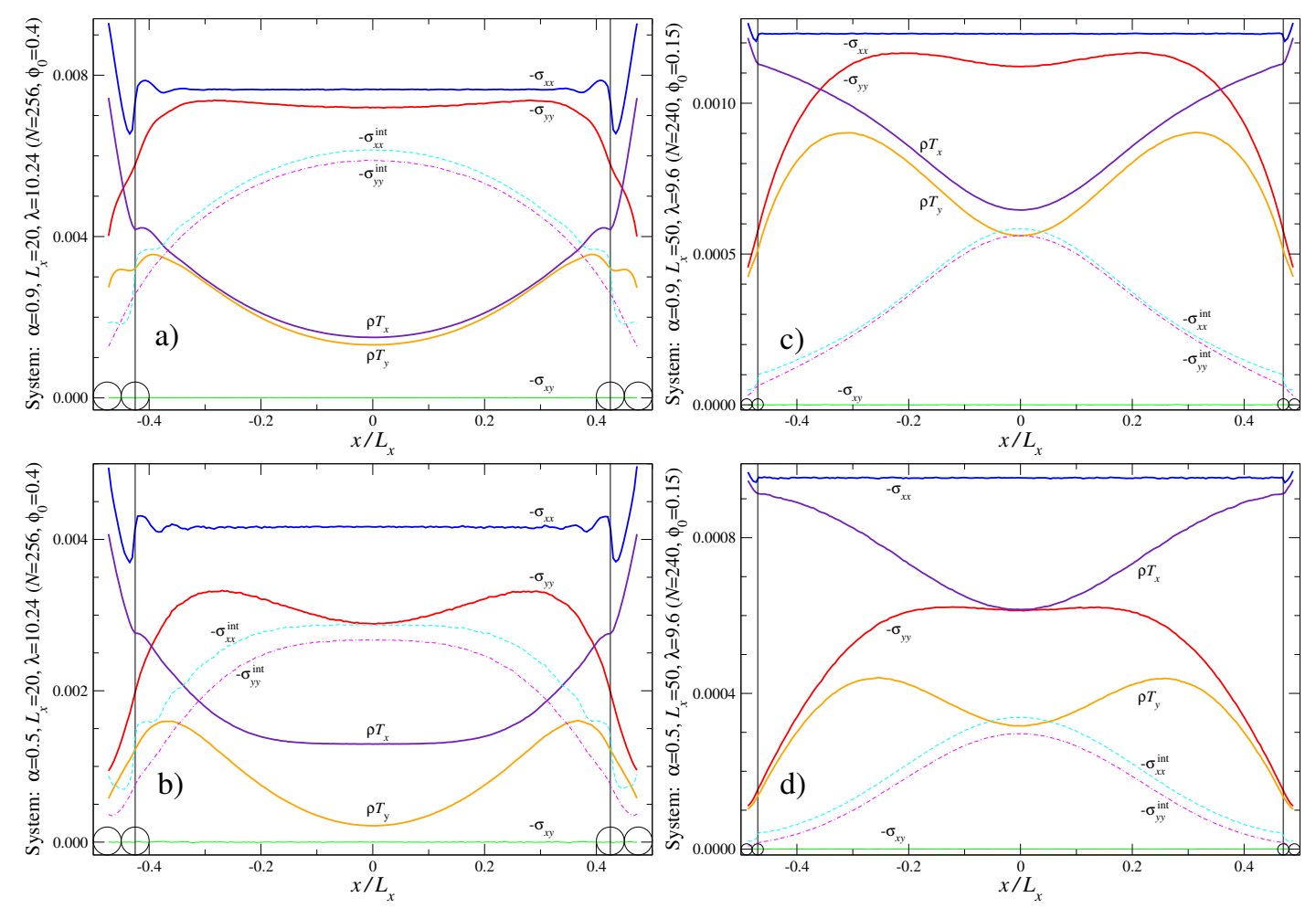

Figure 6.3.: Spatial profiles of the stress tensor components $-\sigma_{x x}$ (blue lines), $-\sigma_{y y}$ (red lines), $-\sigma_{x y}$ (green lines), their kinetic parts $\rho T_{x} \equiv-\sigma_{x x}^{\text {kin }}$ (indigo lines) and $\rho T_{y} \equiv-\sigma_{y y}^{\text {kin }}$ (orange lines), as well as their collisional contributions $-\sigma_{x x}^{\text {int }}$ (cyan lines) and $-\sigma_{y y}^{\text {int }}$ (magenta lines) for the same systems as in Figs. 6.2. Note that the $x x$-component is constant throughout the system for all parameters (except for a marginal region of about one particle diameter) while the $y y$-component displays a dip in the middle of the sample. For both components the collisional part of the stress tensor is important and may not be neglected.

In systems exhibiting so called stripe states [LMS02a, LMS02b], which are homogeneous in $y$, but have an enhanced density in the middle of the sample, we have sometimes observed an oscillatory instability of a central dense cluster in the $x$-direction [KM04], in particular if $\alpha$ is low and $L_{x}$ is large. However, these oscillations occur on far shorter time scales compared to the time interval $\tau$, over which we average to obtain stationary-state quantities. Hence, these oscillations are completely averaged out in the data presented.

\subsection{Stress Tensor and Pressure}

For an isotropic system the $x x$ - and the $y y$-component of the stress tensor would be equal and the negative of the pressure. We therefore define the local pressure $p(x):=-\left[\sigma_{x x}(x)+\right.$ $\left.\sigma_{y y}(x)\right] / 2$ and always show the the negative of the components of the stress tensor. In Figs. 6.3 
we show the components of the negative stress tensor $-\sigma_{x x}(x),-\sigma_{y y}(x)$, and $-\sigma_{x y}(x)$ as well as their kinetic and collisional contributions for the same four different sets of parameters as shown in Figs. 6.2. The off-diagonal component $-\sigma_{x y}$ of the stress tensor is always vanishingly small. The $x x$-component, $-\sigma_{x x}$, is constant within the sample except for a boundary layer close to the driving walls. $-\sigma_{y y}$ displays a dip in the center of the sample, which is more pronounced for the more dilute and more elastic system in Fig. 6.3 (c) as well as in the denser and more inelastic system in Fig. 6.3 (b). It is hardly visible in the strongly inelastic, dilute system of Fig. 6.3 (d). Furthermore, $-\sigma_{y y}$ increases considerably over a broad range in the more dilute systems in Figs. 6.3 (c) and (d) when moving from a driving wall towards the center of the system. Currently, the origin of both $x$-dependences of $-\sigma_{y y}$ is not clear to us even though it seems that the dip stems from the kinetic contribution through the anisotropy of the granular temperatures $\left(T_{x} \neq T_{y}\right)$. While the stationary-state condition $\boldsymbol{\nabla} \cdot \boldsymbol{\sigma}=0$ and homogeneity in $y$ require $\sigma_{x x}$ and $\sigma_{x y}$ to be constant in $x$ on general grounds, cf. Eq. (4.1), this is not the case for $\sigma_{y y}$. We have carefully checked that the $x$-dependence of $\sigma_{y y}$ is not caused by a shear instability associated with a non-zero velocity field $\boldsymbol{V}(x)$ in the system. Thus, there are normal stresses present in the simulated systems, and they depend on $x$.

If the equation of state of the ideal gas held, the local pressure $p(x)$ would be related to the local temperature and density according to $p(x)=\rho(x) T(x)=(4 / \pi) \phi(x) T(x)$. For our systems the kinetic part of the stress tensor is diagonal and each component is simply related to the corresponding temperatures $-\sigma_{x x}^{\mathrm{kin}}(x)=\rho(x) T_{x}(x)$ and $-\sigma_{y y}^{\mathrm{kin}}(x)=\rho(x) T_{y}(x)$. Hence the difference between the negative of the measured stress tensor and the ideal gas behavior is due to collisions. From Figs. 6.3 it can be seen that the stress tensor deviates strongly from the ideal gas behavior. Consequently, collisions contribute significantly. Here the collisional part has been measured directly in the simulations and not only estimated by approximate theories as has been done elsewhere [BRMM00, BT02b].

We have also estimated the global mean free path $\ell$ for our simulated systems according to Eq. (9) in Ref. [GZBN97] which expresses $\ell$ solely as a function of the global area fraction $\phi_{0}$. In our notation it reads $\ell \approx=\frac{\sqrt{2}}{\pi \phi_{0}} \frac{1-\phi_{0} / \phi_{c}}{\left.1-(1-\sqrt{3 / 8}) \phi_{0} / \phi_{c}\right)}$, where $\phi_{c}=\pi /[2 \sqrt{3}] \approx 0.9069$. For the denser systems with $\phi_{0}=0.4$ shown in graphs (a) and (b) of Figs. $6.2-6.5$ as well as in the left graphs of Figs. $6.6-6.11$ this yields $\ell \approx 0.46$ (in units of the diameter of the disks, that is $\left.\ell / L_{x} \approx 0.023\right)$. For the thinner systems with $\phi_{0}=0.15$, shown in graphs (c) and (d) of Figs. $6.2-6.5$, one gets $\ell \approx 1.64$, that is $\ell / L_{x} \approx 0.033$. For the systems studied in the right graphs of Figs. $6.6-6.11$ we get $\ell \approx 2.56$, that is $\ell / L_{x} \approx 0.032$. Thus, in all cases the mean free path $\ell$ is much smaller than the scales governing the spatial variations of the hydrodynamic fields, which are of order $L_{x}$. 

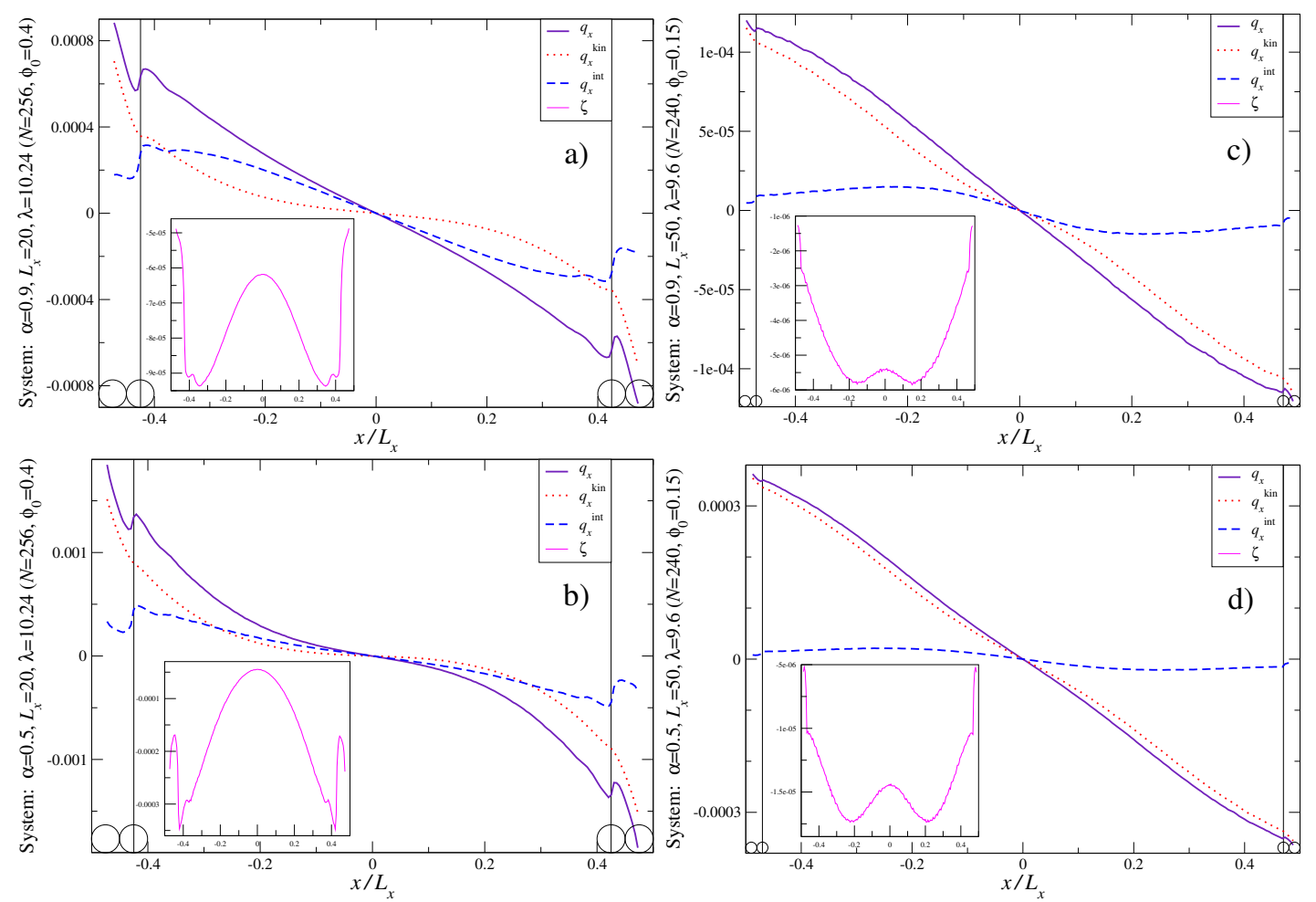

Figure 6.4.: Spatial profiles of the $x$-component of the heat flux (main graphs) and the local energy loss (insets) for the same systems as in Figs. 6.2. The full indigo lines show the heat flux $q_{x}$ while the dotted red lines represent the kinetic and the dashed blue lines the collisional contribution. Clearly, the collisional heat transfer is an important contribution to the heat flux. Depending on the system parameters the highest dissipation, i.e. the maximum of the absolute value of the local energy loss (i.e. the minimum of $\zeta$ ) takes place somewhere between the walls and the middle of the sample.

\subsection{Heat Flux}

In the main graphs of Figs. 6.4 we show the $x$-component of the heat flux $q_{x}$ as a function of the normalized position $x / L_{x}$ as well as its kinetic and collisional contribution for the same four systems as displayed in Figs. 6.2 and 6.3. The $y$-component vanishes as expected. The $x$-component of the heat flux is anti-symmetric about the middle of the sample and linear to zeroth approximation. It is directed towards the center of the system at all positions. This is not surprising since everywhere in the sample net energy flows from the walls towards the center where part of the energy is being dissipated while the remaining part flows further towards the center. In the middle of the system the net flow must vanish for symmetry reasons. In contrast, a system of perfectly elastic particles driven by a vibrating wall on one side and dissipating energy through collisions with an inelastic wall on the other side is expected to yield a linear temperature gradient and, consequently, a constant heat flux. This has been confirmed by simulations (not shown). 


\subsection{Local Energy Loss}

In a $y$-independent stationary state with zero mean velocity at all positions, as we expect and find it in our simulations, Eq. (4.36) can be integrated to yield

$$
q_{x}\left(-L_{x} / 2\right)-q_{x}\left(+L_{x} / 2\right)=\int_{-L_{x} / 2}^{L_{x} / 2} \zeta(x) d x
$$

This is confirmed by our simulations: In the stationary state the energy input at the walls is balanced by the energy dissipated due to collisions in the entire system. That means that the energy loss rate $\zeta(x)$ should scale like the heat flux $q_{x}(x)$ divided by $L_{x}$. Of course, the shape of the profile is different, in general.

In the insets of Figs. 6.4 (a) - (d) we show the local energy loss profile $\zeta$ as a function of the normalized position $x / L_{x}$ for the four systems already considered in Figs. 6.2, 6.3, and the main graph of 6.4. We see that in all those cases the maximum energy loss $\left(\max _{x}|\zeta(x)|\right)$ occurs somewhere between the wall and the middle of the system, at $x \sim \pm 0.3 L_{x}$ for the dense systems and at $x \sim \pm 0.2 L_{x}$ for the dilute systems.

\subsection{Gradients of the Density and Temperature}

Since we carefully designed the simulations to yield very precise high resolution density and temperature profiles that become very smooth for sufficiently long runs it is possible to extract the gradient fields for the density and temperature.

Figs. 6.5 show the density and temperature gradients of the four systems of Figs. 6.2. Since the gradients are much more sensitive to fluctuations than the underlying fields we will later show smoothed gradients in Figs. $6.12-6.13$ and $6.20-6.21$. Here, though, we show the unsmoothed raw data. For better visualization, the temperature gradient has been multiplied by a factor of ten, i.e. we show $10 \frac{d}{d x} T(x)$ (dashed lines) and $\frac{d}{d x} \rho(x)$ (solid lines). The two moderately inelastic systems (a) and (c) reveal temperature gradients that are linear to first approximation. The two strongly inelastic systems (b) and (d) show temperature gradients that are linear in the middle only. To the same order of approximation, the density gradient is linear in system (a) only, which is moderately dense and moderately inelastic. It is shaped like an oblique " $\mathrm{z}$ " in the three other cases. 

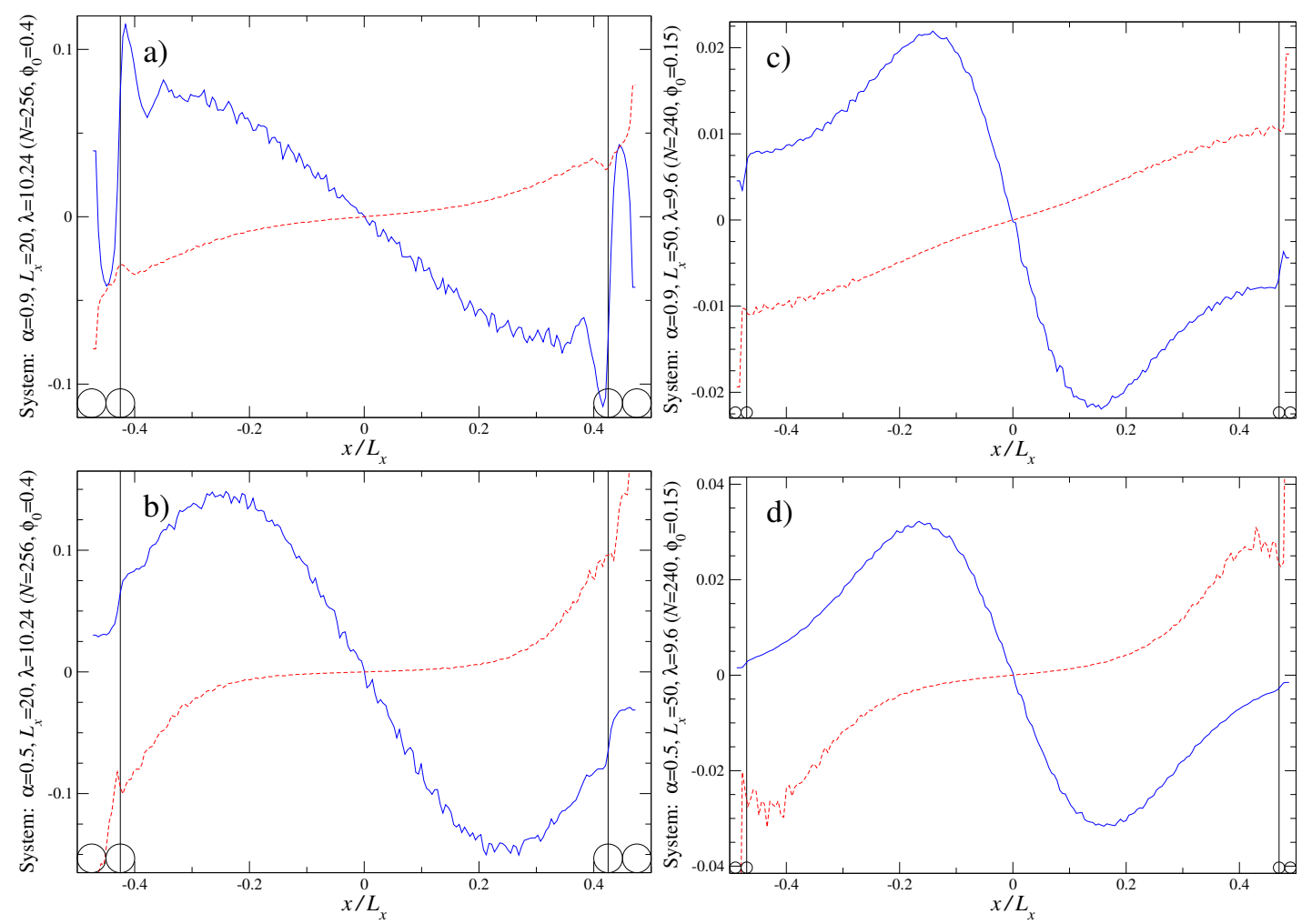

Figure 6.5.: Spatial profiles of the density and temperature gradients for the same four systems as in Figs. 6.2. The full blue lines show the density gradients $\frac{d}{d x} \rho(x)$ while the dashed red lines show the temperature gradients multiplied by 10 for better visualization, $10 \frac{d}{d x} T(x)$.

\subsection{Variation of the Coefficient of Restitution $\alpha$ at Fixed System Sizes}

In this section we compare all hydrodynamic fields for various coefficients of restitution, $0.5 \leq$ $\alpha \leq 0.99999$. In all figures presented the left graph shows simulations with $L_{x}=20, L_{y}=25$, and $N=256$, corresponding to a total area fraction of $\phi_{0}=0.4$, while the right graphs show simulations with $L_{x}=80, L_{y}=25$, and $N=256$, corresponding to a total area fraction of $\phi_{0}=0.1$. Note that the color code is the same for all Figs. 6.6-6.13.

\section{Density and Temperature}

Figs. 6.6 and 6.7 compare the area fraction (density) and temperature profiles for various coefficients of restitution. The temperature profiles have been divided by $(1+\alpha)^{2}$ to improve the data collapse for the more inelastic systems $(\alpha<0.9)$. This enhances the fit significantly for moderate to high inelasticities while making no difference for $\alpha$ close to one. For almost elastic 

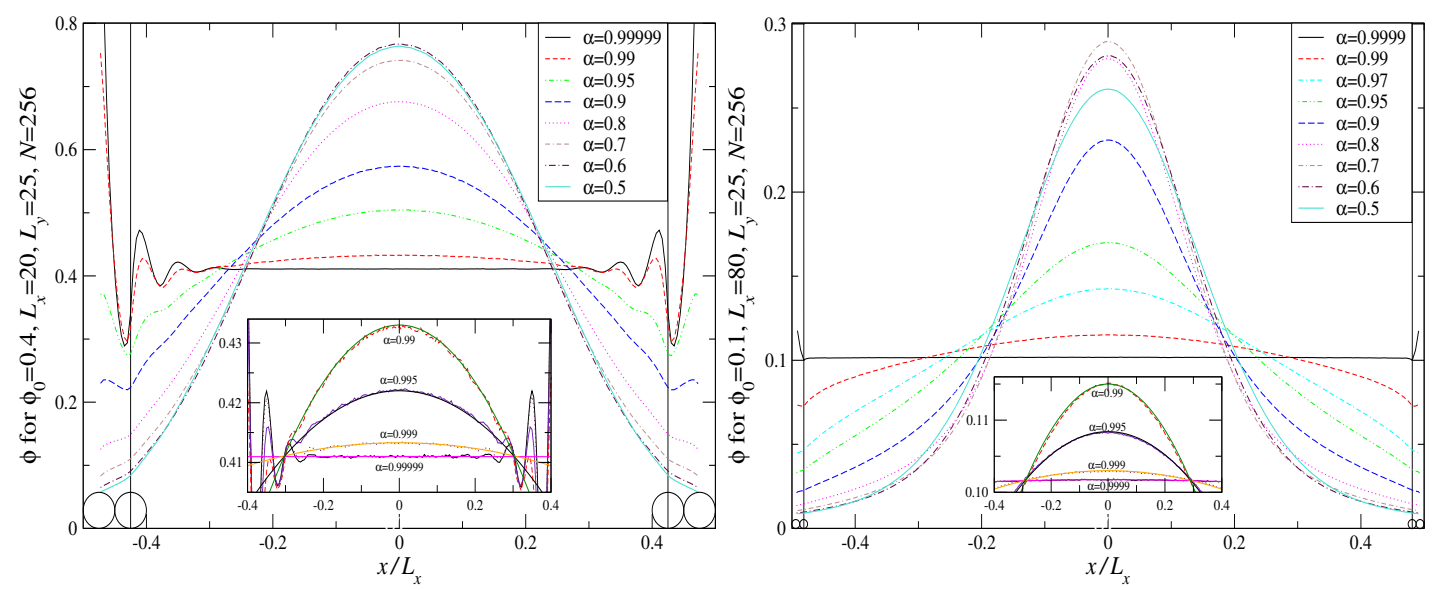

Figure 6.6.: Spatial profiles of the area fraction $\phi(x)$ for a moderately dense and a dilute system for various inelasticities each. The left graph shows the same system size and number of particles presented in Figs. 6.2 (a) for various values of $\alpha$. The right graph displays the same system as the left one except that here $L_{x}=80$ instead of 20, leading to a total area fraction $\phi_{0}=0.1$ instead of $\phi_{0}=0.4$ in left graph. The insets show quasi-elastic systems and a fit discussed in Sec. 6.9.1. Clearly, the profiles become flatter as the systems become more elastic.

systems the profiles are almost flat. With increasing inelasticity the middle of the systems becomes denser and cooler. In addition, the density profile changes its shape from near-quadratic to bell-shaped when changing the inelasticity from quasi-elastic to inelastic. In both graphs the insets show the quasi-elastic runs, $\alpha$ close to one, with additional fits that are discussed in Sec. 6.9.1. Note that the density profiles as well as the temperature profiles show the highest inhomogeneities between $\alpha=0.6$ and $\alpha=0.8$. For higher inelasticities, e.g. $\alpha=0.5$, the profiles flatten out a bit. This may be due to the collective motion of a central dense cluster in the strongly inelastic cases.

\section{Stresses}

Figs. 6.8 and 6.9 compare the $x x$ - and the $y y$-components of the rescaled stress tensor $-\boldsymbol{\sigma} /(1+$ $\alpha)^{2}$ for the same systems as in Figs. 6.6 and 6.7, i.e. for two different densities (left: $\phi_{0}=0.4$, right: $\left.\phi_{0}=0.1\right)$ and various inelasticities $(0.5 \leq \alpha \leq 0.99999)$. We can see clearly that the profiles for the $x x$-component (Figs. 6.8) are similar in shape for all systems. For the dense systems (left graph of Figs. 6.8) they all lie within a region around the quasi-elastic case represented by the black bold line. For the more dilute system (right graph of Figs. 6.8) the mean value of $-\sigma_{x x} /(1+\alpha)^{2}$ decreases monotonically with increasing $\alpha$. Again, the black bold line (the lowest in magnitude) represents the quasi-elastic case $\alpha=0.99999$. 

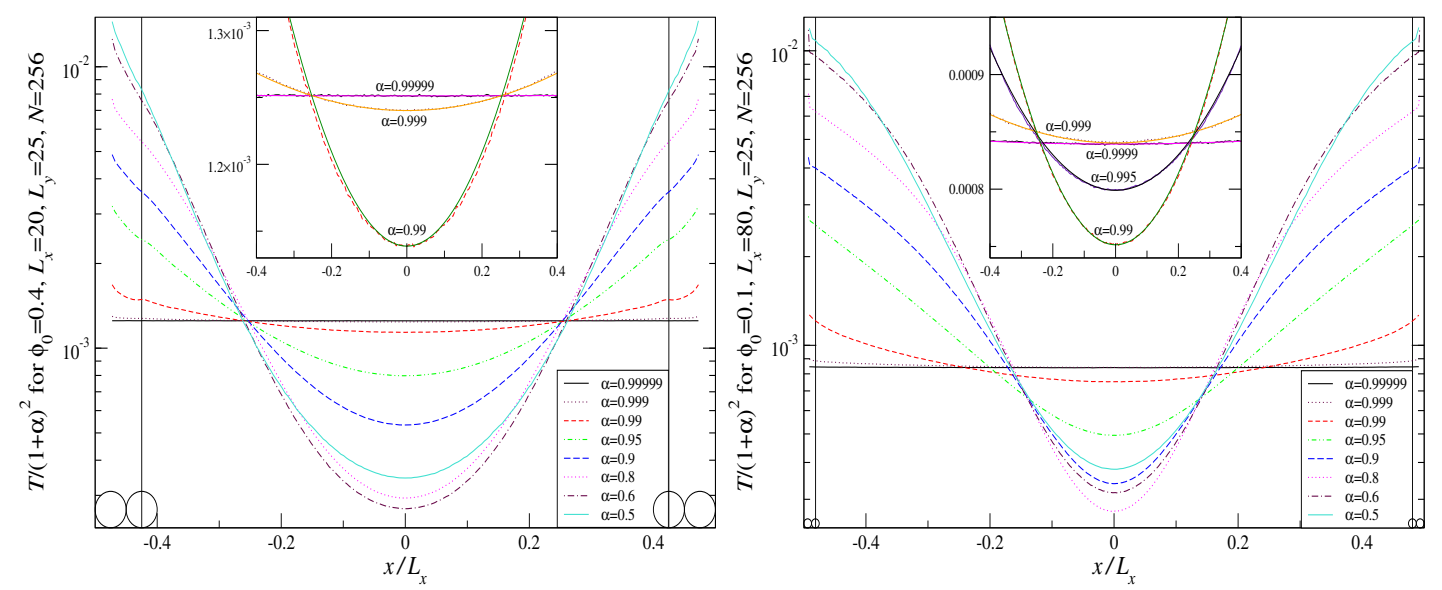

Figure 6.7.: Spatial profiles of the rescaled temperature $T /(1+\alpha)^{2}$ for the same systems as in Figs. 6.6. (Left: $\phi_{0}=0.4$, right: $\phi_{0}=0.1$ ). Again, the insets show quasi-elastic systems and a fit discussed in Sec. 6.9.1, and again the profiles become flatter as the systems become more elastic.

The behavior of the $y y$-component of the rescaled stress tensor $-\sigma_{y y} /(1+\alpha)^{2}$ (Figs. 6.9) is more interesting: While the mean value still lies in a region around the quasi-elastic case (black bold lines) the shape of the profiles depends strongly on the system parameters. Additionally, $-\sigma_{y y}$ reveals a dip in the middle of the sample for all systems. As expected, in the quasielastic case the $y y$-component is very similar to the $x x$-component both in magnitude and in shape (compare Figs. 6.9 with Figs. 6.8), in particular also the $y y$-component is almost constant throughout the sample (black bold lines). Increasing the inelasticity a bit from the quasi-elastic limit (see, for example, the double-dotted-dashed green lines $(\alpha=0.95)$ in Figs. 6.9) causes $-\sigma_{y y}(x) /(1+\alpha)^{2}$ to increase in the middle of the sample and in a wide region around the middle $\left(-0.35 \lesssim x / L_{x} \lesssim 0.35\right)$. Close to the walls $-\sigma_{y y}(x) /(1+\alpha)^{2}$ is significantly reduced. For higher inelasticities the behavior depends on the density. For the higher density systems (left graph of Figs. 6.9) the overall value of the $y y$-component of the rescaled stress tensor decreases for increasing inelasticity while the dip in the middle at $x=0$ becomes more and more pronounced. In the lower density systems (right graph) the behavior is far less monotonic: If the inelasticity is increased a bit (see, for example, the dashed blue line $(\alpha=0.9)$ ), the dip becomes more pronounced while the "peaks" at $x \sim 0.2$ further increase in magnitude. For $\alpha=0.8$ (dotted magenta line) the dip is deepest while the peaks have already come down a bit. For even higher inelasticities the dip flattens out and the overall value of $-\sigma_{y y} /(1+\alpha)^{2}$ decreases again, see $\alpha=0.5$ (cyan full line).

\section{Heat Flux}

Figs. 6.10 show the rescaled heat fluxes $q_{x} /\left(\varepsilon[1+\alpha]^{2}\right)$ for the systems of Figs. 6.6 - 6.9: For two densities (left: $\phi_{0}=0.4$, right: $\left.\phi_{0}=0.1\right)$ and various inelasticities $(0.5 \leq \alpha \leq 0.99999)$ 

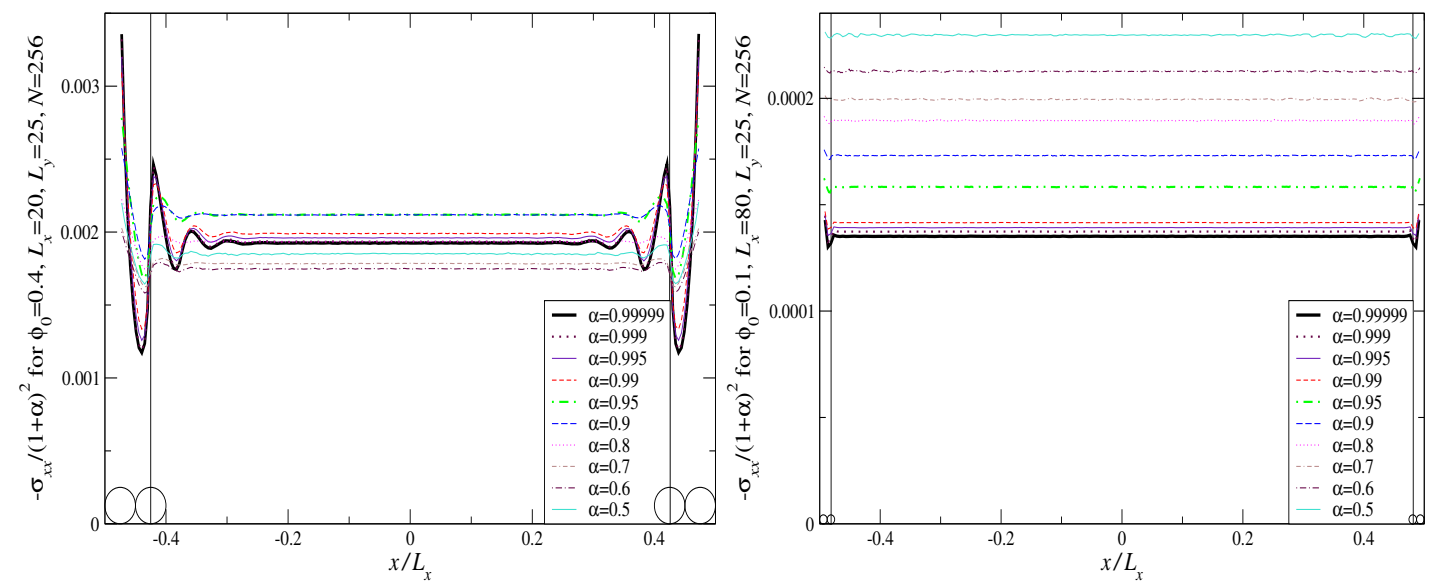

Figure 6.8.: Spatial profiles of the $x x$-component of the rescaled stress tensor $\left(-\sigma_{x x} /(1+\alpha)^{2}\right)$ for the same systems as in Figs. 6.6. (Left: $\phi_{0}=0.4$, right: $\phi_{0}=0.1$ ). While the mean value of the stress is a non-trivial function of the coefficient of restitution and the system size, it is constant (except for a small boundary region) for all systems as expected.
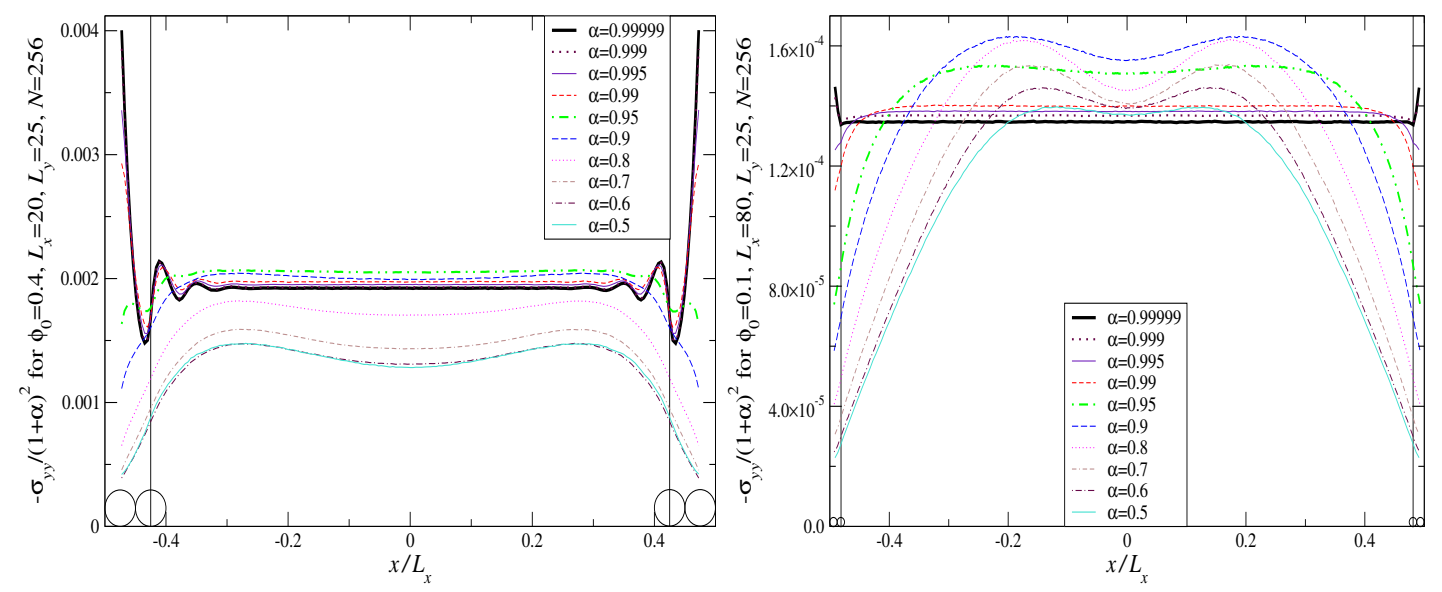

Figure 6.9.: Spatial profiles of the $y y$-component of the rescaled stress tensor $\left(-\sigma_{y y} /(1+\alpha)^{2}\right)$ as in Figs. 6.8 (for the same systems as in Figs. 6.6). (Left: $\phi_{0}=0.4$, right: $\phi_{0}=0.1$ ). Again, the mean value of the stress is a non-trivial function of the coefficient of restitution and the system size. In addition, the shape of the profiles depends on the system parameters and $-\sigma_{y y}$ reveals a dip in the middle for all systems.

we compare the $x$-component of the rescaled heat flux. As described in the beginning of this chapter we expect the heat flux to vanish in the elastic limit. Therefore, we multiply the measured $q_{x}$ by $\varepsilon^{-1}$ to confirm this hypothesis. In Figs. 6.10 we plot $q_{x} /\left(\varepsilon[1+\alpha]^{2}\right)$ and find that all profiles collapse approximately, at least for small inelasticities. This confirms the hypotheses just described. Note that the heat flux profiles are very close to linear for the quasi-elastic cases while they become curved for higher inelasticities. 

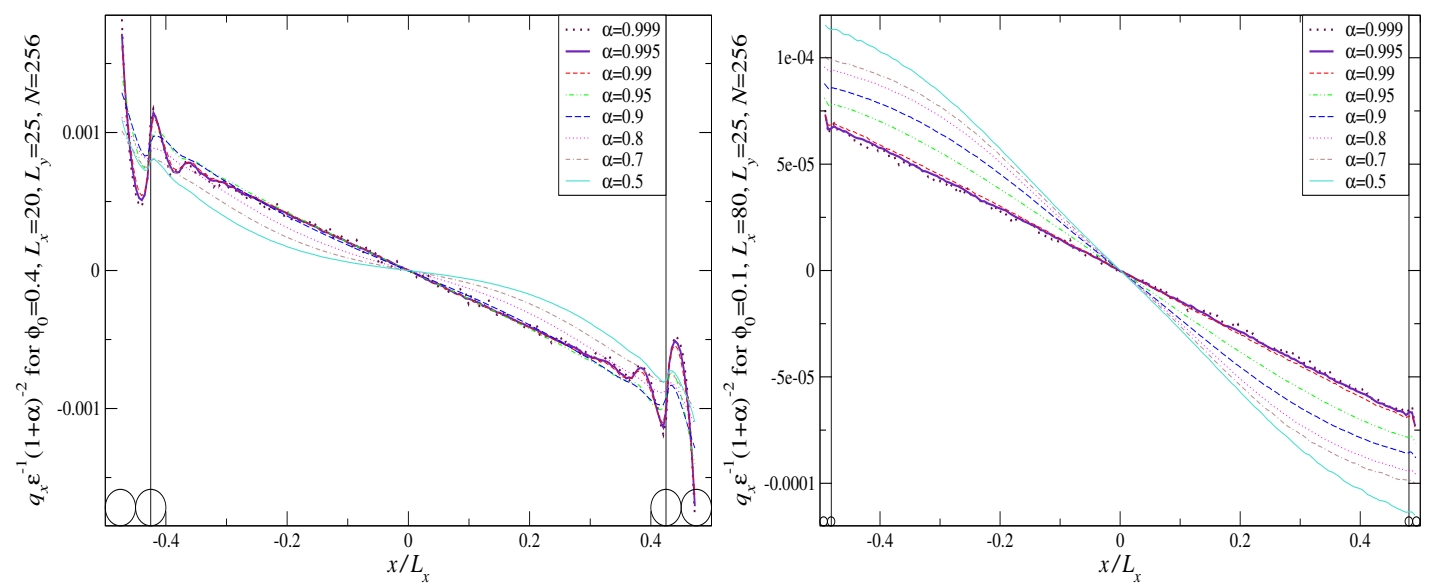

Figure 6.10.: Spatial profile of the rescaled heat flux $q_{x} /\left(\varepsilon[1+\alpha]^{2}\right)$ for the same systems as in Figs. 6.6. (Left: $\phi_{0}=0.4$, right: $\phi_{0}=0.1$ ). Clearly, the scaling suggested captures the correct order of magnitude while the shape of the profile collapses onto one (linear) master curve in the quasi-elastic limit only.

\section{Local Energy Loss}

In Figs. 6.11 we compare the local energy loss for various inelasticities $(0.5 \leq \alpha \leq 0.99999)$ and two different area fractions (left: $\phi_{0}=0.4$, right: $\phi_{0}=0.1$ ), i.e. the same systems as in Figs. 6.6-6.10. The top row shows $\zeta /\left(\varepsilon[1+\alpha]^{2}\right)$ as a function of $x / L_{x}$. We see that for very dilute and/or quasi-elastic systems the dissipation is highest in the middle of the sample while for higher densities and/or higher inelasticities the dissipation (i.e. the absolute value of $\zeta$ ) is highest somewhere between a wall and the middle, at $x \sim \pm 0.3 L_{x}$ for the denser systems and at $x \sim \pm 0.15 L_{x}$ for the dilute systems. The bottom row compares the local energy loss to an estimate by the following simple argument: One would expect the local energy loss to be proportional to the local collision frequency per area - approximately given by Enskog's collision frequency per area $\propto \rho^{2} \chi \sqrt{T}$, cf. Eq. (3.5) - times the average change of energy per collision, approximately given by $-\varepsilon T / 2$, cf. Eq. (3.4). According to Eq. (C.83) and the arguments given in appendix C.6 a correct averaging procedure would yield another factor of 2. Therefore, we expect the energy loss at position $x$ to be

$$
\zeta_{\text {est }}(x):=-\sqrt{\pi} \varepsilon \rho^{2}(x) \chi(x) T^{3 / 2}(x)
$$

or $\zeta / \zeta_{\text {est }}$ to be 1 throughout the system. Here, $\chi(x)$ denotes the Henderson [Hen75] approximation for the pair correlation at contact, Eq. (3.6), evaluated locally using $\phi=\phi(x)$. Eq. (6.3) is in agreement with Ref. [JR85]. Clearly, for the quasi-elastic systems this argument is very good while strong deviations are observed for moderate to high inelasticities. 

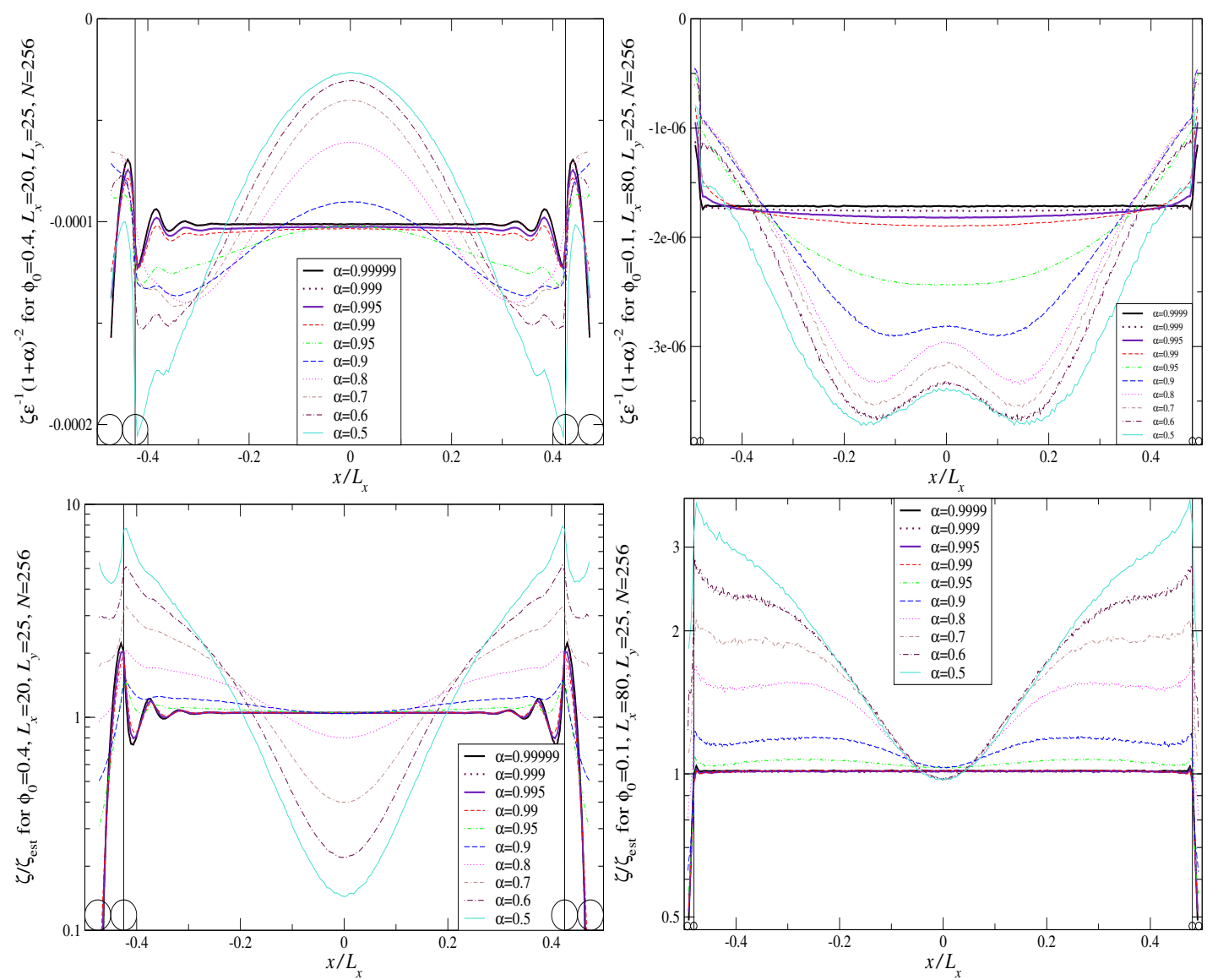

Figure 6.11.: Spatial profiles of the rescaled local energy loss for the same systems as in Figs. 6.6. (Left: $\phi_{0}=0.4$, right: $\phi_{0}=0.1$ ). The top row shows $\zeta /\left(\varepsilon[1+\alpha]^{2}\right)$ with $\zeta$ as given in Eqs. (4.32) and/or (5.5). The bottom row shows $\zeta / \zeta_{\text {est }}$ with $\zeta_{\text {est }}$ as given in Eq. (6.3). $\zeta_{\text {est }}$ estimates the local energy loss by multiplying the theoretical values of the local collision frequency and the average energy lost per collision at that position in the sample. For quasi-elastic systems the estimate is very good. For higher inelasticities the deviations become stronger: For moderately high densities the dissipation close to the walls is higher than estimated by the this simple argument. However, in the middle of the sample the dissipation is lower. For lower densities the dissipation is higher throughout the sample except for a very small region in the middle.

\section{Gradients}

For the systems considered here $\left(v_{\mathrm{dr}}=\varepsilon\right)$ we expect the gradients to vanish for $\varepsilon \rightarrow 0$ because there are no gradients in the limit of elastic particles in a box with elastic walls. As an expansion to lowest order in $\varepsilon$ we therefore expect the gradients to be proportional to $\varepsilon$. Since both the density and temperature profiles are symmetric around $x / L_{x}=0$ we expect them to be proportional to const $\mp\left(x / L_{x}\right)^{2}$ to lowest order in $x$. 

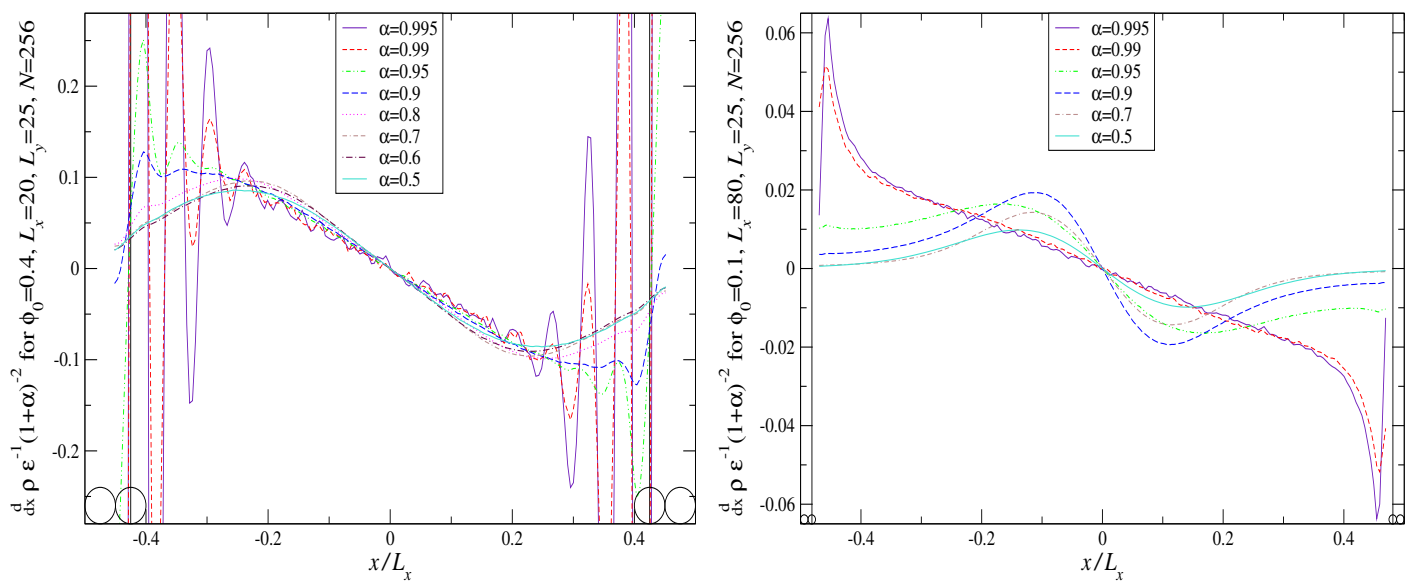

Figure 6.12.: Spatial profile of the density gradient for the same systems as in Figs. 6.6. For the higher density systems (left graph) we observe strong fluctuations of the density gradient for quasi-elastic systems close to the walls. In the middle of the systems the data collapse is very good. For lower density systems the data collapse works only for systems that are not too inelastic $(\alpha \gtrsim 0.99)$. For higher inelasticities the density gradient becomes non-monotonic.
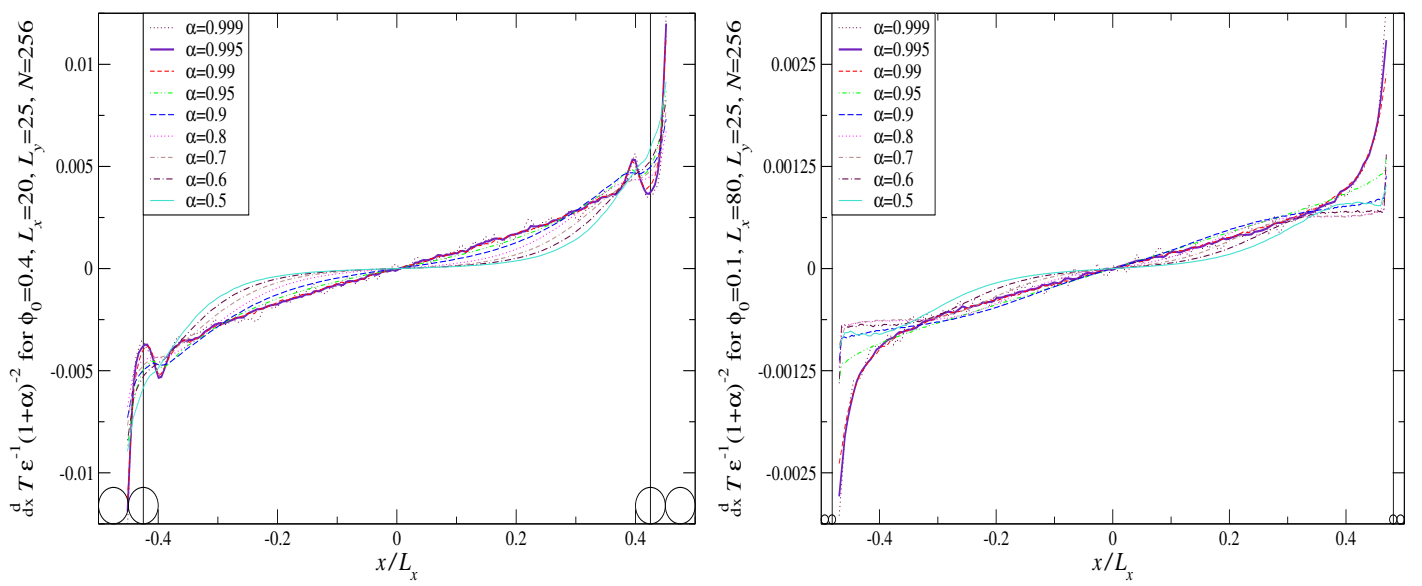

Figure 6.13.: Spatial profile of the temperature gradient for the same systems as in Figs. 6.6. Compared to the density gradients the temperature gradients are much more similar in shape for all systems and the data collapse works better for all systems. Again, a very good data collapse is observed for $\alpha \gtrsim 0.99$.

In Figs. 6.12 and 6.13 we show the smoothed density and temperature gradients both divided by $\varepsilon$ for the systems presented in Figs. 6.6, i.e. as a function of $\alpha$ for two different densities. Again, we observe approximate scaling in the elastic limit where the profiles become linear. For the more inelastic systems the gradients become curved. 


\subsection{Variation of the Density for Fixed Coefficients of Restitution}

In this section we show all hydrodynamic fields for various sizes of the box edge $20 \leq L_{x} \leq$ 8000 (i.e. for various area fractions $0.001 \leq \phi_{0} \leq 0.4$ at fixed line density $N / L_{y}=10.24$ ) for two fixed values of the restitution coefficient $\alpha=0.9$ (left graphs) and $\alpha=0.99$ (right graphs). Again, we use $N=256$ and $L_{y}=25$. Note that the color code is the same again for all Figs. $6.14-6.21$.

\section{Density and Temperature}

In Figs. 6.14 and 6.15 we show the rescaled density and temperature profiles, $\phi(x) L_{x}$ and $T(x)$ respectively, for various sizes of the box edge $L_{x}$ corresponding to various area fractions. Clearly, the density inhomogeneities become more pronounced as the total density decreases. For example, the long-dashed red lines in Figs. 6.14 representing an area fraction of $\phi_{0}=0.4$ are flatter than the ones for $\phi_{0}=0.2$ (dotted blue lines). For very dilute systems all density profiles can be represented by a master curve, cf. also Sec. 6.9.2. For example, the dashed bold black lines representing $L_{x}=8000$, corresponding to an area fraction of $\phi_{0}=0.001$ are hardly distinguishable from the curves for $L_{x}=400$ ( $\phi_{0}=0.02$, full magenta lines). For moderately high area fractions we observe overall concave density profiles (see, for example, the profile for $\phi_{0}=0.4$ depicted by the long-dashed red line in the left graph of Figs. 6.14). For low area fractions the peak increases and for quasi-elastic systems the shape of the profile stays concave. For moderate to high inelasticities the shape of the profile changes and we find a bell-shaped master curve (dashed black line). Cf. also Figs. 6.22 .

Such a master curve is also found for the temperature profiles (Figs. 6.15) but the change in the flattening of the profile for higher densities is much weaker. On the other hand the overall value of the temperature depends more strongly on $L_{x}$ for more elastic systems, e.g. the mean temperature for $L_{x}=20$ is approximately $60 \%$ larger than the one for extremely dilute systems.

\section{Stresses}

In Figs. 6.16 and 6.17 we compare the components of the stress tensor (multiplied by $L_{x}$ as implied by the scaling of the density and temperature) for two fixed coefficients of restitution, $\alpha=0.9$ (left graphs) and $\alpha=0.99$ (right graphs). Again, we observe scaling for the dilute limits. The shape of the profiles does not change very much for $-\sigma_{x x}(x)$. Its mean value increases with increasing density. For $-\sigma_{y y}(x)$ we observe a considerable change in the shape 

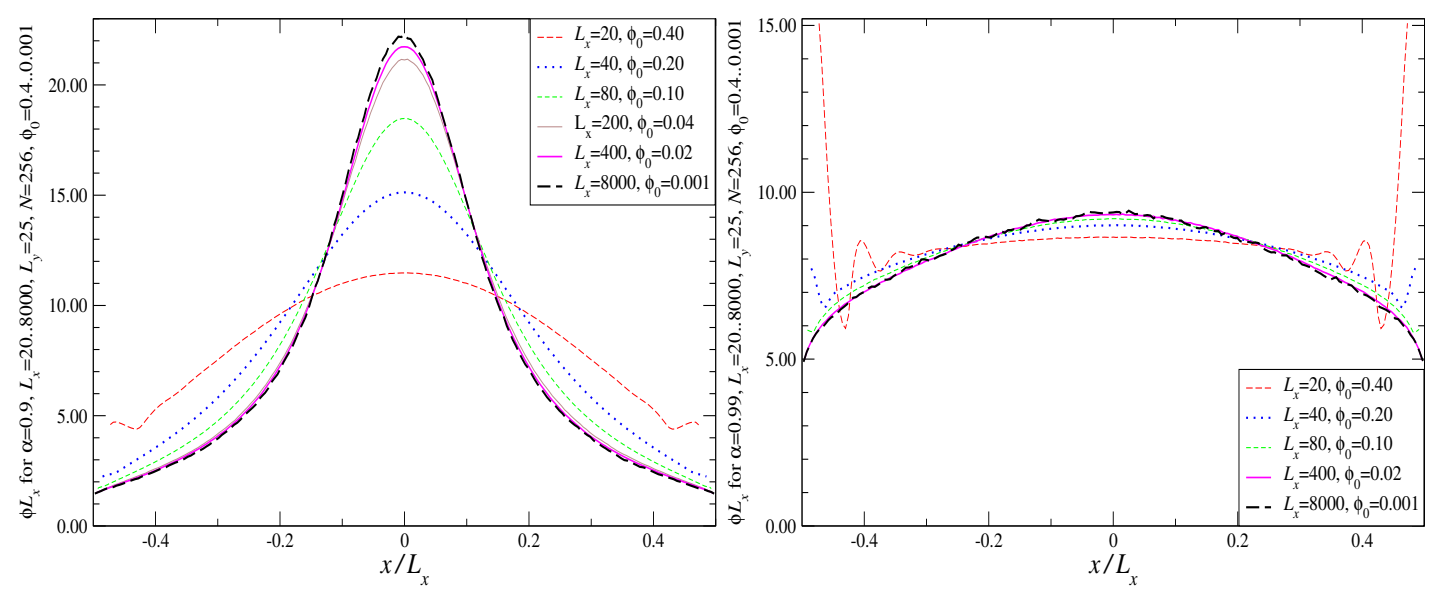

Figure 6.14.: Spatial profile of the rescaled area fraction (density) $\phi(x) L_{x}$ for fixed inelasticities $(\alpha=$ 0.9 in the left graph, $\alpha=0.99$ in the right graph) for various system sizes $L_{x}$. For large $L_{x}\left(\phi_{0} \lesssim 0.01\right)$ the density profiles fall on top of each other. We observe a rising inhomogeneity as the systems become more dilute. For very dilute systems $\left(\phi_{0} \lesssim 0.01\right)$ we observe data collapse.
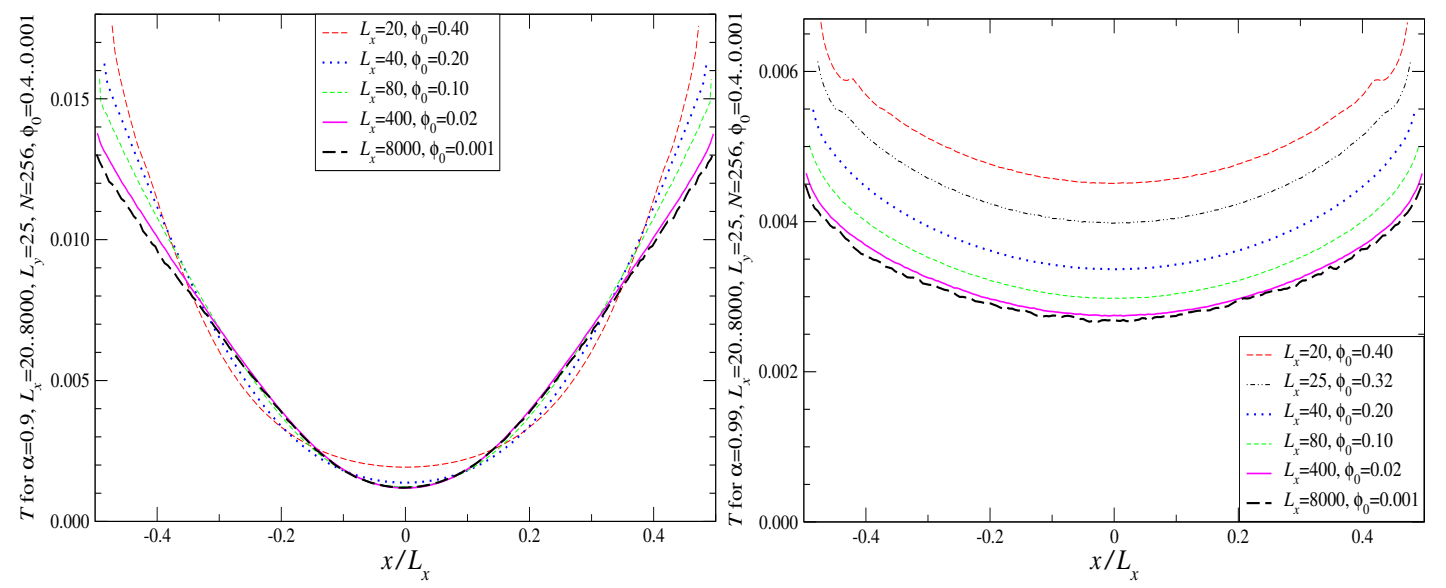

Figure 6.15.: Spatial profile of the temperature profiles $T(x)$ for the same systems as in Figs. 6.14. Here, the shape of the profile changes considerably less than for the density profiles. Again, we observe scaling for $\phi_{0} \lesssim 0.01$.

of the profiles for the more inelastic $(\alpha=0.9)$ case only. For $\alpha=0.99$ the shape is almost unchanged for all sizes of the box edge $L_{x}$. Here, too, we observe scaling in the dilute limit.

\section{Heat Flux}

In Figs. 6.18 we compare the heat flux for two fixed inelasticities (left: $\alpha=0.9$, right: $\alpha=$ 0.99 ) and various sizes of the box edge $20 \leq L_{x} \leq 8000$ (or area fractions $0.001 \leq \phi_{0} \leq 0.4$ ). 

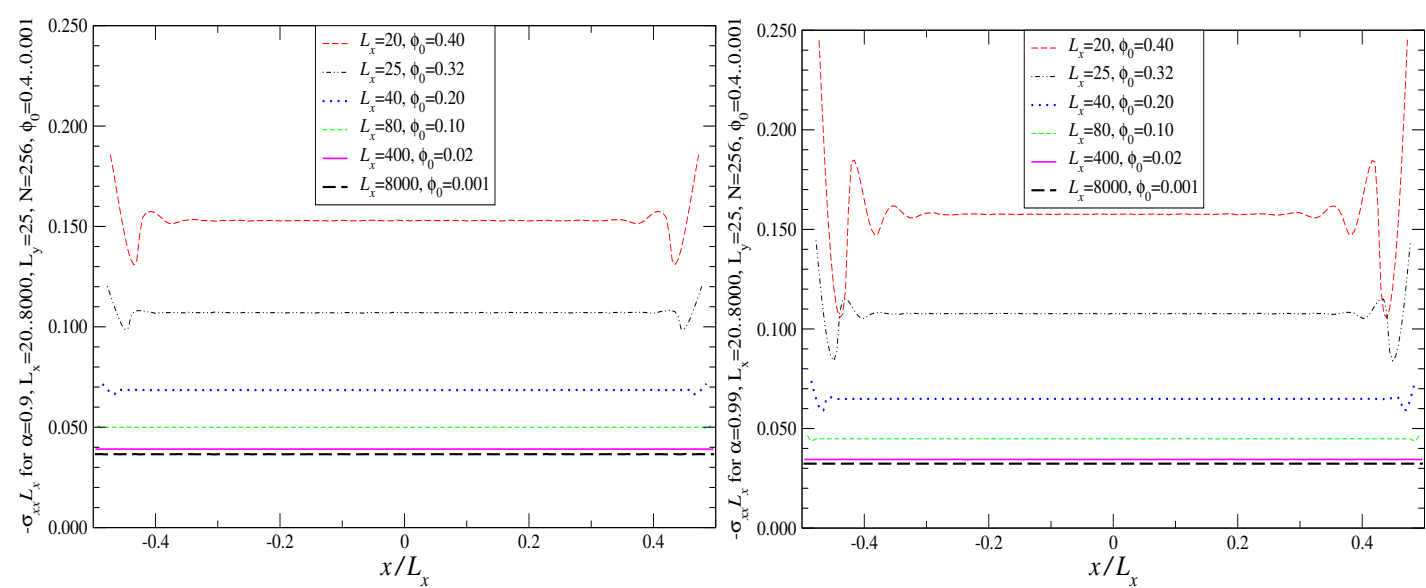

Figure 6.16.: Spatial profile of the $x x$-component of the rescaled stress tensor $-\sigma_{x x}(x) L_{x}$ for the same systems as in Figs. 6.14. The shape of the profiles does not change significantly but the mean value of the stress decreases with decreasing densities. For $\phi_{0} \lesssim 0.01$ we observe scaling.
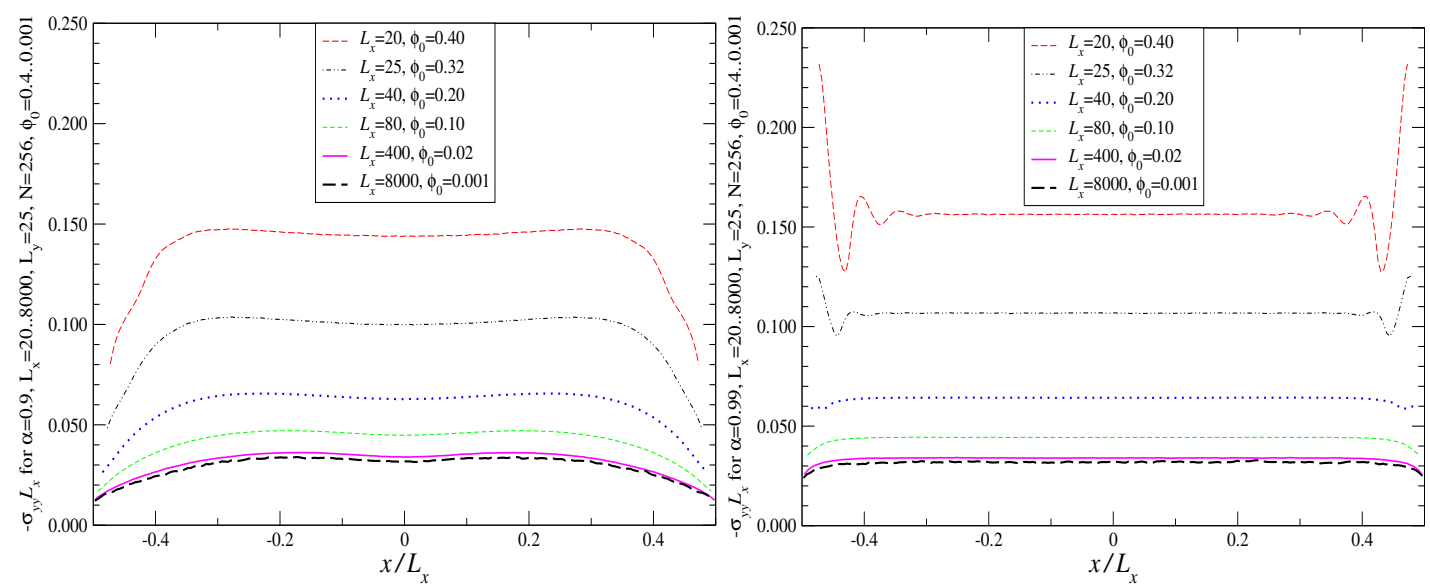

Figure 6.17.: Spatial profile of the $y y$-component of the rescaled stress tensor $-\sigma_{y y}(x) L_{x}$ for the same systems as in Figs. 6.14. The shape of the profiles does not change very much for $\alpha=0.99$ but it does for $\alpha=0.9$. For $\phi_{0} \lesssim 0.01$ we observe scaling.

Since the temperature profiles are quadratic in $x / L_{x}$ in first approximation we expect - inspired by Fourier's law of thermal conductance - that the heat flux profile should be approximately linear and proportional to $L_{x}^{-1}$ when plotted as a function of $x / L_{x}$. As will be discussed in chapter 8 Fourier's law has to be generalized for granular materials but it still gives the correction intuition. As predicted we find that the heat flux scales with $L_{x}^{-1}$ for low enough densities. For higher densities the scaling suggested captures the correct order of magnitude. In good approximation the profiles are linear in all cases but the slope becomes greater for denser systems. In the dilute limit we observe a slope that is independent of the density. 

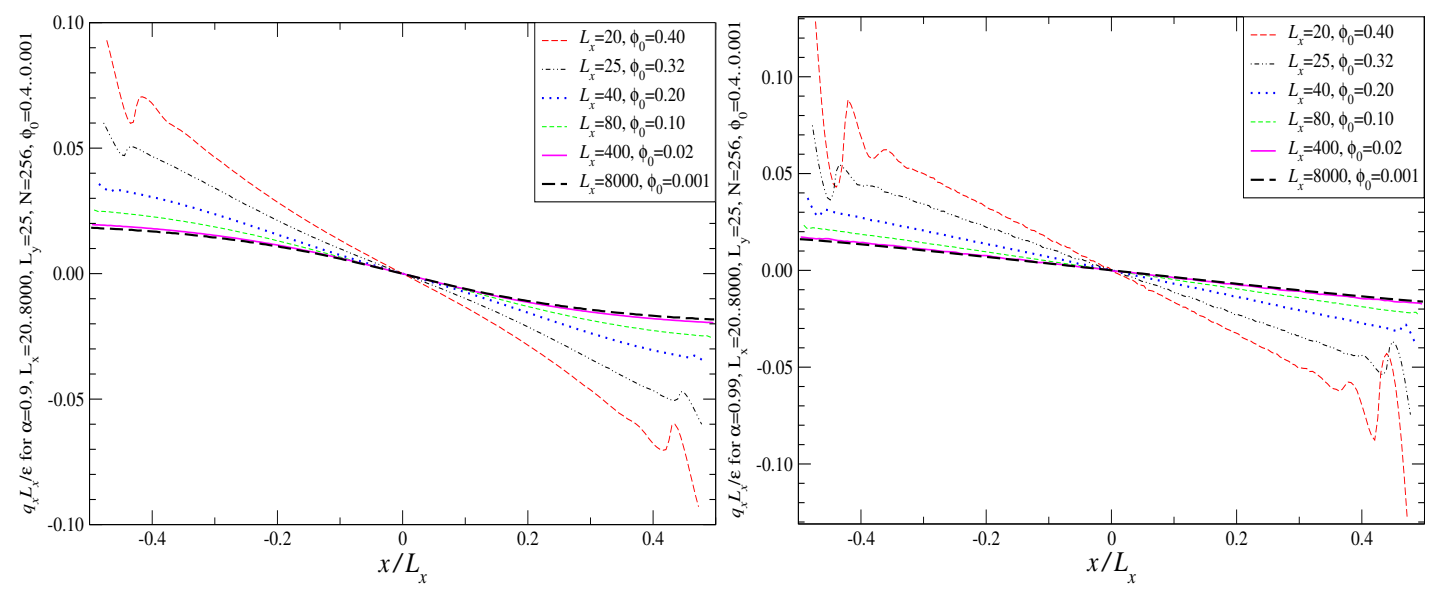

Figure 6.18.: Spatial profile of the rescaled heat flux $q_{x}(x) L_{x} / \varepsilon$ for the same systems as in Figs. 6.14 (for fixed inelasticities [ $\alpha=0.9$ in the left graph, $\alpha=0.99$ in the right graph] for various system sizes $L_{x}$ ). Here, too, the scaling suggested captures the correct order of magnitude. In good approximation the profiles are linear in all cases but the slope becomes greater for denser systems. In the dilute limit we observe a slope that is independent of the density.

\section{Local Energy Loss}

In Figs. 6.19 we show the rescaled energy loss rate $\zeta$ (as in the first row of Figs. 6.11) for two fixed coefficients of restitution (left: $\alpha=0.9$, right: $\alpha=0.99$ ) and various sizes of the box edge $20 \leq L_{x} \leq 800$, corresponding to global area fractions $0.4 \geq \phi_{0} \geq 0.01$. For both inelasticities we see that the maximum dissipation is in the middle of the system for low densities while it is not in the middle for higher densities: The curvature of the energy loss $\zeta$ in the middle of the system changes its sign when the global area fraction is increased from $\phi_{0}=0.1$ to $\phi_{0}=0.2$ (for $\alpha=0.9$ ) or $\phi_{0}=0.3$ (for $\alpha=0.99$ ). While the scaling suggested captures the correct order of magnitude for all systems, we observe excellent scaling for the dilute limit only.

\section{Gradients}

Considering the overall scaling of the gradients with $L_{x}$, remember that we do not consider systems of fixed densities and varying $L_{x}$ but rather systems of fixed line density $\lambda=N / L_{y}$. That means that the (global) density is proportional to $L_{x}^{-1}$, while the mean temperature of the system is independent of $L_{x}$ for low densities according to Eq. (3.13). Therefore we expect the gradient of the density to be proportional to $L_{x}^{-2}$ when plotted as a function of $x / L_{x}$, while the gradient of the temperature should be proportional to $L_{x}^{-1}$ when plotted as a function of $x / L_{x}$. See also the discussion of the low density limit in Sec. 6.9.2. 

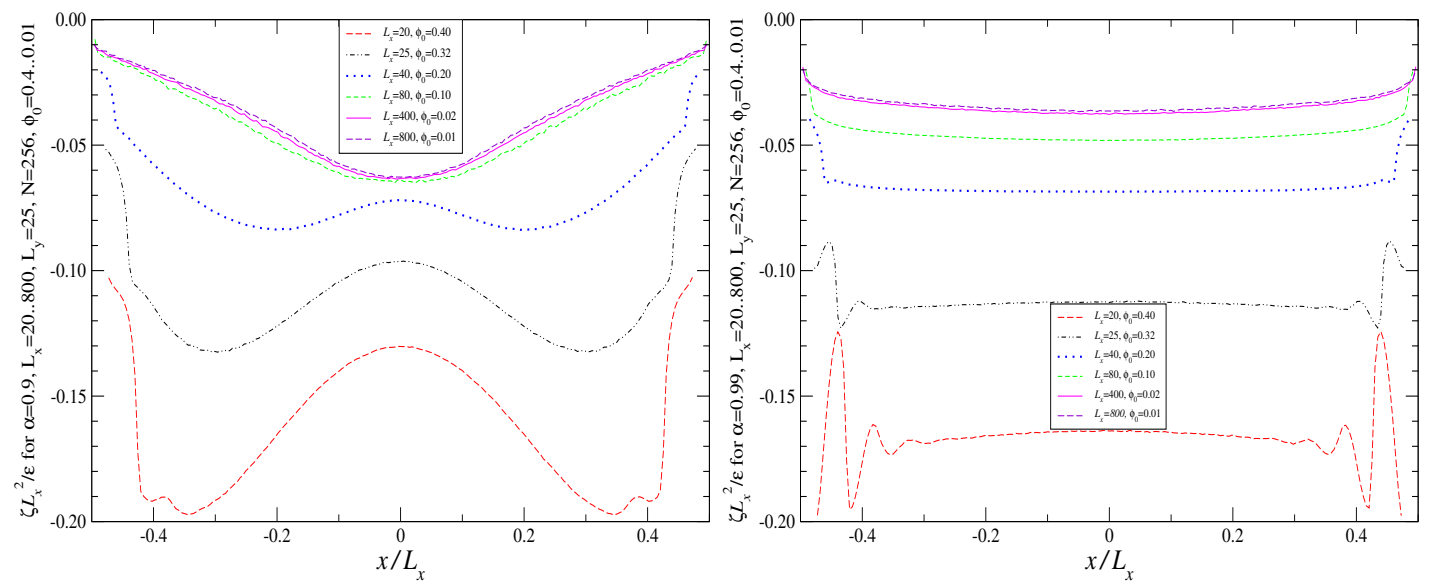

Figure 6.19.: Spatial profile of the rescaled dissipation rate $\zeta L_{x}^{2} / \varepsilon$ for the same systems as in Figs. 6.14 (for fixed inelasticities [ $\alpha=0.9$ in the left graph, $\alpha=0.99$ in the right graph] for various system sizes $L_{x}$ ). For dilute enough systems we observe an approximate data collapse.
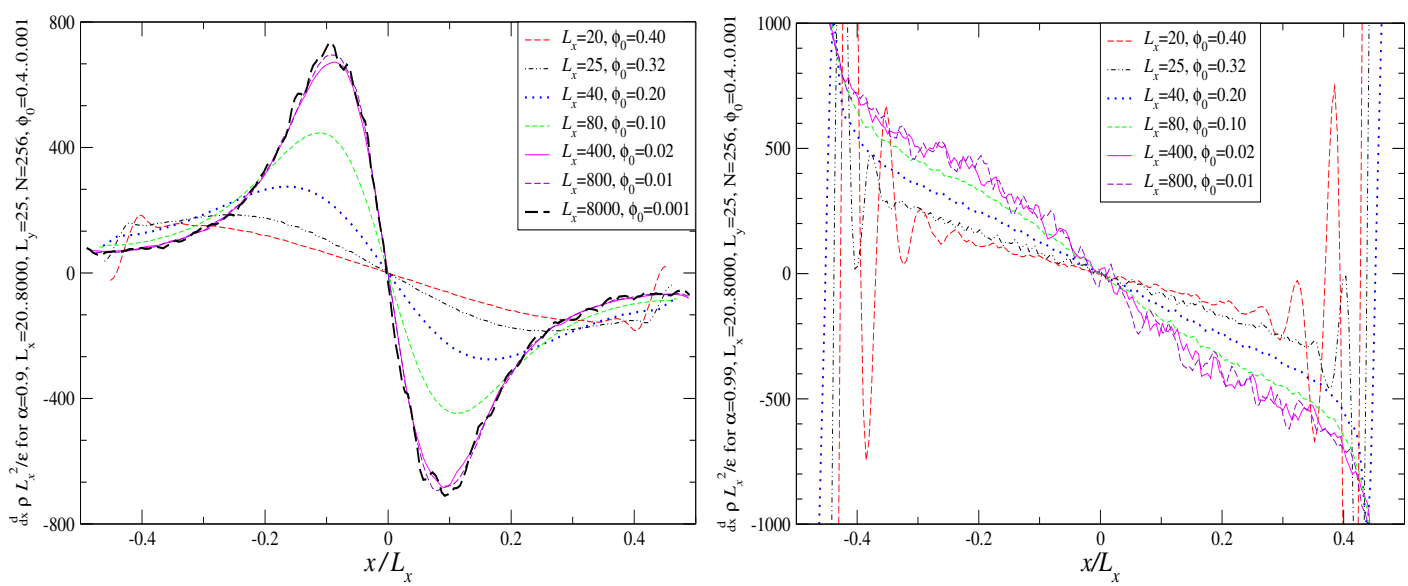

Figure 6.20.: Spatial profile of the rescaled density gradient $\frac{d}{d x} \rho(x) L_{x}^{2} / \varepsilon$ for the same systems as in Figs. 6.14 (left: $\alpha=0.9$, right: $\alpha=0.99$ ). Here, too, the scaling suggested captures the correct order of magnitude for all densities while the shape of the profile strongly depends on the system size/density. In the dilute limit the data collapse becomes excellent.

Finally, Figs. 6.20 and 6.21 show the smoothed rescaled gradients for various sizes of the box edge $L_{x}$ for the same systems presented in Figs. 6.14. Again, we observe scaling for the dilute limit. For the quasi-elastic systems in the right graphs $(\alpha=0.99)$ we find that the density gradient as well as the temperature gradient are linear to first approximation. While the temperature gradients approximately scale for all densities, the density gradients are steeper for more dilute systems. For moderately inelastic systems ( $\alpha=0.9$, left graphs) only the temperature gradients are approximately linear for all densities while the density gradients change from linear profiles for dense systems to an oblique " $z$ " shape for dilute systems. 

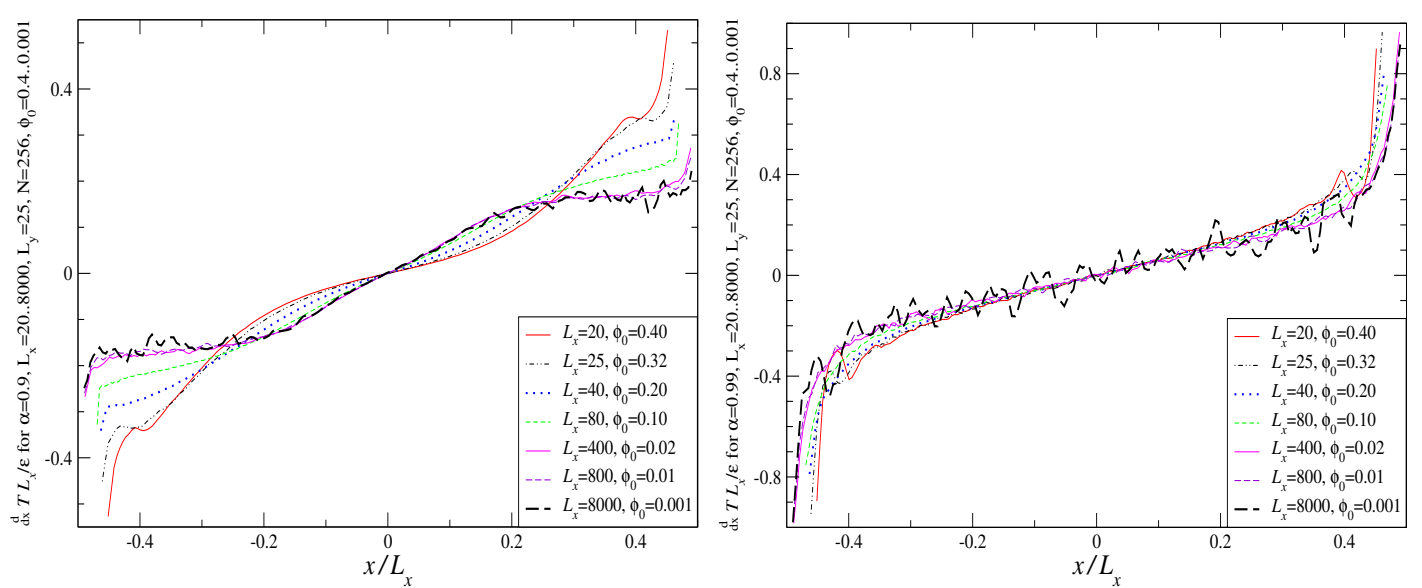

Figure 6.21.: Spatial profile of the rescaled temperature gradient $\frac{d}{d x} T(x) L_{x} / \varepsilon$ for the same systems as in Figs. 6.14 (left: $\alpha=0.9$, right: $\alpha=0.99$ ). The data collapse captures the correct order of magnitude for all densities and also the shape of the profile does not depend too much on the system size/density. In the dilute limit the data collapse becomes excellent again.

\subsection{Summary of Scaling Behavior}

We have presented hydrodynamic fields of the density, temperature, stress tensor, heat flux, energy loss, and gradients of density and temperature for a wide range of parameters in vibrated systems. These profiles are by no means universal but depend on the specific choice of the parameters. Nevertheless for the quasi-elastic limit and/or the dilute limit we were able to extract scaling laws that will be summarized in the following.

\subsubsection{Quasi-Elastic Limit}

Driving the system with a driving velocity $v_{\mathrm{dr}}=\varepsilon$ (where $\varepsilon=1-\alpha^{2}$ ) as suggested by the energy balance equation (3.13) works well for systematically studying quasi-elastic systems as this driving leads to a final temperature that is almost independent of the coefficient of restitution.

\section{Density}

The global density is, of course, independent of the coefficient of restitution. However, the shape of the profile does depend on the coefficient of restition. To first order in $\varepsilon$, the (middle of the) profile is given by

$$
\phi(x)=\phi(0)-c_{\phi} \varepsilon\left(x / L_{x}\right)^{2},
$$


where $\phi(0)$ is the area fraction in the middle of the system. $c_{\phi}$ is a positive constant that does not depend on $\varepsilon$ but depends slightly on $L_{x}$. Of course $\phi(0)$ is a function of $\varepsilon$. This is confirmed by simulations: The insets of Figs. 6.6 show two parameter fits according to Eq. 6.4 to the measured density profiles. Clearly, the agreement is very good in the middle of the systems.

\section{Temperature}

The global temperature is approximately independent of the coefficient of restitution if the system is driven with a driving velocity $v_{\mathrm{dr}}=\varepsilon$. Simulations show that the temperature is not exactly independent of $\alpha$ but instead better approximated by $(1+\alpha)^{2}$.

Similarly to the density profile, to first order in $\varepsilon$, the (middle of the) temperature profile is given by

$$
T(x)=T(0)+c_{T} \varepsilon\left(x / L_{x}\right)^{2},
$$

where again $T(0)$ is the temperature in the middle of the system (i.e. the minimum of the temperature) and $c_{T}$ is a positive constant that does not depend on $\varepsilon$ but depends slightly on $L_{x}$. Again, the minimum of $T(0)$ depends on $\varepsilon$. A comparison with simulations, see the insets of Figs. 6.7 where two parameter fits according to Eq. (6.5) are shown, yields very good agreement in the middle of the systems.

\section{Stress Tensor}

The stress is found to be diagonal, $\sigma_{x y}(x) \approx 0$. In the quasi-elastic limit the anisotropy $\sigma_{x x}(x) \neq \sigma_{y y}(x)$ vanishes and $\sigma_{x x}(x) \approx \sigma_{y y}(x)$ (except for boundary effects). The $x x$ component is almost constant throughout the systems for all coefficients of restitution. The $y y$-component is close to constant in the quasi-elastic limit only. For higher inelasticities $-\sigma_{y y}$ is significantly smaller closer to walls, goes through a maximum and displays a dip in the middle of the systems. Its overall magnitude is smaller than that of $-\sigma_{x x}$. The kinetic parts of the $x x$ - and $y y$-components of the stress tensor are equal to $-\rho(x) T_{x}(x)$ and $-\rho(x) T_{y}(x)$, respectively. Therefore, we expect the stress tensor components to also be approximately proportional to $(1+\alpha)^{2}$ which is confirmed by the simulations for the full range of $\alpha$.

\section{Heat Flux, Local Energy Loss, and Gradients}

Simulations confirm that the heat flux in the $y$-direction vanishes, $q_{y}(x) \approx 0$, and that $q_{x}(x)$ is anti-symmetric in $x$ as expected. In the elastic limit we find $q_{x}(x)$ to be close to linear in $x$ and proportional to $\varepsilon$. As expected, also the local energy loss $\zeta(x)$ shows a linear dependence on $\varepsilon$, 
too, to first order in $\varepsilon$. The $y$-component of the density gradient, $\frac{d}{d y} \rho(x)$, vanishes as expected for all systems. The $x$-component is proportional to $\varepsilon$ (to first order in $\varepsilon$ ) as suggested by Eq. (6.4). The behavior of the temperature gradient is the same as for the density gradient, cf. Eq. (6.5), to lowest order in $\varepsilon$.

\subsubsection{Low Density Limit}

We have seen that, once $L_{x}$ is large enough, all hydrodynamic field profiles can be collapsed onto master curves for each hydrodynamic field when plotted versus $x / L_{x}$. More precisely, we find an integer number $n$ for each hydrodynamic field $\psi$ such that $L_{x}^{n} \psi\left(x / L_{x}\right)$ becomes independent of the size of the box edge $L_{x}$. This scaling has been observed for low global area fractions, $\phi_{0} \lesssim 0.01$, as discussed above. In the following we will summarize the results and state $n$ for all hydrodynamic fields.

\section{Density}

We have seen that the relative local area fraction $L_{x} \phi(x)$ plotted versus $x / L_{x}$ exhibits scaling. Thus, $n=1$. Before we go on to the other hydrodynamic fields, we study the scaling of the density profile in more detail in Fig. 6.22, where we compare density profiles $\phi(x) / \phi_{0}$ of systems with the same degree of inelasticity $\alpha=0.9$ and the same line density $\lambda=10.24$, but different values of the box edge $1280 \leq L_{x} \leq 20000$, corresponding to global area fraction $\phi_{0}$ between $6 \cdot 10^{-3}$ and $4 \cdot 10^{-4}$. The relative local area fraction $\phi / \phi_{0}$ is seen to be a function of $x / L_{x}$ only and does not depend separately on $L_{x}$ and $\phi_{0}$. For global area fraction $\phi_{0} \gtrsim 0.01$ then the data collapse ceases to hold and the hight of the peak reduces as can be seen in Figs. 6.14 in Sec. 6.2.

A hydrodynamic approach to quasi elastic, driven granular gases by Grossman et al. in Sec. II D in Ref. [GZBN97] predicts for low-density systems that the mean free path in units of $L_{x}$ depends only on the line density according to $\ell / L_{x}=1 /(\sqrt{8} \lambda)$. When computed for the situation of Fig. 6.22, one gets the small numerical value $\ell / L_{x} \approx 0.03$, indicating that $\ell$ is not a relevant length scale for the master curve of the rescaled spatial density profiles. The theory of [GZBN97] also makes a prediction for the relative local area fraction $\phi(x) / \phi_{0}$ in terms of the solution of a first-order differential equation. This differential equation includes one free parameter, which we have fitted in order to match the solution (dotted line in Fig. 6.22) at $x=0$ with the master curve from our simulations. The agreement is reasonable, showing that inelasticities of $\alpha=0.9$ are at the borderline of the scope of this otherwise powerful approach to quasi elastic, driven granular gases. 


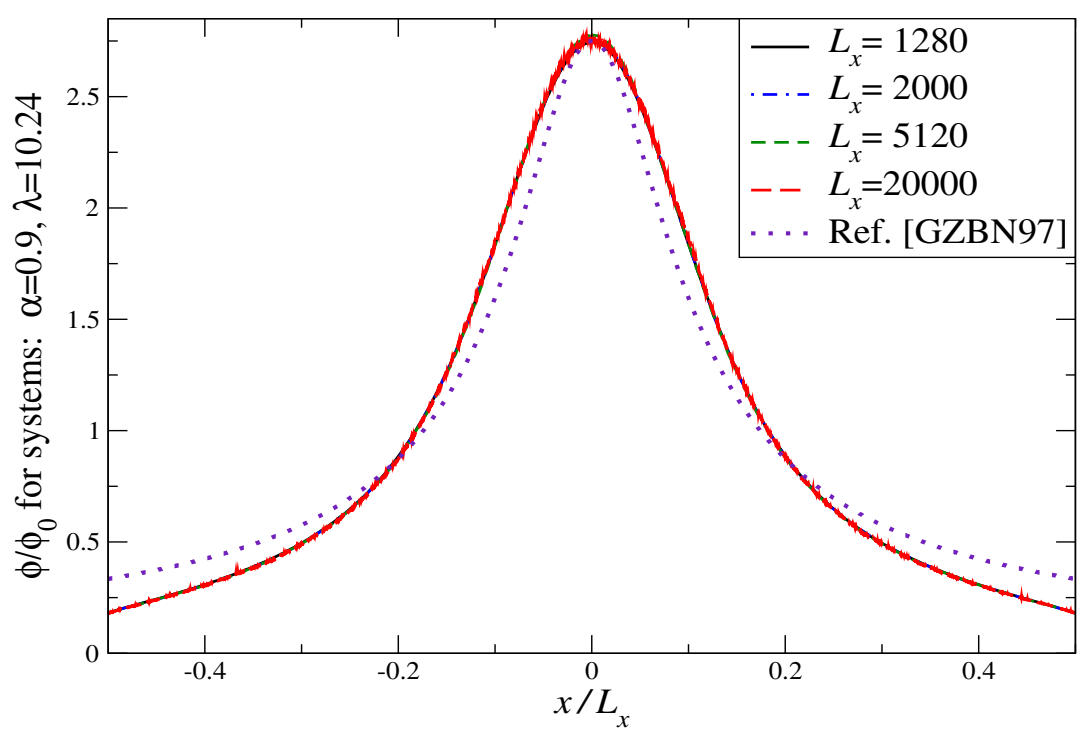

Figure 6.22.: Master curve for rescaled local area fraction in different low-density systems with the same line density $\left[\alpha=0.9, \lambda=10.24, \ell / L_{x} \approx 0.03\right]$. The dotted line corresponds to the theoretical prediction of Ref. [GZBN97].

\section{Temperature, Stress Tensor, Heat Flux, Energy Loss, and Gradients}

As expected, cf. Eq. (3.13), the temperature profiles do not depend (very much) on $L_{x}$ and exhibit scaling without any rescaling, so $n=0$. The scaling is very good for dilute systems. For the stress tensor $n=1$, i.e. $\sigma\left(x / L_{x}\right) \propto L_{x}^{-1}$, since in the dilute limit we expect the ideal gas law to hold. Again, scaling is very good for dilute systems while it yields merely the correct order of magnitude for higher densities. For the heat flux $n=1$, i.e. $q_{x}(x) \propto L_{x}^{-1}$. This can be understood when we take a look at the rate at which energy is injected into the system at the walls. From Eq. (3.4) in Sec. 3.1 we know that the average energy a particle gains when colliding with a wall is proportional to $L_{x}^{-1}$. On the other hand, the density at the wall is proportional to $L_{x}^{-1}$ as has been discussed above. Therefore we expect the heat flux to be proportional to $L_{x}^{-2}$, i.e. it is proportional to $L_{x}^{-1}$ when plotted versus $x / L_{x}$. In the middle of the system the heat flux vanishes. For the energy $\operatorname{loss} \zeta(x)$ we find $n=2$. As discussed above, $\zeta(x)$ integrated over the entire system must be twice the heat flux at either wall. Therefore, $\zeta\left(x / L_{x}\right) \propto L_{x}^{-2}$. Differentiating Eq. (6.4) in Sec. 6.9.1 with respect to $x$ we see that, as a function of $x$ (rather than $x / L_{x}$ ), the density gradient would have the same $n$ as the density itself. Plotted as a function of $x / L_{x}$, as we have done, $n$ increases by one, because $x^{2}$ reduces to $x$ while the power of $L_{x}$ does not change. Thus, we find $n=2$ or $\frac{d}{d x} \rho\left(x / L_{x}\right) \propto L_{x}^{-3}$ With the same arguments as for the density gradient the value $n$ for the temperature gradient is increased by one compared to the temperature itself, i.e. $n=1$ or $\frac{d}{d x} T\left(x / L_{x}\right) \propto L_{x}^{-2}$. 


\section{Local Equation of State in Vibrated Systems}

Hydrodynamic approaches [GZBN97, BRMM00, LMS02b] to granular gases as introduced in chapter 4 require the knowledge of constitutive relations in order to close the fundamental set of equations (4.1). In two dimensions these four equations contain eleven hydrodynamic fields: the density $\rho$, the $2 \mathrm{D}$ velocity field $\boldsymbol{V}$, the temperature $T$, the $2 \times 2$ stress matrix $\boldsymbol{\sigma}$, the vector of the heat flux $\boldsymbol{q}$, and the energy loss $\zeta$. Very often the stress tensor is assumed to be symmetric which leaves us with ten hydrodynamic fields. The standard way to reduce the number of fields starts by decomposing the stress tensor into a hydrostatic part and one that depends on the velocity field. Since a homogeneous velocity field is equivalent to a fluid at rest the stress tensor can only depend on spatial derivatives of the velocity field. Furthermore, the hydrostatic part is usually assumed to be isotropic and referred to as the hydrostatic pressure $p$ - an assumption that is only a rough approximation for granular systems as we have seen in chapter 6 . For the sake of simplicity we consider the pressure to be isotropic here. Using a Stokes friction ansatz this allows us to write the stress tensor of a viscous fluid in two dimensions as

$$
\sigma_{k l}=-p \delta_{k l}+\eta_{S}\left[\partial_{k} V_{l}+\partial_{l} V_{k}-\left(\nabla_{\boldsymbol{r}} \cdot \boldsymbol{V}\right) \delta_{k l}\right]+\eta_{B}\left(\nabla_{\boldsymbol{r}} \cdot \boldsymbol{V}\right) \delta_{k l}
$$

where $\eta_{S}$ is the shear viscosity and $\eta_{B}$ is the bulk viscosity. Eq. 7.1 reduces the number of hydrodynamic fields to eight. [Note: Inserting Eq. (7.1) into the momentum balance equation (4.6) and assuming $\eta_{S}$ and $\eta_{B}$ to be constant in space yields the Navier-Stokes equation in 2 dimensions. Also note that the velocity field $\boldsymbol{V}$ vanishes in all systems studied in this thesis, reducing the Navier-Stokes approximation of the stress tensor, Eq. (7.1), to the hydrodynamic pressure.] What remains to be done in order to close the hydrodynamic equations is to relate the pressure $p$ as well as the heat flux $\boldsymbol{q}$ and the energy loss $\zeta$ to the remaining four fields for the density $\rho$, the velocity $\boldsymbol{V}$, and the temperature $T$. In the last chapter we have already estimated the energy loss $\zeta$ in terms of the density and the temperature, cf. Eq. (6.3). In this chapter we will focus on relating the pressure to these fields. In the next chapter we will do so for the heat flux. 


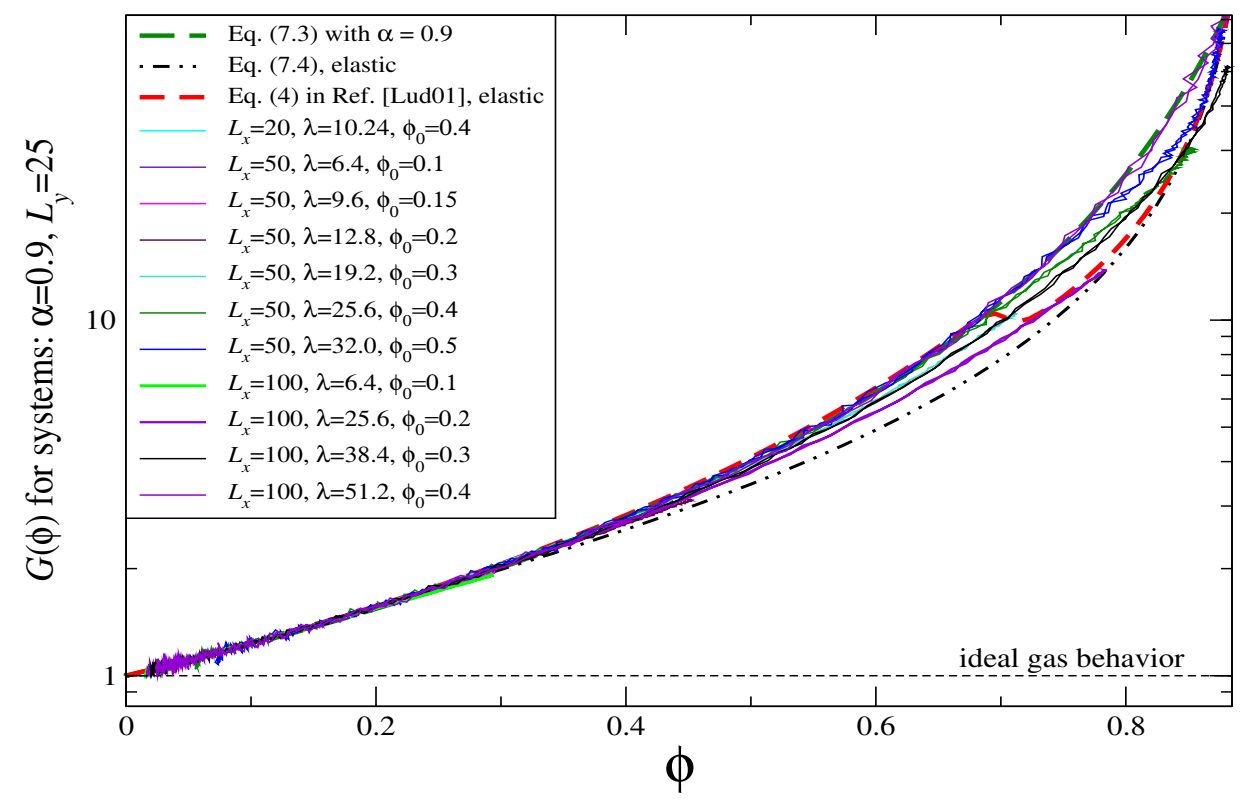

Figure 7.1.: Semilogarithmic parametric plots of the function $G(\phi)$ from the local equation of state (7.2) for different systems $\left[\alpha=0.9, L_{y}=25,0.01 \leq \ell / L_{x} \leq 0.05\right]$. Also shown are the theoretical predictions from Eqs. (7.3), (7.4), and Eq. (4) in Ref. [Lud01].

\subsection{Deriving a Constitutive Equation}

In the last chapter it has been shown that a driven granular gas is intrinsically inhomogeneous. Therefore it is only natural to investigate how the local values of the granular temperature $T(x)$, pressure $p(x)$, and density $\rho(x)$ —resp. area fraction $\phi(x)=\rho(x) \pi / 4$ in our units-are related to each other. For this purpose we observe in Figs. 6.2 and 6.3 that $T(x), p(x)$, and $\phi(x)$ are all symmetric in $x$. Moreover, and this is crucial, $\phi(x)$ is monotone in $x$ for either sign of $x$ (except for a boundary layer of approximately one diameter in width close to a driving wall, which we ignore). Therefore one can invert the function $\phi(x)$ for positive $x$. Upon inserting $x=\phi^{-1}(\phi(x))$ into the local pressure and temperature, we arrive at the constitutive equation

$$
\frac{p(x)}{\rho(x) T(x)}=G(\phi(x))
$$

with some function $G$. 


\subsection{Universality of the Constitutive Equation for Low to Moderate Inelasticities}

Fig. 7.1 shows parametric plots on a semilogarithmic scale of the function $G$ with the values of $p(x) /[\rho(x) T(x)]$ plotted against those of $\phi(x)$ for all $x$ (except for those in the boundary layer mentioned above). In order to utilize a broad range of $\phi$-values, Fig. 7.1 contains data from 11 different systems, all of which have the same coefficient of restitution $\alpha=0.9$ and the same height $L_{y}=25$, but different widths $L_{x}$ and different global area fractions $\phi_{0}$. For not too large values of $\phi$ these data merge quite nicely, indicating that there is only a weak dependence of $G$ on the global system parameters $L_{x}$ and $\phi_{0}$ in the corresponding parameter range. In this case the constitutive equation (7.2) can be interpreted as the local equation of state of the system. The horizontal line in Fig. 7.1 marks ideal-gas behavior, from which $G$ deviates due to the collisional contribution to the pressure. These deviations increase significantly with increasing $\phi$.

The long-dashed dark green line in Fig. 7.1 corresponds to the function

$$
G(\phi)=1+(1+\alpha) \phi \chi,
$$

where $\chi$ stands for the pair correlation function at contact of the associated elastic hard-sphere gas in thermal equilibrium. Since $\chi$ is not known exactly, we estimate it by the Henderson [Hen75] approximation, Eq. (3.6), for numerical purposes. In the context of granular gases, Eq. (7.3) already occurred in Ref. [JR85] and later in a global equation of state for the homogeneous situation of a freely cooling granular gas [GS95]. In Ref. [SM01] the pair correlation function has been studied in a homogeneously driven inelastic system with periodic boundary conditions. It was found to be nearly independent of the coefficient of restitution and well approximated by the Henderson approximation, Eq. (3.6). In Ref. [BT02b] this form of $G$ was used in the local equation of state (7.2) to get a theoretical prediction for $p(x)$ from simulations of $T(x)$ and $\phi(x)$ in a driven granular gas. Fig. 7.1 reveals that this works generally quite well for up to rather high local area fractions $\phi(x) \lesssim 0.5$. In even denser systems agreement still holds for the well fluidized parts. Deviations from Eq. (7.3) start to occur when entering the transition zones to the frozen-out stripe of particles in the center of these high-density systems. The dashed-double-dotted black line in Fig. 7.1 corresponds to the interpolation formula [GZBN97]

$$
G(\phi)=\frac{\phi_{c}+\phi}{\phi_{c}-\phi}
$$

for $0 \leq \phi<\phi_{c}$, which connects the behavior of dilute (van der Waals) and dense (ordered) elastic hard-sphere systems. Here $\phi_{c}:=\pi /(2 \sqrt{3}) \approx 0.91$ denotes the area fraction for ordered closed packings in two dimensions. Eq. (7.4) was applied to quasi-elastic granular gases in Ref. [GZBN97], and even for our simulations with $\alpha=0.9$ there is agreement with the local data

\footnotetext{
${ }^{1}$ The expression (7.3) for $G$ with $\alpha=1$ is known from the exact equation of state $p /(\rho T)=1+2^{d-1} \phi \chi$ of a $d$-dimensional elastic hard-sphere gas in thermal equilibrium [HM86].
} 


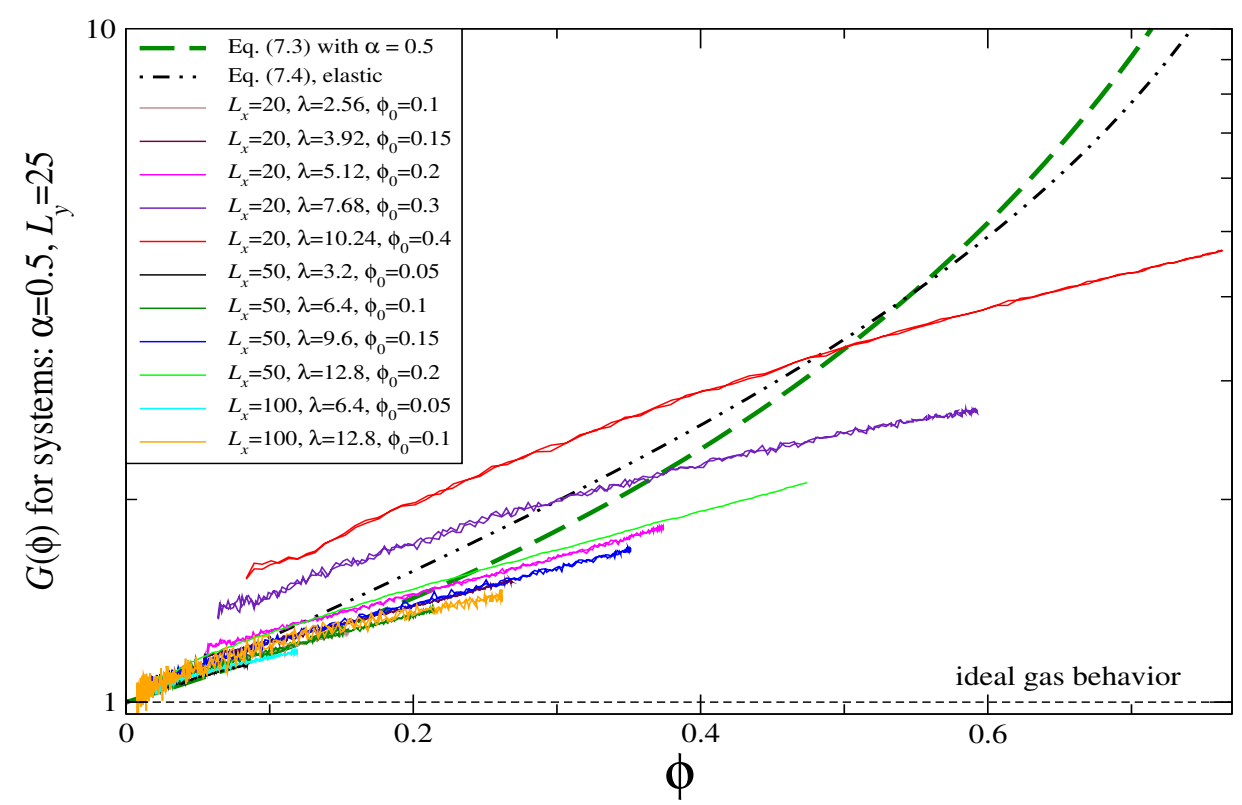

Figure 7.2.: Same as Fig. 7.1 but for more inelastic systems $\left[\alpha=0.5, L_{y}=25,0.01 \leq \ell / L_{x} \leq 0.1\right]$.

in the low-density regions up to $\phi(x) \lesssim 0.4$. In addition, the most dense regions of regularly ordered, frozen-out particles in the center of the high-density systems are described correctly, too. Yet another interpolation formula for $G$, which is rather accurate for an elastic hard-sphere gas even in the vicinity of the freezing transition, was put forward in Eq. (4) in [Lud01] and is depicted by the dashed red line in Fig. 7.1. Thus, the crossover between fluidized and frozenout behavior in the inelastic, driven systems, originating from the transition zone at the border of the solidified stripe of particles in the system center, is very smooth in comparison to the coexistence region of the freezing transition for an equilibrated elastic gas. Moreover, the location of the crossover depends on the global system parameters. Since both interpolation formulae, (7.4) and the one from [Lud01], were tailored for elastic systems in thermal equilibrium, it is surprising to find such a good agreement with the local data for driven inelastic hard-sphere gases with restitution $\alpha=0.9$.

\subsection{Non-Universality of the Constitutive Equation for Higher Inelasticities}

When lowering the coefficient of restitution $\alpha$ from 0.9 to 0.8 (not shown), there are only little changes to the plot in Fig. 7.1. In particular, the spread of the data pertaining to different systems increases, even for low densities. Still one may be inclined to interpret (7.2) as a local equation of state-at least approximately. This situation is intermediate to the previous with 
$\alpha=0.9$ and the following for strongly inelastic systems with coefficient of restitution $\alpha=0.5$. Indeed, Fig. 7.2 reveals major discrepancies in the local pressure, which are due to the systems with global area fractions above $\phi_{0} \approx 0.2$. The discrepancies occur even at positions in the sample where the local area fractions are below 0.2 and where all the more dilute systems, i.e. those with $\phi_{0} \leq 0.2$, agree reasonably with the proposal (7.3). The concept of a local equation of state is therefore not sustainable any more for such strongly inelastic systems, and (7.2) merely plays the role of a local constitutive equation, which depends in addition on the global system parameters. 


\section{Fourier's Law and its Generalization for Vibrated Systems}

It is a characteristic feature of hydrodynamics of inelastically colliding particles that a heat current can be generated not only by a nonuniform temperature but also by density inhomogeneities. If the spatial variations of temperature or density are restricted to long wavelengths, one would expect a gradient expansion to hold. The simplest constitutive equation for the heat flux is thus a straightforward generalization of Fourier's law of thermal conductance, as discussed in the literature, cf. Refs. [CC70, JR85, BMD96, DBS97, SG98]. Chapman-Enskog expansions of both the Boltzmann and the Boltzmann-Enskog equations predict that the heat flux of an inelastic system is given by

$$
\boldsymbol{q}(\boldsymbol{r}, t)=-\kappa \nabla_{\boldsymbol{r}} T(\boldsymbol{r}, t)+\mu \nabla_{\boldsymbol{r}} \rho(\boldsymbol{r}, t),
$$

where $\kappa$ is the heat conductivity and $\mu$ is a new transport coefficient. This new transport coefficient has no analogue for elastic systems, for which Fourier's law of thermal conductance predicts the heat flux to be proportional to the temperature gradient.

This chapter is divided into two parts. First we will compare our simulational data for the heat flux and the local energy loss to a theory by Jenkins and Richman [JR85]. In the second part we will discuss how to extract the transport coefficients from our data, show them as a function of the coefficient of restitution for various densities, and compare the prediction using both transport coefficients to the one by Fourier's law.

\subsection{Comparison of Heat Flux and Energy Loss to the Literature}

Few theoretical descriptions exist for the transport coefficients that include collisional contributions, especially for two-dimensional systems. Brey, Dufty et al. [BMD96, DBS97] have calculated transport coefficients in $d$ dimensions based on kinetic theory but their results are given in terms of integrals to be solved. Ignoring collisional contributions, Sela et al. [SG98] have calculated the transport coefficients by means of kinetic theory up to Burnett order for dilute three dimensional systems. 
To our knowledge the only theoretical calculation yielding the transport coefficients for $2 \mathrm{D}$ systems solely in terms of the system parameters and without assuming extremely dilute systems has been performed by Jenkins and Richman [JR85] back in 1985. They find for small inelasticities $\varepsilon \ll 1$,

$$
\begin{aligned}
& \kappa_{J R}=\left[4 \frac{2+3 \phi \chi r\left(r^{2}-\frac{3}{4} \varepsilon\right)}{\chi(8 r+15 \varepsilon)}(2+3 \phi \chi r)+\frac{8 \phi^{2} \chi r}{\pi}\right] \sqrt{\frac{T}{\pi}} \\
& \mu_{J R}=\frac{3 \pi^{2} \alpha \varepsilon}{4 \chi(8 r+15 \varepsilon)}(2+3 \phi \chi r)\left(2 \chi+\phi \frac{d \chi}{d \phi}\right)\left(\frac{T}{\pi}\right)^{\frac{3}{2}},
\end{aligned}
$$

where $r:=(1+\alpha) / 2$ and, again, $\varepsilon=1-\alpha^{2}$. Similarly, Jenkins and Richman [JR85] calculate the energy loss rate $\zeta$. They obtain

$$
\zeta_{J R}=-16 \varepsilon \phi^{2} \chi(T / \pi)^{3 / 2}
$$

which is the same result as already estimated in Sec. 6.7, Eq. (6.3). For the pair correlation at contact $\chi$ we use the Henderson approximation [Hen75], Eq. (3.6), again. This allows the derivative of $\chi$ by $\phi$ to be calculated analytically. Clearly, from Eqs. (8.2) we see that, to first order in $\varepsilon$, Jenkins and Richman [JR85] predict $\kappa_{J R}$ to be constant in $\varepsilon$, while $\mu_{J R}$ is proportional to $\varepsilon$.

Inserting the local temperature $T(x)$ and local area fraction $\phi(x)$ from our simulations into Eqs. (8.2) and (8.3), we obtain spatially dependent transport coefficients $\kappa_{J R}(x)$ and $\mu_{J R}(x)$ as well as a prediction for the local energy loss $\zeta_{J R}(x)$. In Figs. 8.1 the resulting heat flux (dasheddouble-dotted dark green lines in the main graph) and energy loss (double-dashed-dotted dark green lines in the inset) from Eqs. (8.1) and (8.3) are shown for six different systems. The agreement to the directly measured data (full indigo/magenta lines) is very good for simulations with $\alpha \geq 0.99$. The agreement is reasonable even up to $\alpha \sim 0.96$. For larger inelasticities the theoretical curves capture the correct order of magnitude, but overestimate the curvature of $q_{x}(x)$ and underestimate the maximum of the absolute value of the energy loss $|\zeta(x)|$.

Alternatively we can use the global or mean temperature $T_{0}$ and area fraction $\phi_{0}$ to evaluate Eqs. (8.2). The difference between the resulting constant transport coefficients $\kappa_{J R_{0}}$ and $\mu_{J R_{0}}$ and the local ones $\kappa_{J R}(x)$ and $\mu_{J R}(x)$ is only a few percent for $\alpha \geq 0.99$. The difference in the corresponding heat fluxes is even less, because the strongest inhomogeneities in the transport coefficients occur in the middle of the sample where the gradients of temperature and density vanish. The heat flux, computed using the constant transport coefficients $\kappa_{J R_{0}}$ and $\mu_{J R_{0}}$, is also shown in Figs. 8.1 for comparison (dashed orange lines). The fluctuations of the transport coefficients with $x$ increase with increasing inelasticity, e.g. for $\alpha=0.9$ we find $\kappa_{J R}(0) / \kappa_{J R_{0}} \approx 1.5$. In addition, Figs. 8.1 shows a two-parameter fit (dotted black lines) of Eq. (8.1) to the directly measured data (full indigo lines) that will be discussed in the next section. 

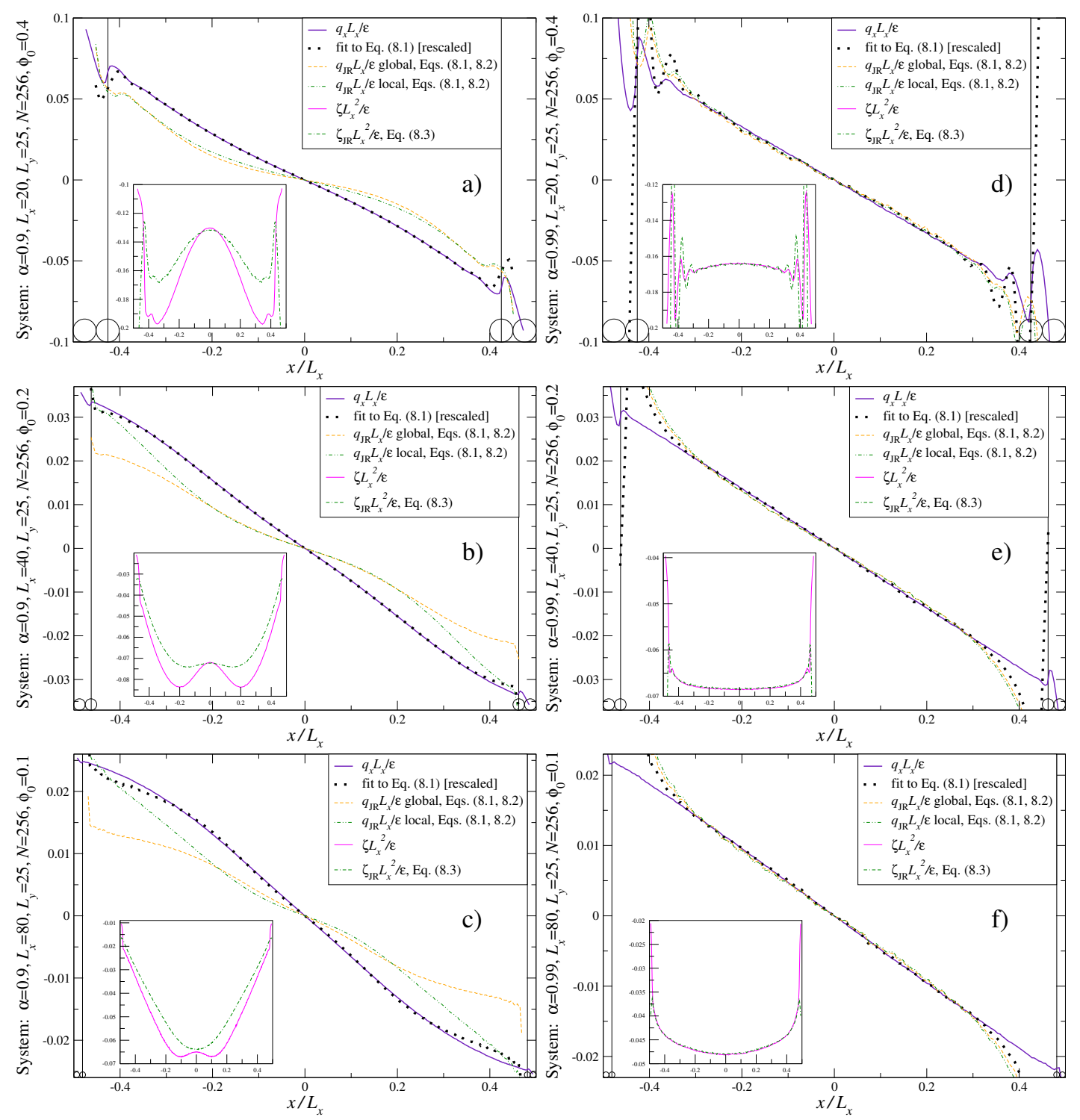

Figure 8.1.: Comparison of the heat flux $q_{x}(x)$ and the local energy loss $\zeta(x)$ from our simulations to the predictions of Ref. [JR85] using local and global transport coefficients. In addition, we show a fit of Eq. (8.1) to our data. Clearly, the agreement between the fit and the measured heat flux is very good for all systems. The theory of Ref. [JR85] also describes the data very well for $\alpha=0.99$ (right graphs) and greater (not shown). For these small inelasticities there is virtually no difference using constant or $x$-dependent transport coefficients in Eq. (8.1). For higher inelasticities (left graphs, $\alpha=0.9$ ) there are significant deviations between the simulations and the predictions of Ref. [JR85]. Additionally, for higher inelasticities inserting the global values $\phi_{0}$ and $T_{0}$ into Eq. (8.2) is significantly worse than inserting the local ones. 


\subsection{Transport Coefficients From Simulations}

\subsubsection{The Fitting Procedure}

To estimate the transport coefficients from our data, we assume that both $\kappa$ and $\mu$ do not depend on the position $x$, i.e. we assume them to be constant throughout the box. It is then straightforward to extract them from a fit of our data to the above expression (8.1) by the following procedure.

From our simulations we know the values of $q_{x}, \partial_{x} T$, and $\partial_{x} \rho$ at a certain number $\tilde{N}$ of positions in the sample, where $\widetilde{N}$ depends on the resolution chosen and $\partial_{x}=d / d_{x}$. We label those $\widetilde{N}$ positions $x_{1}, x_{2}, \ldots, x_{\widetilde{N}}$ and define four $\widetilde{N}$-dimensional vectors

$$
\begin{aligned}
\boldsymbol{Q} & :=\left(q_{x}\left(x_{1}\right), q_{x}\left(x_{2}\right), \ldots, q_{x}\left(x_{\tilde{N}}\right)\right) \\
\boldsymbol{\partial} \boldsymbol{T} & :=\left(\partial_{x} T\left(x_{1}\right), \partial_{x} T\left(x_{2}\right), \ldots, \partial_{x} T\left(x_{\tilde{N}}\right)\right) \\
\boldsymbol{\partial} \boldsymbol{\rho} & :=\left(\partial_{x} \rho\left(x_{1}\right), \partial_{x} \rho\left(x_{2}\right), \ldots, \partial_{x} \rho\left(x_{\widetilde{N}}\right)\right) \\
\boldsymbol{\Delta} & :=\boldsymbol{\partial} \boldsymbol{\rho}-c \boldsymbol{\partial} \boldsymbol{T}, \text { where } c:=\boldsymbol{\partial} \boldsymbol{T} \cdot \boldsymbol{\partial} \boldsymbol{\rho} /|\boldsymbol{\partial T}|^{2} .
\end{aligned}
$$

We want to find those transport coefficients $\kappa$ and $\mu$ that minimize the error $|\chi|^{2}$ when writing

$$
Q=-\kappa \partial T+\mu \partial \rho+\chi=(-\kappa+c \mu) \partial T+\mu \Delta+\chi .
$$

It is easy to see that, by construction, $\Delta$ is perpendicular to $\partial T$, i.e. $\Delta \cdot \partial T=0$. Thus, $\chi$ must be perpendicular to both $\partial \boldsymbol{T}$ and $\Delta$ to minimize the error $|\chi|^{2}$ while fulfilling Eq. (8.5). That leads to the following transport coefficients

$$
\begin{aligned}
& \mu=\boldsymbol{Q} \cdot \boldsymbol{\Delta} /|\boldsymbol{\Delta}|^{2} \\
& \kappa=-\boldsymbol{Q} \cdot \boldsymbol{\partial T} /|\boldsymbol{\partial T}|^{2}+c \mu .
\end{aligned}
$$

These transport coefficients are shown in the left graphs of Figs. 8.2 and 8.3 and will be discussed later. A quantitative measure of the error, i.e. the deviations between the fit and the measured heat flux is given by $|\chi|^{2}$, where $\chi$ can be calculated by inserting Eqs. (8.6) into Eq. (8.5).

To quantify the contribution to the heat flux that is due to density inhomogeneities we compare Eq. (8.1) with the fitted transport coefficients (8.6) to a (one-parameter) fit to Fourier's law. Fitting to Fourier's law is equivalent to setting $\mu=0$ in the expression for $\kappa$ in Eqs. (8.6) and yields $\kappa_{F}=-\boldsymbol{Q} \cdot \boldsymbol{\partial T} /|\boldsymbol{\partial T}|^{2}$ and $\chi_{F}=Q+\kappa_{F} \partial \boldsymbol{T}$. The relative error

$$
R_{1}:=\frac{\left|\chi_{F}\right|}{|\chi|}-1
$$

relates the deviations between the measured data and a fit to Fourier's law $\left|\chi_{F}\right|$ on the one hand to the deviations between the measured data and the two-parameter fit $|\chi|$ on the other hand. 
This gives a quantitative measure for the improvement of the fit when the generation of heat flux due to density inhomogeneities is taken into account in addition to the one generated by temperature gradients. Another quantitative measure for the importance of the new transport coefficient is given by the contribution to the fit that is perpendicular to the temperature gradient. In units of the best fit to Fourier's law it quantifies the relative contribution that can be generated by the density gradient only. It is given by

$$
R_{2}:=\frac{|\mu \boldsymbol{\Delta}|}{\left|\kappa_{F} \boldsymbol{\partial T}\right|} .
$$

Note that $\kappa_{F}=\kappa-c \mu$. Both quantitative measures $R_{1}$ and $R_{2}$ are shown in the right graphs of Figs. 8.2 and 8.3 and will also be discussed below.

\subsubsection{Discussion of Transport Coefficients}

The dotted black lines in Figs. 8.1 show that the two-parameter fit according to Eq. (8.1) with Eqs. (8.6) is very good for all coefficients of restitution $\alpha$ and all global area fractions $\phi_{0}$ presented. In a large region in the middle of each system the fit is hardly distinguishable from the directly measured heat flux. For higher inelasticities $(\alpha \lesssim 0.7-0.8)$ and lower densities $\left(\phi_{0} \lesssim 0.1-0.2\right)$ the fit becomes worse. This is not surprising because in these cases the gradients increase significantly leading to a failure of the gradient expansion, Eq. (8.1).

In the left graph of Figs. 8.2 we show both transport coefficients $\kappa$ (full red lines) and $\mu / \varepsilon$ (full dark green lines) as a function of the coefficient of restitution $\alpha$ for a very wide range of coefficients of restitution $0.2 \leq \alpha \leq 0.999$ for moderately dense systems, $L_{x}=20, L_{y}=25$, $N=256, \phi_{0}=0.4$. The left graph of Figs. 8.3 shows the same observables for $0.5 \leq \alpha \leq$ 0.999 for a total area fractions $\phi_{0}=0.2$. The blue lines show $\kappa_{F}$ from a fit to Fourier's law. The dashed orange and light green lines show the global $\kappa_{J R_{0}}$ and $\mu_{J R_{0}} / \varepsilon$ from Eqs. (8.2).

Both transport coefficients, $\kappa$ and $\mu / \varepsilon$, extracted from our data are non-monotonic in $\alpha$. Starting at inelastic systems and increasing $\alpha$, the thermal conductivity $\kappa$ first decreases, goes through a minimum and then sharply increases again to tend to a non-zero constant in the elastic limit for all densities studied. The other transport coefficient is divided by $\varepsilon$ because we assume $\mu \propto \varepsilon$. Its behavior depends on the density. For moderate densities (Figs. 8.2), coming from inelastic systems, $\mu / \varepsilon$ first increases with increasing $\alpha$, reaches a maximum and finally decreases again. This maximum is in the vicinity of the minimum of $\kappa$ but clearly not at the same value of $\alpha$. For lower density systems (Figs. 8.3), $\mu / \varepsilon$ first decreases when the inelasticity is reduced, reaches a minimum, and sharply increases again. It is not clear whether it will reach a maximum and decrease again or keep increasing and possibly reach a finite value.

The insets show a magnification of the quasi-elastic region and reveal that the measured coefficient of thermal conductivity $\kappa$ is in good agreement with the predictions by Jenkins and Richman [JR85] for $\alpha \geq 0.99$ and moderately dense systems (Figs. 8.2). For higher inelasticities 

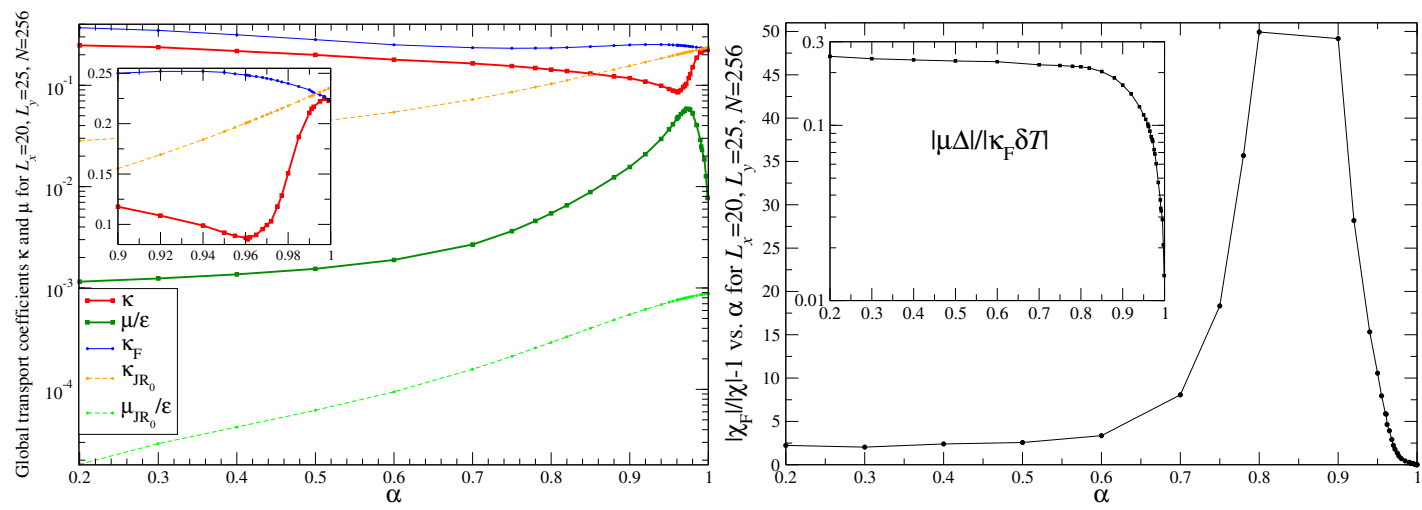

Figure 8.2.: The left graph shows the transport coefficients $\kappa$ and $\mu / \varepsilon$ for systems with $L_{x}=20$, $L_{y}=25$, and $N=256$, corresponding to a line density $\lambda=10.24$ and a total area fraction $\phi_{0}=0.4$. We compare the values obtained from our simulations with the corresponding predictions from Ref. [JR85], Eqs. (8.2), for $0.2 \leq \alpha \leq 0.999$. Note, however, that the theory in Ref. [JR85] assumes quasielastic systems - therefore agreement is not expected for values of $\alpha$ that are not close to one. The inset of the left graph shows a magnification of of the quasi-elastic regime. The right graph quantifies the importance of $\mu$ by showing $R_{1}$ and $R_{2}$ as defined in Eqs. (8.7) and (8.8), and discussed in the text.

significant deviations are observed. Since Jenkins and Richman [JR85] assume quasi-elastic systems we cannot expect their results to be valid for higher inelasticities. Still, their theory yields the correct order of magnitude of the measured $\kappa$ for all $\alpha$. As to $\mu_{J R_{0}}$ the deviations to $\mu$ are quite strong. The discrepancy of $\mu$ (more than one order of magnitude in most cases) is unclear to us. For lower densities (Figs. 8.3) it has not been possible to obtain the transport coefficients for sufficiently elastic systems to judge whether or not the measured data will converge to the predictions by Jenkins and Richman [JR85].

Soto et al. [SMR99] have performed molecular dynamics (MD) simulations to study $\mu$ for a very dilute granular gas on a vibrated plane under gravity. They made use of the non-monotonic behavior of the temperature within such systems to extract $\mu$ at that position where the temperature gradient vanishes - a method that works in the presence of gravity only, even though their results are virtually independent of the magnitude of gravity. This suggests that it should be valid for zero-gravity environments, too. In comparisons we find that their coefficient $\mu$ is always much smaller than ours, up to one order of magnitude. This suggests that the evaluation of $\mu$ is a subtle task and $\mu$ might depend on global system parameters - similarly to the local equation of state in chapter 7 . Other reasons might be that zero-gravity environments behave qualitatively different than gravitational ones and, probably most importantly, the simulations of Ref. [SMR99] apply to very low densities only and not to $0.1 \leq \phi_{0} \leq 0.4$. Furthermore, the difficulties in our fitting procedure increase considerably as $\alpha \rightarrow 1$. In this limit the temperature gradient and the density gradient both become linear in $x$ and thus proportional to each other, see Figs. 6.12 and 6.13 (page 51) and Eqs. (6.4) and (6.5) on page 57. Consequently, it is not possible for $\alpha \rightarrow 1$ to determine two parameters from the fit unambiguously. Finally, we 

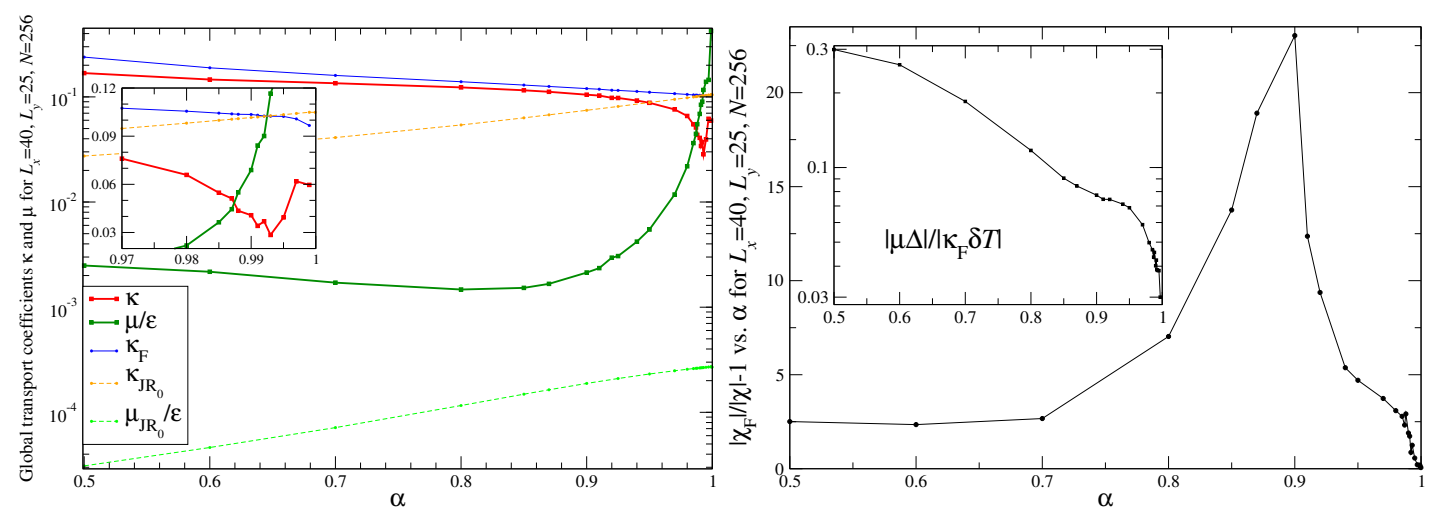

Figure 8.3.: Same as Fig. 8.2 but for systems with $L_{x}=40$, corresponding to a total area fraction of $\phi_{0}=0.2$. Compared to Fig. 8.2 the dip in $\kappa$ is shifted towards elastic systems and $\mu / \varepsilon$ does not decrease again. Of course, the plain $\mu$ still vanishes for $\alpha \rightarrow 1$. The right graphs are qualitatively similar to the ones in Fig. 8.2.

expect the transport coefficients to be $x$-dependent and not constant as assumed in our fitting procedure.

To estimate the degree of inhomogeneity of the transport coefficients in $x$ we have divided the sample box into an inner and an outer part and fitted the heat current using data from either half of the box only. The scattering of the data from the inner and outer part is shown in Fig. 8.4 (indigo and magenta lines, respectively) and provides a rough measure for the effects of inhomogeneous transport. The results for the outer half (magenta lines) are almost identical to the ones for the full system (black lines). This is also true for the inner part (indigo lines) except for the weakly inelastic systems for which the absolute value of the heat flux in the middle of the sample is so small that statistical fluctuations dominate. This shows that the determination of the transport coefficients clearly needs further investigation, especially in the quasi-elastic regime.

Let us now turn to the importance of the new transport coefficient $\mu$. The right graphs of Figs. 8.2 and 8.3 show the quantitative measures $\left(R_{1}\right.$ and $\left.R_{2}\right)$ of the contribution to the heat flux that is not proportional to the temperature gradient. The main graphs show $R_{1}$ as defined in Eq. (8.7) and the insets show $R_{2}$ as defined in Eq. (8.8). From the main graphs as well as from the insets we see that the contribution that is not proportional to the temperature gradient becomes negligible in the elastic limit, as expected. The insets further show that this contribution is never greater than $30 \%$ for all systems studied and that it is more important for larger inelasticities.

The peaks in $R_{1}$ (main graphs) approximately indicate the border line up to which Eq. (8.1) is a good approximation to the measured heat flux. As already mentioned, fits to Fourier's law are hardly distinguishable from the ones to Eq. (8.1) for quasi-elastic systems. For moderately inelastic systems, shown to the right of the peaks in the right graphs of Figs. 8.2 and 8.3, the 


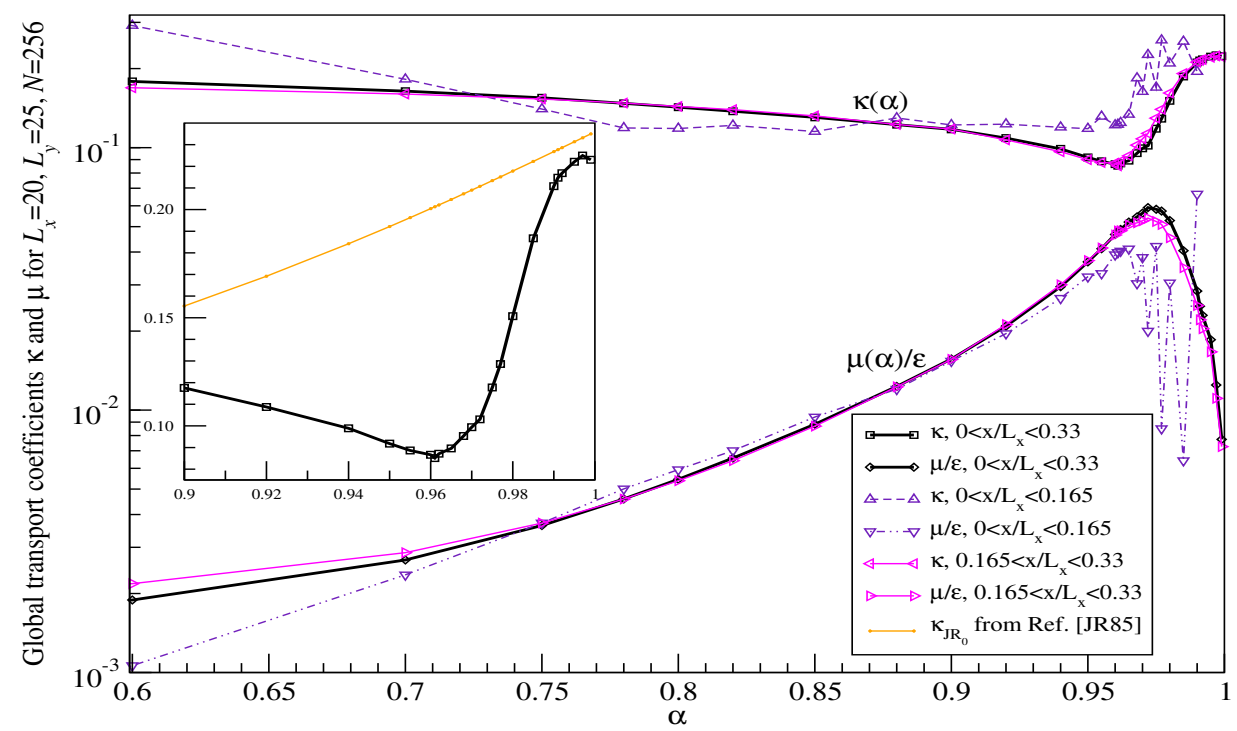

Figure 8.4.: Transport coefficients $\kappa$ and $\mu / \varepsilon$ for the same systems as in Fig. $8.2\left(L_{x}=20, L_{y}=25\right.$, and $N=256$, corresponding to a line density $\lambda=10.24$ and a total area fraction $\phi_{0}=0.4$ ). In addition we show here the values for the transport coefficients obtained for the inner (indigo) or outer part (magenta) only. The inset compares $\kappa$ from our simulations to the value of Ref. [JR85].

two-parameter fit works well while Fourier's law already fails - leading to a high value of $R_{1}$. For even lower inelasticities, shown to the left of the peaks, Eq. (8.1) starts to fail, too, such that neither description is able to faithfully reproduce the directly measured heat flux. This causes $R_{1}$ to decrease. 


\section{Absence of Scaling for Velocity Distributions in Vibrated Systems}

Finally, we examine the local velocity distributions of the driven granular gas in the stationary state. We distinguish between the local distribution

$$
f_{x}\left(x, v_{x}\right):=\frac{1}{\rho(x)} \int_{\mathbb{R}} d v_{y} f_{\text {stat }}\left(x, v_{x}, v_{y}\right)
$$

at position $x$ of the velocity component $v_{x}$ in the direction of the driving and the distribution

$$
f_{y}\left(x, v_{y}\right):=\frac{1}{\rho(x)} \int_{\mathbb{R}} d v_{x} f_{\text {stat }}\left(x, v_{x}, v_{y}\right)
$$

of the velocity component $v_{y}$ perpendicular to the direction of the driving. By definition, these velocity distributions are normalized to unity.

In order to determine $f_{x}$ and $f_{y}$ from the simulation we use two different methods. The first one extracts them directly according to their definitions (9.1) and (9.2) from $f_{\text {stat }}$ in the following way: We count the number of particles $f\left(\boldsymbol{r}, v_{x}, v_{y}, t\right)\left|\mathcal{V}_{\boldsymbol{r}}\right| d v_{x} d v_{y}$ at time $t$ in cell $\mathcal{V}_{\boldsymbol{r}}$ with an $x$ component of the velocity between $v_{x}$ and $v_{x}+d v_{x}$ and $y$-component of the velocity between $v_{y}$ and $v_{y}+d v_{y}$ and average over a long time interval. One disadvantage of this method is that it yields a smeared-out velocity distribution, which is spatially averaged over the width of the strip $\mathcal{V}_{x}$ centered around $x$. This is of practically no importance in the middle of the simulation box, but strongly disturbing for resolving the subtleties which occur close to the driving walls and are presented in Sec. 9.1. The second way of measuring the velocity distributions avoids this problem: To get $f_{i}(i=x$ or $y)$ we keep track of all those particles which pass the line parallel to the $y$-axis at position $x$ within a very long time interval of length $\tau$ and whose $i$ th component of the velocity lies in a small interval of width $\Delta v$ around $v_{i}$. Let us enumerate these particles by $n_{i}$ and denote their velocity components by $v_{x}^{\left(n_{i}\right)}$ and $v_{y}^{\left(n_{i}\right)}$. Then one has (in the limits $\tau \rightarrow \infty$ and $\Delta v \rightarrow 0$ )

$$
\rho(x) f_{i}\left(x, v_{i}\right)=\frac{1}{\tau \Delta v L_{y}} \sum_{n_{i}} \frac{1}{\left|v_{x}^{\left(n_{i}\right)}\right|},
$$

because for $i=x$, resp. $i=y$, the right-hand side of (9.3) is equal to

$$
\int_{\mathbb{R}} d v_{y} \frac{\left|F_{x}\left(x, v_{x}, v_{y}\right)\right|}{\left|v_{x}\right|} \quad \operatorname{resp.} \int_{\mathbb{R}} d v_{x} \frac{\left|F_{x}\left(x, v_{x}, v_{y}\right)\right|}{\left|v_{x}\right|} .
$$


Here, $F_{x}\left(x, v_{x}, v_{y}\right):=v_{x} f_{\text {stat }}\left(x, v_{x}, v_{y}\right)$ denotes the $x$-component of the (differential) current density in the stationary state at position $x$ of particles with velocity components $v_{x}$ and $v_{y}$.

As compared to the first method of measuring velocity distributions, this one is also statistically more effective for determining rare events, such as the high-velocity tails in Sec. 9.2. However, as far as $f_{x}\left(x, v_{x}\right)$ is concerned for small $v_{x}$, the second method is inferior to the first, because (9.3) assigns a large weight to the relevant events and therefore amplifies statistical fluctuations, too.

\subsection{Effects of the Discontinuity at a Driving Wall}

Particle-number conservation at a driving wall requires the incoming particle flux at the wall to be equal to the outgoing flux. For the velocity distribution $f_{x}$ this implies the boundary condition [BRMM00]

$$
f_{x}\left(\mp L_{x} / 2, v_{x}\right)=\Theta\left( \pm v_{x}-v_{\mathrm{dr}}\right)\left(1 \mp \frac{v_{\mathrm{dr}}}{v_{x}}\right) f_{x}\left(\mp L_{x} / 2,-v_{x}+v_{\mathrm{dr}}\right),
$$

which must hold for all $v_{x}>0$. Here $\Theta(z):=1$ for $z \geq 0$, respectively $\Theta(z):=0$ for $z<0$, denotes Heaviside's unit-step function and $v_{\mathrm{dr}}=1$ in our units chosen. (Note that we use a driving velocity $v_{\mathrm{dr}}=v_{0}$ here instead of $v_{\mathrm{dr}}=\varepsilon v_{0}$ as used in chapter 6). The boundary condition (9.5) relates the distribution $f_{x}$ of velocities prior to a collision with the wall to the one after a collision with the wall. In contrast, the velocity distribution $f_{y}$ must obey the usual reflection symmetry in $v_{y}$ everywhere in the system, that is

$$
f_{y}\left(x, v_{y}\right)=f_{y}\left(x,-v_{y}\right)
$$

for all $|x| \leq L_{x}$ and all $v_{y}>0$.

We measured the velocity distribution $f_{x}\left(x, v_{x}\right)$ at 25 different positions in a moderately inelastic system with coefficient of restitution $\alpha=0.9$. Fig. 9.1 shows the rescaled velocity distribution $\tilde{f}_{x}\left(x, v_{x} / \sqrt{T_{x}(x)}\right):=f_{x}\left(x, v_{x}\right) \sqrt{T_{x}(x)}$, measured using method one. At the driving walls $\tilde{f}_{x}$ is seen to obey the boundary condition (9.5). When moving from a driving wall towards the center of the system, the gap in $\tilde{f}_{x}$ gets gradually smeared out, and $\tilde{f}_{x}$ becomes more and more symmetric. Clearly, even for this moderately inelastic system $\tilde{f}_{x}$ does not scale for different $x$. The two extreme cases for $x=-L_{x} / 2$ and $x=0$ are shown together in Fig. 9.2. The data for $\tilde{f}_{x}$ at the wall was obtained with the second method for measuring velocity distributions. This result is in agreement with Direct-Simulation-Monte-Carlo results in Ref. [BRMM00] and Molecular-Dynamics simulations in Ref. [BT02b]. 


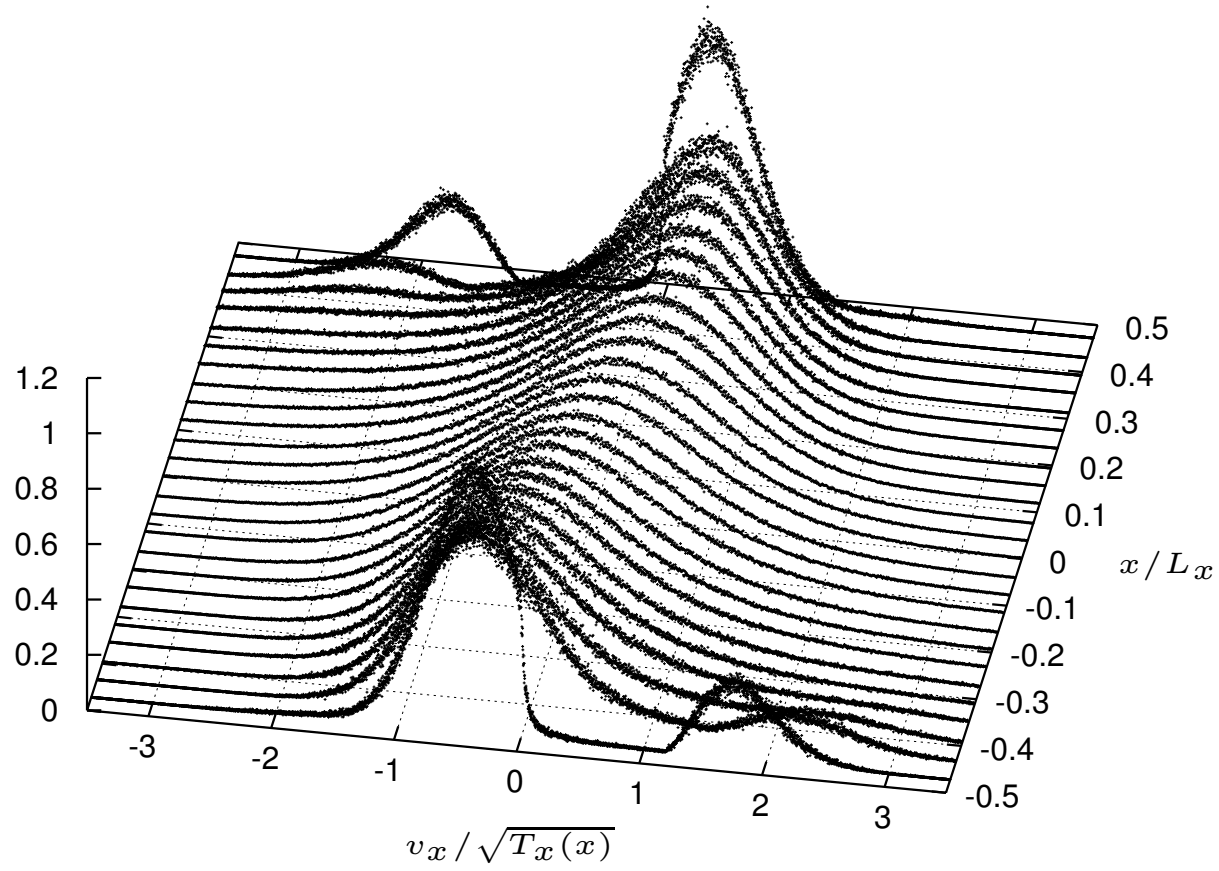

Figure 9.1.: Rescaled velocity distributions $\tilde{f}_{x}$ for different $x$. [System parameters: $\alpha=0.9, L_{x}=20$, $\left.\lambda=10.24\left(N=256, \phi_{0}=0.4\right)\right]$

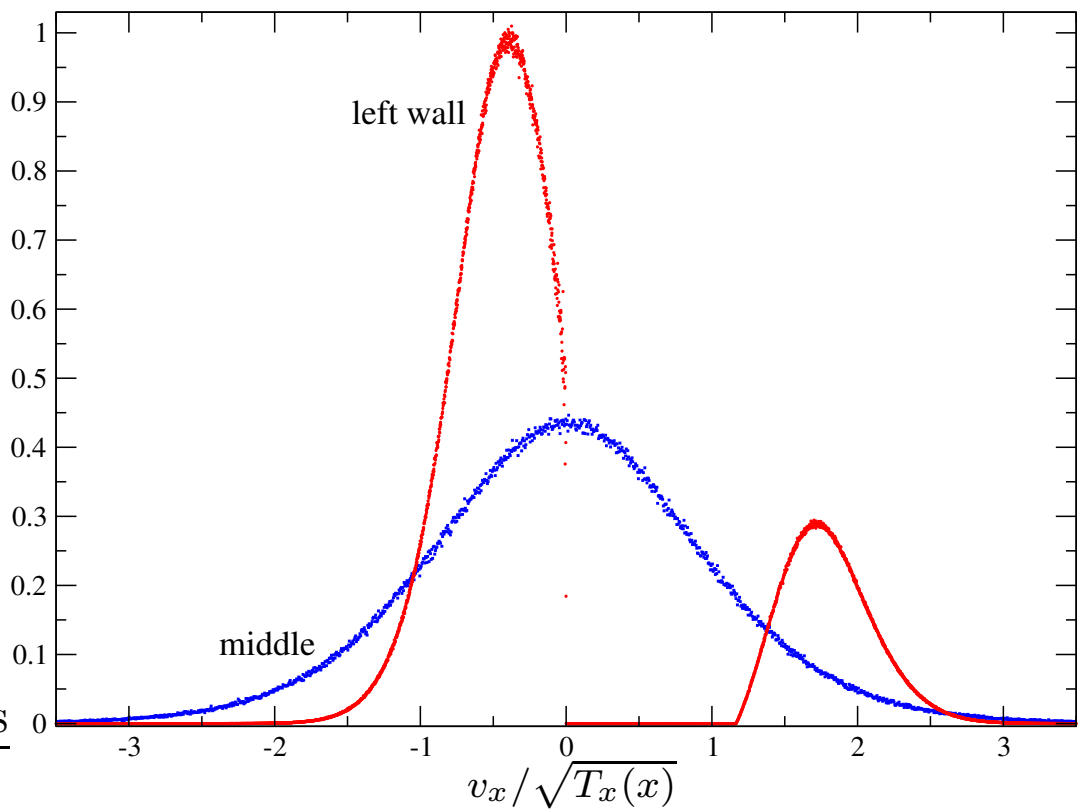

Figure 9.2.: Two rescaled velocity distributions $\tilde{f}_{x}$ from Fig. 9.1: at the left wall and in the middle of the system. 


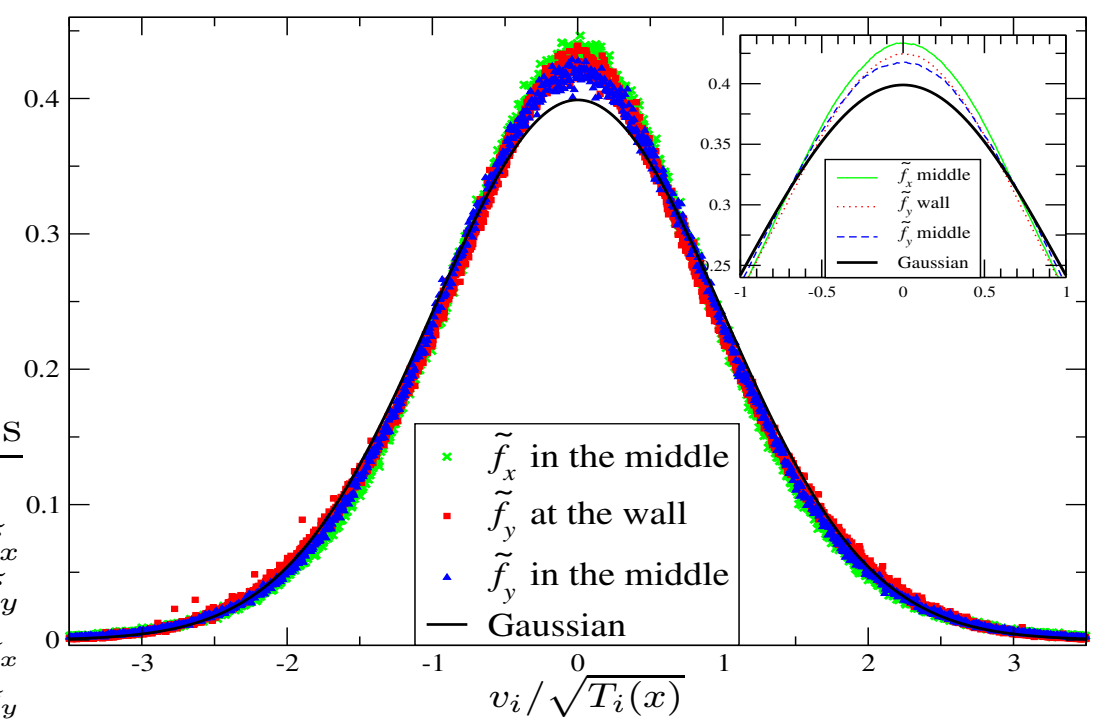

Figure 9.3.: Combined plot of rescaled velocity distributions of the same system as in Figs. 9.1 and 9.2: $\tilde{f}_{x}$ in the middle of the sample (green crosses), $\tilde{f}_{y}$ at a driving wall (red squares), and $\tilde{f}_{y}$ in the middle (blue triangles). Also shown is a centered Gaussian with unit variance (black solid line). The inset shows the central part of the main graph with the (1433/2964/1340) data points being smoothed (running average over (41/85/38) data points).

\subsection{Non-Universal High-Velocity Tails}

Our main result for the velocity distributions is the non-scaling and multiformity of their tails. In order to observe these phenomena, extensive simulations for capturing rare events are required and the data have to be analyzed on a logarithmic scale. In contrast, on a linear scale the rescaled velocity distributions seem to collapse approximately, as was observed previously by e.g. [GZBN97, MSS04]. As an example we show in Fig. 9.3 the rescaled distributions $\tilde{f}_{y}\left(x, v_{y} / \sqrt{T_{y}(x)}\right):=f_{y}\left(x, v_{y}\right) \sqrt{T_{y}(x)}$ in the middle of the sample $(x=0)$ and at a driving wall $\left(x= \pm L_{x} / 2\right)$ for the same moderately inelastic system $(\alpha=0.9)$ as in Figs. 9.1 and 9.2. Clearly, the curves are reflection-symmetric in $v_{y}$. For comparison, we have also included the symmetric distribution $\tilde{f}_{x}$ in the middle of the system. Deviations are visible for small velocities only, as the inset of Fig. 9.3 shows. The corresponding Gaussian (solid line) in Fig. 9.3 suggests that the velocity distributions are close to but not identical to a Maxwellian. The approximate data collapse, observed on this level of accuracy in Fig. 9.3, even continues to hold if the coefficient of restitution of the gas is varied in a not too large extent. In considerably more inelastic systems, such as for $\alpha=0.5$, this is not true any longer. For example, the peak of $\tilde{f}_{x}$ measured in the middle of the system would be considerably broader and flatter than the ones of $\tilde{f}_{y}$ in the center and at the wall (not shown). 

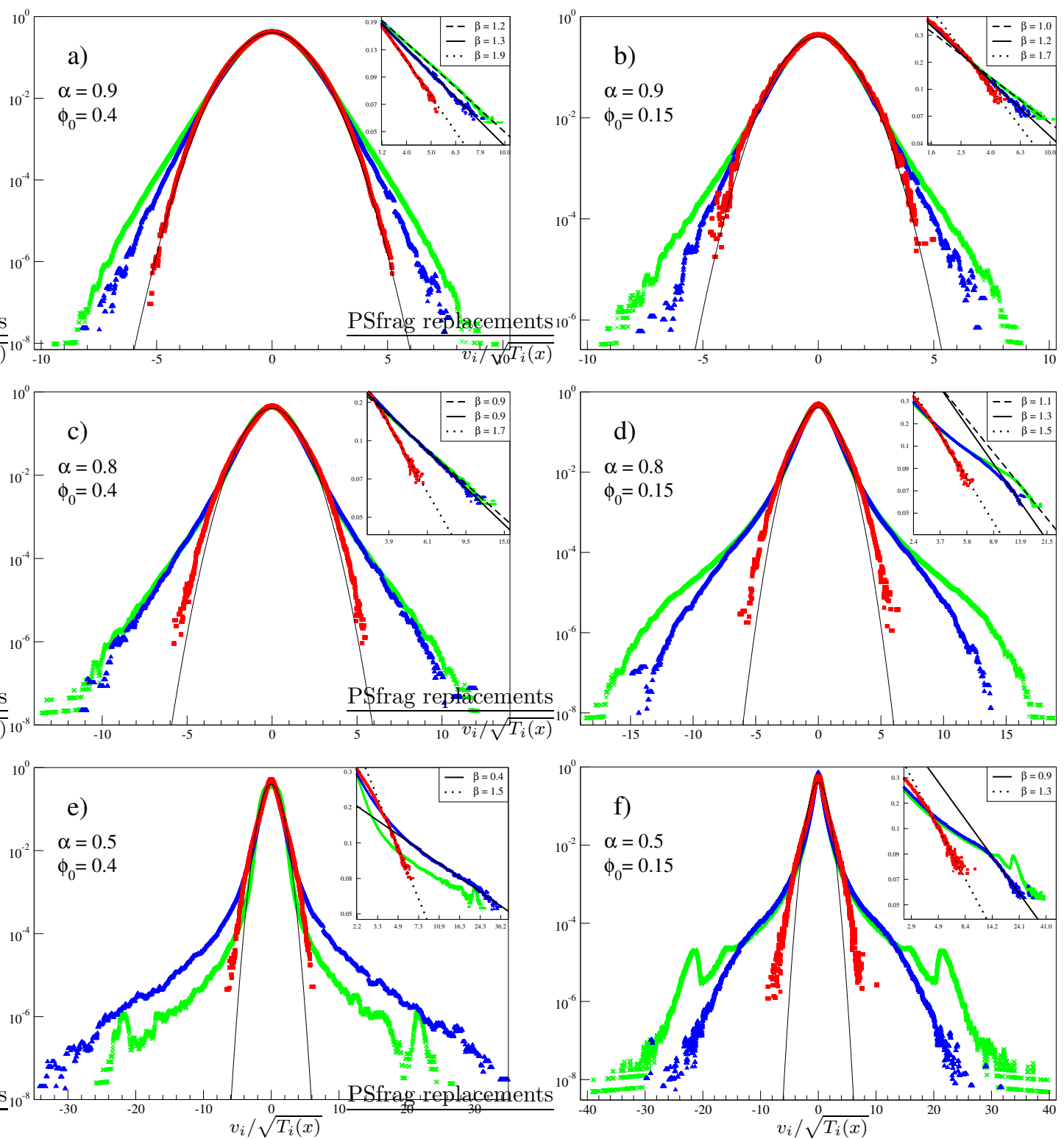

Figure 9.4.: Rescaled velocity distributions $\tilde{f}_{x}$ in the middle of the sample (green crosses), $\tilde{f}_{y}$ at a driving wall (red squares), and $\tilde{f}_{y}$ in the middle (blue triangles) as in Fig. 9.3, but here smoothed data are shown on a semi-logarithmic scale and velocities of much higher absolute values are included. The solid line is a centered Gaussian with unit variance. Different parts of the figure represent different systems with decreasing global area fraction $\phi_{0}$ (left to right) and decreasing coefficient of restitution $\alpha$ (top to bottom). The remaining system data are $L_{x}=20, \lambda=10.24(N=256)$ for the left column and $L_{x}=50, \lambda=9.6(N=240)$ for the right column. The insets show $\left|\ln \tilde{f}_{i}\right|^{-1}$ on a double-logarithmic scale to determine the decay exponent $\beta$ from (9.7). The dashed line is the best linear fit to $\tilde{f}_{x}$ in the middle of the sample, the solid line to $\tilde{f}_{y}$ in the middle, and the dotted line to $\tilde{f}_{y}$ at the wall. Part a) corresponds to the system of Fig. 9.3. 
In contrast, Fig. 9.4 a) shows the same data of Fig. 9.3 on a semi-logarithmic scale and includes also velocities of much higher absolute values. From this figure it is evident that scaling does not hold in the high-velocity tails of the distributions either. Similar observations were made before in e.g. [BT02b, vZM04, BRM03]. The type of decay in the high-velocity tails is different for $\tilde{f}_{x}$ and $\tilde{f}_{y}$, and also depends on the position in the sample, the coefficient of restitution $\alpha$, and the global area fraction $\phi_{0}$. This is illustrated by examples of different systems in Fig. 9.4. In the insets we show $\left|\ln \tilde{f}_{i}\right|^{-1}$ on a double-logarithmic scale in order to determine the decay exponent $\beta$ defined by

$$
\ln \tilde{f}_{y}\left(0, v_{y} / \sqrt{T_{y}(0)}\right) \stackrel{\left|v_{y}\right| \rightarrow \infty}{\sim}-\left|v_{y} / \sqrt{T_{y}(0)}\right|^{\beta}
$$

for $\tilde{f}_{y}$ in the middle of the sample. The exponent is defined accordingly for $\tilde{f}_{y}$ at the wall and $\tilde{f}_{x}$ in the middle of the sample. For the moderately inelastic systems with $\alpha=0.9$ in the first row of Fig. 9.4, the asymptotics has been clearly reached. We note that $\beta$ is different for the different distributions, and also depends on the global area fraction $\phi_{0}$. Upon lowering $\alpha$ (top to bottom in Fig. 9.4) and/or decreasing the global area fraction $\phi_{0}$ (left to right), the tails of $\tilde{f}_{y}$ get more and more populated, that is, $\beta$ decreases. In very simple terms, this may be understood from the fact that (i) the largest typical velocities are always of the order of $v_{\mathrm{dr}}=1$ (see also Fig. 9.5) and (ii) that $v_{\mathrm{dr}} / \sqrt{T_{i}(x)}$ increases up to 20 with decreasing $\alpha$ and decreasing $\phi_{0}$. Hence, a Maxwellian velocity distribution would not be able to supply enough probability to particles with velocities of the order of $v_{\mathrm{dr}}$, instead higher-populated tails are needed. This argument suggests different behavior in different velocity regions so that the distributions cannot be fitted to the functional form (9.7) over the entire range of velocities [vZM04]. Indeed, such a behavior can be seen in Fig. 9.4 d), e) and f). The final asymptotics could not always be deduced from the simulations, even though our data include velocities which are up to 40 times bigger than the appropriate granular velocities $\sqrt{T_{i}}$. This applies to $\tilde{f}_{y}$ in the middle of the sample in part e), where we suspect that the final asymptotics has not been reached. An asymptotic analysis of $\tilde{f}_{x}$ in the middle of the sample is even more problematic due to particles that reach the system center from a driving wall without undergoing a collision. These particles give rise to the side peaks of $\tilde{f}_{x}$ in parts e) and f), which have prevented us from determining $\beta$ in these cases.

Fig. 9.4 contains smoothed data as in the inset of Fig. 9.3, and the scales of the horizontal axes are determined by the square root of the appropriate granular temperatures. For comparison, Fig. 9.5 shows the unsmoothed data corresponding to Fig. $9.4 \mathrm{f}$ ), plotted directly versus $v_{i}$ (in units of $v_{\mathrm{dr}}$ ). The side peaks of $\sqrt{T_{x}(0)} f_{x}\left(0, v_{x}\right)$ in the middle of the sample (green crosses in Fig. 9.5) are due to the above-mentioned particles which fly from a driving wall to the system center without undergoing a collision. Concerning the distribution of the $y$-component of the velocity in the middle of the sample (blue triangles in Fig. 9.5) we find for the vast majority of particles that $\left|v_{y}\right|$ is less than $80 \%$ of the driving velocity. It is in the region of this highest velocity observed for $\sqrt{T_{y}(0)} f_{y}\left(0, v_{y}\right)$ in the middle of the sample (blue triangles), where $\sqrt{T_{x}(0)} f_{x}\left(0, v_{x}\right)$ in the middle of the sample (green crosses) changes abruptly its slope. 


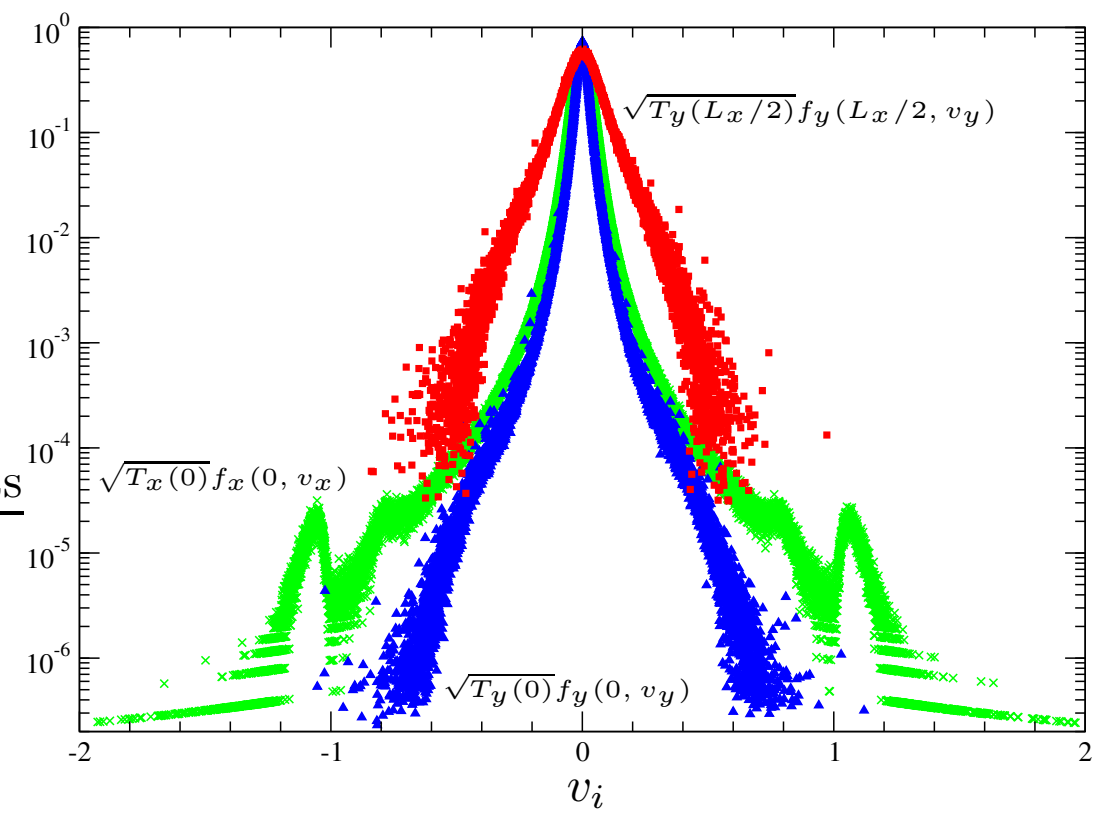

Figure 9.5.: Velocity distributions for the system in Fig. 9.4 f), but with unsmoothed data and without rescaling of the horizontal axis.

The exponent $\beta$ has also been determined experimentally in a strongly driven gas so that gravity effects are small [RM00]. A value $\beta \approx 1.55$ was measured for a gas with coefficient of restitution $\alpha \approx 0.93$. It was found to be remarkably independent of the global area fraction $\phi_{0}$, which was varied from 0.05 to 0.3 . We have also simulated the system of [RM00] with zero gravity (not shown) and reproduced their value for $\beta$. Even though the experimental data cover a wide range of velocities, some of the interesting phenomena discussed in Fig. 9.4 cannot be observed in this range. For the same reason the significance of the particular value $\beta \approx 1.55$ should not be overestimated.

Simulations of a driven granular gas in a circularly shaped box also yield stretched Gaussian tails [vZM04]. In addition, evidence is given that $\beta$ depends only on the coefficient of restitution and the average ratio of the number of particle-wall collisions and particle-particle collisions. For homogeneously driven systems the tail of the velocity distribution was theoretically predicted [vNE98] to be governed by the decay exponent $\beta=3 / 2$. Simulations of homogeneously driven systems [MSS01, BT03] observed the exponent $\beta=3 / 2$ only for unrealistically low values of the coefficient of restitution. 


\section{Homogeneously Driven Systems of Spheres with Coulomb Friction}

Incorporating Coulomb friction turns out to be quite challenging. For this reason we restrict our analysis of frictional particles to homogeneously driven systems. The driving mechanism has been specified in Sec. 2.2, see page 12. Binary collisions with Coulomb friction are modeled using the Walton model, cf. Sec. 2.1.2, page 10.

In a homogeneously driven system, the long-time averages of all hydrodynamic fields are homogeneous. Instead it is interesting to study the mean translational and rotational temperatures, their stationary ratio, and their relaxation to the steady state. In this chapter we will present and discuss an analytic mean field calculation to obtain differential equations for the full time evolution of the translational and rotational temperature. Technical details of the calculation can be found in appendix $\mathrm{C}$.

Since the analytic calculations are very elaborate we will introduce and probe various simplifications to the collision rules for Coulomb friction (the Walton model, Eq. (2.8)). These simplifications (labeled models B through D) will be introduced in Sec. 10.2. Model A is the well-known model of constant tangential restitution, model $\mathrm{E}$ is the full Walton model. All these models can be expressed in terms of effective coefficients of tangential restitution. Using these effective coefficients, we will analytically calculate the differential equations for the time evolution of the translational and rotational energies and compare the results to the one obtained using the full Walton model. In Sec. 10.3 we discuss the steady state, first analytically and then compare the results to Event-Driven simulations by Stefan Luding [ $\left.\mathrm{HCZ}^{+} 05\right]$. The algorithm of the simulations used in this chapter is very similar to the one used for vibrated systems [Lub91]. In Sec. 10.4 we turn our attention to the approach to the steady state before we summarize the results for homogeneously driven systems with Coulomb friction in Sec. 10.5 .

One way of simplifying the collision rules is to consider the tangential restitution averaged over all impact angles $\gamma$. This reduces the problem to one with a constant coefficient of tangential restitution that depends on the three parameters of the Walton model, i.e. the coefficient of normal restitution, the coefficient of Coulomb friction, and the coefficient of tangential restitution for sticking contacts. For this purpose we need to know the probability distribution of impact angles as discussed in the next section. 


\subsection{Impact-Angle Probability Distribution}

The assumption of "molecular chaos" implies a homogeneous distribution of the collision parameter $b=a \sin \gamma_{12}$ which is simply related to the angle $\gamma_{12}$ between the relative translational velocity $\boldsymbol{v}_{12}$ and the contact normal $\hat{\boldsymbol{n}}$ according to $\cos \gamma_{12}=\boldsymbol{v}_{12} \cdot \hat{\boldsymbol{n}} /\left|\boldsymbol{v}_{12}\right|$, see Fig. 2.1. Hence the probability distribution of $\sin \gamma_{12}$ is constant, $P_{12}^{\prime}\left(\sin \gamma_{12}\right) \equiv 1$. (The "prime" indicates probability functions of the sine or the cosine of the angle.) A uniform probability $P_{12}^{\prime}$ implies that the distribution of the angle $P_{12}\left(\gamma_{12}\right)=-\cos \gamma_{12}$, so that grazing contacts appear less probable than central collisions when a fixed interval $d \gamma_{12}$ is considered. The uniform $P_{12}^{\prime}\left(\sin \gamma_{12}\right)$ is in agreement with our numerical data for very smooth particles, see full lines $\left[P_{12}(\gamma) \equiv P_{12}^{\prime}(\sin \gamma)\right]$ and triangles [simulations of very smooth particles] in Fig. 10.1. For rougher particles $P_{12}(\gamma)$ is not a good approximation to the simulational data. Instead, the real $P(\gamma)$ must be used as will be discussed below.

In general, the impact angle $\gamma$ between the relative velocity of the contact point $\boldsymbol{g}$ and the contact normal $\hat{\boldsymbol{n}}$ is different from the angle $\gamma_{12}$ between the relative translational velocity $\boldsymbol{v}_{12}$ and the contact normal $\hat{\boldsymbol{n}}$, as displayed in Fig. 2.1. The two angles are identical only in the case of smooth particles or in the limit of vanishingly small rotational velocities. In the general case we compute $P^{\prime}(\cos \gamma)$ by averaging over all binary collisions

$$
P^{\prime}(\cos \gamma)=\left\langle\delta\left(\cos \gamma-\frac{\boldsymbol{g} \cdot \hat{\boldsymbol{n}}}{|\boldsymbol{g}|}\right)\right\rangle_{\text {coll }} .
$$

This average can only be computed approximately. We assume that the translational and rotational velocities of the colliding particles are distributed according to Gaussians with a temperature $T_{\mathrm{tr}}$ for the translational and a temperature $T_{\text {rot }}$ for the rotational velocities. Within this approximation the above average is given explicitly by

$$
P^{\prime}(\cos \gamma)=\frac{J\left(\delta\left(\cos \gamma-\frac{\boldsymbol{g} \cdot \hat{\boldsymbol{n}}}{|\boldsymbol{g}|}\right)\right)}{J(1)}
$$

with the phase space integral

$$
J(X)=\int d \Gamma_{1} d \Gamma_{2}\left(\boldsymbol{v}_{12} \cdot \hat{\boldsymbol{n}}\right) \Theta\left(-\boldsymbol{v}_{12} \cdot \hat{\boldsymbol{n}}\right) \delta\left(\left|\boldsymbol{r}_{12}\right|-2 a\right) X,
$$

where $X=X\left(\Gamma_{1}, \Gamma_{2}\right)$, and the phase space element

$$
d \Gamma_{k}=d^{2} r_{k} d^{2} v_{k} d \omega_{k} e^{-m v_{k}^{2} /\left(2 T_{\mathrm{tr}}\right)} e^{-I w_{k}^{2} /\left(2 T_{\mathrm{rot}}\right)}
$$

for $k=1,2$.

The remaining integrals can be computed analytically, yielding the following expression for the impact angle distribution

$$
P(\gamma)=-\frac{(1+R / q) \cos \gamma}{\left(1+[R / q] \cos ^{2} \gamma\right)^{3 / 2}}
$$



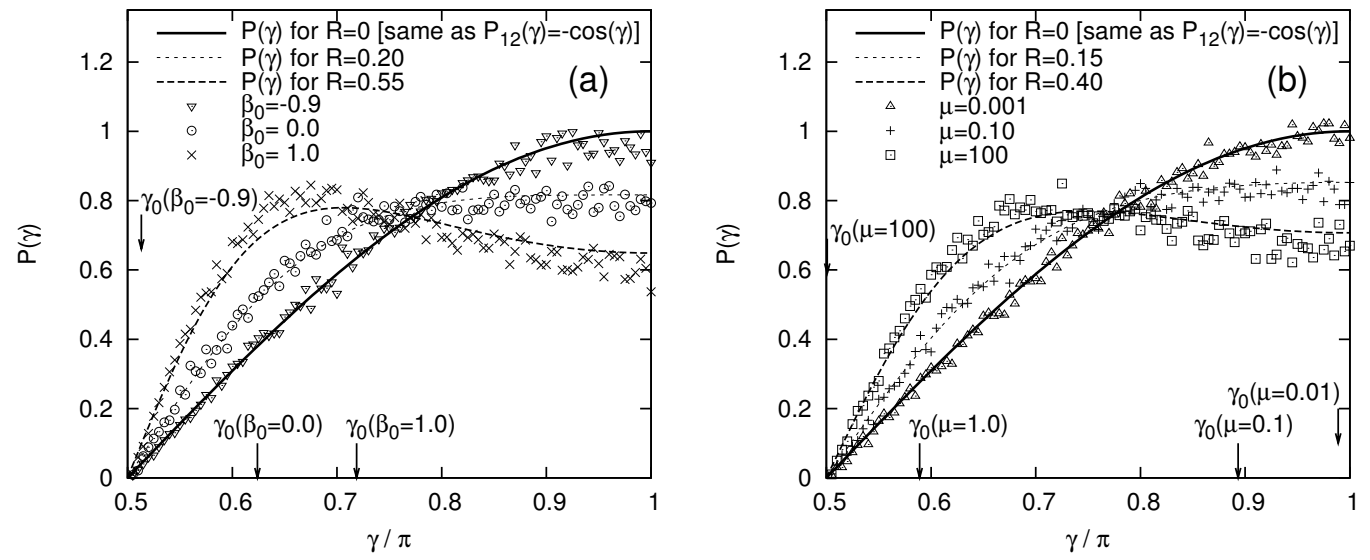

Figure 10.1.: Plots of the probability distribution of $\gamma$ from simulations (symbols) and from Eq. (10.3) with $R$ values from the simulations. The arrows indicate the corresponding $\gamma_{0}$. The parameters are (a) $\alpha=0.95, \mu=0.5$ and variable $\beta_{0}$, and (b) $\alpha=0.95, \beta_{0}=0.4$ and variable $\mu$. Reasonable agreement is observed between the simulations and $P(\gamma)$ as given in Eq. (10.3).

Here we have introduced the ratio of rotational and translational temperatures $R:=T_{\text {rot }} / T_{\text {tr }}$ and recall $q=I /\left(m a^{2}\right)$. The probability distribution $P(\gamma)$ is compared to the results of our simulations in Fig. 10.1. Reasonably good agreement is observed. With increasing rotational temperature, grazing contacts (small $\gamma$ ) become more and more frequent due to the increasing rotational contribution. On the other hand, head-on collisions (large $\gamma$ ) become less probable.

\subsection{Differential Equations for the Time Evolution of Temperatures}

In the following we present different approximations for frictional particles, referred to as models A-E. For every model we calculate differential equations for the time evolution of the translational and rotational temperatures in mean field theory. Model A is the well known model using constant coefficients of normal and tangential restitution, cf. e.g., Refs. [JR85, HZ97]. Model E implements Coulomb friction as introduced by Walton [WB86]. While model A is the mean field solution for rough particles with a constant coefficient of tangential restitution, model $\mathrm{E}$ is the mean field solution for particles with Coulomb friction. Models B through D are approximations to model $\mathrm{E}$ that may be simpler to deal with but have significant shortcomings.

The starting point of our mean-field approach is the theory of Ref. [HZ97] for a freely cooling gas of rough particles with a constant coefficient of tangential restitution ( $\beta=$ const., corresponding to the limit $\mu \rightarrow \infty$ ). The theory is based on a pseudo-Liouville-operator formalism and on the assumption of (i) a homogeneous state, (ii) independent Gaussian probability dis- 
tributions of all degrees of freedom, i.e. all components of the translational and the rotational velocities, and (iii) the assumption of "molecular chaos", i.e. subsequent collisions are uncorrelated. The agreement with simulations is very good as long as the above assumptions are valid [LHMZ98].

The main outcome of this approach is a set of coupled time evolution equations for the translational and rotational MF temperatures $T_{\mathrm{tr}}$ and $T_{\text {rot }}$ [HZ97] which can be extended to also describe arbitrary energy input (driving) [CLH00, CLH02, Lud03]. Given the random driving temperature $T_{\mathrm{dr}}$ and an energy input rate $f_{\mathrm{dr}}$, as defined above, one just has to add the positive rate of change of translational energy $H_{\mathrm{dr}}$, see Eq. (2.13), to the system of equations [CLH00].

\subsubsection{Model A: Constant Coefficient of Tangential Restitution $\beta=\beta_{0}$}

We recall the results of the mean field theory for the model with a constant coefficient of tangential restitution which is obtained from the general case in the limit $\mu \rightarrow \infty$ (see Eqs. (14) in Ref. [LHMZ98]). The system of coupled equations in 2D reads:

$$
\begin{array}{ll}
\frac{d}{d t} T_{\mathrm{tr}}(t) & =H_{\mathrm{dr}}+G\left[-A T_{\mathrm{tr}}^{3 / 2}+B T_{\mathrm{tr}}^{1 / 2} T_{\mathrm{rot}}\right] \\
\frac{d}{d t} T_{\mathrm{rot}}(t) & =\quad 2 G\left[B^{\prime} T_{\mathrm{tr}}^{3 / 2}-C T_{\mathrm{tr}}^{1 / 2} T_{\mathrm{rot}}\right],
\end{array}
$$

Note the choice of signs which lead to positive coefficients. Based on more physical arguments, coefficient $A$ quantifies the dissipation of translational energy, $B$ and $B^{\prime}$ correspond to the interchange of energy between the translational and rotational degrees of freedom, and $C$ describes the dissipation of rotational energy. The coefficient $G$ sets the time-scale of the system, i.e. the collision rate (per particle) $\tau^{-1}=(1 / 2) G T_{\mathrm{tr}}^{1 / 2}$, with

$$
G=\frac{16}{a \sqrt{\pi m}} \phi \chi
$$

Here $\chi$ denotes the pair correlation function at contact. In the approximation proposed by Henderson [Hen75, Hen77, VL82, JR85, SK99], $\chi=(1-7 \phi / 16) /(1-\phi)^{2}$, it depends only on the area fraction of the granular gas $\phi=\pi a^{2} N / V$. The four constants $A, B, B^{\prime}$ and $C$ read

$$
\begin{aligned}
A=A_{r}+A_{\eta_{0}}, \quad A_{r} & :=\frac{1-\alpha^{2}}{4}, \\
A_{\eta_{0}} & :=\frac{\eta_{0}}{2}\left(1-\eta_{0}\right), \\
B^{\prime}=B=B_{\eta_{0}} & :=\frac{\eta_{0}^{2}}{2 q}, \text { and } \\
C=C_{\eta_{0}} & :=\frac{\eta_{0}}{2 q}\left(1-\frac{\eta_{0}}{q}\right) .
\end{aligned}
$$

where

$$
\eta_{0}:=\eta\left(\beta_{0}\right)=\frac{q\left(1+\beta_{0}\right)}{2(q+1)},
$$

with $\eta(\beta)$ being defined in Eq. (2.6). 


\subsubsection{Model B: Averaged Simplified Coulomb Friction $\rightarrow \beta=\left\langle\beta\left(\gamma_{12}\right)\right\rangle_{\gamma_{12}}$}

A first step beyond the above theory with a constant $\eta_{0}=\eta\left(\beta_{0}\right)$, is the replacement of $\beta(\gamma)$ by its average

$$
\langle\beta\rangle=\int_{\pi / 2}^{\pi} d \gamma P(\gamma) \beta(\gamma)
$$

The integral over $\gamma$ from $\pi / 2$ to $\pi$ has to be split into two parts, one corresponding to the range $\pi / 2<\gamma<\gamma_{0}$ for which there is Coulomb sliding and $\beta$ is given by Eq. (2.8), and a second part corresponding to the range $\gamma_{0} \leq \gamma \leq \pi$, for which there is sticking with constant $\beta=\beta_{0}$ (see Fig. 2.2). The critical angle $\gamma_{0}$ is given by $c$, cf. Eq. (2.9).

To simplify the computation, we use the approximation $P(\gamma) \approx P_{12}(\gamma)=-\cos (\gamma)$, such that ${ }^{1}$

$$
\langle\beta\rangle_{12}=-1+\frac{q+1}{q}(1+\alpha) \mu \ln (c+f) .
$$

with the abbreviation

$$
f:=\sqrt{1+c^{2}} .
$$

The averaged coefficient of tangential restitution $\langle\beta\rangle_{12}$ must be inserted into $\eta$ in Eq. (2.6). Thus we obtain the same set of coefficients as in Eqs. (10.6)-(10.9) with $\eta_{0}$ replaced by

$$
\eta_{1}:=\eta\left(\langle\beta\rangle_{12}\right)=\frac{\eta_{0}}{c} \ln (c+f) .
$$

In this approach, only the average value of $\beta$ is considered and fluctuations of $\beta$ with $\gamma$ are neglected. Furthermore the difference between $\gamma$ and $\gamma_{12}$ has been ignored in the averaging procedure. In contrast to model A this is the simplest model to incorporate an approximate Coulomb friction via the coefficient of Coulomb friction $\mu$ in $\langle\beta\rangle_{12}=\langle\beta\rangle_{12}(\mu)$.

\subsubsection{Model C: Averaged Real Coulomb Friction $\rightarrow \beta=\langle\beta(\gamma)\rangle_{\gamma}(R)$}

In model $\mathrm{C}$ we again replace $\beta(\gamma)$ by its average but use the correct impact angle probabilty distribution function $P(\gamma)$ from Eq. (10.3) in the averaging procedure. The result ${ }^{2}$ is an $R$ dependent averaged coefficient of tangential restitution

$$
\langle\beta\rangle(R)=-1+\frac{q+1}{q} \frac{1+\alpha}{4} \frac{\mu}{x} \ln \left[\frac{\frac{R}{q}(f-c)^{2}\left(x \tilde{f}-f+c \frac{R}{q}\right)}{\left(x \tilde{f}-f-c \frac{R}{q}\right)^{2}\left(x \tilde{f}+f-c \frac{R}{q}\right)}\right]
$$

\footnotetext{
${ }^{1}$ simplifying the result we have used the identity $\left(1-\cos \gamma_{0}\right) /\left(1+\cos \gamma_{0}\right)=(c+f)^{2}$

${ }^{2}$ obtained using Maple
} 
with

$$
\begin{aligned}
x^{2} & \equiv x^{2}(R):=1+R / q, \\
\tilde{f} & \equiv \tilde{f}(R):=\sqrt{1+x^{2} c^{2}}
\end{aligned}
$$

and $f$ defined in eq.(10.13). Note that $x$ is an implicit function of time through $R$. For $R \rightarrow 0$ (i.e. $x \rightarrow 1)$ Eq. (10.15) reduces to Eq. (10.12) - as expected. For $R \rightarrow \infty(x \rightarrow \infty)$ there is no friction and $\langle\beta\rangle(R) \rightarrow-1$.

We formally get the same differential equations (10.4) but with non-constant coefficients $A=$ $A(R), B^{\prime}=B=B(R)$, and $C=C(R)$ which are obtained by replacing $\eta_{0}$ by $\eta(\langle\beta\rangle(R))$ in Eqs. (10.6)-(10.9). These coefficients are implicitly time dependent.

\section{Constant tangential restitution limit}

In the limit $\mu \rightarrow \infty, c \rightarrow 0$. In that case model $\mathrm{C}$ reduces to model $\mathrm{A}$.

\section{Weak friction limit}

For $\mu \rightarrow 0, c \rightarrow \infty$ we recover smooth spheres with $\langle\beta\rangle \rightarrow-1$. A series expansion to lowest order in $\mu$ (equivalent to lowest order in $c^{-1}$ ) of Eq. (10.15) reads

$$
\langle\beta\rangle(R)=-1+\frac{q+1}{q}(1+\alpha) \frac{\mu}{x}\left\{|\ln (\mu)|+\ln (x)+\ln \left(\frac{2 \eta_{0}}{1+r}\right)\right\}+\mathcal{O}\left(\mu^{3}\right),
$$

expressed in terms of $x$ and $\mu$.

As long as $x$ stays finite (which is the case for a driven system) the leading order is thus $\mu|\ln (\mu)|$ for small $\mu$. For $x \rightarrow 1$, Eq. (10.18) yields the same result as Eq. (10.12) in leading order in $c^{-1}$.

\section{Comparison of model B and model C}

Due to the implicit nature of model $\mathrm{C}$ it is rather difficult to work out its predictions, e.g., for the ratio of temperatures. Therefore, we present here the mean tangential restitution from models A, B, and C in Fig. 10.2. Note that $\langle\beta\rangle$ for model $\mathrm{C}$ depends not only explicitly on $\mu$ but also implicitly through $R$. To keep the discussion simple, we present results only for some constant, representative values of $R$. The mean restitution for large $R$ is smaller (or equivalently, the corresponding $\mu$ is larger) than for small $R$. Models B and $\mathrm{C}$ become indistinguishable in the limit $R \rightarrow 0$, as expected. 


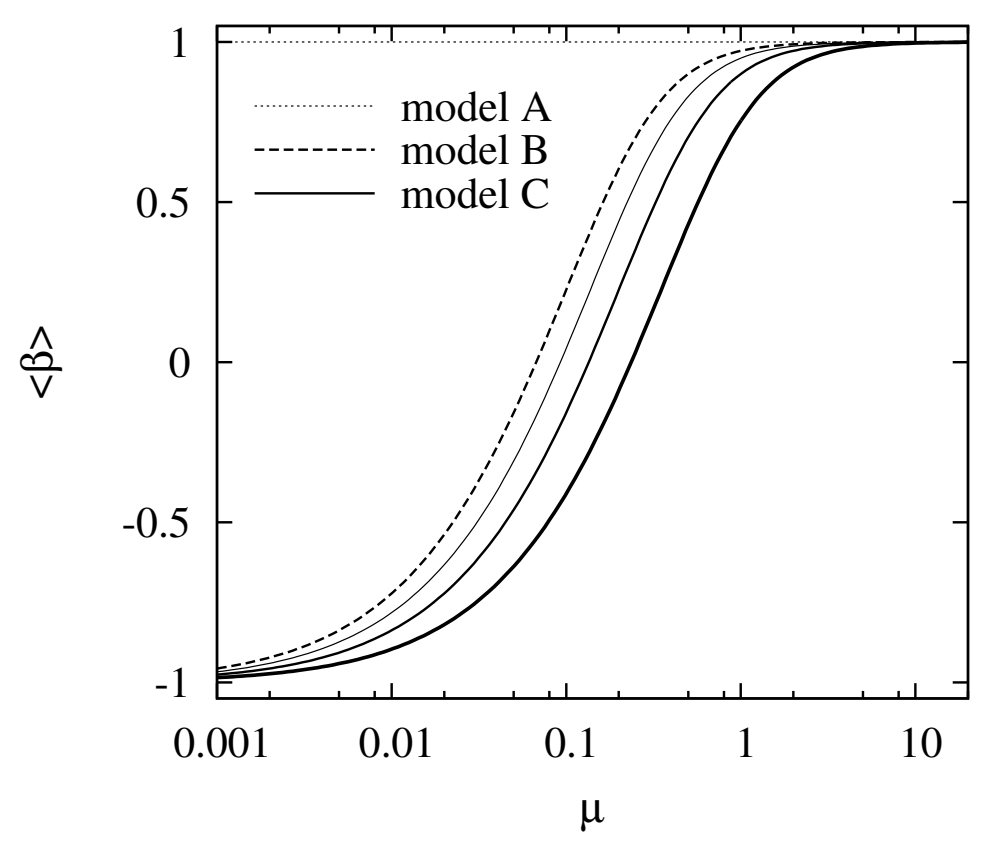

Figure 10.2.: Expected mean tangential restitution, $\langle\beta\rangle$, as function of the friction coefficient $\mu$ for models $\mathrm{A}, \mathrm{B}$, and $\mathrm{C}$. The parameters used are $\alpha=0.95, \beta_{0}=1.0$ (for $\mathrm{A}, \mathrm{B}$ ) and different $R=1.0$, 0.40 , and 0.15 (model C: solid lines from right to left).

\subsubsection{Model D: Simplified Coulomb Friction $\rightarrow \beta=\beta\left(\gamma_{12}\right)$}

In this section and in the following one, we discuss a coefficient of tangential restitution which depends on $\gamma$. Model $\mathrm{D}$ is defined by approximating $\gamma \approx \gamma_{12}$, which is strictly true for perfect head-on collisions or perfectly smooth particles only ( $\mu=0$ (or equivalently $\beta_{0}=-1$ ). We again obtain the same differential equations (10.4) for $T_{\text {rot }}$ and $T_{\text {tr }}$ with the coefficients

$$
\begin{aligned}
A=A_{\mu} & =A_{r}+\left[A_{\eta_{0}}+A^{*}\right] / f^{3} \\
B=B_{\mu} & =\left[B_{\eta_{0}}+B^{*}\right] / f \\
B^{\prime}=B_{\mu}^{\prime} & =\left[B_{\eta_{0}}+B^{\prime *}\right] / f^{3} \\
C & =C_{\mu}=\left[C_{\eta_{0}}+C^{*}\right] / f,
\end{aligned}
$$

and $f$ defined in Eq. (10.13). The correction terms due to Coulomb sliding are denoted by an asterisk. Defining

$$
\widetilde{B}^{*}:=-\frac{\eta_{0} c^{2}}{2 q}=-\frac{2 \eta_{0}^{3}}{q \mu^{2}(1+\alpha)^{2}} \leq 0
$$


which will be recovered as the correction term to $B$ in model E, cf. Eq. (10.23), they read

$$
\begin{aligned}
B^{*} & =-\frac{\eta_{0} \widetilde{B}^{*}}{(f+1)^{2}} \geq 0 \\
B^{*} & =-\frac{(2 f+1)}{(f+1)^{2}} \eta_{0} \widetilde{B}^{*}=(2 f+1) B^{*} \geq 0 \\
A^{*} & =q\left(\left|\widetilde{B}^{*}\right|-B^{\prime *}\right), \text { and } \\
C^{*} & =\frac{1}{2 q}\left(\eta_{1} f-\eta_{0}-2 B^{*}\right)
\end{aligned}
$$

expressed in terms of $\widetilde{B}^{*}$ [cf. Eq. (10.20)], $f$ [cf. Eq. (10.13)], $\eta_{0}$ [cf. Eq. (10.10)], $\eta_{1}$ [cf. Eq. (10.14)], and $q$ [cf. Eq. (2.7)]. The terms $B^{*}$ and $B^{\prime *}$ are strictly positive, while the dissipation correction terms $A^{*}$ and $C^{*}$, in principle, can change sign. Note also that $B^{*}$ and $B^{\prime *}$ are not identical here. All coefficients depend on the system parameters only. They are constants in time - in contrast to model $\mathrm{C}$ (and $\mathrm{E}$ as will be shown later).

\section{Constant tangential restitution limit}

In the limit $\mu \rightarrow \infty$, one has $c \rightarrow 0$, i.e. $E \rightarrow 0$, and $f \rightarrow 1$, and all correction terms $\left\{A^{*}, B^{*}, B^{\prime *}, C^{*}\right\} \rightarrow 0$ so that one obtains Eqs. (10.4)-(10.9). Note in particular that the coefficients $B_{\mu}$ and $B_{\mu}^{\prime}$ are equal only in the limit $\mu \rightarrow \infty$ [HZ97].

\section{Weak friction limit}

In the limit $\mu \rightarrow 0(c \rightarrow \infty, f \rightarrow c)$, the lowest order expansion in $c^{-1}$ leads to an approximation of the coefficients in Eqs. (10.19), where we have used $\eta_{0} / c=\mu(1+\alpha) / 2$ :

$$
\begin{aligned}
& B_{\mu}=\frac{\eta_{0}}{q} \frac{1+\alpha}{2} \mu+\mathcal{O}\left(\mu^{2}\right) \\
& B_{\mu}^{\prime}=\frac{1}{q}\left(\frac{1+\alpha}{2}\right)^{2} \mu^{2}+\mathcal{O}\left(\mu^{3}\right) \\
& A_{\mu}=A_{r}+\frac{1+\alpha}{4} \mu+\mathcal{O}\left(\mu^{2}\right) \\
& C_{\mu}=\frac{1}{2 q} \frac{1+\alpha}{2} \mu\left(|\ln (\mu)|+\ln \left(\frac{4 \eta_{0}}{1+\alpha}\right)-2 \frac{\eta_{0}}{q}\right)+\mathcal{O}\left(\mu^{2}\right) .
\end{aligned}
$$

From Eqs. (10.22), we learn that $B_{\mu}^{\prime}$ is second order in $\mu$, whereas $B_{\mu}$ is first order in $\mu$, reflecting an asymmetry in the energy transfer rates. On the other hand, $A_{\mu} \approx A_{r}$ is almost constant, whereas $C_{\mu}$ depends on $\mu$ logarithmically which is an artifact of our approximation $\gamma_{12} \sim \gamma$, see Eq. (10.25) below. 


\subsubsection{Model E: Real Coulomb Friction $\rightarrow \beta=\beta(\gamma)$}

Finally, we present the MF theory of the full Walton model. That means we use $\beta(\gamma)$, instead of $\beta\left(\gamma_{12}\right)$, to compute the coefficients. The calculation is similar to the one for 3D in Ref. [HHZ00] and is presented in appendix C. We obtain the following coefficients, to be inserted into Eqs. (10.4),

$$
\begin{aligned}
A & =\widetilde{A}_{\mu}(R)=A_{r}+\left[A_{\eta_{0}}+\widetilde{A}^{*}\right] / \tilde{f}^{3} \\
B & =\widetilde{B}_{\mu}(R)=\left[B_{\eta_{0}}+\widetilde{B}^{*}\right] / \tilde{f}^{3} \\
B^{\prime} & =\widetilde{B}_{\mu}^{\prime}(R)=\left[B_{\eta_{0}}+\widetilde{B}^{\prime *}\right] / \tilde{f}^{3} \\
C & =\widetilde{C}_{\mu}(R)=\left[C_{\eta_{0}}+\widetilde{C}^{*}\right] / \tilde{f}^{3},
\end{aligned}
$$

with the tilded asterisked correction terms

$$
\begin{aligned}
\widetilde{B}^{*} & =-\frac{\eta_{0} c^{2}}{2 q}=-\frac{2 \eta_{0}^{3}}{q \mu^{2}(1+\alpha)^{2}} \leq 0, \\
\widetilde{B}^{\prime *} & =-\frac{2 \tilde{f}+1}{(\tilde{f}+1)^{2}} \eta_{0} x^{4} \widetilde{B}^{*} \geq 0 \\
\widetilde{A}^{*} & =q\left(\left|\widetilde{B}^{*}\right|-\widetilde{B}^{\prime *}\right), \text { and } \\
\widetilde{C}^{*} & =-x^{2} \widetilde{B}^{*} \geq 0,
\end{aligned}
$$

where $\tilde{f} \equiv \tilde{f}(R)=\sqrt{1+x^{2} c^{2}}$ as introduced in Eq. (10.17). $x, c$, and $q$ have been defined in Eqs. (10.16), (2.9), and (2.7), respectively. Interestingly, we find now a negative $\widetilde{B}^{*}$ together with positive coefficients $\widetilde{B}^{\prime *}$ and $\widetilde{C}^{*}$. The coefficients $\widetilde{A}^{*}$ and $\widetilde{B}^{\prime *}$ are constructed in a way similar to $A^{*}$ and $B^{\prime *}$. In $\widetilde{B}^{\prime *}$ there appears an additional factor of $x^{4}$ and $f$ is replaced by $\widetilde{f}$. Like in model $\mathrm{C}$ but in contrast to models $\mathrm{A}, \mathrm{B}$, and $\mathrm{D}$, here the coefficients are again implicit functions of time.

In conclusion models $\mathrm{D}$ and $\mathrm{E}$ appear similar in shape but there are several striking differences: (i) The division by $f$ and $f^{3}$ in model $\mathrm{D}$ is in contrast with the division by $\tilde{f}^{3}$ in model $\mathrm{E}$, (ii) the term $\tilde{B}^{*}$ is always negative, while $B^{*}$ is positive, (iii) the term $\tilde{C}^{*}$ is always positive, while the sign of $C^{*}$ is not determined a-priori, (iv) among the correction terms of model $\mathrm{E}$, only $\tilde{B}^{*}$ is independent of $R$, and (v) the MF theory for the full Walton model appears in a simpler form, especially the term $\tilde{C}^{*}$.

\section{Constant tangential restitution limit}

The limit of constant tangential restitution can be reached by taking the limit $\mu \rightarrow \infty$. In this case $c \rightarrow 0, \tilde{f} \rightarrow 1$ and thus all additional coefficients $\widetilde{A}^{*}, \widetilde{B}^{*}, \widetilde{B}^{\prime *}$, and $\widetilde{C}^{*}$ vanish such that Eqs. (10.4)-(10.9) are recovered. 


\section{Weak friction limit}

In the limit $\mu \rightarrow 0(c \rightarrow \infty, \tilde{f} \rightarrow x c)$ an expansion to the lowest order in $\mu$ leads to an approximation of the coefficients in Eqs. (10.23) when we remember that $\eta_{0} / c=(1+\alpha) \mu / 2$ :

$$
\begin{aligned}
\widetilde{B}_{\mu}(R) & =-\frac{1}{2 q x^{3}} \frac{1+\alpha}{2} \mu+\mathcal{O}\left(\mu^{3}\right) \\
\widetilde{B}_{\mu}^{\prime}(R) & =\frac{1}{q}\left(\frac{1+\alpha}{2}\right)^{2} \mu^{2}+\mathcal{O}\left(\mu^{3}\right) \\
\widetilde{A}_{\mu}(R) & =A_{r}-q\left(\widetilde{B}_{\mu}(R)+\widetilde{B}_{\mu}^{\prime}(R)\right)+\mathcal{O}\left(\mu^{3}\right) \\
\widetilde{C}_{\mu}(R) & =-x^{2} \widetilde{B}_{\mu}(R)+\mathcal{O}\left(\mu^{3}\right) .
\end{aligned}
$$

Since $x=x(R)$ approaches one in the weak friction limit, both $\widetilde{B}_{\mu}(R)$ and $\widetilde{C}_{\mu}(R)$ are proportional to $\mu$ in leading order. To lowest order in $\mu$, Eq. (10.25) predicts $\widetilde{A}_{\mu}(R)=A_{r}+\mathcal{O}(\mu)$, i.e. proportional to $\mu^{0}$, while $\widetilde{B}_{\mu}^{\prime}(R)$ is proportional to $\mu^{2}$.

For $\mu \ll 1$, Eqs. (10.4) with (10.25) simplify to

$$
\frac{d}{d t} T_{\mathrm{tr}}(t)=H_{\mathrm{dr}}-G T_{\mathrm{tr}}^{3 / 2}\left(\frac{1-\alpha^{2}}{4}+\mathcal{O}(\mu)\right)
$$

which means that in the limit of low friction the differential equations for $T_{\operatorname{tr}}$ and $T_{\text {rot }}$ decouple. In the non-driven case this leads to surviving rotational energy (not shown, cf. appendix C.7), similar to Refs. [HHZ00, Gol04].

\subsection{Steady State}

Before discussing the approach to the stationary state in the next chapter, we first elucidate the stationary state and compare results of our simulations to various levels of refinements of the mean field theory.

\subsubsection{Analytical Results}

By imposing $\frac{d}{d t} T_{\mathrm{tr}}^{\mathrm{stat}}=0$ and $\frac{d}{d t} T_{\mathrm{rot}}^{\text {stat }}=0$ one gets the steady state values of the rotational and the translational temperatures as has already been discussed in some detail in chapter 3 . For models $\mathrm{A}, \mathrm{B}$ and $\mathrm{D}$, the coefficients in the differential equation do not depend on $R$ (or $x$ ). Therefore the solution is simply

$$
T_{\mathrm{rot}}^{\text {stat }}=R^{\mathrm{stat}} T_{\mathrm{tr}}^{\mathrm{stat}}, \text { and } T_{\mathrm{tr}}^{\mathrm{stat}}=\left(\frac{H_{\mathrm{dr}}}{G \mathcal{I}}\right)^{2 / 3}
$$


with

$$
R^{\text {stat }}=B^{\prime} / C \text {, and } \mathcal{I}=A-B R^{\text {stat }},
$$

as discussed in more detail for all models in the following.

\section{Model A}

For model A, the equilibrium steady state ratio of rotational to translational energies is

$$
R^{\mathrm{stat}}=\frac{q \eta_{0}}{q-\eta_{0}}
$$

and the energy dissipation factor is

$$
\mathcal{I}=\frac{1-\alpha^{2}}{4}-\frac{\eta_{0}}{2}\left(1-\eta_{0}\right)-\frac{\eta_{0}^{3}}{2\left(q-\eta_{0}\right)}
$$

Note here again that model A does not contain any dependence on the coefficient of friction $\mu$.

\section{Model B}

Model B evolves from model A, by just replacing $\eta_{0}$ by $\eta_{1}(\mu)=\left(\eta_{0} / c\right) \ln (c+f)$ from Eq. (10.14) in the above two Eqs. (10.29) and (10.30), so that, e.g.,

$$
R^{\mathrm{stat}}=\frac{q \eta_{1}}{q-\eta_{1}}=\frac{q\left(\eta_{0} / c\right) \ln (c+f)}{q-\left(\eta_{0} / c\right) \ln (c+f)}
$$

In the limit of small $\mu \ll 1$, the leading order terms are $R^{\text {stat }} \approx(1+\alpha) \mu|\ln \mu| / 2$ and $\mathcal{I} \approx \frac{1-\alpha^{2}}{4}+\mathcal{O}(\mu|\ln \mu|)$.

\section{Model D}

From model D, the following, more complex terms are obtained:

$$
R_{\mu}^{\text {stat }}=\frac{B_{\mu}^{\prime}}{C_{\mu}}=\frac{\left[B_{\eta_{0}}+B^{\prime *}\right]}{\left[C_{\eta_{0}}+C^{*}\right]} \frac{1}{f^{2}} \underset{\mu \ll 1}{\approx}(1+\alpha) \mu|\ln \mu|
$$

and

$$
\mathcal{I}_{\mu}=A_{\mu}-B_{\mu} R_{\mu}^{\text {stat }} \underset{\mu \ll 1}{\approx} \frac{1-\alpha^{2}}{4}+\mathcal{O}(\mu),
$$

so that, asymptotically for $\mu \ll 1$, model D leads to behavior similar to that of model B. 


\section{Model E}

Formally, we can write down Eqs. (10.27) for model E, too. Instead of using Eqs. (10.28), $R^{\text {stat }}$ must be extracted (numerically) from the second equation in Eqs. (10.4) with the coefficients from Eqs. (10.23) or, equivalently, from Eq. (C.58) in appendix C, Sec. C.2.2. The left hand side vanishes in the stationary case $\left(d T_{\text {rot }} / d t=0\right)$. As will be shown below there is always a unique solution for $R^{\text {stat }}$. With this solution for $R^{\text {stat }}$ at hand, the first equation in Eqs. (10.4) with the coefficients from Eqs. (10.23) or, equivalently, Eq. (C.31) in appendix C, Sec. C.2.1 can be written in the form $T_{\mathrm{tr}}^{\text {stat }}=\left(\frac{H_{\mathrm{dr}}}{G \mathcal{I}}\right)^{2 / 3}$ again. Here $\mathcal{I}$ is a nonlinear function of $R^{\text {stat }}$ whose particular form can be easily seen from Eq. (C.31) in appendix C, page 122.

To check if there is always a solution for $R \geq 0$ we check if there is always a solution $x \geq 1$ by considering Eq. (C.58), page 128. For $\mu \rightarrow \infty$ we see from Eq. (2.9) that $\cot \gamma_{0} \rightarrow 0$ and thus it is easy to see that Eq. (C.58) reduces to the quadratic equation yielding $x^{2}=$ $\left(1-\frac{1}{1+q} \frac{1+\beta_{0}}{2}\right)^{-1}>0$, i.e. there is exactly one root $x \geq 1$, i.e. $T_{\mathrm{rot}}^{\mathrm{stat}} / T_{\mathrm{tr}}^{\mathrm{stat}} \geq 0$. For $\cot ^{2} \gamma_{0}>$ 0 there is also exactly one solution $x \geq 1$ as will be shown in the following proof.

Proof: First we show that there is always at least one solution: We have to find the zeros of

$$
\begin{aligned}
f(x)=\left(\frac{\eta_{0}^{2} x^{2}}{2 q}-\right. & \left.\left(x^{2}-1\right) \frac{\eta_{0}}{2}\left(1+x^{2} \cot ^{2} \gamma_{0}\right)\right) \cot ^{2} \gamma_{0} \\
& +\frac{\eta_{0}^{2}}{q}\left(\left(1+x^{2} \cot ^{2} \gamma_{0}\right)^{3 / 2}-1-\frac{3}{2} x^{2} \cot ^{2} \gamma_{0}\right) .
\end{aligned}
$$

The last term in the big parentheses, which is proportional to $\frac{\eta_{0}^{2}}{q}$, is always greater then zero. So is the first part of the first term, $\frac{\eta_{0}^{2} x^{2}}{2 q}$. The remainder, which is proportional to $\left(x^{2}-1\right)$, is always negative. Now it is easy to see that for $x=1, f(x)>0$. For $x \rightarrow \infty, f(x) \rightarrow-\infty$ because the highest power of $x$ is $x^{4}$ with a negative sign. Since the function is continuous, there is always a solution for $x$ such that $f(x)=0$. [By the way: This is different for freely cooling systems where the rotational energy can survive while the translational energy decreases according to Haff's law [HHZ00], cf. appendix C.7.]

Now we show that the solution is unique. For this purpose we re-write Eq. (10.31) as

$$
\begin{array}{r}
f(x)=\frac{\eta_{0}}{2}\left(1+x^{2}\left[\cot ^{2} \gamma_{0}-\left(1-\frac{\eta_{0}}{q}\right)\right]-x^{4} \cot ^{2} \gamma_{0}\right) \cot ^{2} \gamma_{0} \\
+\frac{\eta_{0}^{2}}{q}\left(\left(1+x^{2} \cot ^{2} \gamma_{0}\right)^{3 / 2}-1-\frac{3}{2} x^{2} \cot ^{2} \gamma_{0}\right) .
\end{array}
$$


It can be easily seen that for $\cot ^{2} \gamma_{0} \geq\left(1-\frac{\eta_{0}}{q}\right)$ there is exactly one solution because in this case the only negative contribution to $f(x)$ is proportional to $x^{4}$. For $\cot ^{2} \gamma_{0}<\left(1-\frac{\eta_{0}}{q}\right)$ we show that the derivative $\frac{d}{d x} f(x)<0 \forall x$ by noting that

$$
\sqrt{1+x^{2} \cot ^{2} \gamma_{0}}<1+\frac{q}{3 \eta_{0}}\left(2 x^{2} \cot ^{2} \gamma_{0}+\left[1-\frac{\eta_{0}}{q}\right]-\cot ^{2} \gamma_{0}\right)
$$

for $\left[1-\frac{\eta_{0}}{q}\right]-\cot ^{2} \gamma_{0}>0$. Here we have used that $\frac{q}{\eta_{0}}>1$ for all possible parameters. Since the slope of $f(x)$ is negative for all $x>1$ there is only one zero.

\section{Models $\mathbf{C}$ and $\mathbf{E}$ for small $\mu$}

As already mentioned for model $\mathrm{E}$, the coefficients in the differential equations depend on $R$ for models $\mathrm{C}$ and $\mathrm{E}$, so that the steady state values have to be computed numerically for a general choice of parameters. Analytical results can only be achieved in the limit $\mu \ll 1$, where we can use the expansions of the coefficients introduced in Secs. 10.2.3 and 10.2.5.

For model $\mathrm{C}$ we obtain to lowest order in $\mu$, the dissipation factor $\mathcal{I} \approx A_{r}$ and, using $\eta_{2}=$ $\left(\eta_{0} / 2\right) \ln c$,

$$
R^{\mathrm{stat}} \underset{\mu \ll 1}{\approx} \frac{2 \eta_{2}}{c} \sqrt{1+\left(\frac{\eta_{2}}{c q}\right)^{2}}+\frac{2}{q}\left(\frac{\eta_{2}}{c}\right)^{2} \underset{\mu \ll 1}{\approx} \frac{1+\alpha}{2} \mu|\ln (\mu)|
$$

For model $\mathrm{E}$ we find again $\mathcal{I} \approx A_{r}$ and

$$
R^{\text {stat }} \underset{\mu \ll 1}{\approx} \frac{2 \eta_{0}}{c} \sqrt{1+\left(\frac{\eta_{0}}{c q}\right)^{2}}+\frac{2}{q}\left(\frac{\eta_{0}}{c}\right)^{2} \underset{\mu \ll 1}{\approx}(1+\alpha) \mu,
$$

very similar in shape to the result from model $\mathrm{C}$, besides the logarithm $\ln c$ that is hidden in the definition of $\eta_{2}$. This leads to the qualitative difference in asymptotic behavior between models $\mathrm{C}$ and $\mathrm{E}$ : The correct asymptotic behavior for small $\mu$ is $R^{\text {stat }} \propto \mu$. Note again that the more refined model $\mathrm{E}$ leads to a simpler analytical result than the approximated model $\mathrm{C}$.

\section{Discussion}

The expansions for small $\mu \ll 1$ show that the result for $R^{\text {eq }}$ based on model E, see Eq. (10.34), disagrees with all other models. In model $\mathrm{E}$ we find that $R^{\text {stat }}$ vanishes linearly as $\mu \rightarrow 0$, whereas models A-D predict a slower decrease, encoded in the $\mu|\ln \mu|$ dependence. Models A and B have the same analytical form for $R^{\text {stat }}$ if expressed in terms of $\eta_{0}$ for model A and in terms of $\eta_{1}$ for model B. Similarly, models $\mathrm{C}$ and $\mathrm{E}$ have the same functional dependence on $\eta$, 
if $\eta_{2}$ is used for model $\mathrm{C}$ and $\eta_{0}$ for model $\mathrm{E}$. The comparison of the models for arbitrary values of $\mu$ will be given in the next section, where we also present the results of our simulations and compare them to the predictions of the various mean field models.

\subsubsection{Comparison with Simulations}

In this section the steady state predictions from our models are confronted with the numerical simulation results. Note that we present results for rather high densities and dissipation, where our assumptions about homogeneity of the system and the Gaussian shape of the velocity distributions is not strictly true anymore. However, we want to stress the point that the present theory is astonishingly close to the numerical simulation with experimentally relevant parameters even when the most basic assumptions are somewhat questionable.

\section{Variation of $\beta_{0}$}
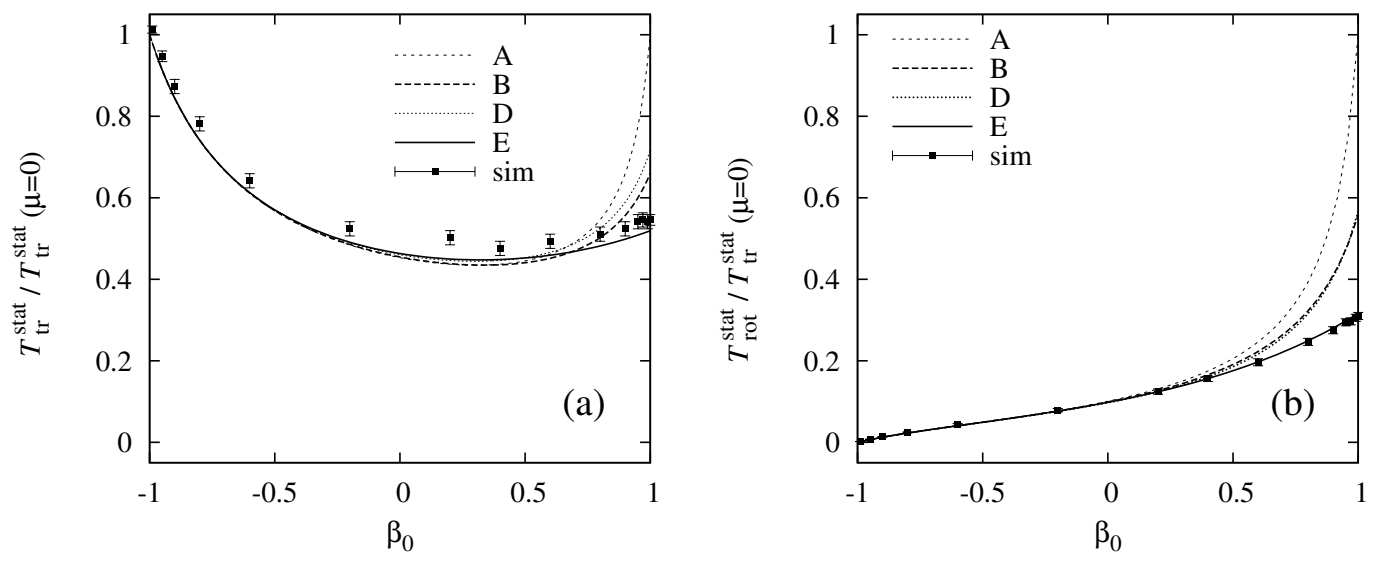

Figure 10.3.: Simulation results (symbols) and theory (lines) for the parameters $\phi=0.34, N=$ 11025, $\alpha=0.95$, and $\mu=0.5$, plotted against the maximum tangential restitution $\beta_{0}$ : (a) Translational temperature $T_{\mathrm{tr}}^{\mathrm{stat}}$, and (b) rotational temperature $T_{\mathrm{rot}}^{\mathrm{stat}}$, plotted against $\beta_{0}$, and scaled by $T_{\mathrm{tr}}^{\mathrm{stat}}(\mu=0)$, the mean field value for smooth particles.

In Figs. 10.3 (a) and (b) and Fig. 10.32 the equilibrium rotational and translational temperatures and their ratio $R$ are compared for $\alpha=0.95, \mu=0.5$ and different values of $\beta_{0}$. Note that the data in (a) and (b) are scaled with the expression for $\mu=0$. The symbols correspond to simulation data, with the error bars showing the standard deviation from the mean values. The lines correspond to different refinements of the theoretical approaches, i.e. models A, B, D, and E. 


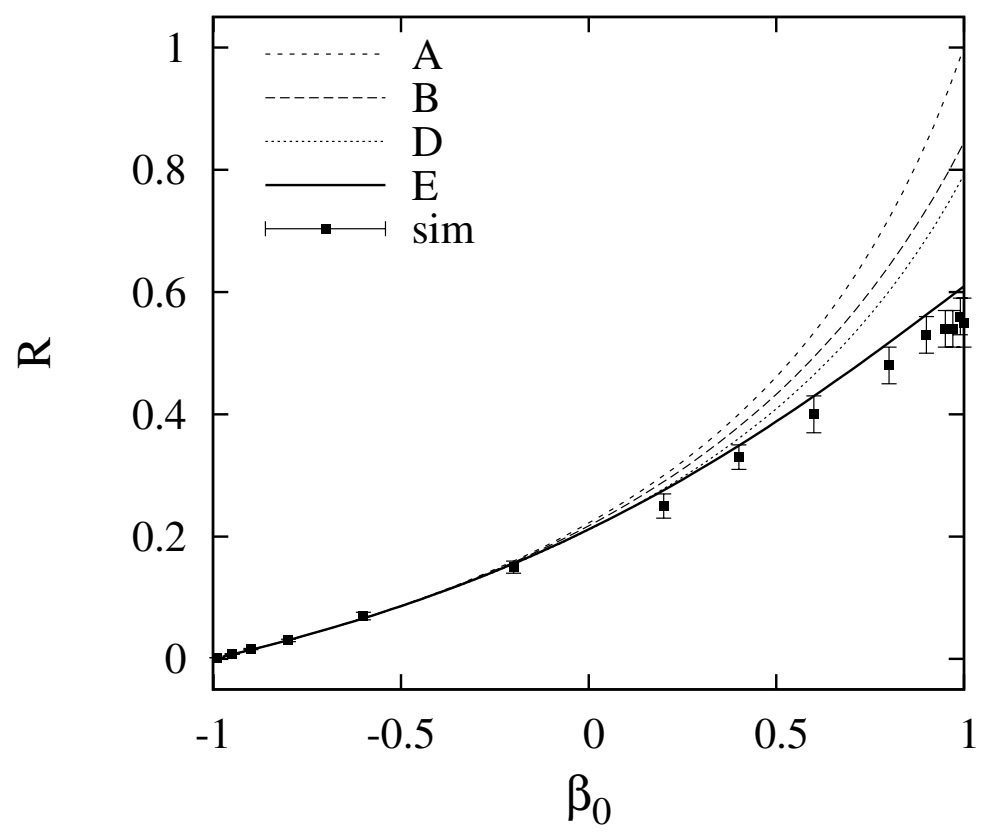

Figure 10.4.: Ratio of rotational and translational temperature $R$, plotted against $\beta_{0}$ for the same system as in Fig. 10.3.

For $\beta_{0} \approx-1$, the simulations agree with all theoretical predictions; for $\beta_{0} \approx 1$, large discrepancies are evident. The more refined a model used, the better the quality of agreement. The qualitative behavior of the data is best captured by model $\mathrm{E}$, and we relate the remaining quantitative deviations to the fact that the simulations involve rather high density $\phi$ and comparatively strong dissipation $\alpha$.

\section{Variation of $\mu$ - translational temperature}

In Fig. 10.5 we plot the translational temperature in the same way as in Fig. 10.3 (a), but now, we keep the values $\beta_{0}=0.4$ (a) and $\beta_{0}=1.0$ (b) fixed and vary $\mu$. Furthermore, we compare data for $\alpha=0.99$ and $\alpha=0.95$ in one plot and observe satisfactory agreement between simulation results and the full mean field theory, model E. (The predictions from models A and B are only shown for $\alpha=0.99$.)

For (realistic) values of $\beta_{0}=0.4$, see Fig. 10.5 (a), one obtains a transition from the $\mu=0$ limit to the $\mu \rightarrow \infty$ value of the kinetic energy, over two orders of magnitude in $\mu$, whereas for $\beta_{0}=1.0$, see Fig. 10.5 (b), the kinetic energy first decays with $\mu$ but then increases again to the equilibrium temperature of smooth particles, since no energy is dissipated due to tangential friction for $\mu \rightarrow \infty$ and $\beta_{0}=1.0$. 

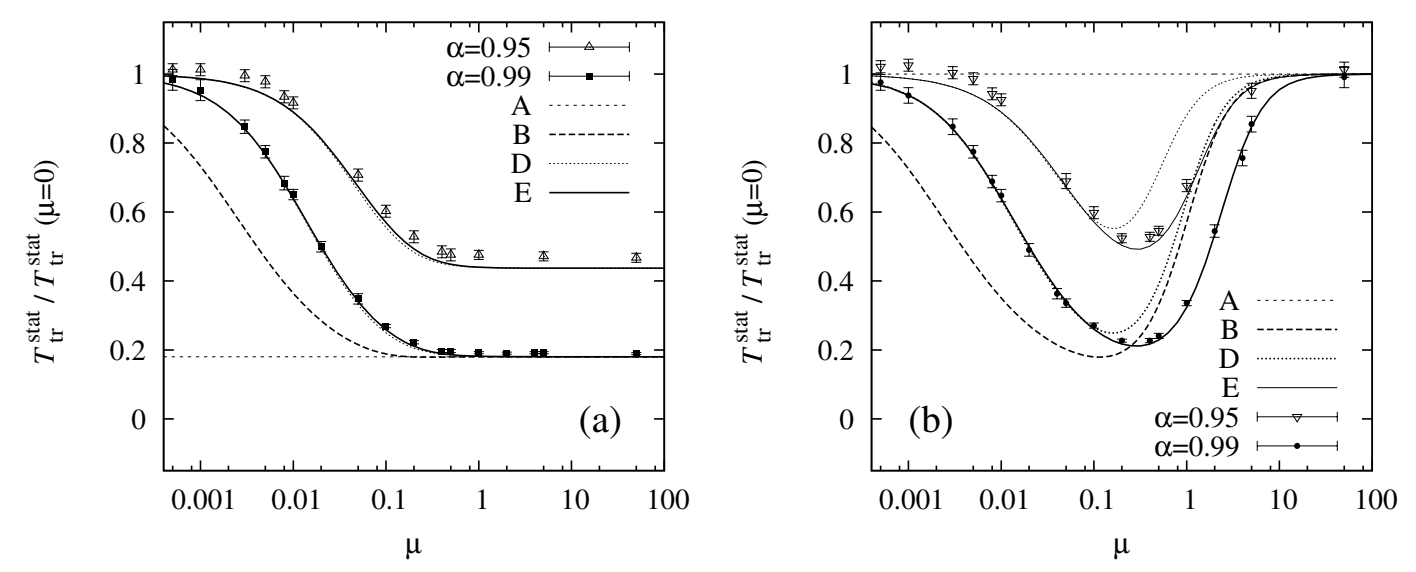

Figure 10.5.: Translational temperature $T_{\mathrm{tr}}^{\text {stat }}$ scaled by the mean field value for smooth particles $T_{\mathrm{tr}}^{\text {stat }}(\mu=0)$, plotted against $\mu$, for the parameters as in Fig. 10.3. The tangential restitution coefficients are fixed to (a) $\beta_{0}=0.4$, and (b) $\beta_{0}=1.0$. Data with normal restitution $\alpha=0.99$ (solid symbols and thick lines) and $\alpha=0.95$ (open symbols and thin lines) are compared.

We remark that model $\mathrm{A}$, with $\beta=\beta_{0}$ and the limit $\mu \rightarrow \infty$ is inadequate to model the $\mu$-dependency of the data, as expected. It is correct only in the limit $\mu \rightarrow \infty$. Approach B only shows qualitative agreement with our simulation data, whereas theory D shows good quantitative agreement for small $\mu$. The agreement seems better for weak normal dissipation $\alpha=0.99$, as compared to the cases with $\alpha=0.95$. The deviations between simulations and model $\mathrm{D}$ in the intermediate range of $\mu$ are due to values of $R$ of the order of unity, for which the assumption $\gamma_{12} \approx \gamma$ is not true, as pointed out above.

For weaker normal dissipation $r$, one obtains a stronger reduction of the translational temperature in the range of strongest total dissipation (around $\mu \approx 0.4$ ). This is due to the comparatively stronger contribution of tangential dissipation. However, as in the previous section, the agreement between simulations and model $\mathrm{E}$ is satisfactory.

\section{Variation of $\mu$ - rotational temperature}

In Fig. 10.6 we plot the ratio of rotational and translational temperature in the same way as in Fig. 10.4, but now, like in Fig. 10.5, we keep the values $\beta_{0}=0.4$ (a) and $\beta_{0}=1.0$ (b) fixed and vary $\mu$. We also compare data for $\alpha=0.99$ and $\alpha=0.95$ in one plot. For the values of $\beta_{0}$ examined (see Fig. 10.6) one observes a smooth transition of $R$ over about two orders of magnitude in $\mu$, from the value $R=0$ (in the limit $\mu=0$ ) to the value $R=\beta_{0}$ (in the limit $\mu \rightarrow \infty)$. Note that the observation $R=\beta_{0}$ is coincidence, since the correct asymptotic result for large $\mu$ is $R=2\left(1+\beta_{0}\right) /\left(9-5 \beta_{0}\right)$. Again, the agreement between simulations and model $\mathrm{E}$ is excellent. 

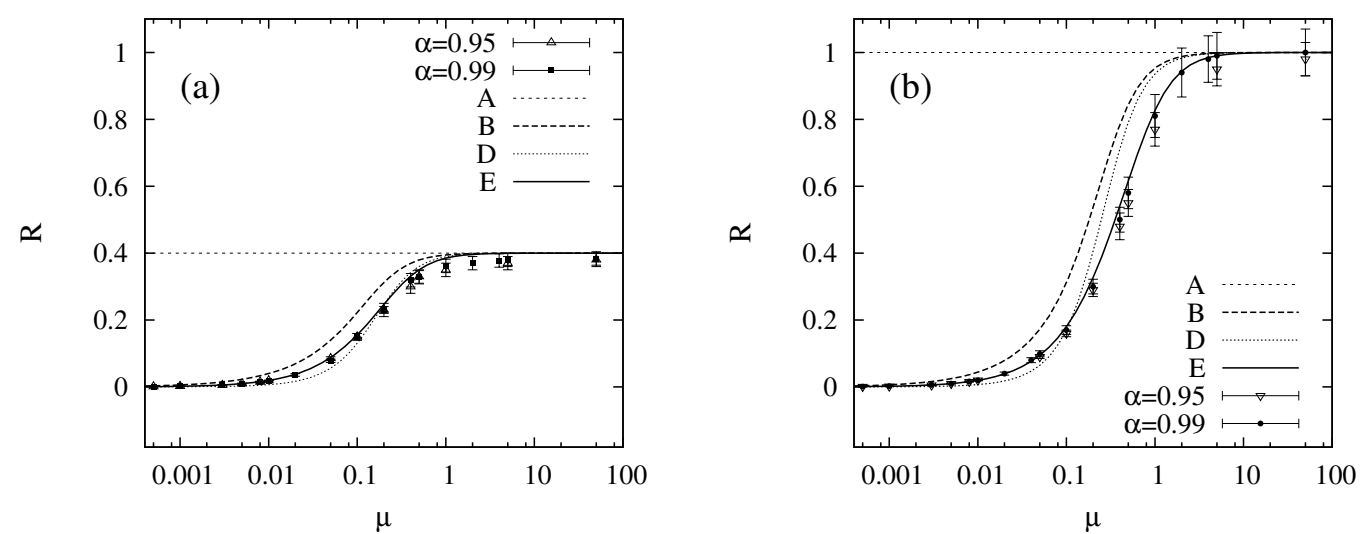

Figure 10.6.: $R$ plotted against $\mu$, for the same parameters as in Fig. 10.5. The tangential restitution coefficients are again fixed to (a) $\beta_{0}=0.4$, and (b) $\beta_{0}=1.0$.
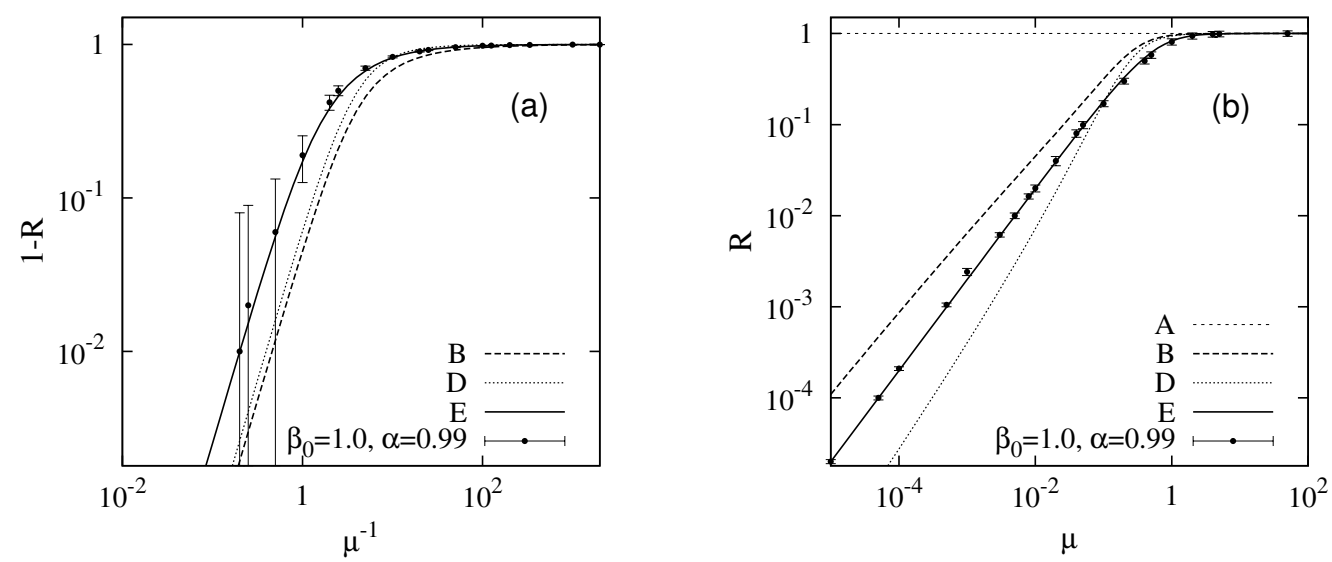

Figure 10.7.: (a) Deviation from equipartition, $1-R$, plotted against the inverse friction coefficient, $\mu^{-1}$, and (b) ratio of rotational and translational temperature, $R$, plotted against $\mu$, both for the same simulations as in Fig. 10.5 (b). Note the double-logarithmic scale of these plots.

All models agree qualitatively in the large $\mu$-limit, even though the quantitative agreement with simulations is again best caught by model E, as can be seen in Fig. 10.7 (a).

The remaining question is the asymptotic behavior for very small $\mu$, as can be viewed in Fig. 10.7 (b), and as discussed theoretically in Sec. 10.3.1. The quantitative behavior of $R$ for small $\mu$ is tested by a power law fit of the numerical values, according to an expression $R=b \mu^{r}$. The fit gives $r=1.00(4)$, for $\alpha=0.99, \beta_{0}=0.4$ or 1.0 and $r=0.99(4)$, for $\alpha=0.95$, $\beta_{0}=0.4$ or 1.0. Thus the asymptotic behavior is proportional to $\mu$, in excellent qualitative and quantitative agreement with the prediction of model $\mathrm{E}$. 


\subsection{Approach to the Steady State}

\subsubsection{Close to the Steady State}

Provided the system is sufficiently close to steady state, we can linearize the set of Eqs. (10.4) around $T_{\mathrm{tr}}^{\mathrm{stat}}$ and $T_{\mathrm{rot}}^{\text {stat }}$. This is particularly simple for models A, B, and D, where the coefficients in the differential equation do not depend on $R$ and hence can be solved analytically for the stationary state. We set $T_{\mathrm{tr}}(t)=T_{\mathrm{tr}}^{\mathrm{stat}}\left(1+\delta T_{\mathrm{tr}}(t)\right)$ and $T_{\text {rot }}(t)=T_{\text {rot }}^{\text {stat }}\left(1+\delta T_{\text {rot }}(t)\right)$ and obtain the linearized dynamic equations

$$
\begin{aligned}
\frac{d}{d t} \delta T_{\mathrm{tr}} & =G T_{\mathrm{tr}}^{\mathrm{stat}}\left\{\left(\frac{3}{2} A+\frac{B B^{\prime}}{2 C}\right) \delta T_{\mathrm{tr}}+\frac{B B^{\prime}}{C} \delta T_{\text {rot }}\right\} \\
\frac{d}{d t} \delta T_{\text {rot }} & =2 G C T_{\mathrm{tr}}^{\text {stat }}\left\{\delta T_{\mathrm{tr}}-\delta T_{\text {rot }}\right\} .
\end{aligned}
$$

This set of linear equations is easily solved to yield two relaxation rates $\lambda_{1}$ and $\lambda_{2}$. In a stable stationary state they must be positive and they are. Here we present only results for the simplest model (A) and postpone the general discussion to the next paragraph, where the full dynamic evolution towards steady state will be examined.

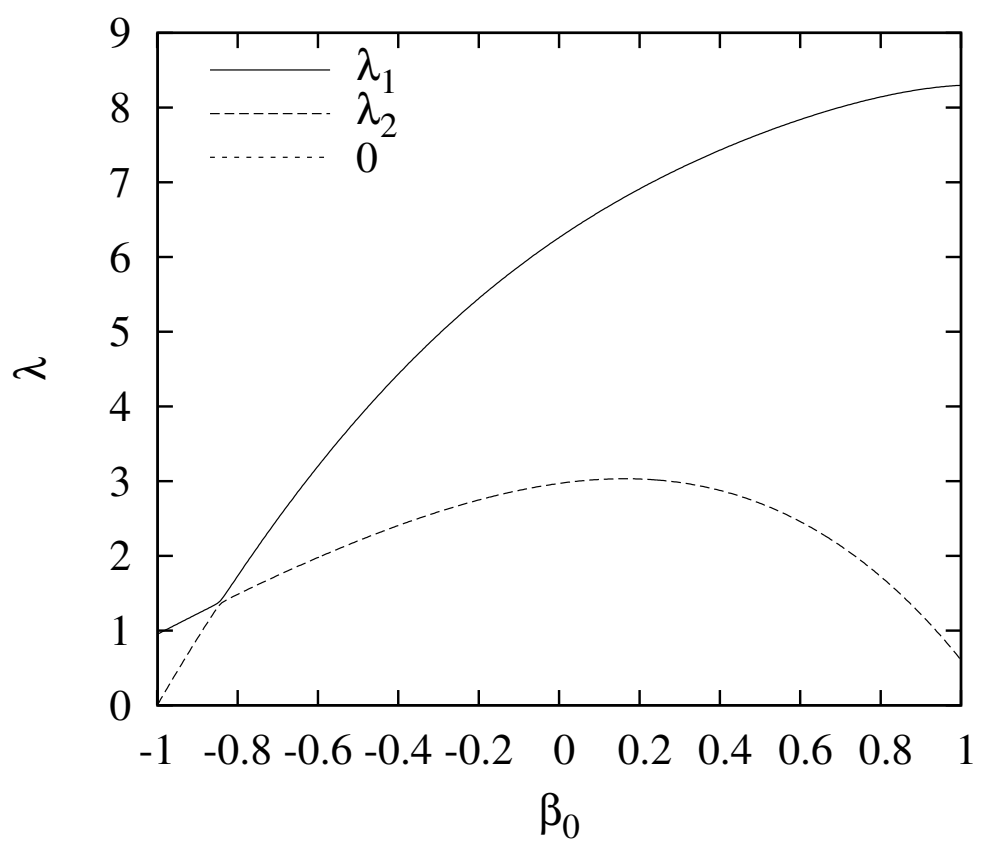

Figure 10.8.: Relaxation rates $\lambda_{1,2}$, close to steady state for $\alpha=0.95$ as a function of $\beta_{0}$.

In Fig. 10.8, we plot the two relaxation rates as a function of $\beta_{0}$ for a fixed value of $\alpha=0.95$. In the limit of smooth spheres one of the rates vanishes because the rotational energy is conserved in that limit. For $\beta_{0} \sim-0.84$ the two rates are equal and for increasing $\beta_{0}$ the difference 
between the two rates increases monotonically with $\beta_{0}$, such that for perfectly rough spheres the larger rate is about fourteen times the smaller one. Such a pronounced separation of time scales is familiar from the cooling dynamics of the same model, see Ref. [HHZ00]. There it was shown that the ratio of translational to rotational energy, $R$, relaxes fast to its stationary value, whereas both the translational as well as the rotational energy decay on the same, much longer time scale. This point will be discussed in a more general setting (model $\mathrm{E}$ and relaxation from an arbitrary initial condition) in the subsequent paragraph.

\subsubsection{Full Dynamic Evolution}

In Fig. 10.9, the full dynamic evolution of the translational and rotational temperatures with time is shown for two simulations with $N=11025, \phi=0.0866, \alpha=0.95, \beta_{0}=1.0$, and different values for the coefficient of friction. In both situations the agreement between simulations and the numerical solution for the MF theory of the full Walton model, model $\mathrm{E}$, is good - not only concerning the limiting values and the asymptotes, but also the time dependence during the two regimes (i) equilibration between $T_{\operatorname{tr}}$ and $T_{\text {rot }}$, and (ii) approach to final steady state.
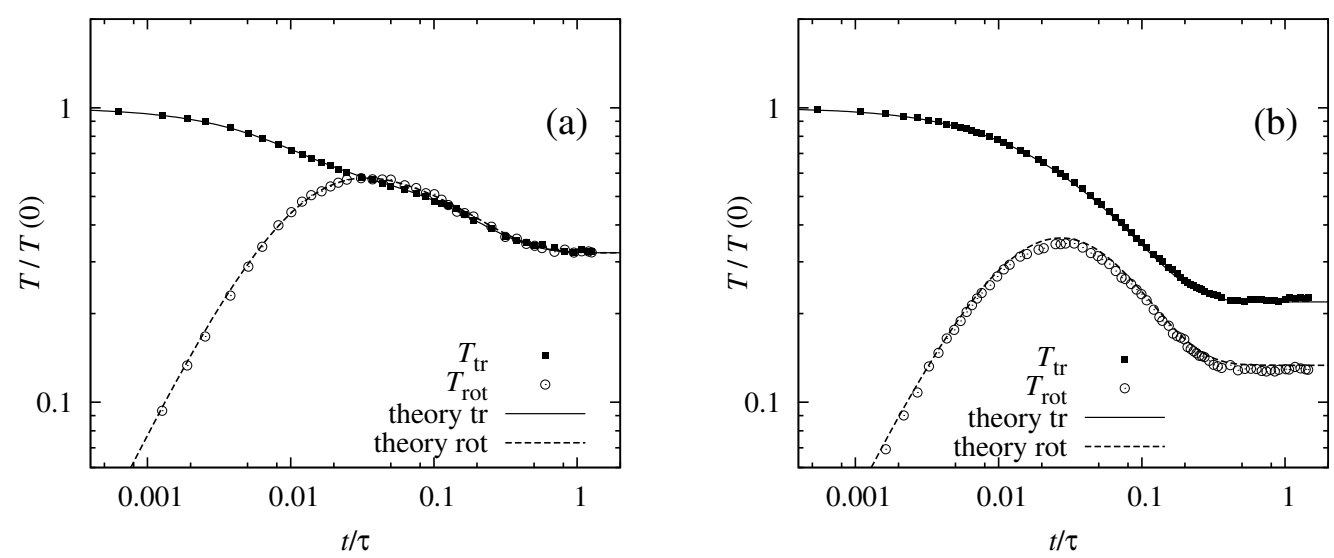

Figure 10.9.: Evolution of temperatures with rescaled time, with $\tau^{-1}=(1 / 2) G T_{\operatorname{tr}}(0)^{1 / 2}$, for simulations with $N=11025, \phi=0.0866, \alpha=0.95, \beta_{0}=1.0$, and (a) $\mu=\infty$, (b) $\mu=0.5$.

\subsection{Discussion}

A dynamic MF theory for the full time evolution of the translational and rotational temperatures of a homogeneously driven two-dimensional granular gas has been presented. Particle collisions were modeled using the Walton model [WB86], i.e. with normal dissipation, tangential restitution (sticking) and Coulomb friction (sliding). The Walton model can be formulated 
in terms of a coefficient of tangential restitution, which depends on the impact angle $\gamma$. Using a Pseudo-Liouville operator we have computed the distribution of impact angles as well as the mean field dynamics and steady state values of the translational and rotational temperatures.

In addition to the mean field theory of the Walton model ("model E"), we discussed three levels of approximation in order to simplify the differential equations of the time evolution. The crudest approximations including Coulomb friction ("model B" and "model C") assume that an effective constant coefficient of tangential restitution exists and can be computed by averaging over the angular distribution of impact angles. For model $\mathrm{C}$ this averaged coefficient depends on the current values of the translational and rotational temperatures and thus on time. Even simpler is model B where the rotational contribution to the impact angle is neglected, leading to a coefficient of tangential restitution that only depends on global system parameters. The closest approximation ("model D") to the full mean field theory ("model E") keeps the dependence of $\beta(\gamma)$ on the impact angle $\gamma$ but, like for model $\mathrm{B}$, the contribution of the rotation of the particles to the impact angle is neglected.

The predictions of the increasingly refined models of frictional dissipation as well as the full MF theory have been compared to simulations of a randomly driven mono-layer of spheres using an Event Driven algorithm. Emphasis has been put on the stationary state which is characterized by two temperatures, $T_{\mathrm{tr}}$ and $T_{\text {rot }}$, one for the translational and one for the rotational degrees of freedom. Guided by the MF approach we discovered a rich phenomenology like a non-trivial dependence of the stationary state temperatures on the model parameters. For example, the translational temperature is non-monotonic as a function of maximal tangential restitution $\beta_{0}$ and also non-monotonic as a function of Coulomb friction $\mu$, provided $\beta_{0}$ is sufficiently large.

All models predict steady state values of the translational and rotational temperatures, which are considerably improved as compared to the model without friction ("model A"), which assumes constant coefficients tangential restitution (see Figs. 10.5 and 10.6). All approximations A-E agree in the limit of large friction, where the tangential restitution becomes independent of the impact angle (see Fig. 2.2). Qualitative agreement between models B-D and simulations is achieved also for intermediate values of $\mu$. However in the limit $\mu \rightarrow 0$ all approximations break down and only the complete mean field solution ("model E") is in agreement with the simulations (see Fig. 10.7 (b)). In particular model E predicts the linear dependence of the ratio of temperatures, $R=T_{\text {rot }} / T_{\mathrm{tr}}$, on the friction coefficient $\mu$ that is observed in the simulations and was used in Ref. [JZ02] to derive an approximate kinetic theory of frictional particles.

Sticking contacts become more important relative to sliding contacts for fixed $\mu$ and decreasing $\beta_{0}$. In this regime models $\mathrm{B}$ and $\mathrm{D}$ seem reasonable, but lead to poor quantitative agreement as $\beta_{0}$ approaches 1 . The mean field theory of the full Walton model ("model E") leads to reasonable agreement for all values of $\beta_{0}$. For weak dissipation, $\alpha \rightarrow 1$, the agreement is very 
good - for stronger dissipation, we relate the deviations to the failure of both the homogeneity assumption and the molecular chaos assumption.

The mean field theory of the full Walton model ("model E") as well as model C lead to two highly non-linear coupled differential equations for the translational and rotational temperatures. In contrast, models B and D reduce to the same simple differential equations (10.4) that have been found for the dynamic behavior of rough particles with a constant coefficient of tangential restitution ("model A"). The only difference is that the constant coefficients $A, B, B^{\prime}$, and $C$ for model A that must be used in the simple differential equations (10.4) become more complicated functions of the global system parameters for models B and D. For very high friction $(\mu \rightarrow \infty)$ sliding becomes a negligible effect compared to sticking and all approximations as well as the mean field theory of the full Walton model simplify to the model with a constant coefficient of tangential restitution ("model A").

Linearizing the dynamic MF equations around the steady state leads to an eigenvalue problem with two relaxation rates, one of them being related to the equilibration between the translational and the rotational degrees of freedom, while the other one controls the approach of the system to its steady state. For strong coupling, the former process is much faster, so that there is a clear separation of time scales, which has been discussed already for a freely cooling system in the absence of driving.

In conclusion, realistic Coulomb friction turned out to be a subtle problem as only the mean field theory of the full Walton model predicts the effects of friction for all values of $\mu$ and $\beta_{0}$. All simplifications are both qualitatively and quantitatively wrong in some parameter range. 


\section{Summary and Outlook}

In this thesis we have explored two-dimensional driven granular gases in Event-Driven simulations. Our main focus was on the steady-state properties of a granular gas of smooth particles driven by vibrating walls. These systems depend not only on the global density but also on the distance between the driving walls as this distance must stay finite in order for boundary induced driving to be effective. We have measured the hydrodynamic fields for a wide range of parameters for these non-equilibrium stationary systems. In particlular, the full stress tensor and the full heat flux, both including collisional contributions have been obtained. These collisional contributions are often ignored and have not been measured before in vibrated systems.

In a quest for a local equation of state we have related the local pressure to the local temperature and density. This allowed us to obtain a constitutive relation for the pressure directly from the simulations and test its dependency on the system parameters. It has never been verified before whether a local equation of state indeed exists for driven granular gases, even though various proposals have been used. For small inelasticities the constitutive relation extracted from the simulations is largely independent of the global system parameters and can therefore be regarded as a local equation of state. In systems of higher inelasticity, the constitutive relation does depends on the global area fraction as well as on the geometry of the sample. This makes the hydrodynamic description severely more complex, even though it does not question it in general.

The generalization of Fourier's law of thermal conductance for granular systems relates the local heat flux to local gradients of density and temperature. Again, its validity has never been tested. To do so, we fitted the measured heat flux to the generalized Fourier law and extracted the corresponding transport coefficients which we assumed to be constant throughout the sample. As long as the temperature and density inhomogeneities stay sufficiently low and the systems are not too inelastic, the fit to the measured data is very good. In particular, the two-parameter fit is still very good at moderate inelasticities where the standard Fourier law fails. For larger gradients and/or stronger inelasticities the fit becomes worse and deviations can no longer be ignored. Plotting the corresponding transport coefficients as a function of the coefficient of restitution exhibits a strikingly non-monotonic behavior. The details of this non-monotonicity depend on the global area fraction.

Furthermore, we have measured local velocity distributions. In particular, the method used allowed us to obtain the very-high-velocity tails in much greater detail than usually found in the 
literature. These tails are different for the two velocity components parallel and perpendicular to the driving walls. Moreover, they depend on the position in the sample as well as on the coefficient of restitution and the global area fraction. Interestingly, even in the middle of the sample a signature of the driving walls can be found in the high velocity tails.

Finally we explored homogeneously driven systems of rough particles with Coulomb friction as described by the Walton model. Using a Pseudo-Liouville operator we calculated the dynamic evolution of the translational and rotational granular temperatures in mean field theory and compared the results to Event-Driven simulations. The agreement is very good even though we assumed Gaussian velocity distributions, molecular chaos, and spatial homogeneity in the analytic calculations. Additionally, we have introduced effective coefficients of tangential restitution in three levels of approximation to the full Walton model. Using these coefficients to calculate the mean field granular temperatures we find that the crudest approximation already captures the behavior of the simulations qualitatively, while only the full Walton model is able to predict the correct behavior for the full range of parameters.

To conclude, one has to acknowledge that the stationary state of a driven granular gas is by no means universal. In contrast to the corresponding elastic systems it shows peculiar features that depend on the details of the driving mechanism as well as on the geometry of the system, the boundary conditions and the precise values of the system parameters.

We plan to extend our studies in various directions. As a further step towards full granular hydrodynamics of realistic particles it is straightforward to also investigate hydrodynamic fields and velocity distributions of rough particles with Coulomb friction between vibrating walls. Again, the question arises to what extent the additional parameters affect the local equation of state, generalization of Fourier's law, and velocity distributions. In this context it would be particularly interesting to study the effects of gravity for better comparison with experiments and also to investigate three-dimensional systems. Polydisperse mixtures of particles with different material constants and different sizes could be studied as well. Finally, one might also investigate other driving mechanisms, like vibrations with finite frequency and non-zero amplitude. Other possibilities are sinusoidal driving, walls at fixed temperatures, and sheared systems. Work along these lines is in progress. 


\section{A. Heat Flux for Non-Zero Velocity Fields}

Here we recall the derivation of the general case where the velocity field $\boldsymbol{V}(\boldsymbol{r}, t)$ is non-zero. In this case, arriving at an equation similar to (4.35) is a little more elaborate but straightforward. The result will be Eqs. (4.36) and (4.37). When it cannot be assumed that the velocity field is zero the term within the parentheses at the right hand side of Eq. (4.28) on page 26 reads

$$
\begin{aligned}
& \sum_{i=1}^{N}\left(\frac{m_{i}}{2}|\boldsymbol{V}(\boldsymbol{r}, t)|^{2}\left(\boldsymbol{V}(\boldsymbol{r}, t)+\tilde{\boldsymbol{v}}_{\boldsymbol{i}}(\boldsymbol{r}, t)\right)+\frac{m_{i}}{2}\left|\tilde{\boldsymbol{v}}_{\boldsymbol{i}}(\boldsymbol{r}, t)\right|^{2}\left(\boldsymbol{V}(\boldsymbol{r}, t)+\tilde{\boldsymbol{v}}_{\boldsymbol{i}}(\boldsymbol{r}, t)\right)\right. \\
& \left.\quad+m_{i} \tilde{\boldsymbol{v}}_{\boldsymbol{i}}(\boldsymbol{r}, t) \cdot \boldsymbol{V}(\boldsymbol{r}, t)\left(\boldsymbol{V}(\boldsymbol{r}, t)+\tilde{\boldsymbol{v}}_{\boldsymbol{i}}(\boldsymbol{r}, t)\right)\right) \Phi\left(\boldsymbol{r}-\boldsymbol{r}_{\boldsymbol{i}}\right)
\end{aligned}
$$

Noting that $\sum_{i=1}^{N} m_{i} \tilde{\boldsymbol{v}}_{\boldsymbol{i}}(\boldsymbol{r}, t) \Phi\left(\boldsymbol{r}-\boldsymbol{r}_{\boldsymbol{i}}\right)=\boldsymbol{p}(\boldsymbol{r}, t)-\rho_{m}(\boldsymbol{r}, t) \boldsymbol{V}(\boldsymbol{r}, t)=0$, this simplifies to

$$
\begin{aligned}
& \sum_{i=1}^{N}\left(\frac{m_{i}}{2}|\boldsymbol{V}(\boldsymbol{r}, t)|^{2} \boldsymbol{V}(\boldsymbol{r}, t)+\frac{m_{i}}{2}\left|\tilde{\boldsymbol{v}}_{\boldsymbol{i}}(\boldsymbol{r}, t)\right|^{2} \tilde{\boldsymbol{v}}_{\boldsymbol{i}}(\boldsymbol{r}, t)\right. \\
& \left.\quad+\frac{m_{i}}{2}\left|\tilde{\boldsymbol{v}}_{\boldsymbol{i}}(\boldsymbol{r}, t)\right|^{2} \boldsymbol{V}(\boldsymbol{r}, t)+m_{i}\left[\tilde{\boldsymbol{v}}_{\boldsymbol{i}}(\boldsymbol{r}, t) \cdot \boldsymbol{V}(\boldsymbol{r}, t)\right] \tilde{\boldsymbol{v}}_{\boldsymbol{i}}(\boldsymbol{r}, t)\right) \Phi\left(\boldsymbol{r}-\boldsymbol{r}_{\boldsymbol{i}}\right)
\end{aligned}
$$

As already defined in Eq, (4.29), the kinetic part of the heat flux is

$$
\boldsymbol{q}^{\mathrm{kin}}(\boldsymbol{r}, t):=\sum_{i=1}^{N} \frac{m_{i}}{2}\left|\tilde{\boldsymbol{v}}_{\boldsymbol{i}}(\boldsymbol{r}, t)\right|^{2} \tilde{\boldsymbol{v}}_{\boldsymbol{i}}(\boldsymbol{r}, t) \Phi\left(\boldsymbol{r}-\boldsymbol{r}_{\boldsymbol{i}}\right)
$$

Noting that $\sum_{i=1}^{N} \frac{m_{i}}{2}\left|\tilde{\boldsymbol{v}}_{\boldsymbol{i}}(\boldsymbol{r}, t)\right|^{2} \boldsymbol{V}(\boldsymbol{r}, t) \Phi\left(\boldsymbol{r}-\boldsymbol{r}_{\boldsymbol{i}}\right)=\rho(\boldsymbol{r}, t) T(\boldsymbol{r}, t) \quad \boldsymbol{V}(\boldsymbol{r}, t)$ and $\sum_{i=1}^{N} m_{i}\left[\tilde{\boldsymbol{v}}_{\boldsymbol{i}}(\boldsymbol{r}, t) \cdot \boldsymbol{V}(\boldsymbol{r}, t)\right] \tilde{\boldsymbol{v}}_{\boldsymbol{i}}(\boldsymbol{r}, t) \Phi\left(\boldsymbol{r}-\boldsymbol{r}_{\boldsymbol{i}}\right)=\sigma_{k l}^{\mathrm{kin}}(\boldsymbol{r}, t) V_{l}(\boldsymbol{r}, t) \boldsymbol{e}_{\boldsymbol{k}}$ (sum over double indices) we can write

$$
\begin{aligned}
\frac{\partial}{\partial t} \mathcal{E}(\boldsymbol{r}, t)=\frac{1}{\Delta t} \sum_{i=1}^{N}\left(\Delta E_{i}\right) \Phi\left(\boldsymbol{r}-\boldsymbol{r}_{\boldsymbol{i}}\right)-\nabla_{\boldsymbol{r}} \cdot( & \frac{m}{2} \rho(\boldsymbol{r}, t)|\boldsymbol{V}(\boldsymbol{r}, t)|^{2} \boldsymbol{V}(\boldsymbol{r}, t) \\
& +\boldsymbol{q}^{\mathrm{kin}}(\boldsymbol{r}, t)+\rho(\boldsymbol{r}, t) T(\boldsymbol{r}, t) \boldsymbol{V}(\boldsymbol{r}, t) \\
& \left.+\sigma_{k l}^{\mathrm{kin}}(\boldsymbol{r}, t) V_{l}(\boldsymbol{r}, t) \boldsymbol{e}_{\boldsymbol{k}}\right)
\end{aligned}
$$


Using $\mathcal{E}(\boldsymbol{r}, t)=\frac{m}{2} \rho(\boldsymbol{r}, t)|\boldsymbol{V}(\boldsymbol{r}, t)|^{2}+\frac{2}{d} \rho(\boldsymbol{r}, t) T(\boldsymbol{r}, t)$, where $d$ is the dimension we get

$$
\begin{aligned}
\frac{\partial}{\partial t}\left[\frac{2}{d} \rho(\boldsymbol{r}, t) T(\boldsymbol{r}, t)\right]+\frac{\partial}{\partial t}\left[\frac{m}{2} \rho(\boldsymbol{r}, t)|\boldsymbol{V}(\boldsymbol{r}, t)|^{2}\right]= & \frac{1}{\Delta t} \sum_{i=1}^{N}\left(\Delta E_{i}\right) \Phi\left(\boldsymbol{r}-\boldsymbol{r}_{\boldsymbol{i}}\right) \\
& -\boldsymbol{V}(\boldsymbol{r}, t) \cdot \nabla_{\boldsymbol{r}}\left(\frac{m}{2} \rho(\boldsymbol{r}, t)|\boldsymbol{V}(\boldsymbol{r}, t)|^{2}\right) \\
& -\frac{m}{2} \rho(\boldsymbol{r}, t)|\boldsymbol{V}(\boldsymbol{r}, t)|^{2} \nabla_{\boldsymbol{r}} \cdot \boldsymbol{V}(\boldsymbol{r}, t) \\
& -\nabla_{\boldsymbol{r}} \cdot \boldsymbol{q}^{\mathrm{kin}}(\boldsymbol{r}, t) \\
& -\boldsymbol{V}(\boldsymbol{r}, t) \cdot \nabla_{\boldsymbol{r}}[\rho(\boldsymbol{r}, t) T(\boldsymbol{r}, t)] \\
& -\rho(\boldsymbol{r}, t) T(\boldsymbol{r}, t) \nabla_{\boldsymbol{r}} \cdot \boldsymbol{V}(\boldsymbol{r}, t) \\
& -\nabla_{\boldsymbol{r}} \cdot\left(\sigma_{k l}^{\mathrm{kin}}(\boldsymbol{r}, t) V_{l}(\boldsymbol{r}, t) \boldsymbol{e}_{\boldsymbol{k}}\right)
\end{aligned}
$$

The material derivative $D_{t}$ is defined as $D_{t}:=\partial / \partial t+\boldsymbol{V}(\boldsymbol{r}, t) \cdot \nabla_{\boldsymbol{r}}$. Therefore, we get

$$
\begin{aligned}
D_{t}\left[\frac{2}{d} \rho(\boldsymbol{r}, t) T(\boldsymbol{r}, t)\right]+D_{t}\left[\frac{m}{2} \rho(\boldsymbol{r}, t)|\boldsymbol{V}(\boldsymbol{r}, t)|^{2}\right]= & \frac{1}{\Delta t} \sum_{i=1}^{N}\left(\Delta E_{i}\right) \Phi\left(\boldsymbol{r}-\boldsymbol{r}_{\boldsymbol{i}}\right) \\
& -\frac{m}{2} \rho(\boldsymbol{r}, t)|\boldsymbol{V}(\boldsymbol{r}, t)|^{2} \nabla_{\boldsymbol{r}} \cdot \boldsymbol{V}(\boldsymbol{r}, t) \\
& -\nabla_{\boldsymbol{r}} \cdot \boldsymbol{q}^{\mathrm{kin}}(\boldsymbol{r}, t) \\
& -\rho(\boldsymbol{r}, t) T(\boldsymbol{r}, t) \nabla_{\boldsymbol{r}} \cdot \boldsymbol{V}(\boldsymbol{r}, t) \\
& -\nabla_{\boldsymbol{r}} \cdot\left(\sigma_{k l}^{\mathrm{kin}}(\boldsymbol{r}, t) V_{l}(\boldsymbol{r}, t) \boldsymbol{e}_{\boldsymbol{k}}\right)
\end{aligned}
$$

Using the mass balance equation $D_{t} \rho(\boldsymbol{r}, t)+\rho(\boldsymbol{r}, t) \nabla_{\boldsymbol{r}} \cdot \boldsymbol{V}(\boldsymbol{r}, t)=0$ this reduces to

$$
\begin{aligned}
\rho(\boldsymbol{r}, t) D_{t}\left(\frac{2}{d} T(\boldsymbol{r}, t)+\frac{m}{2}|\boldsymbol{V}(\boldsymbol{r}, t)|^{2}\right)= & \frac{1}{\Delta t} \sum_{i=1}^{N}\left(\Delta E_{i}\right) \Phi\left(\boldsymbol{r}-\boldsymbol{r}_{\boldsymbol{i}}\right) \\
& -\nabla_{\boldsymbol{r}} \cdot \boldsymbol{q}^{\mathrm{kin}}(\boldsymbol{r}, t) \\
& -\nabla_{\boldsymbol{r}} \cdot\left(\sigma_{k l}^{\mathrm{kin}}(\boldsymbol{r}, t) V_{l}(\boldsymbol{r}, t) \boldsymbol{e}_{\boldsymbol{k}}\right)
\end{aligned}
$$

Noting that $\sigma_{k l}^{\text {kin }}(\boldsymbol{r}, t)=\sigma_{k l}(\boldsymbol{r}, t)-\sigma_{k l}^{\text {int }}(\boldsymbol{r}, t)$ and defining

$$
\boldsymbol{q}^{\mathrm{int}_{\mathrm{v}}}(\boldsymbol{r}, t):=-\sigma_{k l}^{\mathrm{int}}(\boldsymbol{r}, t) V_{l}(\boldsymbol{r}, t) \boldsymbol{e}_{\boldsymbol{k}} \equiv-\boldsymbol{\sigma}^{\mathrm{int}}(\boldsymbol{r}, t) \cdot \boldsymbol{V}(\boldsymbol{r}, t)
$$

we write

$$
\begin{aligned}
\rho(\boldsymbol{r}, t) D_{t}\left(\frac{2}{d} T(\boldsymbol{r}, t)+\frac{m}{2}|\boldsymbol{V}(\boldsymbol{r}, t)|^{2}\right)= & \frac{1}{\Delta t} \sum_{i=1}^{N}\left(\Delta E_{i}\right) \Phi\left(\boldsymbol{r}-\boldsymbol{r}_{\boldsymbol{i}}\right) \\
& -\nabla_{\boldsymbol{r}} \cdot\left[\boldsymbol{q}^{\mathrm{kin}}(\boldsymbol{r}, t)+\boldsymbol{q}^{\mathrm{int}_{\mathrm{v}}}(\boldsymbol{r}, t)\right] \\
& -\nabla_{\boldsymbol{r}} \cdot\left(\sigma_{k l}(\boldsymbol{r}, t) V_{l}(\boldsymbol{r}, t) \boldsymbol{e}_{\boldsymbol{k}}\right)
\end{aligned}
$$


The last term of Eq. (A.8) is given by $-\partial_{k}\left(\sigma_{k l}(\boldsymbol{r}, t) V_{l}(\boldsymbol{r}, t)\right)=-\sigma_{k l}(\boldsymbol{r}, t) \partial_{k} V_{l}(\boldsymbol{r}, t)-$ $V_{l}(\boldsymbol{r}, t) \partial_{k} \sigma_{k l}(\boldsymbol{r}, t)$. Its last part can be transformed using the momentum balance equation, $m \rho(\boldsymbol{r}, t) D_{t} V_{l}(\boldsymbol{r}, t)+\partial_{k} \sigma_{k l}(\boldsymbol{r}, t)=0$ such that $-V_{l}(\boldsymbol{r}, t) \partial_{k} \sigma_{k l}(\boldsymbol{r}, t)=$ $m \rho(\boldsymbol{r}, t) V_{l}(\boldsymbol{r}, t) D_{t} V_{l}(\boldsymbol{r}, t)=\frac{m}{2} \rho(\boldsymbol{r}, t) D_{t}|\boldsymbol{V}(\boldsymbol{r}, t)|^{2}$.

Therefore, we get

$$
\begin{aligned}
\rho(\boldsymbol{r}, t) D_{t} T(\boldsymbol{r}, t)= & \frac{1}{\Delta t} \sum_{i=1}^{N}\left(\Delta E_{i}\right) \Phi\left(\boldsymbol{r}-\boldsymbol{r}_{\boldsymbol{i}}\right) \\
& -\nabla_{\boldsymbol{r}} \cdot\left[\boldsymbol{q}^{\mathrm{kin}}(\boldsymbol{r}, t)+\boldsymbol{q}^{\mathrm{int}_{\mathrm{v}}}(\boldsymbol{r}, t)\right] \\
& -\sigma_{k l}(\boldsymbol{r}, t) \partial_{k} V_{l}(\boldsymbol{r}, t)
\end{aligned}
$$

The term $\frac{1}{\Delta t} \sum_{i=1}^{N}\left(\Delta E_{i}\right) \Phi\left(\boldsymbol{r}-\boldsymbol{r}_{\boldsymbol{i}}\right)$ can be treated exactly as shown in Sec. 4.3.3. It yields the energy loss term $\zeta(\boldsymbol{r}, t)$, cf. Eq. (4.32), and the part of the collisional contribution to the heat flux $\boldsymbol{q}^{\text {into }}(\boldsymbol{r}, t)$, cf. Eq. (4.34), that present for zero-velocity fields, too. The result is given in Eqs. (4.36), (4.37), and (4.38). 


\section{B. Simple Energy-Balance Argument for Two Distinctively Driven Walls}

For two different walls with coefficients of restitution $\alpha_{\mathrm{w}_{1}}$ and $\alpha_{\mathrm{w}_{2}}$ driven by different driving velocities $v_{\mathrm{dr}_{1}}$ and $v_{\mathrm{dr}_{2}}$ a careful consideration of the two walls, analogous to Eq. (3.7), yields the very similar result

$$
\frac{d T}{d t}=\frac{m}{L_{x}} \sqrt{\frac{T}{2 \pi m}}\left(v_{\mathrm{dr}}^{2}+4 \widetilde{\alpha}_{\mathrm{w}} \sqrt{\frac{T}{2 \pi m}} v_{\mathrm{dr}}-\varepsilon_{\mathrm{eff}} \psi \frac{\pi}{2} \frac{T}{2 m}\right),
$$

where $\psi$ and $\varepsilon_{\text {eff }}$ have been introduced in Eqs. (3.9) and (3.8), respectively. Note that $\varepsilon_{\text {eff }}$ is a function of $\alpha_{\mathrm{w}}$, which will be defined in Eq. (B.2). Additionally $\widetilde{\alpha}_{\mathrm{w}}$ and $v_{\mathrm{dr}}$ have not been defined, yet. They are given by

$$
v_{\mathrm{dr}}^{2}:=\frac{v_{\mathrm{dr}_{1}}^{2}+v_{\mathrm{dr}_{2}}^{2}}{2} \geq 0, \quad \alpha_{\mathrm{w}}^{2}:=\frac{\alpha_{\mathrm{w}_{1}}^{2}+\alpha_{\mathrm{w}_{2}}^{2}}{2} \geq 0, \quad \widetilde{\alpha}_{\mathrm{w}} v_{\mathrm{dr}}:=\frac{\alpha_{\mathrm{w}_{1}} v_{\mathrm{dr}_{1}}+\alpha_{\mathrm{w}_{2}} v_{\mathrm{dr}_{2}}}{2} \geq 0 .
$$

With

$$
\psi_{\mathrm{eff}}:=\varepsilon_{\mathrm{eff}} \psi / \widetilde{\alpha}_{\mathrm{w}}
$$

the final result of the simple energy balance argument for the stationary state temperature with two distinct walls is

$$
T=\left(\frac{2}{\pi}\right)^{3} \frac{m v_{\mathrm{dr}}^{2}}{\psi_{\mathrm{eff}}^{2}}\left(1+\sqrt{1+(\pi / 2)^{2} \psi_{\mathrm{eff}} / \widetilde{\alpha}_{\mathrm{w}}}\right)^{2} .
$$

Of course, this equation simplifies to Eq. (3.12) for two identical walls and further simplifies to Eq. (3.13) if those identically driven walls are elastic. 


\section{Time Evolution of Energies Using a Pseudo-Liouville Operator Approach}

\section{C.1. Time Evolution in Terms of the Pseudo-Liouville Operator}

We show here in detail the derivation of the dynamic equations (10.4) with coefficients for model E, Eqs. (10.23) and (10.24), in chapter 10 as well as the refined balance equation (3.14) in chapter 3. Using a pseudo-Liouville operator approach we calculate differential equations for the $x$ - and $y$-component of the translational temperature as well as the (z-component of the) rotational temperature for the two-dimensional problem. The beginning of the calculations will be very general. Special cases will be implemented as late as possible in order to use the same calculation for different settings, e.g. free cooling, homogeneously driven and driven through the walls. For comparison we will cite the free cooling result of the $3 \mathrm{D}$ problem in Sec. C.2.3.

Let the vectors of position, translational and rotational velocity of a particle $k$, in a twodimensional plane $(x, y)$ with only vertical spin $(z)$, be defined as $\boldsymbol{r}_{k}=\left(r_{k, x}, r_{k, y}, 0\right)$, $\boldsymbol{v}_{k}=\left(v_{k, x}, v_{k, y}, 0\right)$, and $\boldsymbol{\omega}_{k}=\left(0,0, \omega_{k}\right)$.

The time evolution of a dynamic variable $A(t)$ that depends on time only through the positions and velocities of $N$ particles, can be determined by means of a pseudo-Liouville operator $\mathcal{L}_{+}$ for $t>0$

$$
A(t)=\exp \left(i \mathcal{L}_{+} t\right) A(0)
$$

The pseudo-Liouville operator $\mathcal{L}_{+}$can be decomposed into four parts $\mathcal{L}_{+}=\mathcal{L}_{0}+\mathcal{L}_{+}^{\mathrm{pp}}+\mathcal{L}_{+}^{\mathrm{pw}}+$ $\mathcal{L}_{+}^{\mathrm{H}_{\mathrm{dr}}}$. The first one, $\mathcal{L}_{0}$ describes the free streaming of particles

$$
\mathcal{L}_{0}=-i \sum_{k=1}^{N} \boldsymbol{v}_{k} \cdot \nabla_{\boldsymbol{r}_{k}},
$$

the second and third one describe hard-core particle-particle (pp) and particle-wall (pw) collisions. Since the wall can be interpreted as an infinitely large particle at a fixed position, those 
two parts are very similar. Finally, $\mathcal{L}_{+}^{\mathrm{H}_{\mathrm{dr}}}$, can be used to model any type of homogeneous driving, e.g. stochastic driving as used in chapter 10.

The particle-particle contribution $\mathcal{L}_{+}^{\text {pp }}$ is a sum over all possible pairs of particles $(k, l)$,

$$
\begin{aligned}
\mathcal{L}_{+}^{\mathrm{pp}} & =\frac{1}{2} \sum_{l \neq k} T_{+}^{k l}, \text { where } \\
T_{+}^{k l} & =i\left(\boldsymbol{v}_{k l} \cdot \hat{\boldsymbol{r}}_{k l}\right) \Theta\left(-\boldsymbol{v}_{k l} \cdot \hat{\boldsymbol{r}}_{k l}\right) \delta\left(\left|\boldsymbol{r}_{k l}\right|-a\right)\left(b_{+}^{k l}-1\right)
\end{aligned}
$$

describes hard-core collisions between particles $k$ and $l$. The operator $b_{+}^{k l}$ replaces the linear and angular momenta of the two particles $k$ and $l$ before collision by the corresponding ones after collision, according to Eqs. (2.4) and (2.5). $\Theta(x)$ is the Heaviside step-function, and we have introduced the notation $\boldsymbol{r}_{k l}=\boldsymbol{r}_{k}-\boldsymbol{r}_{l}$ and $\hat{\boldsymbol{r}}_{k l}=\boldsymbol{r}_{k l} /\left|\boldsymbol{r}_{k l}\right|$. Equation (C.3) has the following interpretation. The factor $\boldsymbol{v}_{k l} \cdot \hat{\boldsymbol{r}}_{k l}$ gives the flux of incoming particles. The $\Theta$ - and $\delta$-functions specify the conditions for a collision to take place. A collision between particles $k$ and $l$ happens only if the two particles are approaching each other which is ensured by $\Theta\left(-\boldsymbol{v}_{k l} \cdot \hat{\boldsymbol{r}}_{k l}\right)$. At the instant of a collision the distance between the two particles has to vanish when two particles touch, which is expressed by $\delta\left(\left|\boldsymbol{r}_{k l}\right|-a\right)$. Finally, $\left(b_{+}^{k l}-1\right)$ generates the change of linear and angular momenta according to Eqs. (2.4) and (2.5).

The particle-wall contribution is a sum over all possible pairs of one particle and one wall, where the sum of $l$ runs from 1 to the number of walls in the system $N_{W}$,

$$
\begin{aligned}
\mathcal{L}_{+}^{\mathrm{pw}} & =\sum_{l=1}^{N_{W}} \sum_{k=1}^{N} T_{+}^{W_{l} k}, \text { where } \\
T_{+}^{W_{l} k} & =i\left(\hat{\boldsymbol{n}}_{l} \cdot \boldsymbol{v}_{k}\right) \Theta\left(-\hat{\boldsymbol{n}}_{l} \cdot \boldsymbol{v}_{k}\right) \delta\left(\left|\hat{\boldsymbol{n}}_{l} \cdot\left(\boldsymbol{r}_{k}-\boldsymbol{w}_{l}\right)\right|-\frac{a}{2}\right)\left(b_{+}^{W_{l} k}-1\right),
\end{aligned}
$$

where $\hat{\boldsymbol{n}}_{l}$ is the normal vector of wall $l$ pointing into the system and $\boldsymbol{w}_{l}$ some point of wall $l$. $\left(b_{+}^{W_{l} k}-1\right)$ generates the change of linear and angular momentum of particle $k$ according to the particle-wall collision rule (2.17).

The ensemble average, $\langle\ldots\rangle_{t}$, of a dynamic variable, $A$, is defined by

$$
\begin{aligned}
\langle A\rangle_{t} & =\int d \Gamma \rho(0) A(t)=\int d \Gamma \rho(t) A(0) \\
& =\int \prod_{k=1}^{N}\left(d^{2} r_{k} d^{2} v_{k} d \omega_{k}\right) \rho(t) A(0) .
\end{aligned}
$$


Here $\rho(t)=\exp \left(-i \mathcal{L}_{+}^{\dagger} t\right) \rho(0)$ is the $N$-particle distribution function, whose time development is governed by the adjoint $\mathcal{L}_{+}^{\dagger}$ of the time evolution operator $\mathcal{L}_{+}$. Differentiating equation (C.5) with respect to time yields

$$
\begin{aligned}
\frac{d}{d t}\langle A\rangle_{t} & =\int d \Gamma \rho(0) \frac{d}{d t} A(t)=\int d \Gamma \rho(0) i \mathcal{L}_{+} A(t) \\
& =\int d \Gamma \rho(0) \exp \left(i \mathcal{L}_{+} t\right) i \mathcal{L}_{+} A(0) \\
& =\int d \Gamma \rho(t) i \mathcal{L}_{+} A(0)=\left\langle i \mathcal{L}_{+} A\right\rangle_{t}
\end{aligned}
$$

We are interested in the following averaged energies per particle

$$
\begin{gathered}
\frac{1}{2} T_{\mathrm{x} / \mathrm{y}}:=E_{\mathrm{x} / \mathrm{y}}=\frac{1}{N} \sum_{i=1}^{N} \frac{m}{2} v_{i, x / y}^{2} \\
\frac{1}{2} T_{\mathrm{rot}}:=E_{\mathrm{rot}}=\frac{1}{N} \sum_{i=1}^{N} \frac{I}{2} \omega_{i, z}^{2}
\end{gathered}
$$

as well as the total translational energy $E_{\mathrm{tr}}=E_{\mathrm{x}}+E_{\mathrm{y}}$ and the total kinetic energy $E=$ $E_{\mathrm{tr}}+E_{\mathrm{rot}}$. If the system is invariant under exchange of $x$ and $y, T_{\mathrm{x}}=T_{\mathrm{y}}=T_{\mathrm{tr}}$. This is the case for a homogeneously heated system without walls like in chapter 10 .

Assuming a homogeneous density distribution and Gaussian velocity distributions the $N$ particle distribution function is given by

$$
\rho(t) \propto \prod_{k<l} \Theta\left(\left|\boldsymbol{r}_{k l}\right|-a\right) \exp \left\{-\left(\frac{E_{\mathrm{x}}}{T_{\mathrm{x}}(t)}+\frac{E_{\mathrm{y}}}{T_{\mathrm{y}}(t)}+\frac{E_{\mathrm{rot}}}{T_{\mathrm{rot}}(t)}\right)\right\}
$$

where the product of Heaviside functions accounts for the excluded area (volume). Hence we get three coupled differential equations for the time evolution of the translational and rotational energies. Additionally, we restrict the homogeneous driving to the translational degrees of freedom and get

$$
\begin{aligned}
& \frac{1}{2} \frac{d}{d t} T_{\mathrm{x} / \mathrm{y}}(t)=\frac{d}{d t}\left\langle E_{\mathrm{x} / \mathrm{y}}\right\rangle_{t}=\left\langle i \mathcal{L}_{+} E_{\mathrm{x} / \mathrm{y}}\right\rangle_{t}=H_{\mathrm{dr}}+\left\langle i \mathcal{L}_{+}^{\mathrm{pp}} E_{\mathrm{x} / \mathrm{y}}+\left\langle i \mathcal{L}_{+}^{\mathrm{pw}} E_{\mathrm{x} / \mathrm{y}}\right\rangle_{t}\right. \\
& \frac{1}{2} \frac{d}{d t} T_{\mathrm{rot}}(t)=\frac{d}{d t}\left\langle E_{\mathrm{rot}}\right\rangle_{t}=\left\langle i \mathcal{L}_{+} E_{\mathrm{rot}}\right\rangle_{t}=\left\langle i \mathcal{L}_{+}^{\mathrm{pp}} E_{\mathrm{rot}}\right\rangle_{t}+\left\langle i \mathcal{L}_{+}^{\mathrm{pw}} E_{\mathrm{rot}}\right\rangle_{t}
\end{aligned}
$$

where $i \mathcal{L}_{+}^{\mathrm{pp}}$ is the pseudo-Liouville collision operator for particle-particle collisions and $i \mathcal{L}_{+}^{\mathrm{pw}}$ is the pseudo-Liouville operator for particle-wall collisions as defined above. $H_{\mathrm{dr}}=f_{\mathrm{dr}} T_{\mathrm{dr}}$ is the homogeneous driving term where $f_{\mathrm{dr}}$ is the driving frequency and $T_{\mathrm{dr}}$ is the driving temperature. For a homogeneously driven system, $T_{\mathrm{x}}=T_{\mathrm{y}}=T_{\mathrm{tr}}$. In that case we can replace the $\mathrm{x} / \mathrm{y}$ by tr but have to multiply the left hand side by two because $T_{\mathrm{tr}}$ describes two degrees of freedom. 


\section{C.2. Particle-Particle Contribution $\left\langle i \mathcal{L}_{+}^{\mathrm{pp}} E_{\mathrm{x} / \mathrm{y} / \mathrm{tr} / \mathrm{rot}}\right\rangle_{t}$ to}

$$
\frac{d}{d t} T_{\mathrm{x} / \mathrm{y} / \mathrm{tr} / \operatorname{rot}}(t)
$$

To minimize the number of indices we show here the particle-particle contribution of the time derivative of $T_{\mathrm{x} / \mathrm{y}}$. For $T_{\mathrm{tr}}$ we only have to add the $x$ - and $y$-contributions and divide by two. For $T_{\text {rot }}$ nothing changes except $\Delta E_{\mathrm{x} / \mathrm{y}}$ [as later given in Eq. (C.15)] has to be replaced by $\Delta E_{\text {rot }}$ [as later given in Eq. (C.53)] and, of course, in the very next equation (C.10) we need to replace the $\left|v_{n, x / y}\right|^{2}$ by $\left|\omega_{n}\right|^{2}$. The particle-particle contribution to the time derivative of $T_{\mathrm{x} / \mathrm{y}}$ is given by

$$
\begin{aligned}
\left\langle i \mathcal{L}_{+}^{\mathrm{pp}} E_{\mathrm{x} / \mathrm{y}}\right\rangle_{t} & \stackrel{(*)}{=}\left\langle i \mathcal{L}_{U+} E_{\mathrm{x} / \mathrm{y}}\right\rangle_{t}=\left\langle\frac{1}{2} \sum_{k \neq l} i T_{+}^{k l} E_{\mathrm{x} / \mathrm{y}}\right\rangle_{t}=\left\langle\frac{1}{2} \sum_{k=1}^{N} \sum_{\substack{l=1 \\
l \neq k}}^{N} i T_{+}^{k l} E_{\mathrm{x} / \mathrm{y}}\right\rangle_{t} \\
& =\left\langle\frac{1}{2} \sum_{k=1}^{N} \sum_{\substack{l=1 \\
l \neq k}}^{N} i T_{+}^{k l} \frac{1}{N} \sum_{n=1}^{N} \frac{m}{2}\left|v_{n, x / y}\right|^{2}\right\rangle_{t} \stackrel{(* *)}{=} \frac{1}{2 N}\left\langle\sum_{k=1}^{N} \sum_{\substack{l=1 \\
l \neq k}}^{N} \frac{m}{2} i T_{+}^{k l}\left(\left|v_{k, x / y}\right|^{2}+\left|v_{l, x / y}\right|^{2}\right)\right\rangle_{t} \\
& =\frac{1}{2 N} \sum_{k=1}^{N} \sum_{\substack{l=1 \\
l \neq k}}^{N} \frac{m}{2} \int \prod_{n=1}^{N} d r_{j, x} d r_{j, y} d v_{j, x} d v_{j, y} d \omega_{j} \rho(\boldsymbol{r}, \boldsymbol{v}, \boldsymbol{\omega}) i T_{+}^{k l}\left(\left|v_{k, x / y}\right|^{2}+\left|v_{l, x / y}\right|^{2}\right) \\
& \stackrel{(* * *)}{=} \frac{1}{2 N} N(N-1) \int \prod_{n=1}^{N} d^{2} r_{j} d^{2} v_{j} d \omega_{j} \rho(\boldsymbol{r}, \boldsymbol{v}, \boldsymbol{\omega}) i T_{+}^{12} \frac{m}{2}\left(\left|v_{1, x / y}\right|^{2}+\left|v_{2, x / y}\right|^{2}\right)
\end{aligned}
$$

The equality $(*)$ holds because the free streaming of particles yields no change in energy. $(* *)$ holds because $i T_{+}^{k l}\left|v_{n, x / y}\right|^{2}=0$ if $n \neq k$ and $n \neq l$. Finally, $(* * *)$ is true because this distribution function $\rho$ (Maxwell-Boltzmann distribution) is invariant under permutation of the components. Defining

$$
d \Gamma:=\prod_{j=1}^{N} d^{2} r_{j} d^{2} v_{j} d \omega_{j} \prod_{l \neq j} \Theta\left(\left|\boldsymbol{r}_{j l}\right|-a\right) \exp \left(-\sum_{k=1}^{N}\left[\frac{m v_{k, x}^{2}}{2 T_{\mathrm{x}}(t)}+\frac{m v_{k, y}^{2}}{2 T_{\mathrm{y}}(t)}+\frac{I \omega_{k}^{2}}{2 T_{\mathrm{rot}}(t)}\right]\right)
$$

and using the definition of $i T_{+}^{12}$, Eq. (C.3), we can write

$$
\left\langle i \mathcal{L}_{+}^{\mathrm{pp}} E_{\mathrm{x} / \mathrm{y}}\right\rangle_{t}=-\frac{N-1}{2 \int d \Gamma} \int d \Gamma\left(\hat{\boldsymbol{r}}_{12} \cdot \boldsymbol{v}_{12}\right) \delta\left(\left|\boldsymbol{r}_{12}\right|-a\right) \Theta\left(-\hat{\boldsymbol{r}}_{12} \cdot \hat{\boldsymbol{v}}_{12}\right) \Delta E_{\mathrm{x} / \mathrm{y}} \cdot
$$

The change of partial energy $\Delta E_{\mathrm{x} / \mathrm{y}}:=\frac{m}{2}\left(b_{+}^{12}-1\right)\left(v_{1, x / y}^{2}+v_{2, x / y}^{2}\right)$ that results from a collision of particle 1 and 2 depends only on the phase space variables of particle 1 and 2. Since we assume spatial homogeneity this change of energy can only depend on the relative distance vector $\boldsymbol{r}_{12}=\boldsymbol{r}_{1}-\boldsymbol{r}_{2}$ as well as the relative translational and rotational velocities $\boldsymbol{v}_{12}=\boldsymbol{v}_{1}-\boldsymbol{v}_{2}$ and $\boldsymbol{\omega}_{12}=\boldsymbol{\omega}_{1}+\boldsymbol{\omega}_{2}$. Further, we assume instantaneous collisions. Therefore 
the change of energy can only depend on the direction of the distance vector $\hat{\boldsymbol{r}}_{12}=\frac{\boldsymbol{r}_{1}-\boldsymbol{r}_{2}}{\left|\boldsymbol{r}_{1}-\boldsymbol{r}_{2}\right|}$. Now we can perform the integrations over $d^{2} r_{3} \ldots d^{2} r_{N}, d^{2} v_{3} \ldots d^{2} v_{N}$ and $d \omega_{3} \ldots d \omega_{N}$. The integration over $d^{2} v_{3} \ldots d^{2} v_{N}$ and $d^{2} \omega_{3} \ldots d^{2} \omega_{N}$ are simple Gaussian integrals. To integrate over $d^{2} r_{3} \ldots d^{2} r_{N}$ we introduce two more two-dimensional integrals over two-dimensional $\delta$ functions, $\delta^{2}(\boldsymbol{r}):=\delta\left(r_{x}\right) \delta\left(r_{y}\right), \int d^{2} R_{1} d^{2} R_{2} \delta^{2}\left(\boldsymbol{R}_{1}-\boldsymbol{r}_{1}\right) \delta^{2}\left(\boldsymbol{R}_{2}-\boldsymbol{r}_{2}\right)$. The pair correlation function is defined as

$$
\frac{g\left(\left|\boldsymbol{R}_{1}-\boldsymbol{R}_{2}\right|\right)}{V^{2}}:=\frac{\int \prod_{j=1}^{N} d^{2} r_{j} \prod_{l \neq j} \Theta\left(\left|\boldsymbol{r}_{j l}\right|-a\right) \delta^{2}\left(\boldsymbol{R}_{1}-\boldsymbol{r}_{1}\right) \delta^{2}\left(\boldsymbol{R}_{2}-\boldsymbol{r}_{2}\right)}{\int \prod_{j=1}^{N} d^{2} r_{j} \prod_{l \neq j} \Theta\left(\left|\boldsymbol{r}_{j l}\right|-a\right)},
$$

where $V$ is the area of the system. Using $\boldsymbol{R}_{12}:=\boldsymbol{R}_{1}-\boldsymbol{R}_{2}$ and not writing down the explicit time dependence of the temperatures any more we obtain

$$
\begin{gathered}
\left\langle i \mathcal{L}_{+}^{\mathrm{pp}} E_{\mathrm{x} / \mathrm{y}}\right\rangle_{t}=-\frac{N-1}{2 V^{2}}\left(\frac{m}{2 \pi T_{\mathrm{x}}}\right)\left(\frac{m}{2 \pi T_{\mathrm{y}}}\right)\left(\frac{I}{2 \pi T_{\mathrm{rot}}}\right) \int d^{2} R_{1} d^{2} R_{2} d^{2} v_{1} d^{2} v_{2} d^{2} \omega_{1} d \omega_{2} \times \\
g\left(\left|\boldsymbol{R}_{12}\right|\right) \exp \left(-\frac{m}{2}\left[\frac{v_{1, x}^{2}+v_{2, x}^{2}}{T_{\mathrm{x}}}+\frac{v_{1, y}^{2}+v_{2, y}^{2}}{T_{\mathrm{y}}}\right]-\frac{I}{2} \frac{\omega_{1}^{2}+\omega_{2}^{2}}{T_{\mathrm{rot}}}\right) \times \\
\left(\hat{\boldsymbol{R}}_{12} \cdot \boldsymbol{v}_{12}\right) \delta\left(\left|\boldsymbol{R}_{12}\right|-a\right) \Theta\left(-\hat{\boldsymbol{R}}_{12} \cdot \hat{\boldsymbol{v}}_{12}\right) \Delta E_{\mathrm{x} / \mathrm{y}} .
\end{gathered}
$$

Since the change of energy $\Delta E_{\mathrm{x} / \mathrm{y}}$ depends only on $\boldsymbol{R}_{12}=\boldsymbol{R}_{1}-\boldsymbol{R}_{2}, \boldsymbol{v}_{12}=\boldsymbol{v}_{1}-\boldsymbol{v}_{2}$, $\boldsymbol{\omega}_{12}=\boldsymbol{\omega}_{1}+\boldsymbol{\omega}_{2}$ we introduce the variables

$$
\begin{aligned}
\boldsymbol{r} & :=\boldsymbol{R}_{1}-\boldsymbol{R}_{2}, \quad \boldsymbol{v}:=\frac{\boldsymbol{v}_{1}-\boldsymbol{v}_{2}}{\sqrt{2}}, \quad \boldsymbol{\omega}:=\frac{\boldsymbol{\omega}_{1}+\boldsymbol{\omega}_{2}}{\sqrt{2}} \\
\boldsymbol{R} & :=\boldsymbol{R}_{1}, \quad \boldsymbol{V}:=\frac{\boldsymbol{v}_{1}+\boldsymbol{v}_{2}}{\sqrt{2}}, \quad \boldsymbol{\Omega}:=\frac{\boldsymbol{\omega}_{1}-\boldsymbol{\omega}_{2}}{\sqrt{2}} .
\end{aligned}
$$

The Jacobian of this transformation is 1. The expression to integrate over is independent of $\boldsymbol{R}$ such that integration over $d^{2} R$ yields the area $V$. We write $\boldsymbol{r}=r \hat{\boldsymbol{r}}$ in polar coordinates $\left(r, \phi_{r}\right)$ and can integrate over $d r$. The integrals over $d^{2} V$ and $d \Omega$ are plain Gaussians. We obtain

$$
\begin{aligned}
\left\langle i \mathcal{L}_{+}^{\mathrm{pp}} E_{\mathrm{x} / \mathrm{y}}\right\rangle_{t}=- & \frac{1}{\sqrt{2}} a n_{0} g(a)\left(\frac{m}{2 \pi T_{\mathrm{x}}}\right)^{\frac{1}{2}}\left(\frac{m}{2 \pi T_{\mathrm{y}}}\right)^{\frac{1}{2}}\left(\frac{I}{2 \pi T_{\mathrm{rot}}}\right)^{\frac{1}{2}} \int d \phi_{r} d v_{x} d v_{y} d \omega \times \\
& (\hat{\boldsymbol{r}} \cdot \boldsymbol{v}) \Theta(-\hat{\boldsymbol{r}} \cdot \hat{\boldsymbol{v}}) \exp \left(\frac{-\frac{m}{2} v_{x}^{2}}{T_{\mathrm{x}}}+\frac{-\frac{m}{2} v_{y}^{2}}{T_{\mathrm{y}}}+\frac{-\frac{I}{2} \omega^{2}}{T_{\mathrm{rot}}}\right) \Delta E_{\mathrm{x} / \mathrm{y}} .
\end{aligned}
$$

with the number density $n_{0}:=(N-1) / V \approx N / V$. 


\section{C.2.1. PP Contribution $\left\langle i \mathcal{L}_{+}^{\mathrm{pp}} E_{\mathrm{x} / \mathrm{y}}\right\rangle_{t}$ to the Change of Translational Energy}

To solve the integrals above we need to take a look at the change of energy $\Delta E_{\mathrm{x} / \mathrm{y}}(\hat{\boldsymbol{r}}, \boldsymbol{v}, \boldsymbol{\omega})$ :

$$
\begin{aligned}
& \frac{2}{m} \Delta E_{\mathrm{x} / \mathrm{y}}:=\left(b_{+}^{12}-1\right)\left(\left|v_{1, x / y}\right|^{2}+\left|v_{2, x / y}\right|^{2}\right)=\left(\left|v_{1, x / y}^{\prime}\right|^{2}+\left|v_{2, x / y}^{\prime}\right|^{2}\right)-\left(\left|v_{1, x / y}\right|^{2}+\left|v_{2, x / y}\right|^{2}\right) \\
& \stackrel{(2.4)}{=}\left(\left|v_{1, x / y}+\Delta v_{x / y}\right|^{2}+\left|v_{2, x / y}-\Delta v_{x / y}\right|^{2}\right)-\left(\left|v_{1, x / y}\right|^{2}+\left|v_{2, x / y}\right|^{2}\right) \\
&= 2\left|\Delta v_{x / y}\right|^{2}+2 v_{12, x / y} \Delta v_{x / y} \\
&\left(\stackrel{(2.5)}{=} 2\left(\frac{1+\alpha}{2}\right)^{2}\left(\hat{\boldsymbol{r}}_{12} \cdot \boldsymbol{v}_{12}\right)^{2}\left(\hat{\boldsymbol{r}}_{12} \cdot \hat{\boldsymbol{e}}_{x / y}\right)^{2}\right. \\
&+2 \eta^{2}\left[\left(\boldsymbol{v}_{12}-\hat{\boldsymbol{r}}_{12}\left[\hat{\boldsymbol{r}}_{12} \cdot \boldsymbol{v}_{12}\right]+\left(\frac{a}{2}\right)\left[\hat{\boldsymbol{r}}_{12} \times \boldsymbol{\omega}_{12}\right]\right) \cdot \hat{\boldsymbol{e}}_{x / y}\right]^{2} \\
&+2 \eta(1+\alpha)\left(\hat{\boldsymbol{r}}_{12} \cdot \boldsymbol{v}_{12}\right)\left(\hat{\boldsymbol{r}}_{12} \cdot \hat{\boldsymbol{e}}_{x / y}\right)\left[\left(\boldsymbol{v}_{12}-\hat{\boldsymbol{r}}_{12}\left[\hat{\boldsymbol{r}}_{12} \cdot \boldsymbol{v}_{12}\right]+\left(\frac{a}{2}\right)\left[\hat{\boldsymbol{r}}_{12} \times \boldsymbol{\omega}_{12}\right]\right) \cdot \hat{\boldsymbol{e}}_{x / y}\right] \\
&-(1+\alpha)\left(\hat{\boldsymbol{r}}_{12} \cdot \boldsymbol{v}_{12}\right)\left(\hat{\boldsymbol{r}}_{12} \cdot \hat{\boldsymbol{e}}_{x / y}\right)\left(\boldsymbol{v}_{12} \cdot \hat{\boldsymbol{e}}_{x / y}\right) \\
&-2 \eta\left[\left(\boldsymbol{v}_{12}-\hat{\boldsymbol{r}}_{12}\left[\hat{\boldsymbol{r}}_{12} \cdot \boldsymbol{v}_{12}\right]+\left(\frac{a}{2}\right)\left[\hat{\boldsymbol{r}}_{12} \times \boldsymbol{\omega}_{12}\right]\right) \cdot \hat{\boldsymbol{e}}_{x / y}\right]\left(\boldsymbol{v}_{12} \cdot \hat{\boldsymbol{e}}_{x / y}\right) \\
&=(1+\alpha)^{2}(\hat{\boldsymbol{r}} \cdot \boldsymbol{v})^{2}\left(\hat{\boldsymbol{r}} \cdot \hat{\boldsymbol{e}}_{x / y}\right)^{2}+4 \eta^{2}\left[\left(\boldsymbol{v}-\hat{\boldsymbol{r}}[\hat{\boldsymbol{r}} \cdot \boldsymbol{v}]+\left(\frac{a}{2}\right)[\hat{\boldsymbol{r}} \times \boldsymbol{\omega}]\right) \cdot \hat{\boldsymbol{e}}_{x / y}\right]^{2} \\
&+4 \eta(1+\alpha)(\hat{\boldsymbol{r}} \cdot \boldsymbol{v})\left(\hat{\boldsymbol{r}} \cdot \hat{\boldsymbol{e}}_{x / y}\right)\left[\left(\boldsymbol{v}-\hat{\boldsymbol{r}}[\hat{\boldsymbol{r}} \cdot \boldsymbol{v}]+\left(\frac{a}{2}\right)[\hat{\boldsymbol{r}} \times \boldsymbol{\omega}]\right) \cdot \hat{\boldsymbol{e}}_{x / y}\right] \\
&-2(1+\alpha)(\hat{\boldsymbol{r}} \cdot \boldsymbol{v})\left(\hat{\boldsymbol{r}} \cdot \hat{\boldsymbol{e}}_{x / y}\right)\left(\boldsymbol{v} \cdot \hat{\boldsymbol{e}}_{x / y}\right) \\
&-4 \eta\left[\left(\boldsymbol{v}-\hat{\boldsymbol{r}}[\hat{\boldsymbol{r}} \cdot \boldsymbol{v}]+\left(\frac{a}{2}\right)[\hat{\boldsymbol{r}} \times \boldsymbol{\omega}]\right) \cdot \hat{\boldsymbol{e}}_{x / y}\right]\left(\boldsymbol{v} \cdot \hat{\boldsymbol{e}}_{x / y}\right)
\end{aligned}
$$

where, again, $\boldsymbol{v}_{12}:=\boldsymbol{v}_{\mathbf{1}}-\boldsymbol{v}_{\mathbf{2}}, \boldsymbol{\omega}_{12}:=\boldsymbol{\omega}_{\mathbf{1}}+\boldsymbol{\omega}_{\mathbf{2}}$ and $\eta=\frac{q}{1+q} \frac{1+\beta}{2}$ with $\beta=\beta(\gamma) \stackrel{(2.8)}{=}$ const $\min \left(|\cot \gamma|,\left|\cot \gamma_{0}\right|\right)$ being a function of $|\cot \gamma|:=\frac{|\boldsymbol{g} \cdot \hat{\boldsymbol{r}}|}{|\boldsymbol{g} \times \hat{\boldsymbol{r}}|}$ and $\boldsymbol{g} \stackrel{(2.1)}{=} \boldsymbol{v}_{12}+\frac{a}{2} \hat{\boldsymbol{r}}_{12} \times$ $\boldsymbol{\omega}_{12}=\sqrt{2}\left(\boldsymbol{v}+\frac{a}{2} \hat{\boldsymbol{r}} \times \boldsymbol{\omega}\right)$. Annotation: It can be checked easily that

$$
\begin{aligned}
\frac{2}{m} \Delta E_{\mathrm{tr}} & =\frac{2}{m}\left(\Delta E_{\mathrm{x}}+\Delta E_{\mathrm{y}}\right) \\
= & -\left(1-\alpha^{2}\right)(\hat{\boldsymbol{r}} \cdot \boldsymbol{v})^{2}+4 \eta(\eta-1)\left(|\boldsymbol{v}|^{2}-(\hat{\boldsymbol{r}} \cdot \boldsymbol{v})^{2}\right)+(a \eta)^{2}|\hat{\boldsymbol{r}} \times \boldsymbol{\omega}|^{2} \\
& +2 a \eta(2 \eta-1)(\hat{\boldsymbol{r}} \times \boldsymbol{\omega}) \cdot \boldsymbol{v}
\end{aligned}
$$

as usual. In that case we could easily integrate over $d \phi_{r}$ to yield $2 \pi$ because we could freely choose the coordinate system for integrating over $\boldsymbol{v}$. Calculating $\left\langle i \mathcal{L}_{+}^{\mathrm{pp}} E_{\mathrm{x}}\right\rangle_{t}$ and $\left\langle i \mathcal{L}_{+}^{\mathrm{pp}} E_{\mathrm{y}}\right\rangle_{t}$ separately requires some extra work because our coordinate systems are fixed. Because it 
does no harm to postpone the integration over $d \phi_{r}$ - not even for the case of $\Delta E_{\mathrm{tr}}$ - we first substitute

$$
\begin{aligned}
\boldsymbol{\omega}_{\perp} & :=\sqrt{2}(\hat{\boldsymbol{r}} \times \boldsymbol{\omega}) \\
\boldsymbol{g} & :=\sqrt{2}\left(\boldsymbol{v}+\frac{a}{2}(\hat{\boldsymbol{r}} \times \boldsymbol{\omega})\right)=\sqrt{2} \boldsymbol{v}+\frac{a}{2} \boldsymbol{\omega}_{\perp},
\end{aligned}
$$

The Jacobian of this substitution is $1 / 2^{3 / 2}$. Eq. (C.14) becomes

$$
\begin{aligned}
\left\langle i \mathcal{L}_{+}^{\mathrm{pp}} E_{\mathrm{x} / \mathrm{y}}\right\rangle_{t}= & -\frac{1}{2 \pi^{3 / 2}} a n_{0} g(a)\left(\frac{m}{4 T_{\mathrm{x}}}\right)^{\frac{1}{2}}\left(\frac{m}{4 T_{\mathrm{y}}}\right)^{\frac{1}{2}}\left(\frac{I}{4 T_{\mathrm{rot}}}\right)^{\frac{1}{2}} \int d \phi_{r} d g_{x} d g_{y} d \omega_{\perp}(\hat{\boldsymbol{r}} \cdot \boldsymbol{g}) \Theta(-\hat{\boldsymbol{r}} \cdot \hat{\boldsymbol{g}}) \times \\
& \exp \left(-\frac{m\left[\left(\boldsymbol{g}-\frac{a}{2} \boldsymbol{\omega}_{\perp}\right) \cdot \hat{\boldsymbol{e}}_{x}\right]^{2}}{4 T_{\mathrm{x}}}-\frac{m\left[\left(\boldsymbol{g}-\frac{a}{2} \boldsymbol{\omega}_{\perp}\right) \cdot \hat{\boldsymbol{e}}_{y}\right]^{2}}{4 T_{\mathrm{y}}}-\frac{I\left|\boldsymbol{\omega}_{\perp}\right|^{2}}{4 T_{\mathrm{rot}}}\right) \Delta E_{\mathrm{x} / \mathrm{y}}
\end{aligned}
$$

where the change of partial energy reads

$$
\begin{aligned}
\frac{2}{m} \Delta E_{\mathrm{x} / \mathrm{y}}= & \frac{(1+\alpha)^{2}}{2}(\hat{\boldsymbol{r}} \cdot \boldsymbol{g})^{2}\left(\hat{\boldsymbol{r}} \cdot \hat{\boldsymbol{e}}_{x / y}\right)^{2}+2 \eta^{2}\left[(\boldsymbol{g}-\hat{\boldsymbol{r}}[\hat{\boldsymbol{r}} \cdot \boldsymbol{g}]) \cdot \hat{\boldsymbol{e}}_{x / y}\right]^{2} \\
& +2 \eta(1+\alpha)(\hat{\boldsymbol{r}} \cdot \boldsymbol{g})\left(\hat{\boldsymbol{r}} \cdot \hat{\boldsymbol{e}}_{x / y}\right)\left[(\boldsymbol{g}-\hat{\boldsymbol{r}}[\hat{\boldsymbol{r}} \cdot \boldsymbol{g}]) \cdot \hat{\boldsymbol{e}}_{x / y}\right] \\
& -(1+\alpha)(\hat{\boldsymbol{r}} \cdot \boldsymbol{g})\left(\hat{\boldsymbol{r}} \cdot \hat{\boldsymbol{e}}_{x / y}\right)\left(\left(\boldsymbol{g}-\frac{a}{2} \boldsymbol{\omega}_{\perp}\right) \cdot \hat{\boldsymbol{e}}_{x / y}\right) \\
& -2 \eta\left[(\boldsymbol{g}-\hat{\boldsymbol{r}}[\hat{\boldsymbol{r}} \cdot \boldsymbol{g}]) \cdot \hat{\boldsymbol{e}}_{x / y}\right]\left(\left(\boldsymbol{g}-\frac{a}{2} \boldsymbol{\omega}_{\perp}\right) \cdot \hat{\boldsymbol{e}}_{x / y}\right) .
\end{aligned}
$$

Again, it can be easily checked that

$$
\begin{aligned}
\frac{2}{m} \Delta E_{\mathrm{tr}} & =\frac{2}{m}\left(\Delta E_{\mathrm{x}}+\Delta E_{\mathrm{y}}\right) \\
& =-\frac{\left(1-\alpha^{2}\right)}{2}(\hat{\boldsymbol{r}} \cdot \boldsymbol{g})^{2}+2 \eta(\eta-1)\left(|\boldsymbol{g}|^{2}-(\hat{\boldsymbol{r}} \cdot \boldsymbol{g})^{2}\right)+a \eta \boldsymbol{g} \cdot \boldsymbol{\omega}_{\perp}
\end{aligned}
$$

Now we express $\hat{\boldsymbol{r}}$ and $\boldsymbol{g}$ in polar coordinates such that the angle between $\hat{\boldsymbol{r}}$ and $\boldsymbol{g}$ is called $\gamma$ and the angle between $\hat{\boldsymbol{r}}$ and $\boldsymbol{e}_{x}$ is called $\phi_{r}, \hat{\boldsymbol{r}} \rightarrow \phi_{r}(\hat{\boldsymbol{r}}$ is already normalized) and $\boldsymbol{g} \rightarrow(g, \gamma)$. Remembering that $\boldsymbol{\omega}_{\perp} \propto \hat{\boldsymbol{r}} \times \boldsymbol{\omega}$ and $\boldsymbol{\omega} \cdot \hat{\boldsymbol{r}}=0$ we get:

$$
\begin{aligned}
\hat{\boldsymbol{r}} \cdot \hat{\boldsymbol{e}}_{x}=\cos \left(\phi_{r}\right), & \hat{\boldsymbol{r}} \cdot \hat{\boldsymbol{e}}_{y}=\sin \left(\phi_{r}\right) \\
\hat{\boldsymbol{r}} \cdot \boldsymbol{g}=g \cos \gamma, & \boldsymbol{g} \cdot \boldsymbol{\omega}=g \omega_{\perp} \sin \gamma \\
\boldsymbol{g} \cdot \hat{\boldsymbol{e}}_{x}=g \cos \left(\phi_{r}-\gamma\right), & \boldsymbol{g} \cdot \hat{\boldsymbol{e}}_{y}=g \sin \left(\phi_{r}-\gamma\right) \\
\boldsymbol{\omega}_{\perp} \cdot \hat{\boldsymbol{e}}_{x}=\omega_{\perp} \sin \left(\phi_{r}\right), & \boldsymbol{\omega}_{\perp} \cdot \hat{\boldsymbol{e}}_{y}=-\omega_{\perp} \cos \left(\phi_{r}\right)
\end{aligned}
$$


The Jacobian of this transformation is $g$. We get

$$
\begin{aligned}
\left\langle i \mathcal{L}_{+}^{\mathrm{pp}} E_{\mathrm{x} / \mathrm{y}}\right\rangle_{t}= & -\frac{1}{2 \pi^{3 / 2}} a n_{0} g(a)\left(\frac{m}{4 T_{\mathrm{x}}}\right)^{\frac{1}{2}}\left(\frac{m}{4 T_{\mathrm{y}}}\right)^{\frac{1}{2}}\left(\frac{I}{4 T_{\mathrm{rot}}}\right)^{\frac{1}{2}} \int_{\frac{\pi}{2}}^{\frac{3}{2} \pi} d \gamma \int_{0}^{\infty} d g \int_{-\infty}^{\infty} d \omega_{\perp} \int_{0}^{2 \pi} d \phi_{r} g^{2} \cos \gamma \times \\
& \exp \left(-\frac{m}{4 T_{\mathrm{x}}}\left[g^{2} \cos ^{2}\left(\phi_{r}-\gamma\right)-a g \omega_{\perp} \sin \phi_{r} \cos \left(\phi_{r}-\gamma\right)+\frac{a^{2}}{4} \omega_{\perp}^{2} \sin ^{2} \phi_{r}\right]\right) \times \\
& \exp \left(-\frac{m}{4 T_{\mathrm{y}}}\left[g^{2} \sin ^{2}\left(\phi_{r}-\gamma\right)+a g \omega_{\perp} \cos \phi_{r} \sin \left(\phi_{r}-\gamma\right)+\frac{a^{2}}{4} \omega_{\perp}^{2} \cos ^{2} \phi_{r}\right]\right) \times \\
& \exp \left(-\frac{I \omega_{\perp}^{2}}{4 T_{\mathrm{rot}}}\right) \Delta E_{\mathrm{x} / \mathrm{y}} .
\end{aligned}
$$

In this notation the changes of partial energies read

$$
\begin{aligned}
\frac{2}{m} \Delta E_{\mathrm{x}}= & \frac{1-\alpha^{2}}{2} g^{2} \cos ^{2} \gamma \cos ^{2} \phi_{r}+\left[2 \eta(\eta-1) g^{2} \sin ^{2} \gamma+a \eta g \omega_{\perp} \sin \gamma\right] \sin ^{2} \phi_{r} \\
& +\left[(1+\alpha)\left\{(2 \eta-1) g \sin \gamma+\frac{a}{2} \omega_{\perp}\right\} g \cos \gamma-2 \eta g^{2} \sin \gamma \cos \gamma\right] \sin \phi_{r} \cos \phi_{r} \\
\frac{2}{m} \Delta E_{\mathrm{y}}= & \frac{1-\alpha^{2}}{2} g^{2} \cos ^{2} \gamma \sin ^{2} \phi_{r}+\left[2 \eta(\eta-1) g^{2} \sin ^{2} \gamma+a \eta g \omega_{\perp} \sin \gamma\right] \cos ^{2} \phi_{r} \\
& -\left[(1+\alpha)\left\{(2 \eta-1) g \sin \gamma+\frac{a}{2} \omega_{\perp}\right\} g \cos \gamma-2 \eta g^{2} \sin \gamma \cos \gamma\right] \sin \phi_{r} \cos \phi_{r},
\end{aligned}
$$

and

$$
\frac{2}{m} \Delta E_{\mathrm{tr}}=\frac{2}{m}\left(\Delta E_{\mathrm{x}}+\Delta E_{\mathrm{y}}\right)=-\frac{\left(1-\alpha^{2}\right)}{2} g^{2} \cos ^{2} \gamma+2 \eta(\eta-1) g^{2} \sin ^{2} \gamma+a \eta g \omega_{\perp} \sin \gamma
$$

which is in agreement with $\left[\mathrm{HCZ}^{+} 05\right]$. Additionally, we define

$$
\begin{aligned}
\mathcal{A} & :=\frac{m a^{2}}{16}\left[\frac{\sin ^{2} \phi_{r}}{T_{\mathrm{x}}}+\frac{\cos ^{2} \phi_{r}}{T_{\mathrm{y}}}+\frac{q}{T_{\mathrm{rot}}}\right]=\frac{m a^{2}}{16 T_{\mathrm{tr}}} x_{1}^{2}, \\
\mathcal{B} & :=\frac{m a}{8 \mathcal{A}}\left[\frac{\sin \phi_{r} \cos \left(\phi_{r}-\gamma\right)}{T_{\mathrm{x}}}-\frac{\cos \phi_{r} \sin \left(\phi_{r}-\gamma\right)}{T_{\mathrm{y}}}\right]=\frac{2}{a} \frac{x_{2}}{x_{1}^{2}}, \quad \text { and } \\
\mathcal{C} & :=\frac{m}{4}\left[\frac{\cos ^{2}\left(\phi_{r}-\gamma\right)}{T_{\mathrm{x}}}+\frac{\sin ^{2}\left(\phi_{r}-\gamma\right)}{T_{\mathrm{y}}}\right]-\mathcal{A B}^{2}=\frac{m}{4 T_{\mathrm{tr}}}\left(x_{3}^{2}-\frac{x_{2}^{2}}{x_{1}^{2}}\right)
\end{aligned}
$$

where $T_{\mathrm{tr}}:=\left(T_{\mathrm{x}}+T_{\mathrm{y}}\right) / 2, x_{1}^{2}:=\frac{T_{\mathrm{tr}}}{T_{\mathrm{x}}} \sin ^{2} \phi_{r}+\frac{T_{\mathrm{tr}}}{T_{\mathrm{y}}} \cos ^{2} \phi_{r}+\frac{q T_{\mathrm{tr}}}{T_{\mathrm{rot}}}, x_{2}:=\frac{1}{2}\left(\frac{T_{\mathrm{tr}}}{T_{\mathrm{x}}}+\frac{T_{\mathrm{tr}}}{T_{\mathrm{y}}}\right) \sin \gamma+$ $\frac{1}{2}\left(\frac{T_{\text {tr }}}{T_{\mathrm{x}}}-\frac{T_{\text {tr }}}{T_{\mathrm{y}}}\right) \sin \left(2 \phi_{r}-\gamma\right)$, and $x_{3}^{2}:=\frac{T_{\text {tr }}}{T_{\mathrm{x}}} \cos ^{2} \phi_{r}+\frac{T_{\text {tr }}}{T_{\mathrm{y}}} \sin ^{2} \phi_{r}$. Substituting $p_{1}:=\sqrt{\mathcal{C}} g$ for 
$g$ and $p_{2}:=\sqrt{\mathcal{A}}\left(\omega_{\perp}-\mathcal{B} g\right)$ for $\omega_{\perp}$ leads to Gaussian integrals over $p_{1}$ and $p_{2}$, which can be solved easily to yield

$$
\begin{array}{r}
\left\langle i \mathcal{L}_{+}^{\mathrm{pp}} E_{\mathrm{x} / \mathrm{y}}\right\rangle_{t}=-\frac{3}{8 \sqrt{\pi}} a n_{0} g(a)\left(\frac{m}{4 T_{\mathrm{x}}}\right)^{\frac{1}{2}}\left(\frac{m}{4 T_{\mathrm{y}}}\right)^{\frac{1}{2}}\left(\frac{I}{4 T_{\mathrm{rot}}}\right)^{\frac{1}{2}} \times \\
\int_{\frac{\pi}{2}}^{\frac{3}{2} \pi} d \gamma \cos \gamma \int_{0}^{2 \pi} d \phi_{r} \frac{m}{2} \frac{\lambda_{1, x / y}+\mathcal{B} \lambda_{2, x / y}}{\sqrt{\mathcal{A \mathcal { C } ^ { 5 }}}},
\end{array}
$$

where the energy changes, $(2 / m) \Delta E_{\mathrm{x} / \mathrm{y}}=\lambda_{1, x / y} g^{2}+\lambda_{2, x / y} g \omega_{\perp}$ have been expressed using

$$
\begin{aligned}
& \lambda_{1, x}:= \frac{1-\alpha^{2}}{2} \cos ^{2} \gamma \cos ^{2} \phi_{r}+2 \eta(\eta-1) \sin ^{2} \gamma \sin ^{2} \phi_{r} \\
&+[(1+\alpha)(2 \eta-1)-2 \eta] \sin \gamma \cos \gamma \sin \phi_{r} \cos \phi_{r} \\
& \lambda_{2, x}:=a \eta \sin \gamma \sin ^{2} \phi_{r}+(1+\alpha) \frac{a}{2} \cos \gamma \sin \phi_{r} \cos \phi_{r} \\
& \lambda_{1, y}:=\frac{1-\alpha^{2}}{2} \cos ^{2} \gamma \sin ^{2} \phi_{r}+2 \eta(\eta-1) \sin ^{2} \gamma \cos ^{2} \phi_{r} \quad-[(1+\alpha)(2 \eta-1)-2 \eta] \sin \gamma \cos \gamma \sin \phi_{r} \cos \phi_{r} \\
& \lambda_{2, y}:=a \eta \sin \gamma \cos ^{2} \phi_{r}-(1+\alpha) \frac{a}{2} \cos \gamma \sin \phi_{r} \cos \phi_{r},
\end{aligned}
$$

\section{Special Case 1: PP Contribution $\left\langle i \mathcal{L}_{+}^{\mathrm{pp}} E_{\mathrm{tr}}\right\rangle_{t}$ Assuming an Isotropic Translational Temperature $T_{\mathrm{tr}}$}

Assuming an isotropic temperature $T_{\mathrm{x}}(t)=T_{\mathrm{y}}(t)=T_{\mathrm{tr}}(t)$ for all times, Eq. (C.26) simplifies significantly: The coefficients $\mathcal{A}, \mathcal{B}$, and $\mathcal{C}$, Eq. (C.25), simplify to $\widetilde{\mathcal{A}}=\left.\mathcal{A}\right|_{T_{\mathrm{x}}=T_{\mathrm{y}}}=\frac{m a^{2}}{16 T_{\mathrm{tr}}} \widetilde{x}_{1}^{2}=$ $\frac{m a^{2}}{16 T_{\text {tr }}} \frac{x^{2}}{x^{2}-1}$, where $\widetilde{x}_{1}^{2}=\left.x_{1}^{2}\right|_{T_{\mathrm{x}}=T_{\mathrm{y}}}=1+q T_{\text {tr }} / T_{\text {rot }}$ and $x^{2}=1+T_{\text {rot }} /\left[q T_{\text {tr }}\right], \widetilde{\mathcal{B}}=\left.\mathcal{B}\right|_{T_{\mathrm{x}}=T_{\mathrm{y}}}=$ $\frac{2}{a} \frac{\sin \gamma}{\widetilde{x}_{1}^{2}}=\frac{2}{a} \frac{x^{2}-1}{x^{2}} \sin \gamma$, and $\widetilde{\mathcal{C}}=\left.\mathcal{C}\right|_{T_{\mathrm{x}}=T_{\mathrm{y}}}=\frac{m}{4 T_{\mathrm{tr}}}\left(1-\frac{\sin ^{2} \gamma}{\widetilde{x}_{1}^{2}}\right)=\frac{m}{4 T_{\mathrm{tr}} x^{2}}\left(1-\left(x^{2}-1\right) \cos ^{2} \gamma\right)$, i.e. all three become independent of $\phi_{r}$ while still being functions of $T_{\mathrm{x}}$ and $T_{\mathrm{y}}$. Additionally, $\widetilde{\mathcal{B}}$ and $\widetilde{\mathcal{C}}$ depend also on $\gamma$. Then the integration over $\phi_{r}$ in Eq. (C.26) can be performed to yield a factor $\pi$. We get

$$
\left\langle i \mathcal{L}_{+}^{\mathrm{pp}} E_{\mathrm{x} / \mathrm{y}}\right\rangle_{t}=-\frac{3}{8} \sqrt{\pi} a n_{0} g(a)\left(\frac{m}{4 T_{\mathrm{tr}}}\right)\left(\frac{I}{4 T_{\mathrm{rot}}}\right)^{\frac{1}{2}} \int_{\frac{\pi}{2}}^{\frac{3}{2} \pi} d \gamma \cos \gamma \frac{m}{2} \frac{\widetilde{\lambda}_{1}+\widetilde{\mathcal{B}} \widetilde{\lambda}_{2}}{\sqrt{\widetilde{\mathcal{A}} \widetilde{\mathcal{C}}^{5}}},
$$

where $\widetilde{\lambda}_{1}=\frac{1-\alpha^{2}}{2} \cos ^{2} \gamma+2 \eta(\eta-1) \sin ^{2} \gamma$ and $\widetilde{\lambda}_{2}=a \eta \sin \gamma$. Eq. (C.28) can be simplified to yield

$$
\left\langle i \mathcal{L}_{+}^{\mathrm{pp}} E_{\mathrm{x} / \mathrm{y}}\right\rangle_{t}=-\frac{3}{4} \sqrt{\frac{\pi}{m}} a n_{0} g(a) T_{\mathrm{tr}}^{\frac{3}{2}} x^{4} \int_{\frac{\pi}{2}}^{\frac{3}{2} \pi} d \gamma \frac{\left(\frac{1-\alpha^{2}}{2} \cos ^{3} \gamma+2 \eta\left(\eta-\frac{1}{x^{2}}\right) \sin ^{2} \gamma \cos \gamma\right)}{\left(1-\left(x^{2}-1\right) \cos ^{2} \gamma\right)^{5 / 2}}
$$


Note that $\left\langle i \mathcal{L}_{+}^{\mathrm{pp}} E_{\mathrm{x}}\right\rangle_{t}=\left\langle i \mathcal{L}_{+}^{\mathrm{pp}} E_{\mathrm{y}}\right\rangle_{t}=\frac{1}{2}\left\langle i \mathcal{L}_{+}^{\mathrm{pp}} E_{\mathrm{x} / \mathrm{y}}\right\rangle_{t}$. We will go on evaluating $\left\langle i \mathcal{L}_{+}^{\mathrm{pp}} E_{\mathrm{x} / \mathrm{y}}\right\rangle_{t}$.

Up to this point we have not specified, whether we are going to use constant coefficients of restitution or Coulomb friction. All this is hidden in $\eta$ which is either a constant or a function of $\gamma$. We are interested in the Coulomb friction case so we use $\eta=\eta(\gamma)$. We introduce the notation $\eta=\frac{1+\alpha}{2} \mu \min \left\{\left|\cot \gamma_{0}\right|,|\cot \gamma|\right\} \equiv \min \left\{\eta_{0}, \frac{1+\alpha}{2} \mu|\cot \gamma|\right\}$ with $\eta_{0}:=\frac{q}{1+q} \frac{1+\beta_{0}}{2}$ and obtain

$$
\begin{aligned}
\left\langle i \mathcal{L}_{+}^{\mathrm{pp}} E_{\mathrm{tr}}\right\rangle_{t}=- & \frac{3}{2} \sqrt{\frac{\pi}{m}} a n_{0} g(a) T_{\mathrm{tr}}^{\frac{3}{2}} x^{4}\left\{\frac{2}{3} \frac{1-\alpha^{2}}{x^{4}}\right. \\
& +\int_{\frac{\pi}{2}}^{\gamma_{0}} d \gamma \frac{\cos \gamma}{\left(1+\left(x^{2}-1\right) \cos ^{2} \gamma\right)^{5 / 2}}\left(2 \mu \frac{1+\alpha}{x^{2}} \sin \gamma \cos \gamma+\mu^{2}(1+\alpha)^{2} \cos ^{2} \gamma\right) \\
& \left.+4 \eta_{0}\left(\eta_{0}-\frac{1}{x^{2}}\right) \int_{\gamma_{0}}^{\pi} d \gamma \frac{\cos \gamma \sin ^{2} \gamma}{\left(1+\left(x^{2}-1\right) \cos ^{2} \gamma\right)^{5 / 2}}\right\}
\end{aligned}
$$

After performing the last integration the result can be written in the form

$$
\begin{aligned}
\frac{d}{d t} T_{\mathrm{tr}}= & H_{\mathrm{dr}}-G T_{\mathrm{tr}}^{3 / 2}\left\{A_{r}\right. \\
& +\frac{\eta_{0}}{2} \frac{1-\eta_{0} x^{2}}{\left(1+x^{2} \cot ^{2} \gamma_{0}\right)^{3 / 2}}+\frac{\eta_{0}}{2} \frac{x^{2} \cot ^{2} \gamma_{0}}{\left(1+x^{2} \cot ^{2} \gamma_{0}\right)^{3 / 2}} \\
& \left.-\eta_{0}^{2} \tan ^{2} \gamma_{0}\left(1-\frac{1+\frac{3}{2} x^{2} \cot ^{2} \gamma_{0}}{\left(1+x^{2} \cot ^{2} \gamma_{0}\right)^{3 / 2}}\right)\right\}
\end{aligned}
$$

where $G=4 \sqrt{\frac{\pi}{m}} a n_{0} g(a)$, which is the same as Eq. (10.5), and $A_{r}=\frac{1-\alpha^{2}}{4}$.

\section{Special Case 2: PP Contribution $\left\langle i \mathcal{L}_{+}^{\mathrm{pp}} E_{\mathrm{x} / \mathrm{y}}\right\rangle_{t}$ for Smooth Particles}

For smooth disks or spheres, $\eta=0$. Going back to Eq. (C.26) the simplified lambdas read

$$
\begin{aligned}
\lambda_{1, x} & :=\frac{1-\alpha^{2}}{2} \cos ^{2} \gamma \cos ^{2} \phi_{r}-(1+\alpha) \sin \gamma \cos \gamma \sin \phi_{r} \cos \phi_{r} \\
\lambda_{2, x} & :=(1+\alpha) \frac{a}{2} \cos \gamma \sin \phi_{r} \cos \phi_{r} \\
\lambda_{1, y} & :=\frac{1-\alpha^{2}}{2} \cos ^{2} \gamma \sin ^{2} \phi_{r}+(1+\alpha) \sin \gamma \cos \gamma \sin \phi_{r} \cos \phi_{r} \\
\lambda_{2, y} & :=-(1+\alpha) \frac{a}{2} \cos \gamma \sin \phi_{r} \cos \phi_{r} .
\end{aligned}
$$


They have to be inserted into Eq. (C.26). We get

$$
\begin{aligned}
& \left\langle i \mathcal{L}_{+}^{\mathrm{pp}} E_{\mathrm{x} / \mathrm{y}}\right\rangle_{t}=-3 \sqrt{\frac{\pi}{m}} a n_{0} g(a)\left(\frac{T_{\mathrm{tr}}}{T_{\mathrm{x}}}\right)^{\frac{1}{2}}\left(\frac{T_{\mathrm{tr}}}{T_{\mathrm{y}}}\right)^{\frac{1}{2}}\left(\frac{q T_{\mathrm{tr}}}{T_{\mathrm{rot}}}\right)^{\frac{1}{2}} T_{\mathrm{tr}}^{\frac{3}{2}} \times \\
& \int_{\frac{\pi}{2}}^{\frac{3}{2} \pi} d \gamma \cos \gamma \int_{0}^{2 \pi} d \phi_{r} \frac{\frac{1-\alpha^{2}}{2} \cos ^{2} \gamma=(1+\alpha)\left[\frac{x_{2}}{x_{1}^{2}}-\sin \gamma\right] \cos \gamma \sin \phi_{r} \cos \phi_{r}}{\sqrt{x_{1}^{2}\left(x_{3}^{2}-\frac{x_{2}^{2}}{x_{1}^{2}}\right)^{5}}}
\end{aligned}
$$

where $x_{1}^{2}:=\frac{T_{\mathrm{tr}}}{T_{\mathrm{x}}} \sin ^{2} \phi_{r}+\frac{T_{\mathrm{tr}}}{T_{\mathrm{y}}} \cos ^{2} \phi_{r}+\frac{q T_{\mathrm{tr}}}{T_{\mathrm{rot}}}, x_{2}:=\frac{1}{2}\left(\frac{T_{\mathrm{tr}}}{T_{\mathrm{x}}}+\frac{T_{\mathrm{tr}}}{T_{\mathrm{y}}}\right) \sin \gamma+\frac{1}{2}\left(\frac{T_{\mathrm{tr}}}{T_{\mathrm{x}}}-\right.$ $\left.\frac{T_{\mathrm{tr}}}{T_{\mathrm{y}}}\right) \sin \left(2 \phi_{r}-\gamma\right)$, and $x_{3}^{2}:=\frac{T_{\mathrm{tr}}}{T_{\mathrm{x}}} \cos ^{2} \phi_{r}+\frac{T_{\mathrm{tr}}}{T_{\mathrm{y}}} \sin ^{2} \phi_{r}$ as defined in the lines below Eq. (C.25).

An easier approach for smooth particles is to perform the integration over $\phi_{r}$ before doing anything else. Especially, we want to avoid substitution (C.17). Therefore, we go back to Eq. (C.14) with $\Delta E_{\mathrm{x} / \mathrm{y}}$ as given in Eq. (C.15). Since $\eta \equiv 0$ for smooth particles, $\Delta E_{\mathrm{x} / \mathrm{y}}$ simplifies to

$$
\frac{2}{m} \Delta E_{\mathrm{x} / \mathrm{y}}=(1+\alpha)^{2}(\hat{\boldsymbol{r}} \cdot \boldsymbol{v})^{2}\left(\hat{\boldsymbol{r}} \cdot \hat{\boldsymbol{e}}_{x / y}\right)^{2}-2(1+\alpha)(\hat{\boldsymbol{r}} \cdot \boldsymbol{v})\left(\hat{\boldsymbol{r}} \cdot \hat{\boldsymbol{e}}_{x / y}\right)\left(\boldsymbol{v} \cdot \hat{\boldsymbol{e}}_{x / y}\right)
$$

such that we can do the integration over $d \phi_{r}$. Therefore, we integrate

$$
\begin{aligned}
\int_{0}^{2 \pi} d \phi_{r}\left(\hat{\boldsymbol{r}} \cdot \boldsymbol{v} \Theta(-\hat{\boldsymbol{r}} \cdot \hat{\boldsymbol{v}}) \frac{2}{m} \Delta E_{\mathrm{x}}\right) & =\int_{0}^{2 \pi} d \phi_{r}\left(v \cos \left(\phi_{r}-\phi_{v}\right) \Theta\left(-\cos \left(\phi_{r}-\phi_{v}\right)\right) \frac{2}{m} \Delta E_{\mathrm{x}}\right) \\
& =\frac{4}{3}(1+\alpha)\left(\frac{-1-\alpha}{5}\left(1+3 \cos ^{2} \phi_{v}\right)+2 \cos ^{2} \phi_{v}\right) v^{3} \\
& =\frac{4}{3}\left(\mathcal{F}_{x}+\mathcal{F}_{0} \cos ^{2} \phi_{v}\right) v^{3}=: v^{3} \frac{2}{m} \Delta \widetilde{E}_{\mathrm{x}}
\end{aligned}
$$

and

$$
\begin{aligned}
\int_{0}^{2 \pi} d \phi_{r}\left(\hat{\boldsymbol{r}} \cdot \boldsymbol{v} \Theta(-\hat{\boldsymbol{r}} \cdot \hat{\boldsymbol{v}}) \frac{2}{m} \Delta E_{\mathrm{y}}\right) & =\int_{0}^{2 \pi} d \phi_{r}\left(v \cos \left(\phi_{r}-\phi_{v}\right) \Theta\left(-\cos \left(\phi_{r}-\phi_{v}\right)\right) \frac{2}{m} \Delta E_{\mathrm{y}}\right) \\
& =\frac{4}{3}(1+\alpha)\left(\frac{-1-\alpha}{5}\left(4-3 \cos ^{2} \phi_{v}\right)+2 \sin ^{2} \phi_{v}\right) v^{3} \\
& =\frac{4}{3}\left(\mathcal{F}_{y}-\mathcal{F}_{0} \cos ^{2} \phi_{v}\right) v^{3}=: v^{3} \frac{2}{m} \Delta \widetilde{E}_{\mathrm{y}}
\end{aligned}
$$

with $\mathcal{F}_{x}:=(1+\alpha) \frac{-1-\alpha}{5}=-\frac{1}{5}(1+\alpha)^{2}, \mathcal{F}_{y}:=(1+\alpha) \frac{6-4 \alpha}{5}=\left(1-\alpha^{2}\right)+\frac{1}{5}(1+\alpha)^{2}=$ $\left(1-\alpha^{2}\right)-\mathcal{F}_{x}$ and $\mathcal{F}_{0}:=(1+\alpha) \frac{7-3 \alpha}{5}=\left(1-\alpha^{2}\right)+\frac{2}{5}(1+\alpha)^{2}$.

[Consistency check: $v^{3} \frac{2}{m} \Delta \widetilde{E}_{\mathrm{tr}}=v^{3} \frac{2}{m} \Delta \widetilde{E}_{\mathrm{x}}+v^{3} \frac{2}{m} \Delta \widetilde{E}_{\mathrm{y}}=\frac{4}{3}\left(1-\alpha^{2}\right) v^{3}$. This is a function of $v=|\boldsymbol{v}|$ only. Thus, when calculating the total change of energy the integration over $d \phi_{v}$, 
$\int_{0}^{2 \pi} d \phi_{v} v^{3} \frac{2}{m} \Delta \widetilde{E}_{\mathrm{tr}}=2 \pi \frac{4}{3}\left(1-\alpha^{2}\right) v^{3}$, simply yields a factor of $2 \pi$.] Inserting Eqs. (C.35) or (C.36) into Eq. (C.14) and integrating over $d \omega$ we get

$$
\begin{aligned}
\left\langle i \mathcal{L}_{+}^{\mathrm{pp}} E_{\mathrm{x} / \mathrm{y}}\right\rangle_{t}=- & \frac{1}{\sqrt{2}} a n_{0} g(a)\left(\frac{m}{2 \pi T_{\mathrm{x}}}\right)^{\frac{1}{2}}\left(\frac{m}{2 \pi T_{\mathrm{y}}}\right)^{\frac{1}{2}} \int_{0}^{2 \pi} d \phi_{v} \int_{0}^{\infty} d v v \times \\
& \quad \exp \left(-\frac{m}{2}\left[\frac{v^{2} \cos ^{2} \phi_{v}}{T_{\mathrm{x}}}+\frac{v^{2} \sin ^{2} \phi_{v}}{T_{\mathrm{y}}}\right]\right) v^{3} \Delta \widetilde{E}_{\mathrm{x} / \mathrm{y}}
\end{aligned}
$$

where

$$
v^{3} \Delta \widetilde{E}_{\mathrm{x} / \mathrm{y}}=\frac{2}{3} m v^{3}\left(\mathcal{F}_{x / y} \pm \mathcal{F}_{0} \cos ^{2} \phi_{v}\right) .
$$

The integration over $v$ is a Gaussian type integral and we get

$$
\left\langle i \mathcal{L}_{+}^{\mathrm{pp}} E_{\mathrm{x} / \mathrm{y}}\right\rangle_{t}=-\frac{a n_{0} g(a)}{2 \pi} \sqrt{\frac{\pi}{m}} \frac{1}{\sqrt{T_{\mathrm{x}} T_{\mathrm{y}}}} \int_{0}^{2 \pi} d \phi_{v} \frac{\mathcal{F}_{x / y} \pm \mathcal{F}_{0} \cos ^{2} \phi_{v}}{\left(\frac{\cos ^{2}\left(\phi_{v}\right)}{T_{\mathrm{x}}}+\frac{\sin ^{2}\left(\phi_{v}\right)}{T_{\mathrm{y}}}\right)^{\frac{5}{2}}}
$$

The remaining integral is an elliptic integral, more precisely number 2.581,3 in Gradshteyn [GR65], page 157 with $a:=T_{\mathrm{x}}^{-1}, b:=T_{\mathrm{y}}^{-1}$ and $k^{2}:=\max \left(1-\frac{a}{b}, 1-\frac{b}{a}\right)$, where we assume without loss of generality $b<a$. Defining $k_{b a}^{2}:=1-\frac{b}{a}$ we get:

$$
\begin{aligned}
\int_{0}^{2 \pi} d \phi_{v} & \frac{\mathcal{F}_{x / y} \pm \mathcal{F}_{0} \cos ^{2} \phi_{v}}{\left(\frac{\cos ^{2}\left(\phi_{v}\right)}{T_{\mathrm{x}}}+\frac{\sin ^{2}\left(\phi_{v}\right)}{T_{\mathrm{y}}}\right)^{\frac{5}{2}}}=\int_{0}^{2 \pi} d \phi_{v} \frac{\mathcal{F}_{x / y} \pm \mathcal{F}_{0} \cos ^{2} \phi_{v}}{\sqrt{a \cos ^{2}\left(\phi_{v}\right)+b \sin ^{2}\left(\phi_{v}\right)}} \\
= & \frac{1}{\sqrt{a}^{5}} \int_{0}^{2 \pi} d \phi_{v} \frac{\mathcal{F}_{x / y} \pm \mathcal{F}_{0} \cos ^{2} \phi_{v}}{\sqrt{1-k_{b a}^{2} \sin ^{2}\left(\phi_{v}\right)}}=: \frac{\mathcal{F}_{x / y}}{\sqrt{a}^{5}} f_{1}\left(k_{b a}\right) \pm \frac{\mathcal{F}_{0}}{\sqrt{a}^{5}} f_{c}\left(k_{b a}\right)
\end{aligned}
$$

where

$$
f_{1}(k)=\frac{4}{3} \frac{2 k\left(2-k^{2}\right)}{\left(1-k^{2}\right)^{2}} \operatorname{EllipticE}(k, 1 / k)+\frac{4}{3} \frac{3-2 k^{2}}{k\left(1-k^{2}\right)} \operatorname{EllipticF}(k, 1 / k)
$$

and

$$
f_{c}(k)=-\frac{4}{3} \frac{1-2 k^{2}}{k\left(1-k^{2}\right)} \text { EllipticE }(k, 1 / k)+\frac{8}{3 k} \operatorname{EllipticF}(k, 1 / k)
$$

with

$$
\begin{aligned}
& \operatorname{EllipticE}(k, 1 / k)=\int_{0}^{k} \sqrt{\frac{1-t^{2} / k^{2}}{1-t^{2}}} d t \\
& \operatorname{EllipticF}(k, 1 / k)=\int_{0}^{k} \frac{1}{\sqrt{\left(1-t^{2} / k^{2}\right)\left(1-t^{2}\right)}} d t
\end{aligned}
$$


For $a<b$ and $k_{a b}^{2}:=1-\frac{a}{b}$ we get:

$$
\begin{aligned}
& \int_{0}^{2 \pi} d \phi_{v} \frac{\mathcal{F}_{x / y} \pm \mathcal{F}_{0} \cos ^{2} \phi_{v}}{\sqrt{a \cos ^{2}\left(\phi_{v}\right)+b \sin ^{2}\left(\phi_{v}\right)}}=\int_{-\frac{\pi}{2}}^{\frac{3}{2} \pi} d \phi_{v} \frac{\mathcal{F}_{x / y} \pm \mathcal{F}_{0} \cos ^{2} \phi_{v}}{\sqrt{a \cos ^{2}\left(\phi_{v}\right)+b \sin ^{2}\left(\phi_{v}\right)}}{ }^{5} \\
& =\int_{0}^{2 \pi} d \phi_{v} \frac{\mathcal{F}_{x / y} \pm \mathcal{F}_{0} \cos ^{2}\left(\phi_{v}-\frac{\pi}{2}\right)}{\sqrt{a \cos ^{2}\left(\phi_{v}-\frac{\pi}{2}\right)+b \sin ^{2}\left(\phi_{v}-\frac{\pi}{2}\right)}}=\int_{0}^{2 \pi} d \phi_{v} \frac{\mathcal{F}_{x / y} \pm \mathcal{F}_{0} \sin ^{2} \phi_{v}}{\sqrt{a \sin ^{2}\left(\phi_{v}\right)+b \cos ^{2}\left(\phi_{v}\right)}} \\
& =\frac{1}{\sqrt{b}^{5}} \int_{0}^{2 \pi} d \phi_{v} \frac{\mathcal{F}_{x / y} \pm \mathcal{F}_{0} \sin ^{2} \phi_{v}}{\sqrt{1-k_{a b}^{2} \sin ^{2}\left(\phi_{v}\right)}}=: \frac{\mathcal{F}_{x / y}}{\sqrt{b}^{5}} f_{1}\left(k_{a b}\right) \pm \frac{\mathcal{F}_{0}}{\sqrt{b}^{5} f_{s}\left(k_{a b}\right)}
\end{aligned}
$$

where

$$
f_{s}(k)=\frac{4}{3} \frac{1+k^{2}}{k\left(1-k^{2}\right)^{2}} \operatorname{EllipticE}(k, 1 / k)+\frac{4}{3} \frac{1}{k\left(1-k^{2}\right)} \operatorname{EllipticF}(k, 1 / k)
$$

Therefore we get

$$
\begin{aligned}
\left\langle i \mathcal{L}_{+}^{\mathrm{pp}} E_{\mathrm{x} / \mathrm{y}}\right\rangle_{t} & =-\frac{a n_{0} g(a)}{2 \pi} \sqrt{\frac{\pi}{m}} T_{\mathrm{tr}}{ }^{\frac{3}{2}} \times \\
& \left\{\Theta\left(T_{\mathrm{x}}-T_{\mathrm{y}}\right)\left(\frac{T_{\mathrm{y}}}{T_{\mathrm{tr}}}\right)^{\frac{3}{2}} \sqrt{\frac{T_{\mathrm{y}}}{T_{\mathrm{x}}}}\left(\mathcal{F}_{x / y} f_{1}\left(k_{x y}\right) \pm \mathcal{F}_{0} f_{s}\left(k_{x y}\right)\right)\right. \\
& \left.+\Theta\left(T_{\mathrm{y}}-T_{\mathrm{x}}\right)\left(\frac{T_{\mathrm{x}}}{T_{\mathrm{tr}}}\right)^{\frac{3}{2}} \sqrt{\frac{T_{\mathrm{x}}}{T_{\mathrm{y}}}}\left(\mathcal{F}_{x / y} f_{1}\left(k_{y x}\right) \pm \mathcal{F}_{0} f_{c}\left(k_{y x}\right)\right)\right\}
\end{aligned}
$$

This can also be expressed without $f_{s}(k)$ by noting that

$$
\begin{aligned}
\int_{0}^{2 \pi} d \phi_{v} \frac{\mathcal{F}_{x / y} \pm \mathcal{F}_{0} \cos ^{2} \phi_{v}}{\sqrt{a \cos ^{2}\left(\phi_{v}\right)+b \sin ^{2}\left(\phi_{v}\right)}}=\frac{1}{\sqrt{b}^{5}} \int_{0}^{2 \pi} d \phi_{v} \frac{\mathcal{F}_{x / y} \pm \mathcal{F}_{0} \sin ^{2} \phi_{v}}{\sqrt{1-k_{a b}^{2} \sin ^{2}\left(\phi_{v}\right)}} \\
=\frac{1}{\sqrt{b}^{5}} \int_{0}^{2 \pi} d \phi_{v} \frac{\left(\mathcal{F}_{x / y} \pm \mathcal{F}_{0}\right) \mp \mathcal{F}_{0} \cos ^{2} \phi_{v}}{\sqrt{1-k_{a b}^{2} \sin ^{2}\left(\phi_{v}\right)}}=: \frac{\mathcal{F}_{x / y} \pm \mathcal{F}_{0}}{\sqrt{b}^{5}} f_{1}\left(k_{a b}\right) \mp \frac{\mathcal{F}_{0}}{\sqrt{b}^{5}} f_{c}\left(k_{a b}\right)
\end{aligned}
$$

which yields

$$
\begin{aligned}
&\left\langle i \mathcal{L}_{+}^{\mathrm{pp}} E_{\mathrm{x} / \mathrm{y}}\right\rangle_{t}=-\frac{a n_{0} g(a)}{2 \pi} \sqrt{\frac{\pi}{m}} T_{\mathrm{tr}}^{\frac{3}{2}} \times \\
&\left\{\Theta\left(T_{\mathrm{x}}-T_{\mathrm{y}}\right)\left(\frac{T_{\mathrm{y}}}{T_{\mathrm{tr}}}\right)^{\frac{3}{2}} \sqrt{\frac{T_{\mathrm{y}}}{T_{\mathrm{x}}}}\left(\left(\mathcal{F}_{x / y} \pm \mathcal{F}_{0}\right) f_{1}\left(k_{x y}\right) \mp \mathcal{F}_{0} f_{c}\left(k_{x y}\right)\right)\right. \\
&\left.+\Theta\left(T_{\mathrm{y}}-T_{\mathrm{x}}\right)\left(\frac{T_{\mathrm{x}}}{T_{\mathrm{tr}}}\right)^{\frac{3}{2}} \sqrt{\frac{T_{\mathrm{x}}}{T_{\mathrm{y}}}}\left(\mathcal{F}_{x / y} f_{1}\left(k_{y x}\right) \pm \mathcal{F}_{0} f_{c}\left(k_{y x}\right)\right)\right\}
\end{aligned}
$$


where $T_{\mathrm{tr}}=\frac{1}{2}\left(T_{\mathrm{x}}+T_{\mathrm{y}}\right), k_{x y}:=\sqrt{1-\frac{T_{\mathrm{y}}}{T_{\mathrm{x}}}}, k_{y x}:=\sqrt{1-\frac{T_{\mathrm{x}}}{T_{\mathrm{y}}}}, \mathcal{F}_{x}:=-\frac{1}{5}(1+\alpha)^{2}, \mathcal{F}_{y}:=$ $\left(1-\alpha^{2}\right)+\frac{1}{5}(1+\alpha)^{2}=\left(1-\alpha^{2}\right)-\mathcal{F}_{x}$ and $\mathcal{F}_{0}:=\left(1-\alpha^{2}\right)+\frac{2}{5}(1+\alpha)^{2}$. Here again $\Theta($. is the Heaviside step function such that $\Theta(0):=\frac{1}{2}$. Noting that $\mathcal{F}_{x / y} \pm \mathcal{F}_{0}=\mathcal{F}_{y / x}$ we get the final result

$$
\begin{aligned}
\left\langle i \mathcal{L}_{+}^{\mathrm{pp}} E_{\mathrm{x} / \mathrm{y}}\right\rangle_{t} & =-\frac{a n_{0} g(a)}{2 \pi} \sqrt{\frac{\pi}{m}} T_{\mathrm{tr}}^{\frac{3}{2}} \times \\
& \left\{\Theta\left(T_{\mathrm{x}}-T_{\mathrm{y}}\right)\left(\frac{T_{\mathrm{y}}}{T_{\mathrm{tr}}}\right)^{\frac{3}{2}} \sqrt{\frac{T_{\mathrm{y}}}{T_{\mathrm{x}}}}\left(\mathcal{F}_{y / x} f_{1}\left(k_{x y}\right) \mp \mathcal{F}_{0} f_{c}\left(k_{x y}\right)\right)\right. \\
+ & \left.\Theta\left(T_{\mathrm{y}}-T_{\mathrm{x}}\right)\left(\frac{T_{\mathrm{x}}}{T_{\mathrm{tr}}}\right)^{\frac{3}{2}} \sqrt{\frac{T_{\mathrm{x}}}{T_{\mathrm{y}}}}\left(\mathcal{F}_{x / y} f_{1}\left(k_{y x}\right) \pm \mathcal{F}_{0} f_{c}\left(k_{y x}\right)\right)\right\}
\end{aligned}
$$

As a consistency check we validate that indeed for $T_{x}=T_{y}$ we get $\left\langle i \mathcal{L}_{+}^{\mathrm{pp}} E_{\mathrm{x}}\right\rangle_{t}=\left\langle i \mathcal{L}_{+}^{\mathrm{pp}} E_{\mathrm{y}}\right\rangle_{t}=$ $\frac{1}{2}\left\langle i \mathcal{L}_{+}^{\mathrm{pp}} E_{\mathrm{tr}}\right\rangle_{t}$ because $\Theta(0) \mathcal{F}_{y / x}+\Theta(0) \mathcal{F}_{x / y}=\frac{1}{2}\left(\mathcal{F}_{x}+\mathcal{F}_{y}\right)=\frac{1}{2}\left(1-\alpha^{2}\right)$ and for $T_{x}=T_{y}$ we find $k_{x y}=k_{y x}=0$ and $f_{1}(0)=2 \pi$. That means: If a system without walls etc. ever reaches $T_{x}=T_{y}$ then this will prevail forever. This is expected for the stationary state without walls.

For $T_{x}=0$ and $T_{y}=0$, of course we get $\left\langle i \mathcal{L}_{+}^{\mathrm{pp}} E_{\mathrm{x}}\right\rangle_{t}=\left\langle i \mathcal{L}_{+}^{\mathrm{pp}} E_{\mathrm{y}}\right\rangle_{t}=0$.

If at some instance of time $T_{x}=0$ while $T_{y} \neq 0$, the integrations simplify because we do not have to integrate over $v_{x}$ any more but instead $v_{x} \equiv 0$. In this case we use Cartesian instead of polar coordinates. When $v_{x}=0$ we get (starting from Eqs. (C.37) and (C.38))

$$
\left\langle i \mathcal{L}_{+}^{\mathrm{pp}} E_{\mathrm{x} / \mathrm{y}}\right\rangle_{t}=-\frac{1}{\sqrt{2}} a n_{0} g(a)\left(\frac{m}{2 \pi T_{\mathrm{y}}}\right)^{\frac{1}{2}} \int_{-\infty}^{\infty} d v_{y} \exp \left(-\frac{m}{2} \frac{\left|v_{y}\right|^{2}}{T_{\mathrm{y}}}\right)\left|v_{y}\right|^{3}\left[\Delta \widetilde{E}_{\mathrm{x} / \mathrm{y}}\right]_{v_{x}=0}
$$

with

$$
\left|v_{y}\right|^{3}\left[\Delta \widetilde{E}_{\mathrm{x} / \mathrm{y}}\right]_{v_{x}=0}=\frac{2}{3} m\left|v_{y}\right|^{3} \mathcal{F}_{x / y}
$$

Thus we get

$$
\begin{aligned}
& T_{x}=0 \quad \Rightarrow \quad\left\langle i \mathcal{L}_{+}^{\mathrm{pp}} E_{\mathrm{x} / \mathrm{y}}\right\rangle_{t}=-\frac{4}{3} \frac{1}{\sqrt{m \pi}} a n_{0} g(a) \mathcal{F}_{x / y} T_{\mathrm{y}}^{\frac{3}{2}} \\
& T_{y}=0 \quad \Rightarrow \quad\left\langle i \mathcal{L}_{+}^{\mathrm{pp}} E_{\mathrm{x} / \mathrm{y}}\right\rangle_{t}=-\frac{4}{3} \frac{1}{\sqrt{m \pi}} a n_{0} g(a) \mathcal{F}_{y / x} T_{\mathrm{x}}^{\frac{3}{2}}
\end{aligned}
$$

in agreement with Eq. (C.49) when we remember that $\lim _{T_{x / y} \rightarrow 0}\left(T_{x / y} / T_{y / x}\right)^{2} f_{1}\left(k_{y x / x y}\right)=\frac{8}{3}$ and $\lim _{T_{x / y} \rightarrow 0}\left(T_{x / y} / T_{y / x}\right)^{2} f_{c}\left(k_{y x / x y}\right)=0$.

It can be easily seen that for $T_{x}=0$ we get $\left\langle i \mathcal{L}_{+}^{\mathrm{pp}} E_{\mathrm{x}}\right\rangle_{t}>0$, while $\left\langle i \mathcal{L}_{+}^{\mathrm{pp}} E_{\mathrm{y}}\right\rangle_{t}<0$ - independently of $\alpha$. 


\section{C.2.2. PP Contribution $\left\langle i \mathcal{L}_{+}^{\mathrm{pp}} E_{\mathrm{rot}}\right\rangle_{t}$ to the Change of Rotational Energy}

Similarly to $\left\langle i \mathcal{L}_{+}^{\mathrm{pp}} E_{\mathrm{x} / \mathrm{y}}\right\rangle_{t}$ in section C.2.1 we can calculate $\left\langle i \mathcal{L}_{+}^{\mathrm{pp}} E_{\mathrm{rot}}\right\rangle_{t}$. The calculation is very similar until Eq. (C.26) as we will see. Obviously, the calculation is identical to the one for $\left\langle i \mathcal{L}_{+}^{\mathrm{pp}} E_{\mathrm{x} / \mathrm{y}}\right\rangle_{t}$ until Eq. (C.14). Instead of $\Delta E_{\mathrm{x} / \mathrm{y}}$ we need

$$
\begin{aligned}
\frac{2}{I} \Delta E_{\mathrm{rot}} & :=\left(b_{+}^{12}-1\right)\left(\left|\boldsymbol{\omega}_{1}\right|^{2}+\left|\boldsymbol{\omega}_{2}\right|^{2}\right)=\left(\left|\boldsymbol{\omega}_{1}^{\prime}\right|^{2}+\left|\boldsymbol{\omega}_{2}^{\prime}\right|^{2}\right)-\left(\left|\boldsymbol{\omega}_{1}\right|^{2}+\left|\boldsymbol{\omega}_{2}\right|^{2}\right) \\
& \stackrel{(2.4,2.5)}{=} \frac{16 \eta^{2}}{a^{2} q^{2}}|\hat{\boldsymbol{r}} \times \boldsymbol{v}|^{2}+4 \frac{\eta}{q}\left(\frac{\eta}{q}-1\right)\left(|\boldsymbol{\omega}|^{2}-(\hat{\boldsymbol{r}} \cdot \boldsymbol{\omega})^{2}\right)-\frac{8 \eta}{a q}\left(2 \frac{\eta}{q}-1\right)(\hat{\boldsymbol{r}} \times \boldsymbol{v}) \cdot \boldsymbol{\omega} .
\end{aligned}
$$

Using the notation introduced in Eqs. (C.13), (C.17), and (C.21) this can be written as

$$
\frac{2}{m} \Delta E_{\mathrm{rot}}=\frac{2 \eta^{2}}{q}\left(|\boldsymbol{g}|^{2}-(\hat{\boldsymbol{r}} \cdot \boldsymbol{g})^{2}\right)-a \eta \boldsymbol{g} \cdot \boldsymbol{\omega}_{\perp}=\frac{2 \eta^{2}}{q} g^{2} \sin ^{2} \gamma-a \eta g \omega_{\perp} \sin \gamma
$$

Eq. (C.54) reveals that, indeed, the calculation for $\left\langle i \mathcal{L}_{+}^{\mathrm{pp}} E_{\text {rot }}\right\rangle_{t}$ stays identical to the one for $\left\langle i \mathcal{L}_{+}^{\mathrm{pp}} E_{\mathrm{x} / \mathrm{y}}\right\rangle_{t}$ shown in Sec. C.2.1 until Eq. (C.26) where we have to use

$$
\lambda_{1, r o t}:=\frac{2 \eta^{2}}{q} \sin ^{2} \gamma \quad \text { and } \quad \lambda_{2, r o t}:=-a \eta \sin \gamma
$$

instead of $\lambda_{1 / 2, x / y}$ [as defined in Eq. (C.27)]:

$$
\begin{aligned}
\left\langle i \mathcal{L}_{+}^{\mathrm{pp}} E_{\mathrm{rot}}\right\rangle_{t}=-\frac{3}{8 \sqrt{\pi}} a & n_{0} g(a)\left(\frac{m}{4 T_{\mathrm{x}}}\right)^{\frac{1}{2}}\left(\frac{m}{4 T_{\mathrm{y}}}\right)^{\frac{1}{2}}\left(\frac{I}{4 T_{\mathrm{rot}}}\right)^{\frac{1}{2}} \times \\
& \int_{\frac{\pi}{2}}^{\frac{3}{2} \pi} d \gamma \cos \gamma \int_{0}^{2 \pi} d \phi_{r} \frac{m}{2} \frac{\lambda_{1, r o t}+\mathcal{B} \lambda_{2, r o t}}{\sqrt{\mathcal{A C}^{5}}} .
\end{aligned}
$$

where $\mathcal{A}$ and $\mathcal{B}$ are defined in Eq. (C.25).

\section{Special Case 1: PP Contribution $\left\langle i \mathcal{L}_{+}^{\mathrm{pp}} E_{\mathrm{rot}}\right\rangle_{t}$ Assuming an Isotropic Translational Temperature $T_{\mathrm{tr}}$}

Assuming an isotropic temperature $T_{\mathrm{x}}=T_{\mathrm{y}}(t)=T_{\mathrm{tr}}(t)$ for all times simplifies Eq. (C.56) significantly, exactly as Eq. (C.26) is simplified to Eq. (C.29) in Sec. C.2.1. Since $\lambda_{1, \text { rot }}$ and $\lambda_{2}$ are already independent of $\phi_{r}$ integration over $\phi_{r}$ yields a factor $2 \pi$ and we get

$$
\left\langle i \mathcal{L}_{+}^{\mathrm{pp}} E_{\mathrm{rot}}\right\rangle_{t}=-\frac{3}{2} \sqrt{\frac{\pi}{m}} a n_{0} g(a) T_{\mathrm{tr}}^{\frac{3}{2}} x^{4} \int_{\frac{\pi}{2}}^{\frac{3}{2} \pi} d \gamma \frac{\sin ^{2} \gamma \cos \gamma}{\left(1-\left(x^{2}-1\right) \cos ^{2} \gamma\right)^{5 / 2}} 2 \eta\left(\frac{\eta}{q}-\frac{x^{2}-1}{x^{2}}\right) .
$$


Exactly like for $\left\langle i \mathcal{L}_{+}^{\mathrm{pp}} E_{\mathrm{x} / \mathrm{y}}\right\rangle_{t}$ up to this point we have not specified, whether we are going to use constant coefficients of restitution or Coulomb friction. All this is hidden in $\eta$ which is either a constant or a function of $\gamma$. For Coulomb friction we introduce the notation $\eta=$ $\frac{1+\alpha}{2} \mu \min \left\{\left|\cot \gamma_{0}\right|,|\cot \gamma|\right\} \equiv \min \left\{\eta_{0}, \frac{1+\alpha}{2} \mu|\cot \gamma|\right\}$ with $\eta_{0}:=\frac{q}{1+q} \frac{1+\beta_{0}}{2}$. Again, this leads to a splitting of the integral. Performing the integration over $\gamma$ yields

$$
\begin{aligned}
\frac{1}{2} \frac{d}{d t} T_{\text {rot }}=G T_{\mathrm{tr}}^{3 / 2} & \left\{\frac{1}{\left(1+x^{2} \cot ^{2} \gamma_{0}\right)^{3 / 2}}\left(\frac{\eta_{0}^{2} x^{2}}{2 q}-\left(x^{2}-1\right) \frac{\eta_{0}}{2}\left(1+x^{2} \cot ^{2} \gamma_{0}\right)\right)\right. \\
& \left.+\frac{\eta_{0}^{2} \tan ^{2} \gamma_{0}}{q}\left(1-\frac{1+\frac{3}{2} x^{2} \cot ^{2} \gamma_{0}}{\left(1+x^{2} \cot ^{2} \gamma_{0}\right)^{3 / 2}}\right)\right\}
\end{aligned}
$$

Finally, from Eqs. (C.31) and (C.58) the conversant reader may see the coefficients in Eqs. (10.23).

\section{Special Case 2: PP Contribution $\left\langle i \mathcal{L}_{+}^{\mathrm{pp}} E_{\mathrm{tot}}\right\rangle_{t}$ Assuming an Isotropic Total Temperature $T_{\text {tot }}$}

Adding Eqs. (C.31) and (C.58) and assuming an isotropic temperature $T_{\mathrm{x}}=T_{\mathrm{y}}=T_{\mathrm{tr}}=T_{\text {rot }}$ (which makes $x^{2}=1+T_{\text {rot }} /\left[q T_{\mathrm{tr}}\right]$ independent of the temperatures, $x^{2}=1+1 / q$ ) we find

$$
\begin{aligned}
\frac{3}{2} \frac{d}{d t} T_{\mathrm{tot}}= & H_{\mathrm{dr}}-G T_{\mathrm{tot}}^{3 / 2}\left\{A_{r}+\frac{\eta_{0} x^{2}}{2} \frac{1}{\left(1+x^{2} \cot ^{2} \gamma_{0}\right)^{1 / 2}}-\frac{\eta_{0}^{2}}{2} \frac{x^{4}}{\left(1+x^{2} \cot ^{2} \gamma_{0}\right)^{3 / 2}}\right. \\
& \left.-\eta_{0}^{2} x^{2} \tan ^{2} \gamma_{0}\left(1-\frac{1+\frac{3}{2} x^{2} \cot ^{2} \gamma_{0}}{\left(1+x^{2} \cot ^{2} \gamma_{0}\right)^{3 / 2}}\right)\right\}
\end{aligned}
$$

where, cf. Eq. (2.9), $x^{2} \cot ^{2} \gamma_{0}=\frac{q}{1+q} \frac{\left(1+\beta_{0}\right)^{2}}{(1+\alpha)^{2}} \frac{1}{\mu^{2}}$ because $T_{\text {tr }}=T_{\text {rot }}$. This can be easily solved for the stationary temperature in this approximation

$$
\begin{aligned}
T_{\text {tot }}^{\text {stat }}= & \left(\frac{H_{\mathrm{dr}}}{G}\right)^{\frac{2}{3}}\left\{A_{r}+\frac{\eta_{0} x^{2}}{2} \frac{1}{\left(1+x^{2} \cot ^{2} \gamma_{0}\right)^{1 / 2}}-\frac{\eta_{0}^{2}}{2} \frac{x^{4}}{\left(1+x^{2} \cot ^{2} \gamma_{0}\right)^{3 / 2}}\right. \\
& \left.-\eta_{0}^{2} x^{2} \tan ^{2} \gamma_{0}\left(1-\frac{1+\frac{3}{2} x^{2} \cot ^{2} \gamma_{0}}{\left(1+x^{2} \cot ^{2} \gamma_{0}\right)^{3 / 2}}\right)\right\}^{-\frac{2}{3}}
\end{aligned}
$$

Of course this is a very crude approximation since it is known [HZ97, LHMZ98] that energy equipartition is violated for inelastically colliding rough particles. 
How does the ratio of rotational and translational temperature behave reaching the steady state? Is there a constant ratio $T_{\text {rot }} / T_{\mathrm{tr}}$ ? To answer this question we divide Eq. (C.58) by Eq. (C.31) where $\frac{d T_{\text {rot }} / d t}{q T_{\text {tr }} / d t}=\frac{d T_{\text {rot }} / d t}{q T_{\text {tr }} / d t}$ to find

$$
\begin{aligned}
0= & \left(1+x^{2} \cot ^{2} \gamma_{0}\right)^{3 / 2} \frac{H_{\mathrm{dr}}}{G T_{\mathrm{tr}}^{3 / 2}}-A_{r}+\frac{\eta_{0}}{2} \frac{2-q}{q}\left(1+x^{2} \cot ^{2} \gamma_{0}\right) \\
& +\eta_{0}^{2}\left(1-\frac{2}{q^{2}\left(x^{2}-1\right)}\right)\left(\frac{x^{2}}{2}+\tan ^{2} \gamma_{0}\left(\left(1+x^{2} \cot ^{2} \gamma_{0}\right)^{3 / 2}-1-\frac{3}{2} x^{2} \cot ^{2} \gamma_{0}\right)\right)
\end{aligned}
$$

If $T_{\mathrm{rot}} / T_{\mathrm{tr}}$ approaches a constant value for late times close to the steady state then the corresponding $x$ solves this equation.

For a simple energy balance as in chapter 3 - but for rough particles - one has to add Eqs. (C.15) and (C.54) to find

$$
\frac{2}{m} \Delta E_{\mathrm{tot}}=-\frac{\left(1-\alpha^{2}\right)}{2}(\hat{\boldsymbol{r}} \cdot \boldsymbol{g})^{2}+2 \eta([1+1 / q] \eta-1)\left(|\boldsymbol{g}|^{2}-(\hat{\boldsymbol{r}} \cdot \boldsymbol{g})^{2}\right)
$$

This has to be averaged over all $\boldsymbol{v}$ and $\boldsymbol{\omega}$ and used instead of Eq. (3.4) to yield a corresponding simple energy argument. Therefore we calculate

$$
\left\langle\Delta E_{\mathrm{tot}}\right\rangle_{\{\boldsymbol{v}, \boldsymbol{\omega}\}}=\left(\frac{m}{4 \pi T_{\mathrm{tot}}}\right)^{\frac{3}{2}} \int d g_{x} d g_{y} d \omega_{\perp} \exp \left(-\frac{m\left|\boldsymbol{g}-\frac{a}{2} \boldsymbol{\omega}_{\perp}\right|^{2}+I\left|\boldsymbol{\omega}_{\perp}\right|^{2}}{4 T_{\mathrm{tot}}}\right) \Delta E_{\mathrm{tot}}
$$

Now we express $\boldsymbol{g}$ and $\boldsymbol{\omega}_{\perp}$ in polar coordinates, Eq. (C.21) and make the same substitution as after Eq. (C.25), $p_{1}:=\sqrt{\mathcal{C}} g$ for $g$ and $p_{2}:=\sqrt{\mathcal{A}}\left(\omega_{\perp}-\mathcal{B} g\right)$ for $\omega_{\perp}$ where the coefficients are defined in Eq. (C.25), $\widetilde{\mathcal{A}}^{*}=\frac{m a^{2}}{16 T_{\text {tot }}}(1+q), \widetilde{\mathcal{B}}^{*}=\frac{2}{a} \frac{\sin \gamma}{1+q}$, and $\widetilde{\mathcal{C}}^{*}=\frac{m}{4 T_{\text {tr }}} \frac{q}{1+q}\left(1-(1 / q) \cos ^{2} \gamma\right)$. We get

$$
\left\langle\Delta E_{\mathrm{tot}}\right\rangle_{\{\boldsymbol{v}, \boldsymbol{\omega}\}}=\left(\frac{m}{4 \pi T_{\mathrm{tot}}}\right)^{\frac{3}{2}} \int_{\frac{\pi}{2}}^{\frac{3}{2} \pi} d \gamma \int_{0}^{\infty} d p_{1} \int_{-\infty}^{\infty} d p_{2} p_{1} \frac{1}{\sqrt{\widetilde{\mathcal{A}}^{*} \widetilde{\mathcal{C}}^{*}}} \exp \left(-p_{1}^{2}-p_{2}^{2}\right) \frac{m}{2} \lambda_{\mathrm{tot}} \frac{p_{1}^{2}}{\widetilde{\mathcal{C}}^{*}}
$$

where $\lambda_{\text {tot }} g^{2}=\frac{2}{m} \Delta E_{\text {tot }}$, i.e. $\lambda_{\text {tot }}=-\frac{\left(1-\alpha^{2}\right)}{2} \cos ^{2} \gamma+2 \eta([1+1 / q] \eta-1) \sin ^{2} \gamma$. Thus we get

$$
\left\langle\Delta E_{\text {tot }}\right\rangle_{\{\boldsymbol{v}, \boldsymbol{\omega}\}}=\frac{m}{4 \pi}\left(\frac{m}{4 T_{\text {tot }}}\right)^{\frac{3}{2}} \int_{\frac{\pi}{2}}^{\frac{3}{2} \pi} d \gamma \frac{\lambda_{\text {tot }}}{\widetilde{\mathcal{C}}^{*} \sqrt{\widetilde{\mathcal{A}}^{*}}}
$$




\section{C.2.3. For Comparison: PP Contributions for 3D Systems}

For comparison, we quote the result for three dimensions [HHZ00, Her00]:

$$
\begin{aligned}
\frac{3}{2} \frac{d}{d t} T_{\mathrm{tr}}(t)= & H_{\mathrm{dr}}-G_{3 D} T_{\mathrm{tr}}^{3 / 2}\left\{A_{r}+\frac{\eta_{0}}{2} \frac{1-\eta_{0} x^{2}}{1+x^{2} \cot ^{2} \gamma_{0}}\right. \\
& \left.+\frac{\eta_{0}}{2}\left(\frac{\arctan \left(x \cot \gamma_{0}\right)}{x \cot \gamma_{0}}-\frac{1}{1+x^{2} \cot ^{2} \gamma_{0}}\right)\right\},
\end{aligned}
$$

and

$$
\begin{aligned}
\frac{3}{2} \frac{d}{d t} T_{\mathrm{rot}}(t) & =G_{3 D} T_{\mathrm{tr}}^{3 / 2}\left\{\eta_{0} \frac{1-\left(1-\frac{\eta_{0}}{q}\right) x^{2}}{1+x^{2} \cot ^{2} \gamma_{0}}\right. \\
- & \left.\frac{\eta_{0}}{2}\left(x^{2}-1\right)\left(\frac{\arctan \left(x \cot \gamma_{0}\right)}{x \cot \gamma_{0}}-\frac{1}{1+x^{2} \cot ^{2} \gamma_{0}}\right)\right\},
\end{aligned}
$$

where $G_{3 D}=8 \sqrt{\frac{\pi}{m}} a^{2} n_{0} g_{3 D}(a)$. The pair correlation at contact $g_{3 D}(a)$ can be estimated by the Carnahan-Starling approximation [CS69] for a 3D elastic hard sphere gas that depends on the volume fraction only: $g_{3 D}(a) \approx \frac{1-\phi / 2}{(1-\phi)^{3}}$.

\section{C.3. Particle-Wall Contribution $\left\langle i \mathcal{L}_{+}^{\mathrm{pw}} E_{\mathrm{x} / \mathrm{y} / \mathrm{tr} / \mathrm{rot}}\right\rangle_{t}$ to}

$$
\frac{d}{d t} T_{\mathrm{x} / \mathrm{y} / \operatorname{tr} / \operatorname{rot}}(t)
$$

Here we consider the change of $T_{\mathrm{x} / \mathrm{y}}$ due to collisions between a particle and wall at $x=0$ or $x=L_{x}$. The calculation is very similar to the one for the particle-particle contributions shown in Sec. C.2. Because we consider the system to be symmetric and homogeneous it is sufficient to consider $2 N$ collisions of particle 1 with the left wall. These considerations stand behind the equalities marked by $(*)$ and $(* *)$ in the following equation

$$
\begin{aligned}
\left\langle i \mathcal{L}_{+}^{\mathrm{pw}} E_{\mathrm{x} / \mathrm{y}}\right\rangle_{t} & =\left\langle i \mathcal{L}_{U+} E_{\mathrm{x} / \mathrm{y}}\right\rangle_{t}=\left\langle\sum_{l=1}^{2} \sum_{k=1}^{N} i T_{+}^{W_{l} k} E_{\mathrm{x} / \mathrm{y}}\right\rangle_{t} \stackrel{(*)}{=} 2\left\langle\sum_{k=1}^{N} i T_{+}^{W_{1} k} E_{\mathrm{x} / \mathrm{y}}\right\rangle_{t} \\
& =2\left\langle\sum_{k=1}^{N} i T_{+}^{W_{1} k} \frac{1}{N} \sum_{n=1}^{N} \frac{m}{2}\left|v_{n, x / y}\right|^{2}\right\rangle_{t}=\frac{2}{N}\left\langle\sum_{k=1}^{N} \frac{m}{2} i T_{+}^{W_{1} k}\left|v_{k, x / y}\right|^{2}\right\rangle_{t} \\
& =\frac{2}{N} \sum_{k=1}^{N} \frac{m}{2} \int \prod_{n=1}^{N} d r_{n}^{x} d r_{n}^{y} d v_{n}^{x} d v_{n}^{y} d \omega_{n}^{z} \rho(\boldsymbol{r}, \boldsymbol{v}, \boldsymbol{\omega}) i T_{+}^{W_{1} k}\left|v_{k, x / y}\right|^{2} \\
& \stackrel{(* *)}{=} \frac{2}{N} N \int \prod_{n=1}^{N} d^{2} r_{n} d^{2} v_{n} d \omega_{n}^{z} \rho(\boldsymbol{r}, \boldsymbol{v}, \boldsymbol{\omega}) i T_{+}^{W_{1} 1} \frac{m}{2}\left|v_{1, x / y}\right|^{2} .
\end{aligned}
$$


Using the definition of $d \Gamma$, Eq. (C.11), and the definition of $i T_{+}^{W_{1} 1}$, Eq. (C.4), we can write

$$
\left\langle i \mathcal{L}_{+}^{\mathrm{pw}} E_{\mathrm{x} / \mathrm{y}}\right\rangle_{t}=-\frac{2}{\int d \Gamma} \int d \Gamma\left(\hat{\boldsymbol{n}}_{1} \cdot \boldsymbol{v}_{1}\right) \Theta\left(-\hat{\boldsymbol{n}}_{1} \cdot \boldsymbol{v}_{1}\right) \delta\left(\left|\hat{\boldsymbol{n}}_{1} \cdot\left(\boldsymbol{r}_{1}-\boldsymbol{w}_{1}\right)\right|-\frac{a}{2}\right) \Delta E_{\mathrm{x} / \mathrm{y}}
$$

where $\hat{\boldsymbol{n}}_{1}$ is the normal vector of wall number 1 and $\boldsymbol{w}_{1}$ is a point on the surface of wall number 1 . For example, for the left wall being at $x=-L_{x} / 2, \hat{\boldsymbol{n}}_{1}=\hat{\boldsymbol{e}}_{x}$. For $\boldsymbol{w}_{1}$ one could use $\left(-L_{x} / 2,0,0\right)$. The change of partial energy $\Delta E_{\mathrm{x} / \mathrm{y}}:=\frac{m}{2}\left(b_{+}^{W_{1} 1}-1\right)\left|v_{1, x / y}\right|^{2}$ that results from a collision of particle 1 with wall number 1 depends only on the phase space variables of particle 1. Now we can perform the integrations over $d^{2} r_{2} \ldots d^{2} r_{N}, d^{2} v_{2} \ldots d^{2} v_{N}$ and $d \omega_{2} \ldots d \omega_{N}$. The integration over $d^{2} v_{2} \ldots d^{2} v_{N}$ and $d^{2} \omega_{2} \ldots d^{2} \omega_{N}$ are simple Gaussian integrals. To integrate over $d^{2} r_{2} \ldots d^{2} r_{N}$ we introduce two more two-dimensional integrals over two-dimensional $\delta$ functions, $\delta^{2}(\boldsymbol{r}):=\delta\left(r_{x}\right) \delta\left(r_{y}\right), \int d^{2} R_{1} d^{2} R_{2} \delta^{2}\left(\boldsymbol{R}_{1}-\boldsymbol{r}_{1}\right) \delta^{2}\left(\boldsymbol{R}_{2}-\boldsymbol{r}_{2}\right)$. Using $\boldsymbol{R}_{12}:=\boldsymbol{R}_{1}-\boldsymbol{R}_{2}$ and the definition of the pair correlation function, Eq. (C.12), we obtain

$$
\begin{aligned}
\left\langle i \mathcal{L}_{+}^{\mathrm{pw}} E_{\mathrm{x} / \mathrm{y}}\right\rangle_{t}=-\frac{2}{A^{2}} & \left(\frac{m}{2 \pi T_{\mathrm{x}}}\right)^{\frac{1}{2}}\left(\frac{m}{2 \pi T_{\mathrm{y}}}\right)^{\frac{1}{2}}\left(\frac{I}{2 \pi T_{\mathrm{rot}}}\right)^{\frac{1}{2}} \int d^{2} R_{1} d^{2} R_{2} d^{2} v_{1} d \omega_{1} \times \\
& g\left(\left|\boldsymbol{R}_{12}\right|\right) \exp \left(-\frac{m}{2}\left[\frac{v_{1, x}^{2}}{T_{\mathrm{x}}}+\frac{v_{1, y}^{2}}{T_{\mathrm{y}}}\right]-\frac{I}{2} \frac{\omega_{1}^{2}}{T_{\mathrm{rot}}}\right) \times \\
& \left(\hat{\boldsymbol{n}}_{1} \cdot \boldsymbol{v}_{1}\right) \delta\left(\left|\hat{\boldsymbol{n}}_{1} \cdot\left(\boldsymbol{r}_{1}-\boldsymbol{w}_{1}\right)\right|-\frac{a}{2}\right) \Theta\left(-\hat{\boldsymbol{n}}_{1} \cdot \hat{\boldsymbol{v}}_{1}\right) \Delta E_{\mathrm{x} / \mathrm{y}} .
\end{aligned}
$$

where

$$
\begin{aligned}
\frac{2}{m} \Delta E_{\mathrm{x} / \mathrm{y}}:= & \left(b_{+}^{W 1}-1\right)\left|v_{x / y}\right|^{2}=\left|v_{x / y}^{\prime}\right|^{2}-\left|v_{x / y}\right|^{2} \stackrel{(2.14)}{=}\left|v_{x / y}+2 \Delta v_{x / y}\right|^{2}-\left|v_{x / y}\right|^{2} \\
= & 4\left|\Delta v_{x / y}\right|^{2}+4 v_{x / y} \Delta v_{x / y} \\
& \stackrel{(2.15)}{=}\left(1+\alpha_{\mathrm{W}}\right)^{2}\left(\hat{\boldsymbol{n}}_{1} \cdot \boldsymbol{v}\right)^{2}\left(\hat{\boldsymbol{n}}_{1} \cdot \hat{\boldsymbol{e}}_{x / y}\right)^{2}+4 \eta^{2}\left[\left(\boldsymbol{v}-\hat{\boldsymbol{n}}_{1}\left[\hat{\boldsymbol{n}}_{1} \cdot \boldsymbol{v}\right]+\left(\frac{a}{2}\right)\left[\hat{\boldsymbol{n}}_{1} \times \boldsymbol{\omega}\right]\right) \cdot \hat{\boldsymbol{e}}_{x / y}\right]^{2} \\
& +4 \eta\left(1+\alpha_{\mathrm{w}}\right)\left(\hat{\boldsymbol{n}}_{1} \cdot \boldsymbol{v}\right)\left(\hat{\boldsymbol{n}}_{1} \cdot \hat{\boldsymbol{e}}_{x / y}\right)\left[\left(\boldsymbol{v}-\hat{\boldsymbol{n}}_{1}\left[\hat{\boldsymbol{n}}_{1} \cdot \boldsymbol{v}\right]+\left(\frac{a}{2}\right)\left[\hat{\boldsymbol{n}}_{1} \times \boldsymbol{\omega}\right]\right) \cdot \hat{\boldsymbol{e}}_{x / y}\right] \\
& -2\left(1+\alpha_{\mathrm{w}}\right)\left(\hat{\boldsymbol{n}}_{1} \cdot \boldsymbol{v}\right)\left(\hat{\boldsymbol{n}}_{1} \cdot \hat{\boldsymbol{e}}_{x / y}\right)\left(\hat{\boldsymbol{v}} \cdot \hat{\boldsymbol{e}}_{x / y}\right) \\
& -4 \eta\left[\left(\boldsymbol{v}-\hat{\boldsymbol{n}}_{1}\left[\hat{\boldsymbol{n}}_{1} \cdot \boldsymbol{v}\right]+\left(\frac{a}{2}\right)\left[\hat{\boldsymbol{n}}_{1} \times \boldsymbol{\omega}\right]\right) \cdot \hat{\boldsymbol{e}}_{x / y}\right]\left(\hat{\boldsymbol{v}} \cdot \hat{\boldsymbol{e}}_{x / y}\right) .
\end{aligned}
$$

For walls in the $x$-direction we get $\hat{\boldsymbol{n}}_{1}=\hat{\boldsymbol{e}}_{\boldsymbol{x}}$ and thus $\Delta E_{\mathrm{tr}}$ simplifies to

$$
\begin{aligned}
\frac{2}{m} \Delta E_{\mathrm{tr}}= & -\left(1-\alpha_{\mathrm{w}}^{2}\right)\left(\hat{\boldsymbol{n}}_{1} \cdot \boldsymbol{v}\right)^{2}+4 \eta_{\mathrm{w}}\left(\eta_{\mathrm{w}}-1\right)\left(|\boldsymbol{v}|^{2}-\left(\hat{\boldsymbol{n}}_{1} \cdot \boldsymbol{v}\right)^{2}\right) \\
& +\eta_{\mathrm{w}}^{2} a^{2}\left|\hat{\boldsymbol{n}}_{1} \times \boldsymbol{\omega}\right|^{2}+2 \eta_{\mathrm{w}}\left(2 \eta_{\mathrm{w}}-1\right) a\left(\hat{\boldsymbol{n}}_{1} \times \boldsymbol{\omega}\right) \cdot \boldsymbol{v} .
\end{aligned}
$$

In general, $\eta_{\mathrm{w}}=\frac{q}{1+q} \frac{1+\beta}{2}$, where $\beta=\beta(|\cot \gamma|) \stackrel{(2.8)}{=}$ const $\min \left(|\cot \gamma|,\left|\cot \gamma_{0}\right|\right)$ is a function of $|\cot \gamma|:=\frac{\left|\boldsymbol{g} \cdot \hat{\boldsymbol{n}}_{1}\right|}{\left|\boldsymbol{g} \times \hat{\boldsymbol{n}}_{1}\right|}$ and $\boldsymbol{g} \stackrel{(2.1)}{=} \boldsymbol{v}+\frac{a}{2} \hat{\boldsymbol{n}}_{1} \times \boldsymbol{\omega}$ is a function of $\hat{\boldsymbol{n}}_{1}, \boldsymbol{v}$ and $\boldsymbol{\omega}$.

Now we could continue similarly as in Sec. C.2.1 by performing substitution (C.17). 


\section{C.3.1. PW Contribution $\left\langle i \mathcal{L}_{+}^{\mathrm{pw}} E_{\mathrm{x} / \mathrm{y}}\right\rangle_{t}$ for Smooth Particle-Wall Interactions}

For smooth particle-wall interactions the rotational energy remains unchanged when a particle collides with a wall and we have to consider the change of translational energy only. In addition $\eta_{\mathrm{w}}=0$. We want to be more specific about the walls in order to improve the readability. We choose our coordinate system such that the wall is at $x=0, \hat{\boldsymbol{n}}_{1}=\hat{\boldsymbol{e}}_{x}, \boldsymbol{w}=(0,0,0)$. We note that $\int d^{2} R_{1} d^{2} R_{2} g\left(\left|\boldsymbol{R}_{12}\right|\right) \delta\left(R_{1}^{x}-\frac{a}{2}\right)=L_{y} V$, as can be easily seen when writing down and inserting $g\left(\boldsymbol{R}_{12} \mid\right) / V^{2}$ : The integrals over $d^{2} R_{1}$ and $d^{2} R_{2}$ can be solved trivially (in the numerator and in the denominator). Then, we substitute $\widetilde{\boldsymbol{R}}_{i}:=\boldsymbol{r}_{i}-\boldsymbol{r}_{1}$ for $i=2$..n and $\widetilde{\boldsymbol{R}}_{1}:=\boldsymbol{r}_{1}$. That way, all the integrals over Heaviside's step functions cancel and we get $\int d^{2} \widetilde{R}_{1} \delta\left(\widetilde{R}_{1}^{x}-\frac{a}{2}\right) / \int d^{2} \widetilde{R}_{1}=L_{y} / V=1 / L_{x}$.

We ommit the index 1 of $\boldsymbol{v}_{1}$ and $\omega_{1}$ and get

$$
\begin{aligned}
\left\langle i \mathcal{L}_{+}^{\mathrm{pw}} E_{\mathrm{x} / \mathrm{y}}\right\rangle_{t}=- & \frac{2}{L_{x}}\left(\frac{m}{2 \pi T_{\mathrm{x}}}\right)^{\frac{1}{2}}\left(\frac{m}{2 \pi T_{\mathrm{y}}}\right)^{\frac{1}{2}}\left(\frac{I}{2 \pi T_{\mathrm{rot}}}\right)^{\frac{1}{2}} \int d^{2} v d \omega \times \\
& \quad \exp \left(-\frac{m}{2}\left[\frac{v_{1, x}^{2}}{T_{\mathrm{x}}}+\frac{v_{1, y}^{2}}{T_{\mathrm{y}}}\right]-\frac{I}{2} \frac{\omega_{1}^{2}}{T_{\mathrm{rot}}}\right) v_{x} \Theta\left(-v_{x}\right) \Delta E_{\mathrm{x} / \mathrm{y}}(\boldsymbol{v}, \boldsymbol{\omega}) .
\end{aligned}
$$

Here, the integration over $d \omega$ can be performed trivially, and (since $\eta_{\mathrm{w}}=0$ ) the change of energy simplifies to

$$
\Delta E_{\mathrm{x}}(\boldsymbol{v})=\frac{m}{2}\left[-\left(1-\alpha_{\mathrm{w}}^{2}\right) v_{x}^{2}-2 \alpha_{\mathrm{w}} v_{\mathrm{dr}} v_{x}+v_{\mathrm{dr}}^{2}\right]=\Delta E_{\mathrm{x}}\left(v_{x}, v_{\mathrm{dr}}\right), \quad \Delta E_{\mathrm{y}}(\boldsymbol{v})=0 .
$$

Therefore we get

$$
\begin{aligned}
\left\langle i \mathcal{L}_{+}^{\mathrm{pw}} E_{\mathrm{y}}\right\rangle_{t}= & 0 \quad \text { and } \\
\left\langle i \mathcal{L}_{+}^{\mathrm{pw}} E_{\mathrm{x}}\right\rangle_{t}= & -\frac{2}{L_{x}}\left(\frac{m}{2 \pi T_{\mathrm{x}}(t)}\right)^{\frac{1}{2}}\left(\frac{m}{2 \pi T_{\mathrm{y}}(t)}\right)^{\frac{1}{2}}\left(\frac{I}{2 \pi T_{\mathrm{rot}}(t)}\right)^{\frac{1}{2}} \int d^{2} v d \omega \times \\
& \quad \exp \left(\frac{-\frac{m}{2}\left(\frac{\left|v^{x}\right|^{2}}{T_{x}(t)}+\frac{\left|v^{y}\right|^{2}}{T_{y}(t)}\right)}{1}+\frac{\left.-\frac{I}{2}|\boldsymbol{\omega}|^{2}\right)}{T_{\mathrm{rot}}(t)}\right) v_{x} \Theta\left(-v_{x}\right) \Delta E_{\mathrm{x}} \\
= & -\frac{2}{L_{x}}\left(\frac{m}{2 \pi T_{\mathrm{x}}(t)}\right)^{\frac{1}{2}} \int_{-\infty}^{0} d v_{x} \exp \left(-\frac{m}{2} \frac{v_{x}^{2}}{T_{x}(t)}\right) v_{x} \Delta E_{\mathrm{x}}\left(v_{x}, v_{\mathrm{dr}}\right) \\
= & \frac{m}{L_{x}}\left(\frac{m}{2 \pi T_{\mathrm{x}}(t)}\right)^{\frac{1}{2}} \int_{0}^{\infty} d v_{x} \exp \left(-\frac{m}{2} \frac{v_{x}^{2}}{T_{x}(t)}\right)\left[-\left(1-\alpha_{\mathrm{w}}^{2}\right) v_{x}^{3}+2 \alpha_{\mathrm{w}} v_{\mathrm{dr}} v_{x}^{2}+v_{\mathrm{dr}}^{2} v_{x}\right] \\
= & \frac{m v_{\mathrm{dr}}^{2}}{\sqrt{2 \pi} L_{x}} \sqrt{\frac{T_{\mathrm{x}}}{m}}\left[1+\sqrt{2 \pi} \alpha_{\mathrm{w}} \sqrt{\frac{T_{x}(t)}{m v_{\mathrm{dr}}^{2}}}-2\left(1-\alpha_{\mathrm{w}}^{2}\right)\left(\frac{T_{x}(t)}{m v_{\mathrm{dr}}^{2}}\right)\right] .
\end{aligned}
$$


Written in a form that is well-defined also for $v_{\mathrm{dr}}=0$ the final result is

$$
\left\langle i \mathcal{L}_{+}^{\mathrm{pw}} E_{\mathrm{x}}\right\rangle_{t}=\frac{T_{\mathrm{x}}^{\frac{1}{2}}}{L_{x}} \frac{1}{\sqrt{2 \pi m}}\left[m v_{\mathrm{dr}}^{2}+\sqrt{2 \pi} \alpha_{\mathrm{w}} m \sqrt{\frac{T_{x}(t)}{m}} v_{\mathrm{dr}}-2\left(1-\alpha_{\mathrm{w}}^{2}\right) T_{x}(t)\right] .
$$

\section{C.4. Total Time Derivative of Partial Temperatures $\frac{d}{d t} T_{\mathrm{x} / \mathrm{y}}(t)$ for Smooth Particles}

For a system with 2 identical walls in the $x$-direction and periodic boundary conditions in the $y$-direction we insert Eqs. (C.49) and (C.73) into Eq. (C.9) to obtain:

$$
\begin{aligned}
\frac{1}{2} \frac{d}{d t} T_{\mathrm{x}}(t)= & \frac{d}{d t}\left\langle E_{\mathrm{x}}\right\rangle_{t}=\left\langle i \mathcal{L}_{+}^{\mathrm{pp}} E_{\mathrm{x}}+\left\langle i \mathcal{L}_{+}^{\mathrm{pw}} E_{\mathrm{x}}\right\rangle_{t}\right. \\
= & -\frac{a n_{0} g(a)}{2 \pi} \sqrt{\frac{\pi}{m}} T_{\mathrm{tr}}^{\frac{3}{2}} \times \\
& \left\{\Theta\left(T_{\mathrm{x}}-T_{\mathrm{y}}\right)\left(\frac{T_{\mathrm{y}}}{T_{\mathrm{tr}}}\right)^{\frac{3}{2}} \sqrt{\frac{T_{\mathrm{y}}}{T_{\mathrm{x}}}}\left(\mathcal{F}_{y / x} f_{1}\left(k_{x y}\right) \mp \mathcal{F}_{0} f_{c}\left(k_{x y}\right)\right)\right. \\
& \left.+\Theta\left(T_{\mathrm{y}}-T_{\mathrm{x}}\right)\left(\frac{T_{\mathrm{x}}}{T_{\mathrm{tr}}}\right)^{\frac{3}{2}} \sqrt{\frac{T_{\mathrm{x}}}{T_{\mathrm{y}}}}\left(\mathcal{F}_{x / y} f_{1}\left(k_{y x}\right) \pm \mathcal{F}_{0} f_{c}\left(k_{y x}\right)\right)\right\} \\
& +\frac{T_{\mathrm{x}}^{\frac{1}{2}}}{L_{x}} \frac{1}{\sqrt{2 \pi m}}\left[m v_{\mathrm{dr}}^{2}+\sqrt{2 \pi} \alpha_{\mathrm{w}} m \sqrt{\frac{T_{x}}{m}} v_{\mathrm{dr}}-2\left(1-\alpha_{\mathrm{w}}^{2}\right) T_{x}\right] \\
= & m\left(\frac{T_{\mathrm{tr}}}{m}\right)^{\frac{3}{2}}\left(\frac { 1 } { \sqrt { 2 \pi } } \frac { 1 } { L _ { x } } \sqrt { \frac { T _ { \mathrm { x } } } { T _ { \mathrm { tr } } } } \left[\frac{m v_{\mathrm{dr}}^{2}}{T_{\mathrm{tr}}}+\sqrt{2 \pi} \alpha_{\mathrm{w}} \sqrt{\frac{T_{x}}{T_{\mathrm{tr}}}} \sqrt{\left.\frac{m v_{\mathrm{dr}}^{2}}{T_{\mathrm{tr}}}-2\left(1-\alpha_{\mathrm{w}}^{2}\right) \frac{T_{x}}{T_{\mathrm{tr}}}\right]}\right.\right. \\
& -\frac{a n_{0} g(a)}{2 \sqrt{\pi}}\left\{\Theta\left(T_{\mathrm{x}}-T_{\mathrm{y}}\right)\left(\frac{T_{\mathrm{y}}}{T_{\mathrm{tr}}}\right)^{\frac{3}{2}} \sqrt{\frac{T_{\mathrm{y}}}{T_{\mathrm{x}}}}\left(\mathcal{F}_{y / x} f_{1}\left(k_{x y}\right) \mp \mathcal{F}_{0} f_{c}\left(k_{x y}\right)\right)\right. \\
& \left.\left.+\Theta\left(T_{\mathrm{y}}-T_{\mathrm{x}}\right)\left(\frac{T_{\mathrm{x}}}{T_{\mathrm{tr}}}\right)^{\frac{3}{2}} \sqrt{\frac{T_{\mathrm{x}}}{T_{\mathrm{y}}}}\left(\mathcal{F}_{x / y} f_{1}\left(k_{y x}\right) \pm \mathcal{F}_{0} f_{c}\left(k_{y x}\right)\right)\right\}\right)
\end{aligned}
$$

$$
\begin{aligned}
\frac{1}{2} \frac{d}{d t} T_{\mathrm{y}}(t)= & \frac{d}{d t}\left\langle E_{\mathrm{y}}\right\rangle_{t}=\left\langle i \mathcal{L}_{+}^{\mathrm{pp}} E_{\mathrm{y}}\right\rangle_{t}+\left\langle i \mathcal{L}_{+}^{\mathrm{pw}} E_{\mathrm{y}}\right\rangle_{t} \\
& =-\frac{a n_{0} g(a)}{2 \pi} \sqrt{\frac{\pi}{m}} T_{\mathrm{tr}}^{\frac{3}{2}} \times \\
& \left\{\Theta\left(T_{\mathrm{x}}-T_{\mathrm{y}}\right)\left(\frac{T_{\mathrm{y}}}{T_{\mathrm{tr}}}\right)^{\frac{3}{2}} \sqrt{\frac{T_{\mathrm{y}}}{T_{\mathrm{x}}}}\left(\mathcal{F}_{y / x} f_{1}\left(k_{x y}\right) \mp \mathcal{F}_{0} f_{c}\left(k_{x y}\right)\right)\right. \\
+ & \left.\Theta\left(T_{\mathrm{y}}-T_{\mathrm{x}}\right)\left(\frac{T_{\mathrm{x}}}{T_{\mathrm{tr}}}\right)^{\frac{3}{2}} \sqrt{\frac{T_{\mathrm{x}}}{T_{\mathrm{y}}}}\left(\mathcal{F}_{x / y} f_{1}\left(k_{y x}\right) \pm \mathcal{F}_{0} f_{c}\left(k_{y x}\right)\right)\right\}+0
\end{aligned}
$$




\section{C.5. Energy Balance for Smooth Particles in the Stationary State}

Assuming an isotropic temperature $T_{\mathrm{tr}}=T_{\mathrm{x}}=T_{\mathrm{y}}$ we find by adding Eqs. (C.74) and (C.75)

$$
\begin{aligned}
\frac{1}{2} \frac{d}{d t} T_{\mathrm{tr}}(t)= & \frac{d}{d t}\left\langle E_{\mathrm{tr}}\right\rangle_{t}=\frac{d}{d t}\left\langle E_{\mathrm{x}}\right\rangle_{t}+\frac{d}{d t}\left\langle E_{\mathrm{y}}\right\rangle_{t}=\left\langle i \mathcal{L}_{+}^{\mathrm{pp}} E_{\mathrm{x}}+\left\langle i \mathcal{L}_{+}^{\mathrm{pw}} E_{\mathrm{x}}\right\rangle_{t}+\left\langle i \mathcal{L}_{+}^{\mathrm{pp}} E_{\mathrm{y}}\right\rangle_{t}\right. \\
= & m\left(\frac{T_{\mathrm{tr}}}{m}\right)^{\frac{3}{2}}\left(\frac{1}{\sqrt{2 \pi}} \frac{1}{L_{x}}\left[\frac{m v_{\mathrm{dr}}^{2}}{T_{\mathrm{tr}}}+\sqrt{2 \pi} \alpha_{\mathrm{w}} \sqrt{\frac{m v_{\mathrm{dr}}^{2}}{T_{\mathrm{tr}}}}-2\left(1-\alpha_{\mathrm{w}}^{2}\right)\right]\right. \\
& \left.-\frac{a n_{0} g(a)}{2 \sqrt{\pi}}\left(\mathcal{F}_{x}+\mathcal{F}_{y}\right) f_{1}(0)\right) \\
= & m\left(\frac{T_{\mathrm{tr}}}{m}\right)^{\frac{3}{2}}\left(\frac{1}{\sqrt{2 \pi}} \frac{1}{L_{x}}\left[\frac{m v_{\mathrm{dr}}^{2}}{T_{\mathrm{tr}}}+\sqrt{2 \pi} \alpha_{\mathrm{w}} \sqrt{\frac{m v_{\mathrm{dr}}^{2}}{T_{\mathrm{tr}}}}-2\left(1-\alpha_{\mathrm{w}}^{2}\right)\right]\right. \\
& \left.-a n_{0} g(a) \sqrt{\pi}\left(1-\alpha^{2}\right)\right)
\end{aligned}
$$

For the stationary state the time derivative must vanish which leads to the following quadratic equation in $\sqrt{T_{\mathrm{tr}}}$ (assuming $T_{\mathrm{tr}} \neq 0$ )

$$
0=\frac{1}{\sqrt{2 \pi}} \frac{1}{L_{x}}\left[\frac{m v_{\mathrm{dr}}^{2}}{T_{\mathrm{tr}}}+\sqrt{2 \pi} \alpha_{\mathrm{w}} \sqrt{\frac{m v_{\mathrm{dr}}^{2}}{T_{\mathrm{tr}}}}-2\left(1-\alpha_{\mathrm{w}}^{2}\right)\right]-a n_{0} g(a) \sqrt{\pi}\left(1-\alpha^{2}\right) .
$$

This can be simplified and multiplied by $T_{\mathrm{tr}}$ to yield

$$
0=-\left[2\left(1-\alpha_{\mathrm{w}}^{2}\right)+\sqrt{2} \pi a n_{0} L_{x} g(a)\left(1-\alpha^{2}\right)\right]{\sqrt{T_{\mathrm{tr}}}}^{2}+\alpha_{\mathrm{w}} \sqrt{2 \pi m v_{\mathrm{dr}}^{2}} \sqrt{T_{\mathrm{tr}}}+m v_{\mathrm{dr}}^{2} .
$$

For $v_{\mathrm{dr}}=0$ this implies $T_{\mathrm{tr}}=0$ or $\varepsilon_{\mathrm{eff}}=0$ where $\varepsilon_{\mathrm{eff}}$ has been defined in Eq. (3.9). For a discussion cf. chapter 3 .

For $\varepsilon_{\text {eff }} \neq 0$ Eq. (C.78) can be re-written as

$$
0={\sqrt{T_{\mathrm{tr}}}}^{2}-2 \alpha_{\mathrm{w}} \sqrt{m v_{\mathrm{dr}}^{2}} \sqrt{\frac{\pi}{2}} \frac{\left(\varepsilon_{\mathrm{eff}} \psi\right)^{-1}}{\pi} \sqrt{T_{\mathrm{tr}}}-m v_{\mathrm{dr}}^{2} \frac{\left(\varepsilon_{\mathrm{eff}} \psi\right)^{-1}}{\pi}
$$

with $\psi$ and $\varepsilon_{\text {eff }}$ as defined in Eq. (3.9).

For $\varepsilon_{\text {eff }}>0$ we find

$$
\sqrt{T_{\mathrm{tr}}}=\sqrt{m v_{\mathrm{dr}}^{2}} \sqrt{\frac{\pi}{2}} \frac{\sqrt{\left(\varepsilon_{\mathrm{eff}} \psi\right)^{-1}}}{\pi}\left[\sqrt{\left(\varepsilon_{\mathrm{eff}} \psi\right)^{-1} \alpha_{\mathrm{w}}^{2}}+\sqrt{2+\left(\varepsilon_{\mathrm{eff}} \psi\right)^{-1} \alpha_{\mathrm{w}}^{2}}\right]
$$


or

$$
T_{\mathrm{tr}}=\frac{m v_{\mathrm{dr}}^{2}}{2 \pi} \psi_{\mathrm{eff}}^{-2}\left(1+\sqrt{1+2 \psi_{\mathrm{eff}} / \alpha_{\mathrm{w}}}\right)^{2}
$$

with $\psi_{\text {eff }}$ as defined in Eq. (B.3) - and using $\widetilde{\alpha}_{\mathrm{w}}=\alpha_{\mathrm{w}}$ since we have calculated the case of identical walls here.

\section{C.6. Why and How Do the Simple and the Refined Energy Balance Differ?}

The result for the stationary temperature using the Liouville-Operator approach, Eq. (C.81), differs from the one obtained by the simple energy balance argument in chapter 3, Eq. (3.12). This is due to the fact that in chapter 3 we averaged the collision frequency independently of the change of energy instead of calculating the average of the fluctuating collision frequency times the change of energy, i.e we calculated

$$
\begin{gathered}
\left\langle v_{x} \cdot \Theta\left(v_{x}\right)\right\rangle \cdot\langle 1\rangle=\sqrt{\frac{T_{\mathrm{tr}}}{2 \pi}} \quad \text { instead of } \quad\left\langle v_{x} \cdot 1 \cdot \Theta\left(v_{x}\right)\right\rangle=\sqrt{\frac{T_{\mathrm{tr}}}{2 \pi}} \quad \text { (ok!), } \\
\left\langle v_{x} \cdot \Theta\left(v_{x}\right)\right\rangle \cdot\left\langle v_{x}\right\rangle=\frac{T_{\mathrm{tr}}}{\frac{2}{\pi}} \quad \text { instead of } \quad\left\langle v_{x} \cdot v_{x} \cdot \Theta\left(v_{x}\right)\right\rangle=\frac{T_{\mathrm{tr}}}{\pi} \quad \text { (not ok), and } \\
\left\langle v_{x} \cdot \Theta\left(v_{x}\right)\right\rangle \cdot\left\langle v_{x}^{2}\right\rangle=\sqrt{\frac{T_{\mathrm{tr}}^{3}}{2 \pi}} \quad \text { instead of } \quad\left\langle v_{x} \cdot v_{x}^{2} \cdot \Theta\left(v_{x}\right)\right\rangle=2 \sqrt{\frac{T_{\mathrm{tr}}^{3}}{2 \pi}} \quad \text { (not ok). }
\end{gathered}
$$

Indeed: We can obtain exactly the same result as calculated using the Pseudo-LiouvilleOperator approach, Eq. (C.81), from the simple calculation leading to Eq. (3.12) by replacing

$$
{\sqrt{T_{\mathrm{tr}}}}^{2} \rightarrow \frac{\pi}{2}{\sqrt{T_{\mathrm{tr}}}}^{2} \text { and }{\sqrt{T_{\mathrm{tr}}}}^{3} \rightarrow 2{\sqrt{T_{\mathrm{tr}}}}^{3}
$$

in Eq. (3.7). Doing so for two distinct walls, from appendix B we see that instead of Eq. (B.4) we obtain the final result of the refined theory for two distinct walls and perfectly smooth particles and walls

$$
T_{\mathrm{tr}}=\frac{m v_{\mathrm{dr}}^{2}}{2 \pi} \psi_{\mathrm{eff}}^{-2}\left(1+\sqrt{1+2 \psi_{\mathrm{eff}} / \widetilde{\alpha}_{\mathrm{w}}}\right)^{2},
$$

with the definitions from Eqs. (3.9), (B.2), and (B.3). 


\section{C.7. Artifact of the Walton Model: Surviving Rotational Energy without Driving}

For freely cooling 3D systems it has been found [HHZ00] that for sufficiently small $\mu$ the Walton model predicts the rotational energy to survive while the translational energy keeps decreasing according to Haff's law [Haf83] for all times. While it has been argued [HHZ00] that probably this behavior is an artifact due to the assumption of zero cohesion forces between the particles it is not completely impossible that a surviving rotational energy might be observable for certain materials. For 2D systems we find the same behavior in simulations as well as in the MF theory of the full Walton model, model E. Model A, B or D do not predict a surviving rotational energy - in contradiction to simulations: In models A, B, and D the coefficients in Eq. (10.4) are constants and thus they all predict qualitatively similar behavior.

As can be seen from Eq. (10.26) for $H_{\mathrm{dr}}=0$ the asymptotic behavior of the translational temperature will follow Haff's law [Haf83], namely $T_{\mathrm{tr}}=b t^{-2}$ with some constant $b>0$. If we insert this solution for $T_{\mathrm{tr}}$, Eq. (10.26), with $H_{\mathrm{dr}}=0$ into the differential equation for $T_{\mathrm{rot}}$, Eq. (C.58), we see that $R$ must increase for all times for sufficiently small $\mu$.

Proof: First we show that $R$ cannot approach zero by contradiction: If $R$ approached zero (that means $x \rightarrow 1$ ) then, for small enough $\mu$ (and small enough $R$ ), the combination $R \widetilde{C}_{\mu}(R)$, as it appears in Eq. (10.4), would become negligible in comparison with $\widetilde{B}_{\mu}^{\prime}(R)$, cf. to Eq. (10.25). Therefore, $T_{\text {rot }}$ would increase while $T_{\text {tr }}$ would continue to decrease. Thus $R$ would increase again which is contrary to the assumption that $R$ would approach zero.

Now we show that $R$ cannot reach an asymptotic value for sufficiently small $\mu$ (as it would happen for large enough $\mu$, especially for $\mu \rightarrow \infty$ ), again by contradiction: Let us assume $R$ reaches an asymptotic value (that means $R=$ const $<\infty$ ). Then we see from $\widetilde{B}_{\mu}^{\prime}(R)$ and $\widetilde{C}_{\mu}(R)$ in Eqs. (10.25) with $T_{\text {rot }}(t)=R T_{\text {tr }}(t)=b R t^{-2}$ that for sufficiently small $\mu \ll 1 \mathrm{Eq}$. (C.58) simplifies to

$$
\begin{aligned}
-2 \frac{b R}{t^{3}} & =\frac{d}{d t} \frac{b R}{t^{2}}=\frac{d}{d t} T_{\mathrm{rot}}(t) T_{\mathrm{tr}}^{3 / 2} \\
& =-2 G\left(\frac{b}{t^{2}}\right)^{\frac{3}{2}}\left(\frac{1+b}{4} \frac{\frac{R}{q}}{\sqrt{1+\frac{R}{q}}} \mu+\mathcal{O}\left(\mu^{2}\right)\right)
\end{aligned}
$$

which simplifies to $\sqrt{1+\frac{R}{q}}=G \sqrt{b} \frac{1+b}{4 q} \mu$. If $\mu<\frac{4 q}{1+b} \frac{1}{G \sqrt{b}}$ there is no positive $R$ that solves this equation. Therefore, the ansatz $T_{\text {rot }}(t)=R T_{\text {tr }}(t)$ with a constant $R$ for late times must be wrong.

Instead, for sufficiently small $\mu, R \rightarrow \infty$. 
To lowest order in $\mu$ for late times ( $R \gg 1$ ), Eq. (C.58) or, equivalently, Eqs. (10.4) with Eqs. (10.25) reads $\frac{d}{d t} T_{\text {rot }}(t)=-2 G \frac{1+b}{4 \sqrt{q}} \mu T_{\text {tr }}(t) T_{\text {rot }}^{\frac{1}{2}}(t)=-2 G b \frac{1+b}{4 \sqrt{q}} \mu T_{\text {rot }}^{\frac{1}{2}}(t) t^{-2}$. The solution for this differential equation is $\sqrt{T_{\text {rot }}(t)}=$ const $+G b \frac{1+b}{4 \sqrt{q}} \mu t^{-1}$. This prediction has been confirmed by simulations (not shown). The three-dimensional equivalent can be found in Ref. [HHZ00]. 


\section{Bibliography}

[AGZ98] T. Aspelmeier, G. Giese, and A. Zippelius. Cooling dynamics of a dilute gas of inelastic rods: A many particle simulation. Phys. Rev. E, 57:857, 1998. 2

$\left[\mathrm{BBR}^{+} 02\right]$ A. Barrat, T. Biben, Z. Racz, E. Trizac, and F. van Wijland. On the velocity distributions of the one-dimensional inelastic gas. J. Phys. A: Math. Gen., 66(35):463-480, 2002. 3

[BC98] J. J. Brey and D. Cubero. Steady state of a fluidized granular medium between two walls at the same temperature. Phys. Rev. E, 57(2):2019-2029, 1998. 2, 3

[BDKS98] J. J. Brey, J. W. Dufty, C. S. Kim, and A. Santos. Hydrodynamics for granular flow at low density. Phys. Rev. E, 58(4):4638, 1998. 1, 2

[BDS97] J. J. Brey, J. W. Dufty, and A. Santos. Dissipative dynamics for hard spheres. J. Stat. Phys., 87(5/6):1051-1067, 1997. 3, 28

[BDS99] J. J. Brey, J. W. Dufty, and A. Santos. Kinetic models for granular flow. J. Stat. Phys., 97:281-322, 1999. 2

[Bir94] G. A. Bird. Molecular Gas Dynamics and the Direct Simulation of Gas Flows. Clarendon, Oxford, 1994. 3

[BMD96] J. Javier Brey, F. Moreno, and James W. Dufty. Model kinetic equation for lowdensity granuar flow. Phys. Rev. E, 54:445-456, 1996. 67

[BMPV01] A. Baldassarri, U. Marini Bettolo Marconi, A. Puglisi, and A. Vulpiani. Driven granular gases with gravity. Phys. Rev. E, 64:011301-1-13,2001. 3

[BMSS02] J. Bougie, Sung Joon Moon, J.B. Swift, and Harry L. Swinney. Shocks in vertically oscillated granular layers. Phys. Rev. E, 66:051301, 2002. 2

[BP01] N. V. Brilliantov and T. Pöschel. Granular gases with impact-velocity dependent restitution coefficient. In T. Pöschel and S. Luding, editors, Granular Gases, pages 101-124, Berlin, 2001. Springer. 10

[BRM03] J. Javier Brey and M. J. Ruiz-Montero. Velocity distribution of fluidized granular gases in the presence of gravity. Phys. Rev. E, 67:021307, 2003. 4, 80

[BRMM00] J. Javier Brey, M. J. Ruiz-Montero, and F. Moreno. Boundary conditions and normal state for a vibrated granular fluid. Phys. Rev. E, 62:5339-5346, 2000. 2, 3, 42, 61,76 
[BSHP96] N. V. Brilliantov, F. Spahn, J. M. Hertzsch, and T. Pöschel. Model for collisions in granular gases. Phys. Rev. E, 53(5):5382, 1996. 11

[BSS99] C. Bizon, M. D. Shattuck, and J. B. Swift. Linear stability analysis of a vertically oscillated granular layer. Phys. Rev. E, 60:7210-7216, 1999. 8

[BT02a] A. Barrat and E. Trizac. Lack of energy equipartition in homogeneous heated binary granular mixtures. Granular Matter, 4(2):57-63, 2002. 4

[BT02b] A. Barrat and E. Trizac. Molecular dynamics simulations of vibrated granular gases. Phys. Rev. E, 66:051303, 2002. 2, 3, 4, 42, 63, 76, 80

[BT03] A. Barrat and E. Trizac. Random inelasticity and velocity fluctuations in a driven granular gas. Eur. Phys. J. E, 11(1):99-104, 2003. 81

[BTF01] A. Barrat, E. Trizac, and J. N. Fuchs. Heated granular fluids: the random restitution coefficient approach. Eur. Phys. J. E, 5(2):161-170, 2001. 2, 3

[CC70] S. Chapman and T. G. Cowling. The Mathematical Theory of Nonuniform Gases. Cambridge University Press, London, 1970. 67

[CGM95] P. Constantin, E. L. Grossman, and M. Mungan. Inelastic collisions of three particles on a line as a two-dimensional billiard. Physica D, 83:409, 1995. 29

[CLH00] R. Cafiero, S. Luding, and H. J. Herrmann. Two-dimensional granular gas of inelastic spheres with multiplicative driving. Phys. Rev. Lett., 84:6014-6017, 2000. 2, 3, 8, 86

[CLH02] R. Cafiero, S. Luding, and H. J. Herrmann. Rotationally driven gas of inelastic rough spheres. Europhys. Lett., 60(6):854-860, 2002. 8, 86

[CS69] W. F. Carnahan and K. E. Starling. Equation of state for nonattracting rigid spheres. J. Chem. Phys., 51(2):635-636, 1969. 17, 130

[DBS97] J. W. Dufty, J. J. Brey, and A. Santos. Kinetic models for hard sphere dynamics. Physica A, 240:212-220, 1997. 67

[DLK95] Y. Du, H. Li, and L. P. Kadanoff. Breakdown of hydrodynamics in a one-dimensional system of inelastic particles. Phys. Rev. Lett., 74(8):1268-1271, 1995. 1

[DP98] B. Drossel and T. Prellberg. Dynamics of a single particle in a horizontally shaken box. Eur. Phys. J. B, 1:533-543, 1998. 3

[Duf01] J. W. Dufty. Kinetic theory and hydrodynamics for rapid granular flow: A perspective. e-print cond-mat/0108444, 2001. 2

[EFL00] S. Fauve E. Falcon and C. Laroche. Experimental study of a granular gas fluidized by vibrations. In T. Pöschel and S. Luding, editors, Granular Gases, Lecture Notes in Physics 564, pages 244-253. Springer, Berlin, 2000. 2

[EP97] S. E. Esipov and T. Pöschel. The granular phase diagram. J. Stat. Phys., 86:13851395, 1997. 29 
[FFL99] E. Falcon, S. Fauve, and C. Laroche. Cluster formation, pressure and density measurements in a granular medium fluidized by vibrations. Eur. Phys. J. B, 9:183-186, 1999. 2

[FLCA94] S. F. Foerster, M. Y. Louge, H. Chang, and K. Allia. Measurements of the collision properties of small spheres. Phys. Fluids, 6(3):1108-1115, 1994. 9, 11

[FWE ${ }^{+99]}$ E. Falcon, R. Wunenburger, P. Evesque, S. Fauve, C. Chabot, Y. Garrabos, and D. Beysens. Cluster formation in a granular medium fluidized by vibrations in low gravity. Phys. Rev. Lett., 83:440-443, 1999. 2

[GG01] B. J. Glasser and I. Goldhirsch. Scale dependence, correlations, and fluctuations of stresses in rapid granular flows. Phys. Fluids, 13(2):407-420, 2001. 21, 23, 31

[God03] J. D. Goddard. Material instability in complex fluids. Annu. Rev. Fluid Mech., 35:113-133, 2003. 2

[Go198] I. Goldhirsch. Kinetics and dynamics of rapid granular flows. In H. J. Herrmann, J.-P. Hovi, and S. Luding, editors, Physics of dry granular media - NATO ASI Series E 350, pages 371 - 400, Dordrecht, 1998. Kluwer Academic Publishers. 31

[Gol00] I. Goldhirsch. Probing the boundaries of hydrodynamics. In T. Pöschel and S. Luding, editors, Granular Gases, Lecture Notes in Physics 564, pages 79-99. Springer, Berlin, 2000. 1

[Gol03] I. Goldhirsch. Rapid granular flows. Annu. Rev. Fluid Mech., 35:267-293, 2003. 1, $2,28,29$

[Gol04] I. Goldhirsch. Weakly frictional granular gases. In D. E. Wolf and M. Schreckenberg, editors, Traffic and Granular Flow, Singapore, 2004. World Scientific. 92

[GR65] I. S. Gradshteyn and I. M. Ryzhik. Table of Integrals, Series, and Products. Scripta Technica, New York, 1965. 124

[GS95] A. Goldshtein and M. Shapiro. Mechanics of collisional motion of granular materials. Part 1. General hydrodynamic equations. J. Fluid Mech., 282:75-114, 1995. 1, 63

[GS96] I. Goldhirsch and N. Sela. Origin of normal stress differences in rapid granular flows. Phys. Rev. E, 54(4):4458, 1996. 2

[GSB ${ }^{+98]}$ D. Goldman, M. D. Shattuck, C. Bizon, W. D. McCormick, J. B. Swift, and H. L. Swinney. Absence of inelastic collapse in a realistic three ball model. Phys. Rev. E, 57(4):R4831-R4833, 1998. 29

[GTZ93] I. Goldhirsch, M.-L. Tan, and G. Zanetti. A molecular dynamical study of granular fluids I: The unforced granular gas in two dimensions. Journal of Scientific Computing, 8:1-40, 1993. 2

[GZ93] I. Goldhirsch and G. Zanetti. Clustering instability in dissipative gases. Phys. Rev. Lett., 70(11):1619-1622, 1993. 2, 8 
[GZ96] G. Giese and A. Zippelius. Collision properties of one-dimensional granular particles with internal degrees of freedom. Phys. Rev. E, 54:4828, 1996. 29

[GZ99] F. Gerl and A. Zippelius. Coefficient of restitution for elastic disks. Phys. Rev. E, 59:2361-2372, 1999. 10

[GZBN97] E. L. Grossman, T. Zhou, and E. Ben-Naim. Towards granular hydrodynamics in two-dimensions. Phys. Rev. E, 55:4200, 1997. 1, 2, 3, 13, 42, 59, 60, 61, 63, 78

[Haf83] P. K. Haff. Grain flow as a fluid-mechanical phenomenon. J. Fluid Mech., 134:401430, 1983. 136

[HAZ99] M. Huthmann, T. Aspelmeier, and A. Zippelius. Granular cooling of hard needles. Phys. Rev. E, 60:654-659, 1999. 2

$\left[\mathrm{HCZ}^{+} 05\right]$ O. Herbst, R. Cafiero, A. Zippelius, H. J. Herrmann, and S. Luding. A driven twodimensional granular gas with coulomb friction. e-print cond-mat/0501223, 2005. 3 , $83,120,153$

[Hen75] D. Henderson. A simple equation of state for hard discs. Molec. Phys., 30(3):971972, 1975. 17, 49, 63, 68, 86

[Hen77] D. Henderson. Monte Carlo and perturbation theory studies of the equation of state of the two-dimensional lennard-jones fluid. Molec. Phys., 34(2):301-315, 1977. 86

[Her00] O. Herbst. Das Abkühlverhalten eines granularen Gases mit Coulomb'scher Reibung. Master's thesis, Georg-August-Universität Göttingen, 2000. (Diplomarbeit, German). Downloadable at http://www.theorie.physik.unigoettingen.de/ herbst/download/diplom.pdf. 11, 130

[HHL98] H. J. Herrmann, J.-P. Hovi, and S. Luding, editors. Physics of dry granular media NATO ASI Series E 350, Dordrecht, 1998. Kluwer Academic Publishers. 8

[HHZ00] O. Herbst, M. Huthmann, and A. Zippelius. Dynamics of inelastically colliding spheres with Coulomb friction: Relaxation of translational and rotational energy. Granular Matter, 2(4):211-219, 2000. 2, 9, 11, 29, 91, 92, 94, 101, 130, 136, 137, 153

[HM86] J. P. Hansen and I. R. McDonald. Theory of simple liquids. Academic Press Limited, London, 1986. 17, 40, 63

[HMOZ04] O. Herbst, P. Müller, M. Otto, and A. Zippelius. Local equation of state and velocity distributions of a driven granular gas. Phys. Rev. E, 70:051313-1-14, 2004. 3, 11, 153

[HMZ04] O. Herbst, P. Müller, and A. Zippelius. Local heat flux and energy loss in a 2d vibrated granular gas. e-print cond-mat/0412334, 2004. 153

[HOB00] M. Huthmann, J. A. G. Orza, and R. Brito. Dynamics of deviations from the gaussian state in a freely cooling homogeneous system of smooth inelastic particles. Granular Matter, 2(4):189-199, 2000. 2 
[HZ97] M. Huthmann and A. Zippelius. Dynamics of inelastically colliding rough spheres: Relaxation of translational and rotational energy. Phys. Rev. E, 56(6):6275-6278, 1997. $9,10,19,85,86,90,128$

[JNB96] H. M. Jaeger, S. R. Nagel, and R. P. Behringer. Granular solids, liquids, and gases. Rev. Mod. Phys., 68(4):1259-1273, 1996. 1, 2

[JR85] J. T. Jenkins and M. W. Richman. Kinetic theory for plane shear flows of a dense gas of identical, rough, inelastic, circular disks. Phys. of Fluids, 28:3485-3494, 1985. 2 , $5,49,63,67,68,69,71,72,74,85,86$

[JS83] J. T. Jenkins and S. B. Savage. A theory for the rapid flow of identical, smooth, nearly elastic, spherical particles. J. Fluid Mech., 130:187-202, 1983. 2

[JZ02] J. T. Jenkins and C. Zhang. Kinetic theory for identical, frictional, nearly elastic spheres. Physics of Fluids, 14(3):1228-1235, 2002. 102

[Kad99] L. P. Kadanoff. Built upon sand: Theoretical ideas inspired by granular flows. Rev. Mod. Phys., 71(1):435-444, 1999. 1

[KBN01] P. L. Krapivsky and E. Ben-Naim. Nontrivial velocity distributions in inelastic gases. J. Phys. A: Math. Gen., 35:L147-L152, 2001. 2

[KM04] E. Khain and B. Meerson. Oscillatory instability in a driven granular gas. Europhys. Lett., 65:193-199, 2004. 41

[Kum98] V. Kumaran. Temperature of a granular material "fluidized" by external vibrations. Phys. Rev. E, 57(5):5660-5664, 1998. 3, 13

[Kum99a] V. Kumaran. Nonequilibrium stationary states of a particle in a gravitational field driven by a vibrating surface. Phys. Rev. Lett., 82(4):3248, 1999. 13

[Kum99b] V. Kumaran. Velocity distribution for a dilute vibrated granular material. Phys. Rev. E, 59(4):4188-4202, 1999. 3

[KWG97] A. Kudrolli, M. Wolpert, and J. P. Gollub. Cluster formation due to collisions in granular material. Phys. Rev. Lett., 78(7):1383-1386, 1997. 2

$\left[\mathrm{LCB}^{+}\right.$94] S. Luding, E. Clément, A. Blumen, J. Rajchenbach, and J. Duran. The onset of convection in molecular dynamics simulations of grains. Phys. Rev. E, 50:R1762, 1994. 4

[LCG99] W. Losert, D. G. W. Cooper, and J. P. Gollub. Propagating front in an excited granular layer. Phys. Rev. E, 59:5855, 1999. 2

[LCRD96] S. Luding, E. Clément, J. Rajchenbach, and J. Duran. Simulations of pattern formation in vibrated granular media. Europhys. Lett., 36(4):247-252, 1996. 4, 29

[LG03] S. Luding and A. Goldshtein. Collisional cooling with multi-particle interactions. Granular Matter, 5(3):159-163, 2003. 29

[LHB94] S. Luding, H. J. Herrmann, and A. Blumen. Scaling behavior of 2-dimensional arrays of beads under external vibrations. Phys. Rev. E, 50:3100, 1994. 3, 29 
[LHMZ98] S. Luding, M. Huthmann, S. McNamara, and A. Zippelius. Homogeneous cooling of rough dissipative particles: Theory and simulations. Phys. Rev. E, 58:3416-3425, 1998. $2,19,86,128$

[LM98] S. Luding and S. McNamara. How to handle the inelastic collapse of a dissipative hard-sphere gas with the TC model. Granular Matter, 1(3):113-128, 1998. 3, 29, 32

[LMS02a] E. Livne, B. Meerson, and P. V. Sasorov. Symmetry breaking and coarsening of clusters in a prototypical driven granular gas. Phys. Rev. E, 66:050301, 2002. 1, 31, 41

[LMS02b] E. Livne, B. Meerson, and P. V. Sasorov. Symmetry-breaking instability and strongly peaked periodic clustering states in a driven granular gas. Phys. Rev. E, 65:021302$1-6,2002.1,2,31,41,61$

[LS00] S. Luding and O. Strauß. The equation of state for almost elastic, smooth, polydisperse granular gases for arbitrary density. In T. Pöschel and S. Luding, editors, Granular Gases, Lecture Notes in Physics 564, pages 389-409. Springer, Berlin, 2000. 3

[LSM99] S. Luding, O. Strauß, and S. McNamara. Segregation of polydisperse granular media in the presence of a temperature gradient. In T. Rosato, editor, IUTAM Symposium on Segregation in Granular Flows, pages 297-304, Dordrecht, 1999. Kluwer Academic Publishers. 8

[Lub91] B. D. Lubachevsky. How to simulate billards and similar systems. J. Comp. Phys., 94(2):255, 1991. 3, 29, 83

[Lud95] S. Luding. Granular materials under vibration: Simulations of rotating spheres. Phys. Rev. E, 52(4):4442, 1995. 4

[Lud01] S. Luding. Global equation of state of two-dimensional hard sphere systems. Phys. Rev. E, 63:042201-1-4, 2001. 2, 62, 64

[Lud03] S. Luding. Driven granular gases. In T. Pöschel and N. V. Brilliantov, editors, Granular Gas Dynamics, pages 293-316, Berlin, Germany, 2003. Springer. 86

[MB97] S. McNamara and J.-L. Barrat. Energy flux into a fluidized granular medium at a vibrating wall. Phys. Rev. E, 55:7767-7770, 1997. 3, 13

[MBD94] R. Mazighi, B. Bernu, and F. Delyon. Steady state of a column of shaked inelastic spheres. Phys. Rev. E, 50:4551, 1994. 29

[MGSB99] J. M. Montanero, V. Garzo, A. Santos, and J. J. Brey. Kinetic theory of simple granular shear flows of smooth hard spheres. J. Fluid Mech., 389:391-411, 1999. 2

[MPSS04] B. Meerson, T. Pöschel, P.V. Sasorov, and T. Schwager. Giant fluctuations at a granular phase separation threshold. Phys. Rev. E, 69:021302-1-9, 2004. 1, 2

[MS96] J. M. Montanero and A. Santos. Monte carlo simulation method for the Enskog equation. Phys. Rev. E, 54:438-444, 1996. 3 
[MSS01] S. J. Moon, M. D. Shattuck, and J. B. Swift. Velocity distributions and correlations in homogeneously heated granular media. Phys. Rev. E, 64:031303, 2001. 81

[MSS04] S. J. Moon, J. B. Swift, and H. L. Swinney. Steady state velocity distributions of an oscillated granular gas. Phys. Rev. E, 69:011301, 2004. 78

[MY92] S. McNamara and W. R. Young. Inelastic collapse and clumping in a one-dimensional granular medium. Phys. Fluids A, 4(3):496-594, 1992. 29

[MY94] S. McNamara and W. R. Young. Inelastic collapse in two dimensions. Phys. Rev. E, 50(1):R28-R31, 1994. 29

[OU98] J. S. Olafsen and J. S. Urbach. Clustering, order and collapse in a driven granular monolayer. Phys. Rev. Lett., 81:4369, 1998. 2, 8

[OU99] J. S. Olafsen and J. S. Urbach. Velocity distributions and density fluctuations in a 2d granular gas. Phys. Rev. E, 60:R2468, 1999. 8

[PCMP03] D. Paolotti, C. Cattuto, U. Marini Bettolo Marconi, and A. Puglisi. Dynamical properties of vibrofluidized granular mixtures. Granular Matter, 5(2):75-83, 2003. 4

[PLMV98] A. Puglisi, V. Loreto, U. M. B. Marconi, and A. Vulpiani. Clustering and nongaussian behavior in granular matter. Phys. Rev. Lett., 81:3848, 1998. 8

[PLMV99] A. Puglisi, V. Loreto, U. M. B. Marconi, and A. Vulpiani. Kinetic approach to granular gases. Phys. Rev. E, 59:5582-5595, 1999. 3, 4

[Rap88] D. C. Rapaport. Large-scale molecular dynamics simulation using vector and parallel computers. Computer Physics Reports, 9:1-54, 1988. 3, 29

[RBSS02] E. Rericha, C. Bizon, M. D. Shattuck, and H.L. Swinney. Shocks in supersonic sand. Phys. Rev. Lett., 88:014302, 2002. 2

[RC02] D. Risso and P. Cordero. Dynamics of rarefied granular gases. Phys. Rev. E, 65:021304-1-9, 2002. 3

[RM00] F. Rouyer and N. Menon. Velocity fluctuations in a homogeneous $2 d$ granular gas in steady state. Phys. Rev. Lett., 85(17):3676, 2000. 2, 4, 81

[RPBS99] Rosa Ramirez, Thorsten Pöschel, Nikolai V. Brilliantov, and Thomas Schwager. Coefficient of restitution of colliding viscoelastic spheres. Phys. Rev. E, 60:4465-4472, 1999. 10

[RRC00] R. Ramírez, D. Risso, and P. Cordero. Thermal convection in fluidized granular systems. Physical Review Letters, 85(6):1230-1233, 2000. 4

[RY84] F. J. Rogers and D. A. Young. New, thermodynamically consistent, integral equation for simple fluids. Phys. Rev. A, 30:999-1007, 1984. 40

[SG98] N. Sela and I. Goldhirsch. Hydrodynamic equations for rapid flows of smooth inelastic spheres to Burnett order. J. Fluid Mech., 361:41-74, 1998. 1, 2, 67 
[SGN96] N. Sela, I. Goldhirsch, and S. H. Noskowicz. Kinetic theoretical study of a simply sheared two-dimensional granular gas to Burnett order. Phys. Fluids, 8(9):2337, 1996. 2

[SK99] P. Sunthar and V. Kumaran. Temperature scaling in a dense vibrofluidized granular material. Phys. Rev. E, 60(5):1951-1956, 1999. 86

[SM01] R. Soto and M. Mareschal. Statistical mechanics of fluidized granular media: short range velocity correlations. Phys. Rev. E, 63:041303, 2001. 8, 17, 63

[SMR99] R. Soto, M. Mareschal, and D. Risso. Departure from Fourier's law for fluidized granular media. Phys. Rev Lett., 83(24):5003-5006, 1999. 8, 72

[Sun03] Sankaran Sundaresan. Instabilities in fluidized beds. Annu. Rev. Fluid Mech., 35:63$88,2003.2$

[TG98] M.-L. Tan and I. Goldhirsch. Rapid granular flows as mesoscopic systems. Phys. Rev. Lett., 81(14):3022-3025, 1998. 1

[Tos04] Giuseppe Toscani. Kinetic and hydrodynamic models of nearly elastic granular flows. Monatshefte Mathematik, 142:179 - 192, 2004. 1

[TV02] J. Talbot and P. Viot. Wall enhanced convection in vibrofluidized granular systems. Phys. Rev. Lett., 89(6):064301-1, 2002. 10

[UMS96] P. B. Umbanhowar, F. Melo, and H. L. Swinney. Localized excitations in a vertically vibrated granular layer. Nature, 382:793-796, 1996. 2

[UMS98] P. B. Umbanhowar, Francisco Melo, and Harry L. Swinney. Periodic, aperiodic, and transient patterns in vibrated granular layers. Physica A, 249:1-9, 1998. 2

[UO01] Jeffrey S. Urbach and Jeffrey S. Olafsen. Experimental observations of nonequilibrium distributions and transitions in a $2 \mathrm{~d}$ granular gas. In Thorsten Pöschel and Stefan Luding, editors, Granular Gases, Lecture Notes in Physics 564, pages $410-428$, Berlin, 2001. Springer. 2

[US00] P. B. Umbanhowar and H. L. Swinney. Wavelength scaling and square/stripe and grain mobility transitions in vertically oscillated granular layers. Physica A, 288:344$362,2000.2$

[vdWvdMVL01] K. van der Weele, D. van der Meer, M. Versluis, and D. Lohse. Hysteretic custering in granular gas. Europhys. Lett., 53(3):328-334, 2001. 2

[VL82] L. Verlet and D. Levesque. Integral equations for classical fluids. III. the hard disks system. Mol. Phys., 46(5):969-980, 1982. 86

[vNE98] T. P. C. van Noije and M. H. Ernst. Velocity distributions in homogeneous granular fluids: the free and the heated case. Granular Matter, 1(2):57-64, 1998. 2, 3, 8, 17, 81

[vNEB98] T. P. C. van Noije, M. H. Ernst, and R. Brito. Ring kinetic theory for an idealized granular gas. Physica A, 251:266-283, 1998. 2 
[vNETP99] T. P. C. van Noije, M. H. Ernst, E. Trizac, and I. Pagonabarraga. Randomly driven granular fluids: Large scale structure. Phys. Rev. E, 59:4326-4341, 1999. 3, 8

[VT04] P. Viot and J. Talbot. Thermalization of an anisotropic granular particle. Phys. Rev. E, 69:051106, 2004. 2

[vZKG $\left.{ }^{+} 04\right]$ J. S. van Zon, J. Kreft, Daniel I. Goldman, D. Miracle, J.B. Swift, and Harry L. Swinney. Crucial role of sidewalls in velocity distributions in quasi-two-dimensional granular gases. Phys. Rev. E, 70:040301-1-4, 2004. 5

[vZM04] J. S. van Zon and F. C. MacKintosh. Velocity distributions in dissipative granular gases. Phys. Rev. Lett., 93(3):038001-1-4, 2004. 4, 80, 81

[WB86] O. R. Walton and R. L. Braun. Viscosity, granular-temperature, and stress calculations for shearing assemblies of inelastic, frictional disks. J. Rheol., 30(5):949-980, 1986. $1,5,9,11,85,101$

[WHJ95] S. Warr, J. M. Huntley, and G. T. H. Jacques. Fluidization of a two-dimensional granular system: Experimental study and scaling behavior. Phys. Rev. E, 52(5):55835595, 1995. 2

[WHP01] R. D. Wildman, J. M. Huntley, and D. J. Parkar. Convection in highly fluidized threedimensional granular beds. Phys. Rev. Lett., 86(15):3304-3307, 2001. 10

[WM96] D. R. M. Williams and F. C. MacKintosh. Driven granular media in one dimension: Correlations and equation of state. Phys. Rev. E, 54(1):R9-R12, 1996. 3

[WP02] R. D. Wildman and D. J. Parkar. Energy nonequipartition in a vibrated granular gas mixture. Phys. Rev. Lett., 88(19):064301, 2002. 10 


\section{Note on the Bibliography}

At the end of each entry in the bibliography the reader may find the page numbers (in this thesis) where the corresponding paper has been cited. Of course, these numbers do not belong to the cited articles themselves. 



\section{Acknowledgements}

One accomplishes little alone. As a physicist I strongly rely on the society; and I am thankful to live in a politically and economically stable country with freedom and democracy. Many people have helped to make this thesis possible. Only a few can be mentioned here to whom I am extraordinarily grateful. Thank you

- Prof. Annette Zippelius for motivating and guiding my work, for never-ending support and assistance, for encouraging me to due diligence, for reliable advice, and for very fruitful discussions

- PD Dr. Peter Müller for support and assistance, for willingly discussing every physics problem, and of course for the nice and fruitful collaboration on vibrated systems

- Dr. Matthias Otto for support and discussions

- Dr. Timo Aspelmeier and Dr. Martin Huthmann for discussions and providing the first version of the simulation developed

- Prof. Stefan Luding for the very enjoyable and fruitful cooperation on frictional particles and for invitations to Stuttgart, Delft, and Leiden

- Prof. Isaac Goldhirsch for inspiring discussions in Leiden and for suggesting to measure the heat flux in vibrated systems

- Dr. Bernd Burghardt for giving me an introduction to Perl which simplified my life considerably as well as for many lunch sessions at the Klinikum

- Dr. Hans Vollmayr for pointing out the importance of the elastic limit in vibrated systems

- Dr. Jürgen Holm for maintaining the computer system necessary for the simulations

- Dipl. Phys. Stephan Kramer for many breakfast sessions, private discussions - not only in our shared office, and for maintaining condor without which the simulations would have hardly been possible

- Dr. Henning Löwe and Dr. Hergen Schulze for the enjoyable times in our shared office(s) in the Villa

- all present and former members of the Institut für Theoretische Physik Göttingen, especially the Villa group, for the enjoyable working atmosphere

- Giesela Mesecke, Katrin Glormann, and Gabriele Schubert for an open ear at all times

- Christine Menzel for the time together, many fruitful discussions, and proof-reading

- Gunda Linke for your support and everything you have done for me

- Emmi Leijala, Michael Schäfer, and Jens-Arne Reumschüssel for your friendship

- my family, especially my parents, for support in good and bad times. 



\section{List of Publications and Meetings}

\section{Publications}

O. Herbst, M. Huthmann, and A. Zippelius, Dynamics of inelastically colliding spheres with Coulomb friction: Relaxation of translational and rotational energy, Granular Matter 2(4), 211 - 219 (2000). (Ref. [HHZ00])

O. Herbst, P. Müller, M. Otto, and A. Zippelius, Local Equation of State and Velocity Distributions of a Driven Granular Gas, Phys. Rev. E 70, 051313-1 - 14 (2004). (Ref. [HMOZ04])

O. Herbst, P. Müller, and A. Zippelius, Local heat flux and energy loss in a $2 D$ vibrated granular gas, e-print cond-mat/0412334. (Ref. [HMZ04])

O. Herbst, R. Cafiero, A. Zippelius, H. J. Herrmann, and S. Luding, A driven two-dimensional granular gas with Coulomb friction, e-print cond-mat/0501223. (Ref. $\left[\mathrm{HCZ}^{+} 05\right]$ )

\section{Meetings}

1999 Granular Gases, Bad Honnef, Germany

2000 Complex Fluids summer school, Bayreuth, Germany

2001 Computational Physics, Bad Honnef, Germany

2003 MECO 28 Statistical Physics, Saarbrücken, Germany (poster)

2003 DPG Frühjahrstagung, Dresden, Germany (talk, poster)

2003 From Granular to Nano Materials, Leiden, The Netherlands (invited talk)

2003 Southern Workshop on Granular Materials, Pucón, Chile (talk, poster)

2005 Kavli Granular Physics, Santa Barbara, California, U.S.A. (invited) 



\section{Curriculum Vitae}

Olaf Herbst

Leonard-Nelson-Str. 32

37073 Göttingen

E-Mail: olaf.herbst@gmx.net

Homepage: www.herbstland.de

\section{Persönliche Daten}

Geboren am 24. Dezember 1973 in Hamburg

Staatsangehörigkeit: deutsch

\section{Bildungsweg}

\begin{tabular}{|c|c|}
\hline $1980-1984$ & Grundschule Marmstorf, Hamburg-Harburg \\
\hline $1984-1993$ & Friedrich-Ebert-Gymnasium, Hamburg-Harburg \\
\hline $1990-1991$ & Cloquet High School, Cloquet, Minnesota, U.S.A. \\
\hline 1993 & Abitur \\
\hline $1993-1997$ & Physik- und Mathematikstudium, Georg-August-Universität Göttingen \\
\hline Okt. 1996 & Vordiplom Physik \\
\hline Feb. 1997 & Vordiplom Mathematik \\
\hline $1997-1998$ & Physikstudium, University of California Santa Barbara, Kalifornien, U.S.A. \\
\hline $1998-2000$ & Physikstudium, Georg-August-Universität Göttingen \\
\hline 2000 & Diplom Physik \\
\hline $2000-2005$ & Promotionsstudium Physik, Georg-August-Universität Göttingen \\
\hline Jan. 2005 & Vorlage der Dissertation \\
\hline
\end{tabular}

Göttingen, 20. Januar 2005 